\title{
The Effects of Pharmaceutical \\ Excipients on Drug Disposition
}

Talia Rae Buggins

A thesis submitted to Cardiff University in accordance with the regulations for the degree of Doctor of Philosophy

Welsh School of Pharmacy

Cardiff University

April 2007 
All rights reserved

\section{INFORMATION TO ALL USERS}

The quality of this reproduction is dependent upon the quality of the copy submitted.

In the unlikely event that the author did not send a complete manuscript and there are missing pages, these will be noted. Also, if material had to be removed, a note will indicate the deletion.

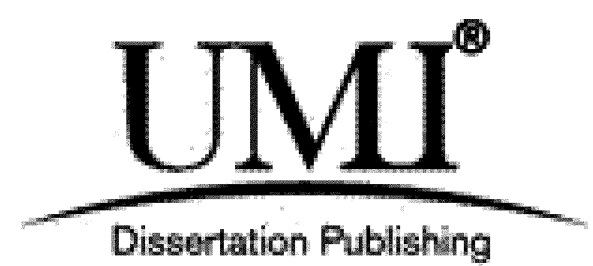

UMI U584177

Published by ProQuest LLC 2013. Copyright in the Dissertation held by the Author.

Microform Edition (c) ProQuest LLC.

All rights reserved. This work is protected against unauthorized copying under Title 17, United States Code.

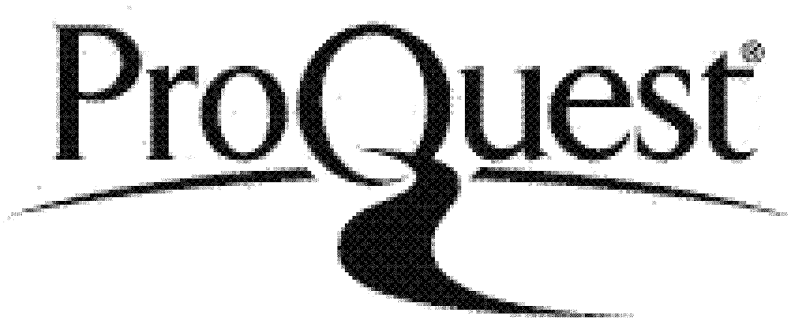

ProQuest LLC

789 East Eisenhower Parkway

P.O. Box 1346

Ann Arbor, MI 48106-1346 


\section{Declaration}

\section{DECLARATION}

This work has not previously been accepted in substance for any degree and is not concurrently submitted in candidature for any degree.

Signed

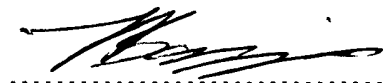
(candidate) Date $7 / 5 / 17$

\section{STATEMENT 1}

This thesis is being submitted in partial fulfillment of the requirements for the degree of $\mathrm{PhD}$.

Signed<smiles>Cc1ccc(C(C)C)c2c1CCCC2</smiles>
(candidate) Date .7/S./. 7.

\section{STATEMENT 2}

This thesis is the result of my own independent work/investigation, except where otherwise stated. Other sources are acknowledged by explicit references.

Signed

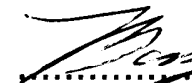

(candidate)

Date .7/SICG.

\section{STATEMENT 3}

I hereby give consent for my thesis, if accepted, to be available for photocopying and for inter-library loan, and for the title and summary to be made available to outside organisations.

Signed<smiles>CC1C2C=CC(C2)C1C</smiles>
(candidate)

Date $7 / 5 /$. 


\section{Acknowledgements}

I would like to thank my supervisor Glyn Taylor for his wisdom, patience and support throughout this project. I would also like to thank my industrial supervisors, Paul Dickinson and Marcel DeMatas, for their input and their enthusiasm for the project. I would like to thank Mark Gumbleton for allowing the use of his facilities for the cell experiments, and for giving me the benefit of his expertise in this area. I would also like to thank PCB, especially Mat, Chris and Danielle, for their support and the endless cups of tea. I would like to thank Mohammed Yasin for his help with the LC/MS/MS analysis. I would also like to thank the technical staff for their assistance. Thanks are also due to AstraZeneca and the Welsh School of Pharmacy for funding. Finally I would like to thank my husband and family, for supporting me in this and everything I do. 


\section{Summary}

This thesis investigates the potential of some commonly used pharmaceutical excipients to alter drug pharmacokinetics. In breath test studies, $3.2 \mathrm{ml} / \mathrm{kg}$ DMSO prolonged the half-life of ${ }^{14} \mathrm{C}$ aminopyrine, -erythromycin and -NDMA, indicating in vivo inhibition of metabolism by CYP3A (erythromycin), CYP2E1 (NDMA) and the variety of enzymes that metabolise aminopyrine (CYP 2C11, 2C12, 2B1 and 2B2). However, no effects were apparent at doses typically used in pre-clinical formulations. Aminopyrine and erythromycin breath tests were not affected by propylene glycol (PG) or Solutol $\mathrm{HS} 15$, however PG did significantly increase ${ }^{14} \mathrm{CO}_{2}$ exhalation halflife in the NDMA breath test, suggesting a specific effect on metabolism by CYP2E1. While two $2 \mathrm{ml} / \mathrm{kg}$ doses of DMSO increased plasma $\alpha_{1}$-AGP levels, none of the excipients tested consistently affected plasma $\alpha_{1}$-AGP at doses commonly used in pre-clinical formulations, suggesting that they are unlikely to increase protein binding by this mechanism. Transport studies in MDCK-MDR1 cells demonstrated an inhibitory effect of $0.1 \%$ Tween 80 and Solutol HS15 on P-Gp. In vivo, Tween inhibited the biliary elimination of ${ }^{99 \mathrm{~m}}$ Tc-MIBI but not ${ }^{99 \mathrm{~m}}$ Tc-HIDA, indicating inhibition of P-Gp, while Solutol inhibited the biliary elimination of both radiopharmaceuticals, suggesting inhibition of MRP and possibly PGp. Pre-treatment with $4 \mathrm{ml} / \mathrm{kg}$ DMSO substantially impaired the renal elimination of ${ }^{99 \mathrm{~m}}$ Tc-DTPA. In contrast, $20 \% 1.8 \mathrm{ml} / \mathrm{kg}$ DMSO significantly increased ${ }^{99 \mathrm{~m}}$ Tc-DTPA uptake into the kidneys, suggesting an increase in GFR. Both Tween and Solutol delayed ${ }^{99 m}$ Tc-DTPA elimination from the kidneys in some rats, without affecting GFR. However, Solutol did not significantly affect the pharmacokinetics of OAT or OCT substrates, suggesting it did not affect active renal secretion by these transporters. These results demonstrate that excipients can influence drug pharmacokinetics in vivo, after a single acute dose at levels commonly used in preclinical studies. 


\section{Abbreviations}

$\alpha$

$\alpha_{1}-\mathrm{AGP}$

$\beta$

${ }^{99 m}$ TC-DTPA

${ }^{99 m}$ TC-HIDA

${ }^{99 m}$ TC-MIBI

$A_{e}$

APBT

AUC

AUMC

CBT

$C D$

CER

CL

$\mathrm{CL}_{\text {int }}$

$\mathrm{C}_{\max }$

DMSO

EMBT

$F$

$f_{u}$

GFR

distribution rate constant

$\alpha_{1}$-acid glycoprotein

elimination rate constant

${ }^{99 m}$ Tc-Diethylenetriamine pentaacetic acid

${ }^{99 m} \mathrm{Tc}-\mathrm{N}-(2,6$-dimethylphenylcarbamoylmethyl)

iminodiacetic acid

${ }^{99 m}$ Tc-sesta-2-methoxy isobutile isonitrile

amount excreted unchanged in urine

aminopyrine breath test

area under the concentration vs. time curve

area under the first-moment vs. time curve

caffeine breath test

cyclodextrin

${ }^{14} \mathrm{CO}_{2}$-exhalation rate

clearance

intrinsic clearance

maximum plasma concentration

dimethyl sulfoxide

erythromycin breath test

bioavailable fraction

fraction unbound

glomerular filtration rate 


\begin{tabular}{|c|c|}
\hline$H P-\beta-C D$ & hydroxypropyl- $\beta$-cyclodextrin \\
\hline$H P-\gamma-C D$ & hydroxypropyl- $y$-cyclodextrin \\
\hline i.p. & intraperitoneal \\
\hline i.v. & intravenous \\
\hline$k_{1}$ & rate constant of first exponential decline \\
\hline MAT & mean absorption time \\
\hline$M e-\beta-C D$ & methyl- $\beta$-cyclodextrin \\
\hline MRT & mean residence time \\
\hline NDMA BT & nitrosodimethlyamine breath test \\
\hline PEG & polyethylene glycol \\
\hline PG & propylene glycol \\
\hline RBF & renal blood flow \\
\hline SBE- $\beta-C D$ & sulfobutylether- $\beta$-cyclodextrin \\
\hline$t_{1 / 2}$ & half-life \\
\hline TEER & trans-epithelial electrical resistance \\
\hline$t_{\max }$ & time of maximum plasma concentration \\
\hline v & volume of distribution \\
\hline$V_{s s}$ & steady-state volume of distribution \\
\hline CMC & critical micelle concentration \\
\hline CYP450 & cytochrome P450 \\
\hline $\mathrm{CL}_{\mathrm{H}}$ & hepatic clearance \\
\hline$C L_{R}$ & renal clearance \\
\hline DMA & dimethylacetamide \\
\hline DTPA & diethylene triamine pentaacetic acid \\
\hline$Q_{H}$ & hepatic blood flow \\
\hline
\end{tabular}




\section{Contents}

Declaration

Acknowledgements

Summary iv

Abbreviations $v$

$\begin{array}{ll}\text { Chapter 1: Literature review } & 1\end{array}$

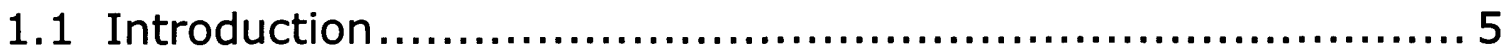

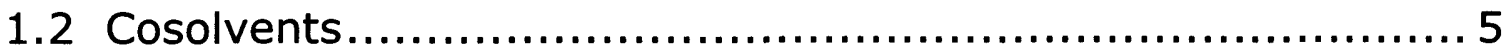

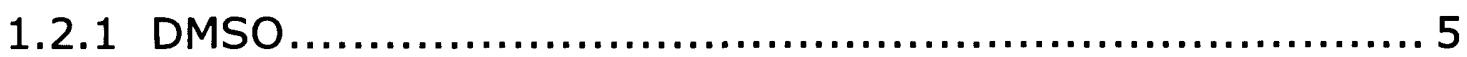

1.2.1.1 Effects of DMSO on metabolism ....................... 5

1.2.1.2 Effects of DMSO on renal elimination .................. 6

1.2.1.3 Effects of DMSO on oral absorption..................... 7

1.2.1.4 Effects of DMSO on distribution ....................... 7

1.2 .2 Ethanol....................................................... 9

1.2.2.1 Effects of ethanol on metabolism...................... 9

1.2.2.2 Effects of ethanol on hepatic blood flow ............... 11

1.2.2.3 Effects of ethanol on renal elimination ................ 12

1.2 .3 Propylene glycol........................................... 13

1.2.3.1 Effects of PG on metabolism ......................... 13

1.2.3.2 Effects of PG on distribution and renal excretion..... 14

1.2.3.3 Effects of PG on oral absorption ....................... 14

1.2.4 PEGs .......................................................... 15

1.2.4.1 Effects of PEGs on oral absorption ................... 15

1.2.4.2 Effects of PEGs on renal elimination................... 17

1.2.5 Other cosolvents ............................................ 18

1.2.5.1 Effects of other cosolvents on metabolism ............. 18

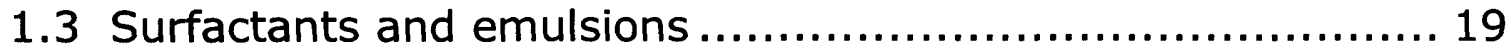

1.3.1 Cremophor.................................................. 19

1.3.1.1 Effects of Cremophor on pharmacokinetics ........... 20

1.3.1.2 Effects of Cremophor on distribution in blood ........ 22

1.3.1.3 Effects of Cremophor on hepatic elimination .......... 23

1.3.1.4 Effects of Cremophor on renal elimination ............. 24

1.3.1.5 Effects of Cremophor on P-Gp........................ 24

1.3.1.6 Effects of Cremophor on oral absorption............... 25

1.3.2 Tween .......................................................... 27

1.3.2.1 Effects of Tween on P-Gp ........................... 27

1.3.2.2 Effects of Tween on elimination...................... 28

1.3.2.3 Effects of Tween on distribution ....................... 30

1.3.2.4 Effects of Tween on pharmacokinetics after i.p. administration ................................................ 33

1.3.3 Solutol......................................................... 34 
1.3.3.1 Effects of Solutol on metabolism ..................... 34

1.3.3.2 Effects of Solutol on in vivo pharmacokinetics ........ 35

1.3.3.3 Effects of Solutol on plasma protein binding.......... 37

1.3.3.4 Effects of Solutol on oral absorption ................... 37

1.3.4 Other emulsion systems .................................... 38

1.3.4.1 Effects of other emulsion systems on

pharmacokinetics.............................................. 38

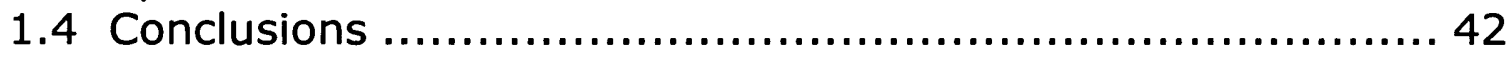

Chapter 2: Effects of excipients on in vivo metabolism 44

2.1 Introduction ...................................................... 46

2.2 Methods and Materials ......................................... 52

2.2.1 Materials....................................................... 52

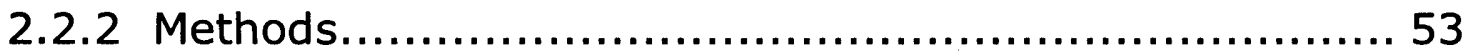

2.3 Results........................................................ 57

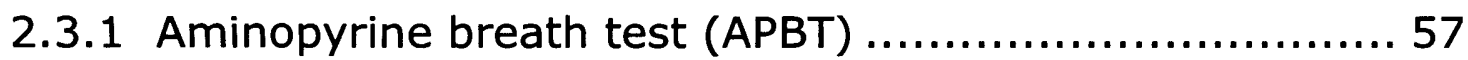

2.3.2 Erythromycin breath test (EMBT) .........................63 63

2.3.3 NDMA breath test (NDMA BT) ............................ 69

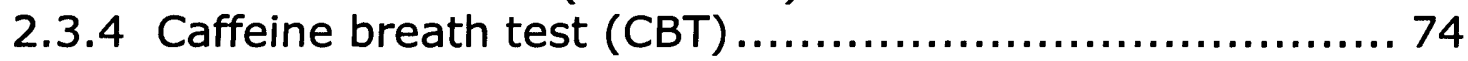

2.4 Discussion ....................................................... 76

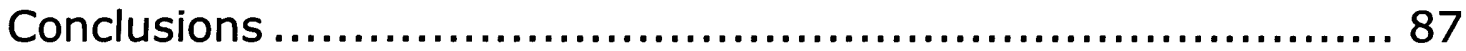

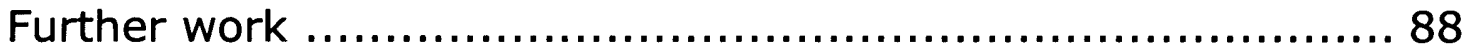

Chapter 3: Effects of excipients on $\alpha_{1}$-acid glycoprotein plasma $\begin{array}{ll}\text { levels in vivo } & 89\end{array}$

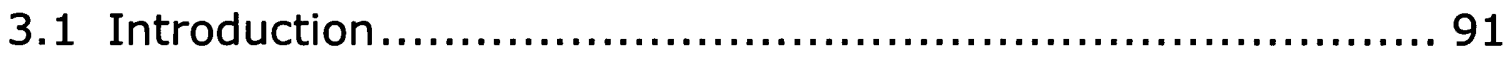

3.2 Methods and materials ......................................... 96

3.2.1 Materials......................................................... 96

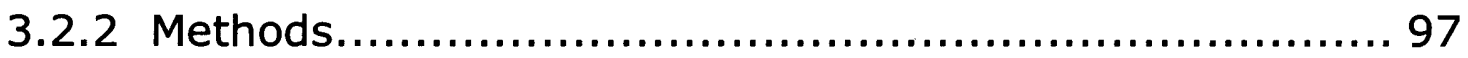

3.3 Results..................................................... 101

3.4 Discussion ...................................................... 106

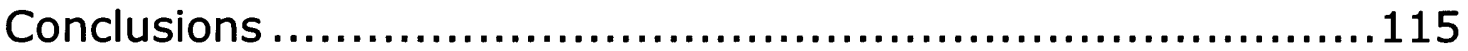

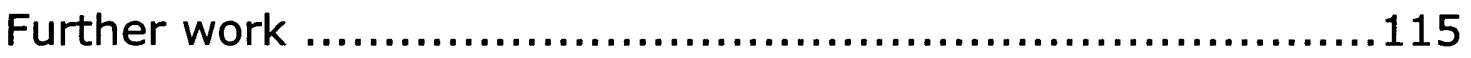

Chapter 4: Effects of excipients on P-Gp and ${ }^{99 m} T C-M I B I$ transport in vitro

4.1 Introduction................................................. 119

4.2 Materials and Methods......................................... 125

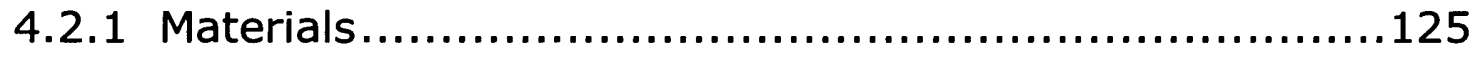

4.2.2 Methods....................................................... 
4.2.2.1 Cell culture .............................................126

4.2.2.2 Apical to basolateral $(A B)$ vinblastine and sucrose transport studies ............................................... 128 4.2.2.3 Bi-directional ${ }^{99 \mathrm{~m}} \mathrm{Tc}-\mathrm{MIBI}$, sucrose and vinblastine transport studies ..............................................129

4.2.2.4 Data analysis ........................................130

4.3 Results........................................................ 132

4.3.1 $\mathrm{AB}{ }^{3} \mathrm{H}$-vinblastine and ${ }^{14} \mathrm{C}$-sucrose transport .............132

4.3.2 Bi-directional ${ }^{3} \mathrm{H}$-vinblastine and ${ }^{14} \mathrm{C}$-sucrose transport .. 140

4.3.3 Bi-directional ${ }^{99 m}$ Tc-MIBI transport ........................143

4.3.4 Bi-directional ${ }^{99 \mathrm{~m}} \mathrm{Tc}$ transport .............................. 148

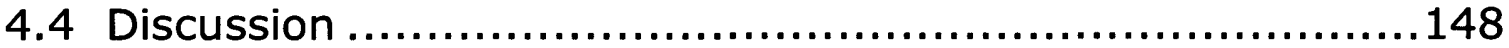

Conclusions ........................................................ 160

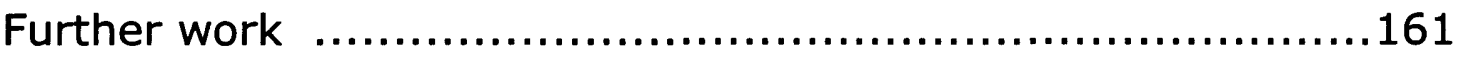

Chapter 5: In vivo disposition of ${ }^{99 m}$ TC-MIBI and ${ }^{99 m}$ TC-HIDA 163

1. Introduction ..................................................... 165

2. Methods and materials .......................................... 171

2.1 Materials ..................................................... 171

2.2 Methods ..................................................... 172

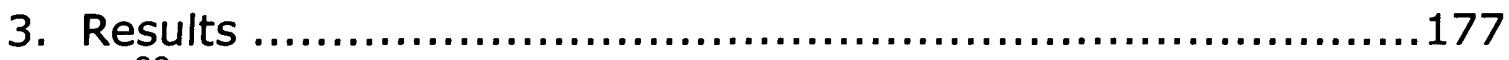

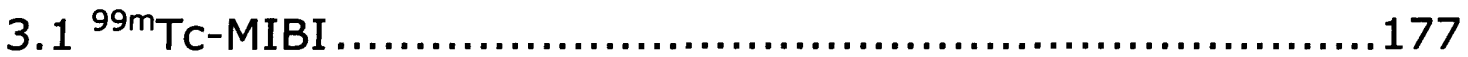

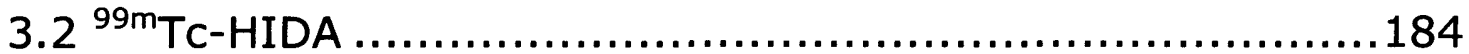

3.3 Effect of injection site retention ............................190

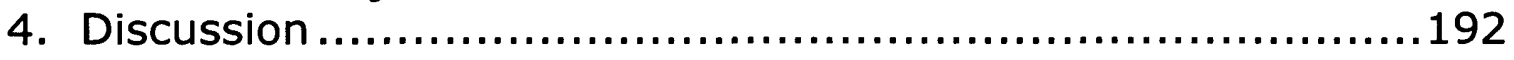

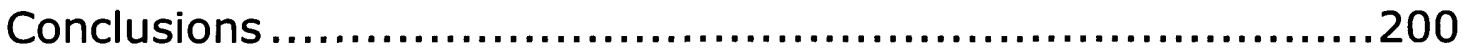

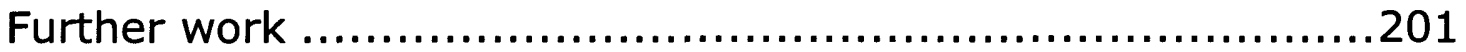

Chapter 6: Effects of excipients on renal elimination 202

6.1 Introduction.................................................204

6.2 Methods and materials ........................................ 211

6.2.1 Materials and equipment ................................... 211

Gamma scintigraphy .......................................211

6.2.2 Method development ..................................... 212

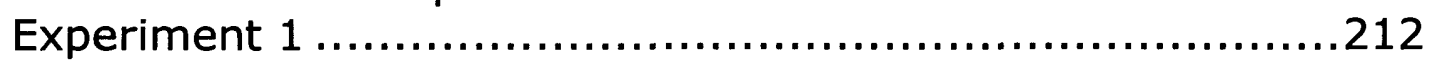

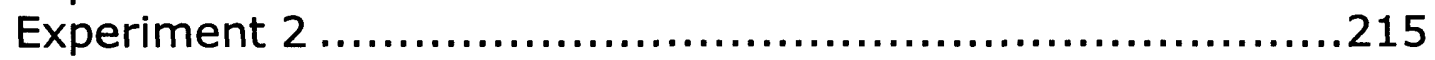

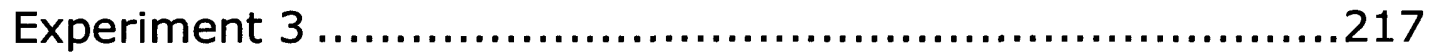

Positive control ................................................220

6.2.3 Crossover-type method ...................................224

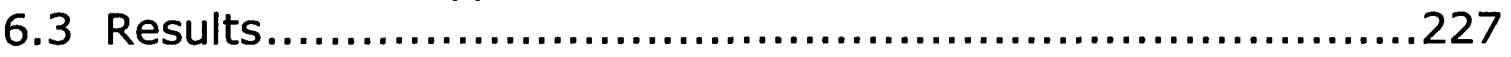

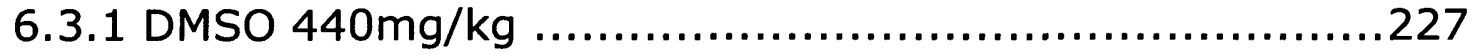

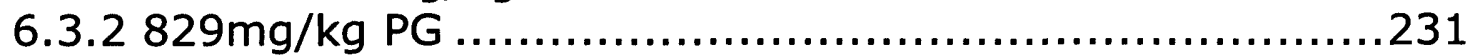

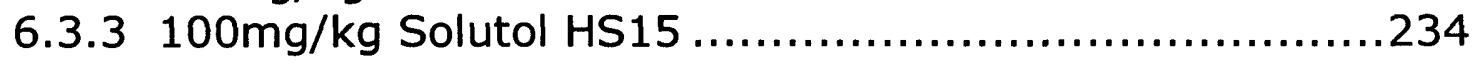




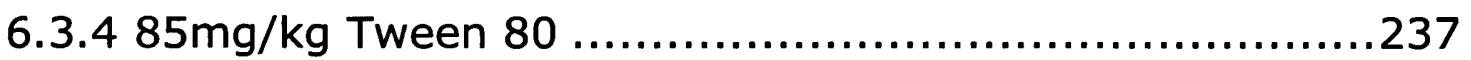

6.3.5 Positive control $-4400 \mathrm{mg} / \mathrm{kg}$ DMSO........................240

6.3.6 Comparison of data from control renograms ...............241

6.4 Discussion ....................................................242

Conclusions ...................................................... 255

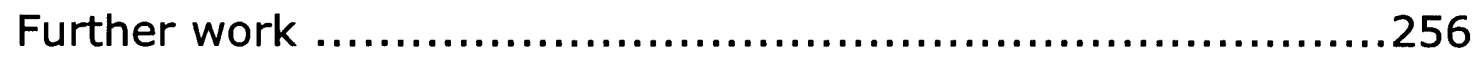

Chapter 7: Effects of excipients on the pharmacokinetics of benzylpenicillin and cimetidine 257

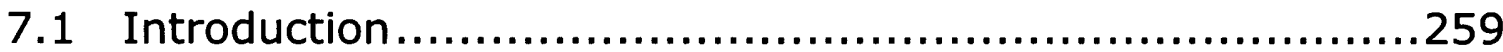

7.2 Methods and Materials........................................264

7.2.1 Materials........................................................264

7.2.2 Methods...................................................... 264

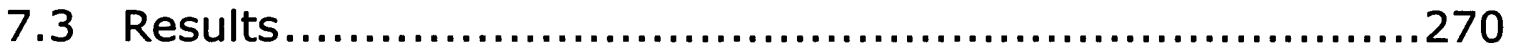

7.3.1 Benzylpenicillin ...........................................270

7.3.2 Cimetidine ................................................274

7.3.3 Effect of Solutol HS15 on LC/MS/MS analysis .............281

7.4 Discussion ....................................................281

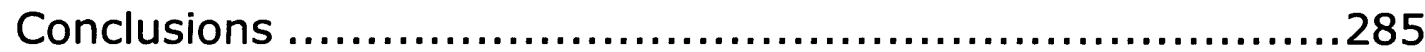

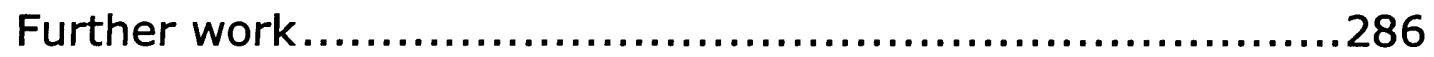

$\begin{array}{ll}\text { Chapter 8: Discussion } & 287\end{array}$

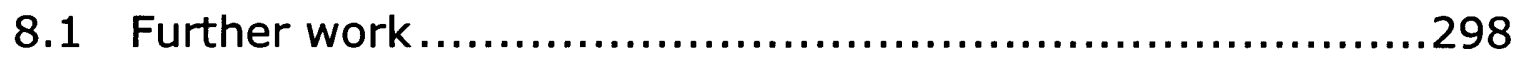

$\begin{array}{ll}\text { References } & 300\end{array}$

Appendix 1: Tables of reported excipient effects on pharmacokinetics

Table 1: Summary of reported effects of DMSO on pharmacokinetics

Table 2: Summary of reported effects of ethanol on

A2 pharmacokinetics............................................... A4

Table 3: Summary of reported effects of propylene glycol on pharmacokinetics................................................ A6

Table 4: Summary of reported effects of PEG on pharmacokinetics A7

Table 5: Summary of reported effects of DMA on pharmacokinetics

Table 6: Summary of reported effects of glycofurol on A8 pharmacokinetics A8 
Table 7: Summary of reported effects of cyclodextrins on pharmacokinetics.

Table 8: Summary of reported effects of Cremophor on pharmacokinetics

Table 9: Summary of reported effects of Tween on pharmacokinetics.

Table 10: Summary of reported effects of Solutol on pharmacokinetics.

Table 11: Summary of reported effects of various emulsion formulations on pharmacokinetics 


\section{Chapter 1:}

Literature Review 


\section{Contents}

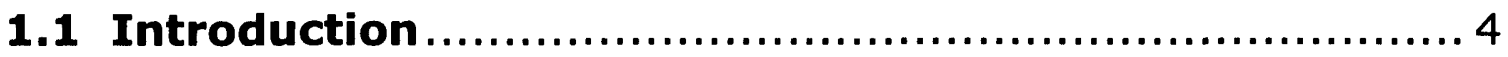

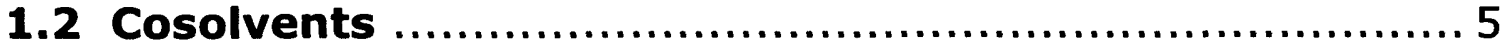

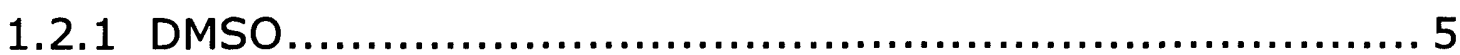

1.2.1.1 Effects of DMSO on metabolism .......................... 5

1.2.1.2 Effects of DMSO on renal elimination ..................... 6

1.2.1.3 Effects of DMSO on oral absorption........................ 7

1.2.1.4 Effects of DMSO on distribution............................ 7

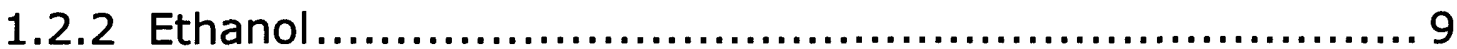

1.2.2.1 Effects of ethanol on metabolism ........................ 9

1.2.2.2 Effects of ethanol on hepatic blood flow ................ 11

1.2.2.3 Effects of ethanol on renal elimination .................. 12

1.2 .3 Propylene glycol................................................ 13

1.2.3.1 Effects of PG on metabolism ............................. 13

1.2.3.2 Effects of $P G$ on distribution and renal excretion..... 14

1.2.3.3 Effects of PG on oral absorption ...................... 14

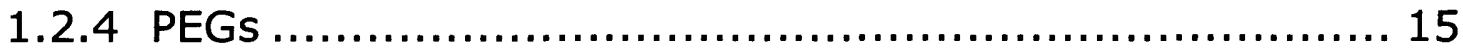

1.2.4.1 Effects of PEGs on oral absorption ................... 15

1.2.4.2 Effects of PEGs on renal elimination.................... 17

1.2 .5 Other cosolvents ................................................. 18

1.2.5.1 Effects of other cosolvents on metabolism ............. 18

1.3 Surfactants and emulsions .................................... 19

1.3.1 Cremophor......................................................... 19

1.3.1.1 Effects of Cremophor on pharmacokinetics ............ 20

1.3.1.2 Effects of Cremophor on distribution in blood ......... 22

1.3.1.3 Effects of Cremophor on hepatic elimination .......... 23

1.3.1.4 Effects of Cremophor on renal elimination ............. 24

1.3.1.5 Effects of Cremophor on P-Gp........................... 24

1.3.1.6 Effects of Cremophor on oral absorption................ 25

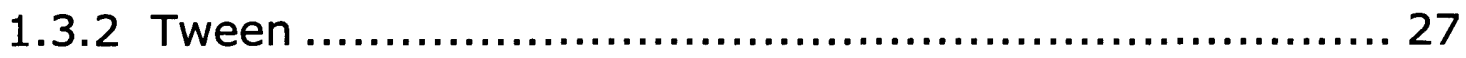

1.3.2.1 Effects of Tween on P-Gp ............................... 27 
1.3.2.2 Effects of Tween on elimination...................... 28

1.3.2.3 Effects of Tween on distribution ....................... 30

1.3.2.4 Effects of Tweeen on pharmacokinetics after i.p. administration ................................................. 33

1.3.3 Solutol........................................................ 34

1.3.3.1 Effects of Solutol on metabolism ..................... 34

1.3.3.2 Effects of Solutol on in vivo pharmacokinetics ....... 35

1.3.3.3 Effects of Solutol on plasma protein binding.......... 37

1.3.3.4 Effects of Solutol on oral absorption ................. 37

1.3.4 Other emulsion systems ................................... 38

1.3.4.1 Effects of other emulsion systems on

pharmacokinetics.............................................. 38

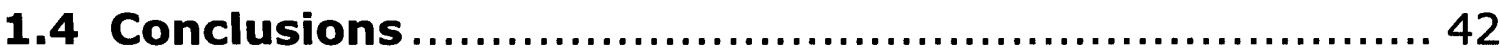




\subsection{Introduction}

The poor aqueous solubility of many new drug entities necessitates the use of various co-solvents and excipients to produce formulations suitable for i.v. dosing. There is evidence in the literature that such formulations have the ability to affect the pharmacokinetics of the drug, through a variety of effects on distribution and elimination. In early pharmacokinetic studies, designed to determine the intrinsic pharmacokinetic characteristics of a drug, it is desirable to avoid any alteration of drug pharmacokinetics by formulation additives (Bittner and Mountfield, 2002). Renewed interest in this field was sparked when the profound effect of Cremophor EL on pharmacokinetics of paclitaxel became apparent (Sparreboom et al., 1996; van Tellingen et al., 2000); however, the potential of excipients to affect drug pharmacokinetics has been reported as early as the 1960s (Brink and Stein, 1967). The potential impact of various formulation strategies used in drug discovery on pharmacokinetics were reviewed by Bittner and Mountfield (2002). This chapter will review some of the reported effects of commonly used pharmaceutical excipients on drug disposition. The focus will be on excipients likely to be used in parenteral formulations for pre-clinical studies. 
Due to the large amount of literature on this subject, it will not be possible to cover it all here. Therefore this chapter will focus on studies which demonstrate the potential of the excipients to affect pharmacokinetics. Appendix 1 contains tables describing the reported effects of various excipients, including some studies which have not been discussed in this chapter. There is also a table detailing reported effects of cyclodextrins on drug pharmacokinetics, which have not been included in this chapter due to space limitations.

\subsection{Cosolvents}

\subsubsection{DMSO}

\subsubsection{Effects of DMSO on metabolism}

The effects of DMSO on metabolism have been extensively investigated. It has been shown to inhibit CYP1A2, 2C8/9, 2C19, 2D6, $2 \mathrm{E} 1$ and 3A4, and arylhydrocarbon

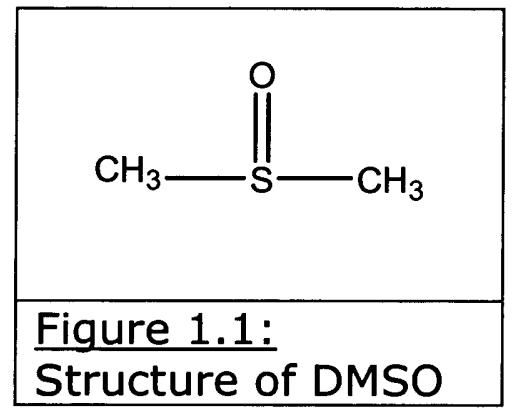
hydroxylase, in liver microsomes (Yoo et al., 1987; Chauret et al., 1998; Lee et al., 1998). DMSO inhibited metabolism of aniline, aminopyrine, 7-ethoxycoumarin and $p$-nitroanisole, and induced benzo(a)pyrene metabolism, in liver supernatant from rats pre- 
treated with Aroclor, but only inhibited metabolism of 7ethoxycoumarin, aminopyrine and benzo(a)pyrene in supernatant from rats treated with phenobarbital (Kawalec and Andrews, 1980). DMSO also inhibited the metabolism of benzo(a)pyrene in rabbit lung microsomes, although to a lesser extent than other solvents (Kontir et al., 1986). A dose of $1.5 \mathrm{ml} / \mathrm{kg}$ DMSO induced renal ethoxycoumarin-O-deethylase in rats, but the enzyme was inhibited when the solvent was added to renal microsomes in vitro (Zitting et al., 1983). However, it had no effect on hepatic CYP2A6, or renal NADPH-cytochrome c reductase (Zitting et al., 1983; Chauret et al., 1998). DMSO has been shown to inhibit bioactivation of sulindac in vitro and in vivo, in rats and humans, by competitive inhibition of sulfoxide reductase (Ratnayake et al., 1981; Swanson et al., 1981; Swanson et al., 1983a; Swanson et al., 1983b). The excipients sulfide metabolite also inhibited sulfide oxidases (Swanson et al., 1983b).

\subsubsection{Effects of DMSO on renal elimination}

DMSO also has the potential to affect renal elimination of drugs. The excipient has been reported to cause osmotic diuresis in humans (Muther and Bennett, 1980; Gunn and Acomb, 1986). However, DMSO did not affect plasma creatinine levels in rats, after dosing with $0.25-1 \mathrm{ml} / \mathrm{kg}$ for 10 days (Ali and Mousa, 2001), or in humans after dosing with $0.9 \mathrm{ml} / \mathrm{kg}$ for three days (Muther and 
Bennett, 1980). Pestel et al. (2006) reported that DMSO at a dose of $200 \mathrm{mg} / \mathrm{kg}$ i.v. did not affect urine volume in rats, but did cause protein to appear in the urine.

\subsubsection{Effects of DMSO on oral absorption}

The effects of oral doses of DMSO on oral absorption were assessed by Passananti et al. (1975), for salicylic acid, sulfanilamide and warfarin. No differences in plasma concentrations or $t_{1 / 2}$ were seen for salicylic acid or sulfanilamide (DMSO dose $200 \mathrm{mg} / \mathrm{kg}$ ). For the experiments with warfarin, the dose of DMSO was increased to $500 \mathrm{mg} / \mathrm{kg}$, and an additional $300 \mathrm{mg} / \mathrm{kg}$ dose of DMSO was given 10 minutes after drug administration. Changes were seen in the plasma concentration-time profile of warfarin; an initial lowering of plasma concentration was observed, followed by increased plasma concentrations. These changes were attributed to increased gastric $\mathrm{pH}$ and subsequently altered gastric emptying due to DMSO.

Warfarin $t_{1 / 2}$ was not significantly altered (Passananti et al., 1975). However, Pestel et al. (2006) reported that DMSO did not significantly affect gastric emptying at doses of up to $1000 \mathrm{mg} / \mathrm{kg}$ p.o., but did slow intestinal transit at this dose.

\subsubsection{Effects of DMSO on distribution}

Several studies suggest possible effects of DMSO on distribution. Gross et al. (1993) reported increased ${ }^{14} \mathrm{C}$ levels in the brain, heart 
and carcass of pregnant mice after administration of ${ }^{14} \mathrm{C}$-caffeine in 20\% DMSO, compared to distilled water. Kassell et al. (1983) reported that DMSO increased cerebral blood flow and reduced renal blood flow in dogs, at doses of $6 \mathrm{~g} / \mathrm{kg}$ and $2 \mathrm{~g} / \mathrm{kg}$, respectively. However, these effects were not statistically significant. Brink and Stein (1967) reported that DMSO increased brain and blood levels of ${ }^{14} \mathrm{C}$-pemoline in rats, compared to dosing in tragacanth suspension. A dose of $0.67 \mathrm{ml} / \mathrm{kg}$ DMSO increased blood levels of i.p.-administered ${ }^{14} \mathrm{C}$-pemoline by almost 2 -fold 30 minutes after administration, while brain concentrations increased by 2.4 -fold. Iwen and Miller (1986) reported that DMSO increased brain and serum levels of ketoconazole in mice. Mice were dosed orally with ketoconazole twice daily for three days, with some mice receiving $0.5 \mathrm{ml} 15 \%$ DMSO i.p. 30 minutes before ketoconazole, then $0.25 \mathrm{ml}$ i.v. after ketoconazole dosing. Brain and serum levels of ketoconazole were significantly increased 3 hours after dosing, by 9-fold and 3.5-fold respectively. This dose of DMSO was associated with a $9.8 \%$ mortality rate. However, Rubinstein and Lev-El (1980) reported that DMSO $(0.7 \mathrm{~g} / \mathrm{kg}$ for 10 days $)$ did not significantly affect the tissue distribution of gentamycin in rats, except for a 3.6fold increase in concentrations in the heart. DMSO $(0.9 \mathrm{ml} / \mathrm{kg})$ has also been reported to increase plasma levels of the acute-phase reactant $\alpha_{1}$-acid glycoprotein in rats (Ivanovic Matic et al., 2004), 
demonstrating that it has the potential to affect the plasma protein binding of drugs which bind to this protein.

\subsubsection{Ethanol}

\subsubsection{Effects of ethanol on metabolism}

Ethanol has been widely studied with respect to its effects on metabolism. In vitro, it has been shown to inhibit metabolism of aniline, aminopyrine, 7-ethoxycoumarin, p-nitroanisole, benzo(a)pyrene and morphine (Kawalec and Andrews, 1980; Kontir et al., 1986; Aasmundstad et al., 1996), and to alter the metabolite profiles of morphine and benzo(a)pyrene (Kontir et al., 1986; Aasmundstad et al., 1996). The solvent also induced metabolism of aniline in phenobarbital induced rat liver supernatant (Kawalec and Andrews, 1980). Ethanol is metabolised by CYP2E1, and can induce this enzyme (Rendic and Di Carlo, 1997).

Ethanol has also been reported to affect the metabolism and pharmacokinetics of drugs in vivo. Chronic ethanol feeding $(2 \mathrm{~g} / \mathrm{day}$ p.o. for 3 weeks) in rats has been shown to affect the metabolism of chloroform and carbon tetrachloride (Wang et al., 1997). Liver CYP450 content was increased by $36 \%$ after ethanol treatment, and in vitro metabolism of both compounds was increased in 
microsomes from ethanol-treated rats. In vivo, both compounds were administered by the p.o., i.p., and inhalation routes, at both high and low doses $(0.105$ and $1.675 \mathrm{mmol} / \mathrm{kg}$ p.o. and i.p., 50 and $500 \mathrm{ppm} / 6 \mathrm{~h}$ by inhalation). The AUC of carbon tetrachloride was significantly reduced after administration by all routes, but only at the lower doses tested. In contrast, chloroform AUCs were reduced for both doses tested by the p.o. route, but only at higher doses for the i.p. and inhalation routes. This was attributed to the metabolism of this compound being limited by hepatic blood flow at low doses. Peng et al. (1982) reported increased microsomal NDMA demethylase activity in rats, following chronic ethanol feeding. Feeding with 10 or $15 \%$ ethanol for 3 days increased NDMA demethylase activity by 4 -fold and 5.6-fold, respectively, with corresponding increases in CYP450 content of 38 and $59 \%$. In contrast, addition of ethanol to microsomes in vitro inhibited NDMA demethylase, thus highlighting the different results which can occur between in vitro and chronic in vivo study designs.

Ethanol $(1 \mathrm{~g} / \mathrm{kg})$ has also been reported to affect the pharmacokinetics of orally administered cocaine in dogs (Parker et al., 2002). Ethanol treatment caused a $75 \%$ reduction in $\mathrm{CL}, 4$-fold increases in bioavailability and AUC, and a 3 -fold increase in $\mathrm{C}_{\max }$. There was no effect on $t_{1 / 2}$. Different effects of ethanol on cocaine pharmacokinetics were seen in pigs receiving chronic ethanol 
treatment (1g/kg/day for 10 days) (Kambam et al., 1994). CL and $V$ of i.v. administered cocaine were increased by 3 -fold and $43 \%$, respectively, in the ethanol treated animals. AUC was reduced by $67 \%$, and $t_{1 / 2}$ by $47 \%$. These studies highlight that acute and chronic ethanol dosing can have different effects on pharmacokinetics.

\subsubsection{Effects of ethanol on hepatic blood flow}

A 6-hour infusion containing ethanol, started 3 hours before drug administration, increased the clearance of propoxyphene in rats (Oguma and Levy, 1981). As propoxyphene clearance is hepatic blood flow limited, this suggests that ethanol increased hepatic blood flow. When the drug was administered into the portal circulation, ethanol increased systemic availability of the drug, attributed to inhibited first-pass metabolism (Oguma and Levy, 1981). This inhibitory effect was only apparent after pre-systemic adminstration of propoxyphene, due to the blood flow-limited clearance of this drug. Other authors have also reported that ethanol increased propoxyphene blood concentrations, and reduced metabolite concentrations, after oral administration in rats and dogs (Olsen et al., 1983; Bodd et al., 1985); this would be consistent with the inhibited first-pass metabolism described by Oguma and Levy. 


\subsubsection{Effects of ethanol on renal elimination}

Ethanol can also affect the renal elimination of drugs. Grauer et al. (1987) demonstrated that ethanol (19.3mmol/kg i.v., 4 doses over $24 \mathrm{~h}$ ) reduced serum $t_{1 / 2}$ of ethylene glycol in dogs by $37 \%$, and increased the rate constant of urinary excretion (3-72 hours) by $70 \%$. Kortoupoulos et al. (1983) investigated the effects of ethanol on the pharmacokinetics of penicillin in rats. Dosing with $2 \mathrm{~g}$ ethanol i.p. significantly reduced serum penicillin concentrations at 15 and 30 minutes after drug administration. Brain levels were also significantly reduced at 15 minutes. Urine volume over $0-4$ hours was increased by $43 \%$ in the ethanol treated rats, accompanied by a $13 \%$ reduction (non-significant) in penicillin elimination into the urine in this period. Ethanol also has the potential to affect organic anion transport in the kidney. Kim et al. (1986) reported that ethanol inhibited $p$-aminohippurate uptake into rabbit kidney slices, but did not affect the uptake of the organic cation tetraethylammonium. 


\subsubsection{Propylene glycol}

\subsubsection{Effects of PG on metabolism}

Several workers have demonstrated

alteration of whole body pharmacokinetics

due to an inhibitory effect of propylene glycol (PG) on metabolism. Walters et al.

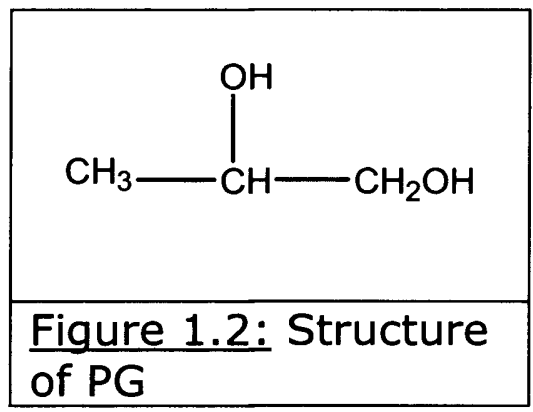

(1993) demonstrated increased blood levels of dramamine in animals previously exposed to PG, compared to naïve animals. This was attributed to inhibited hepatic metabolism, as the vehicle inhibited dramamine metabolism in microsomes (Walters et al., 1993). Dean and Stock (1974) investigated the effects of PG on metabolism in vitro and in vivo. In vitro, in liver microsomes from rats dosed with $4 \mathrm{ml} / \mathrm{kg}$ of the vehicle twice daily for 3 days, PG inhibited aminopyrine demethylation, increased aniline hydroxylation and $p$-nitroanisole metabolism, but had no significant effect on $p$-nitrobenzoic acid metabolism. CYP450 concentrations were not altered by this treatment. The effects could not be mimicked by adding the solvent to the incubation mixture in vitro. Metabolism of aniline and $p$-nitroanisole were also significantly increased after dosing with $1 \mathrm{ml} / \mathrm{kg}$ of the excipient twice dailly for three days. In vivo, PG increased hexobarbital sleeping times and zoxazolamine paralysis, suggesting inhibited metabolic clearance of these compounds. PG has also been shown to inhibit 
acetaminophen metabolism in vitro (Snawder et al., 1993). The excipient inhibited CYP2E1 but not CYP1A2. This was suggested as the mechanism by which PG protects from acetaminophen hepatotoxicity.

\subsubsection{Effects of $P G$ on distribution and renal excretion}

Oshiro et al. (1990) also demonstrated altered pharmacokinetics due to $P G$. The $t_{1 / 2}$ and $V$ of phenylbutazone were significantly increased (by $26 \%$ and $40 \%$ respectively) when the drug was administered in PG. There was no difference in CL or AUC between the formulations, suggesting that this may have been an effect on distribution rather than metabolism. PG has been reported to cause nephrotoxicity in patients receiving the excipient as an injection vehicle (Hayman et al., 2003; Yaucher et al., 2003), suggesting that the excipient could affect renal elimination of drugs.

\subsubsection{Effects of PG on oral absorption}

PG has been shown to affect brush border enzyme activities and nutrient uptake in the small intestine (Morshed et al., 1991). Uptake of calcium, D-glucose and certain amino acids were increased after chronic dosing for 20 or 30 days. In addition, activity of sucrase, lactase and $Y$-glutamyl transpeptidase were significantly increased, with various enzymes being affected after 10,20 or 30 days of dosing. No change in intestinal ultrastructure 
or effect on vesicular transport was observed. It is possible that intestinal absorption of drugs could thus be affected by the solvent. However, Cornaire et al. (2004) reported that PG (0.5\%) had no significant effects on transport of digoxin across an enverted rat gut sac in vitro. Also, Schulze et al. (2005) reported that a $2 \mathrm{~g}$ oral dose of the excipient did not significantly affect antipyrine or ampicillin bioavailability, or gastric emptying time or small intestinal transit time, in dogs.

\subsubsection{PEGs}

\subsubsection{Effects of PEGs on oral absorption}

PEG 400 has been shown to inhibit

intestinal P-Gp and CYP3A at

concentrations of $1 \%$ and above, in

the excised rat jejunum (Johnson et

al., 2002). However, these effects

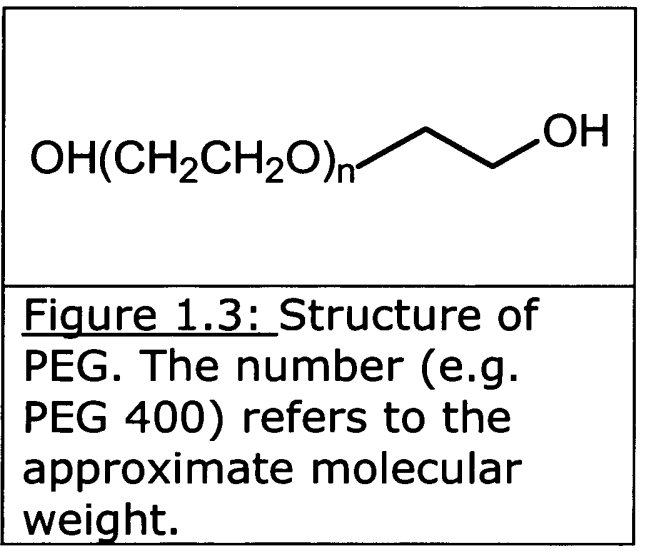

were not apparent at a concentration of $0.5 \%$, as PEG 400 did not significantly affect digoxin transport across everted rat gut sac in vitro at this concentration (Cornaire et al., 2004). Additionally, Hugger et al. (2002) demonstrated that PEG-300 inhibited P-Gp in both Caco-2 monolayers and MDR1-MDCK cells, at a concentration of $20 \%$. An increase in the oral absorption of P-Gp substrates 
administered in PEG therefore appears possible, due to reduced drug efflux into the intestinal lumen, and also of CYP3A substrates, due to reduced intestinal metabolism. Absorption of centbutindole through the gastric mucosa, despite predictions to the contrary based on $\mathrm{pH}$ and ionisation state, was attributed to the presence of 20\% PEG 600 in the formulation (Issar et al., 2003). However, no comparative work was done with other formulations so this assumption must be treated with caution.

PEG can also reduce oral absorption of drugs. PEG 400 (10g) reduced oral bioavailability $(F)$, MAT and $C_{\max }$ of ranitidine in humans, due to reduction in small intestinal transit time, and possibly influx of fluid into the gut lumen due to osmotic effects of PEG (Basit et al., 2002). Lower doses of $2.5 \mathrm{~g}$ and $5 \mathrm{~g}$ significantly reduced small intestinal transit time, and reduced urinary excretion of ranitidine, used as a measure of drug absorption after oral dosing, by $38 \%$ (Schulze et al., 2003). A $1 \mathrm{~g}$ dose increased the amount of ranitidine excreted in urine after oral dosing by $41 \%$. The excipient did not affect gastric emptying at $1 \mathrm{~g}, 2.4 \mathrm{~g}$ or $5 \mathrm{~g}$ doses (Schulze et al., 2003). However, a 1g dose of PEG 400 did not significantly affect gastric emptying time, small intestinal transit time, or bioavailability of antipyrine or ampicillin in dogs (Schulze et al., 2005). Pestel et al. (2006) reported that PEG 400 significantly reduced gastric emptying but did not affect intestinal transit in rats 
at a dose of $\quad 2 \mathrm{~g} / \mathrm{kg} \mathrm{p.o.,} \mathrm{and} \mathrm{caused} \mathrm{liquid} \mathrm{accumulation} \mathrm{in} \mathrm{the}$ intestinal contents.

\subsubsection{Effects of PEGs on renal elimination}

Pestel et al. (2006) reported that dosing with $1000 \mathrm{mg} / \mathrm{kg}$ PEG 400 i.p. reduced urine volume over the period $0-4$ hours, and there was an increase in urinary creatinine excretion at a dose of $2000 \mathrm{mg} / \mathrm{kg}$ by the i.p. but not i.v. route. Protein appeared in urine after dosing with $2000 \mathrm{mg} / \mathrm{kg}$ i.v. This suggests that PEG 400 has the potential to affect the renal elimination of drugs at these doses. Laine et al. (1995) attributed acute tubular necrosis seen in an intensive care patient to PEG 400 . The patient received a cumulative i.v. dose of $220 \mathrm{ml}$ of the excipient over 43 days, as a vehicle for lorazepam. Administration of vancomycin was also identified as a possible contributing factor. This is further evidence of the potential of PEG 400 to affect renal function.

PEG as a cosolvent therefore has the potential to affect the absorption and distribution of drugs, but little work has been done to investigate this; the focus has been on drug delivery systems using PEG conjugates rather than PEG alone. 


\subsubsection{Other cosolvents}

\subsubsection{Effects of other cosolvents on metabolism}

Effects of dimethylacetamide (DMA) on metabolism have been reported.

Menicagli et al. (1994) reported that

treatment with $300 \mathrm{mg} / \mathrm{kg}$ DMA for

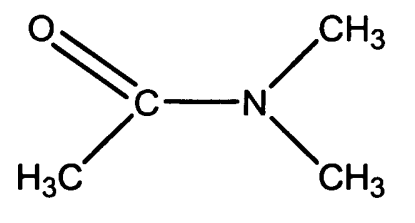

Figure 1.4: Structure of $\mathrm{N}, \mathrm{N}$-dimethylacetamide.

three days did not significantly affect the activity of CYP450 or phase 2 enzymes in rat liver microsomes. Tolando et al. (2001) reported that DMA caused dose-dependant loss of CYP450 in liver microsomes from pyridine-treated rats. This was attributed to inactivation of haem by a metabolite of the solvent.

Glycofurol has been shown to alter drug pharmacokinetics by inhibiting hepatic metabolism. Loscher et al. (1995) compared the intravenous

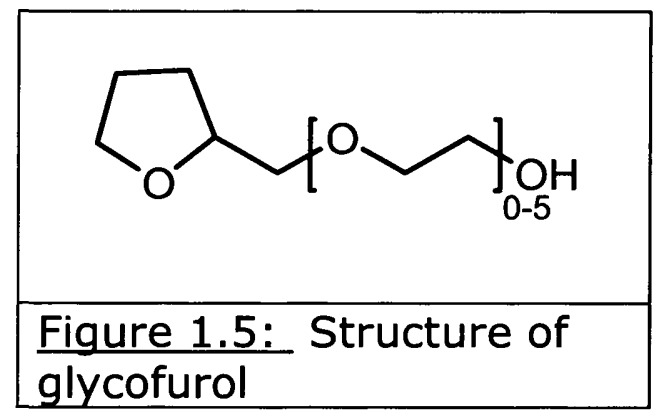
pharmacokinetics of carbamazepine in glycofurol $(325 \mathrm{mg} / \mathrm{kg}$ ) and a hydroxypropyl- $\beta$-cyclodextrin solution $(100 \mathrm{mg} / \mathrm{kg})$ in dogs. The glycofurol vehicle gave significant increases in $t_{1 / 2}(1.7$-fold increase), $C_{\max }$, and $A \cup C$, compared to the cyclodextrin solution, and reduced formation of the epoxide metabolite. Similarly, glycofurol was shown to decrease elimination of zoxazolamine and hexobarbital in rats (Yasaka et al., 1978). A single i.p. dose of 
$1.25 \mathrm{ml} / \mathrm{kg}$ reduced the clearance and increased plasma and brain levels of both compounds, and increased hexobarbital sleeping times and zoxazolamine paralysis. In vitro metabolism of both compounds was inhibited when glycofurol was added to microsomal preparations. Repeated dosing ( $1 \mathrm{ml} / \mathrm{kg} / \mathrm{bd}$ for four days) appeared to have an enzyme-inducing effect, reducing zoxazolamine paralysis and hexobarbital sleeping times and increasing their metabolism in microsomes from the dosed rats, as well as CYP450 levels (Yasaka et al., 1978).

\subsection{Surfactants and emulsions}

\subsubsection{Cremophor}

$$
\begin{aligned}
& \mathrm{CH}_{2}-\mathrm{O}-\left(\mathrm{CH}_{2} \mathrm{CH}_{2} \mathrm{O}\right)_{x}-\mathrm{CH}_{2} \mathrm{OO}\left(\mathrm{CH}_{2}\right)_{7}-\mathrm{CH}=\mathrm{CH}-\mathrm{CH}_{2}-\mathrm{CHOH}-\left(\mathrm{CH}_{2}\right)_{5}-\mathrm{CH}_{3} \\
& \text { I } \mathrm{H}-\mathrm{O}-\left(\mathrm{CH}_{2} \mathrm{CH}_{2} \mathrm{O}\right)_{y}-\mathrm{CH}_{2} \mathrm{OO}\left(\mathrm{CH}_{2}\right)_{7}-\mathrm{CH}=\mathrm{CH}-\mathrm{CH}_{2}-\mathrm{CHOH}-\left(\mathrm{CH}_{2}\right)_{5}-\mathrm{CH}_{3} \\
& \text { I } \mathrm{CH}_{2}-\mathrm{O}-\left(\mathrm{CH}_{2} \mathrm{CH}_{2} \mathrm{O}\right)_{2}-\mathrm{CH}_{2} \mathrm{OO}\left(\mathrm{CH}_{2}\right)_{7}-\mathrm{CH}=\mathrm{CH}-\mathrm{CH}_{2}-\mathrm{CHOH}-\left(\mathrm{CH}_{2}\right)_{5}-\mathrm{CH}_{3}
\end{aligned}
$$

Figure 1.6: structure of polyoxyethyleneglycerol triricinoleate, the principal component of Cremophor EL (Gelderblom et al., 2001).

Cremophor is produced by reacting castor oil with ethylene oxide (BASF, 2004). The structure of the principal component of Cremophor EL is shown in Figure 1.6. The effects of this excipient 
on pharmacokinetics have previously been reviewed by ten Tije et al. (2003), Bittner and Mountfield (2002), and Gelderblom et al. (2001).

\subsubsection{Effects of Cremophor on pharmacokinetics}

Cremophor EL is used in the formulation of the anticancer drug paclitaxel, and has been shown to have profound effects on its pharmacokinetics. Sparreboom et al. (1996) demonstrated that the non-linear pharmacokinetics of paclitaxel were in fact due to Cremophor in the commercially available formulation, Taxol. Administration of increasing doses of paclitaxel to mice as Taxol gave disproportionate increases in $\mathrm{C}_{\max }$ and $\mathrm{AUC}$, and reductions in CL. However, when the drug was administered in DMA, CL did not alter with increasing dose, and $\mathrm{C}_{\max }$ increased proportionally. $\mathrm{C}_{\max }$ and CL obtained after administration in Tween corresponded to those obtained for the same paclitaxel dose in DMA. Supplementing Taxol with extra Cremophor also increased $\mathrm{C}_{\max }$ and reduced $\mathrm{CL}$. However, tissue distribution did not appear to be affected by formulation. The $\mathrm{CL}$ of Cremophor itself decreased with increasing doses. The authors concluded that Cremophor was responsible for the apparent non-linear pharmacokinetics of paclitaxel.

Administration of paclitaxel in a liposomal formulation resulted in significant alterations in the tissue distribution of the drug, 
compared to administration a Cremophor-EL based formulation (Fetterly and Straubinger, 2003). Paclitaxel AUCs in kidney, muscle, brain, bone marrow, skin, and adipose tissue were significantly increased by dosing in Cremophor, while spleen AUC was reduced. AUCs in blood, liver and lung were not significantly affected. These changes in tissue distribution between the formulations were reflected in increases in $\mathrm{V}_{\mathrm{t}}(2$-fold $)$ and $\mathrm{CL}_{d}(5.8$-fold $)$, and a 10.6fold reduction in $t_{1 / 2 \alpha}$, while total $C L$ and $t_{1 / 2 \beta}$ were not affected. When paclitaxel was administered in polymeric micelles, plasma levels and AUC were reduced compared to dosing in Cremophor, but levels in the liver, kidney, spleen, lungs and heart were not affected (Kim et al., 2001).

Webster et al. (1996) reported that Cremophor significantly affected the pharmacokinetics of doxorubicin and its metabolite doxorubicinol in mice. The AUCs of doxorubicin and doxorubicinol were increased by 2 -fold and 2.5-fold, respectively. Doxorubicin $V$ was significantly reduced by $63 \%$. Terminal $t_{1 / 2}$ of the drug and metabolite, and the ratio of doxorubicinol AUC to doxorubicin AUC, were not affected. The authors suggested altered distribution to the liver, or inhibition of biliary elimination, as possible mechanisms for the changes seen. Administration of the photosensitizer SiNC 8 in Cremophor EL resulted in higher plasma levels and increased $t_{1 / 2}$ ( $7.6 \mathrm{~h}$ vs $<1 \mathrm{~h}$ ) compared to administration in Solutol HS15 
(Brasseur et al., 1995). Cremophor also affected the pharmacokinetics of i.p. administered docetaxel in rats; $k_{a}$ was reduced by $58 \%$, and $k_{e}$ by $66 \%$, after dosing in $4.2 \%$ of the excipient (Yokogawa et al., 2004). Jin et al. (2005) investigated the effects of Cremophor EL on the pharmacokinetics of cyclosporin in rats. Blood and plasma AUCs were significantly increased with increasing doses of the excipient; 1.8 and 4.7-fold increases in plasma AUC were seen after dosing in 16 or $30 \%$ Cremophor, compared to $4.3 \%$. CL and $V_{s s}$ decreased with increasing Cremophor dose, although the reduction seen in plasma CL was not significant with $16 \%$ Cremophor. Interestingly, when $30 \%$ Cremophor was administered 30 minutes after the drug, cyclosporin blood and plasma levels subsequently increased.

\subsubsection{Effects of Cremophor on distribution in blood}

There has been much interest in the effects of Cremophor on the blood distribution of drugs. Sparreboom et al. (1999) reported that Cremophor EL reduced paclitaxel accumulation in erythrocytes, by entrapment in Cremophor micelles, thus reducing the free fraction of paclitaxel. Jin et al. (2005) reported that the blood to plasma concentration ratio of cyclosporin was reduced when dosed in $16 \%$ and $30 \%$ Cremophor, compared to $4.3 \%$ Cremophor. Cremophor has also been shown to alter the electrophoretic mobility of serum lipoproteins in mice and humans (Sykes et al., 1994; Woodburn et 
al., 1994; Woodburn et al., 1995). The excipient also caused the appearance of lipoprotein degradation products, which bound paclitaxel and C8KC (Sykes et al., 1994; Woodburn et al., 1994), and altered the protein binding pattern of these drugs. However, the excipient did not alter the protein binding of mesoporphyrin (Woodburn et al., 1995).

\subsubsection{Effects of Cremophor on hepatic elimination}

Effects of Cremophor on in vitro metabolism have also been reported. Cremophor EL reduced the clearance of the CYP3A substrate midazolam in rat hepatocytes and rat liver microsomes, at concentrations of $0.03 \%$ and $0.3 \%$ (Bravo Gonzalez et al., 2004b). Cremophor RH40 inhibited CYP3A in hepatic microsomes (Wandel et al., 2003).

Cremophor has been shown to inhibit elimination of etoposide and paclitaxel in the isolated perfused rat liver (Ellis et al., 1996; Ellis and Webster, 1999). In the etoposide study, Cremophor increased etoposide perfusate concentration, increased AUC by 4.6 -fold, and reduced $C L$ by $77 \%, V$ by $85 \%$, and $t_{1 / 2}$ by $35 \%$. Biliary $C L$ and the biliary excretion rate constant were reduced by $81 \%$ and $39 \%$, respectively. Similarly, for paclitaxel, Cremophor increased perfusate concentrations, increased $A \cup C$ by 9 -fold, and reduced $\mathrm{CL}$ and biliary CL by $89 \%$. $V$ was also reduced by $42 \%$, but this was 
not significant. In contrast to etoposide, $t_{1 / 2}$ was significantly increased by 5-fold. A ten-fold reduction in the Cremophor dose gave a significant increase in paclitaxel AUC (2-fold) and reduction in $\mathrm{CL}(47 \%)$ only (Ellis and Webster, 1999).

\subsubsection{Effects of Cremophor on renal elimination}

Cremophor has also been shown to affect renal elimination of drugs. Speeg et al. (1992) reported that Cremophor EL increased the $C L_{R}$ and secretory ratio of colchicine in rats by $14 \%$. Urinary flow was transiently increased, but the excipient did not affect GFR. In contrast, Tibell et al. (1993) reported that Cremophor EL caused a significant $26 \%$ reduction in GFR in rats. The lack of effect on GFR reported by Speeg et al. may be due to the lower dose of excipient used in their study; Tibell et al. (1993) dosed with $0.26 \mathrm{~g} / \mathrm{kg}$ bolus then $0.26 \mathrm{~g} / \mathrm{kg} / \mathrm{hr}$ for $2 \mathrm{hrs}$, compared to $130 \mathrm{mg} / \mathrm{kg}$ in Speeg et al.'s study. Besarab et al. (1987) reported that Cremophor reduced GFR and renal blood flow (due to vasoconstriction) in the isolated perfused rat kidney. The authors of the latter two studies suggested that Cremophor may contribute to the nephrotoxic side-effects of cyclosporin in the Cremophor-containing formulation Sandimmun ${ }^{\circledR}$.

\subsubsection{Effects of Cremophor on $P-G p$}

In vitro, Cremophor EL has been shown to inhibit $\mathrm{P}-\mathrm{Gp}$ in Caco-2 and P338 cells, and Cremophor RH40 in Caco-2 cells (Hugger et al., 
2002; Bogman et al., 2003; Wandel et al., 2003). However, the excipient has a very low volume of distribution, appearing to be limited to the central compartment - this is likely to limit its ability to inhibit P-Gp in tissues in vivo (Sparreboom et al., 1998).

\subsubsection{Effects of Cremophor on oral absorption}

Inhibition of P-Gp and CYP3A would lead us to expect that Cremophor could increase the oral absorption of drugs which were substrates for these in the small intestine. In line with this, Cremophor EL was shown to increase absorption of the P-Gp substrate digoxin across an everted rat gut sac in vitro, at concentrations of $0.05 \%$ and above (Cornaire et al., 2000; Cornaire et al., 2004). In vivo, Cremophor appeared to reduce digoxin absorption in rats, reducing digoxin $\mathrm{AUC}_{0-40}$ and $A \cup \mathrm{C}_{0-480}$ by 42 and $49 \%$, respectively (Cornaire et al., 2004); however, no statistical analysis of this is reported. Cremophor RH40 increased the oral absorption of digoxin in humans. (Tayrouz et al., 2003). Subjects were dosed with $600 \mathrm{mg}$ of the excipient three times a day, for 16 hours before and 9 days after drug administration, including one dose administered with the drug. Digoxin $\mathrm{C}_{\max }$ and $\mathrm{AUC}_{0-5 \mathrm{~h}}$ were increased by $22 \%$, attributed to inhibition of intestinal P-Gp by Cremophor RH40. Martin-Facklam et al. (2002) reported that Cremophor EL increased the oral absorption of the P-Gp and CYP3A substrate saquinavir in humans. $\mathrm{C}_{\max }$ and $A \cup \mathrm{C}_{0-4 \mathrm{~h}}$ were increased by 
13-fold, and $A \cup C_{0-\infty}$ by 5 -fold, after dosing with $5000 \mathrm{mg}$ of the excipient.

Cremophor has also been reported to impair the oral absorption of paclitaxel in mice and humans. In P-Gp knockout mice, a 7-fold increase in Cremophor EL dose reduced paclitaxel AUC by $36 \%$ and $\mathrm{C}_{\max }$ by $75 \%$, and increased excretion of the drug in faeces (Bardelmeijer et al., 2002). Similarly, in humans pre-treated with cyclosporin A, administration of the drug in Cremophor gave significantly lower $\mathrm{C}_{\max }$ and $\mathrm{AUC}$, compared to administration in Tween (Malingre et al., 2001). Faecal excretion of paclitaxel was also increased, although not significantly, and the amount of the drug excreted by this route significantly correlated with the amount of Cremophor excreted here. The AUC and $\mathrm{C}_{\max }$ of the cyclosporin pre-treatment were also reduced by Cremophor. These effects were attributed to entrapment of drug in Cremophor EL micelles in the intestine. It must be bourne in mind that these effects were seen in subjects where P-Gp was absent or inhibited. It would be of interest to see the effects of Cremophor on paclitaxel absorption when P-Gp was active. Cremophor therefore appears to have the potential to increase oral absorption by inhibition of intestinal P-Gp and CYP3A, and also to reduce it by micellar entrapment. 


\subsubsection{Tween}

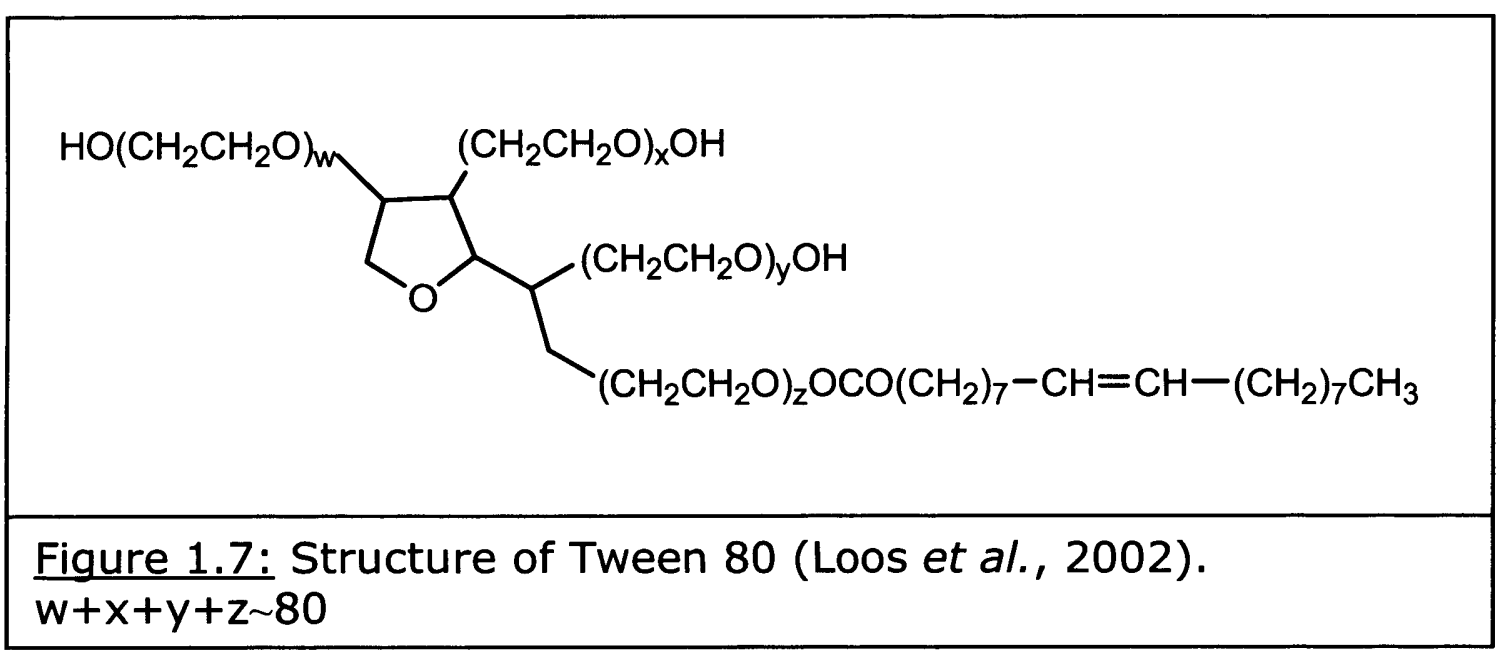

The structure of Tween (polysorbate) 80 is shown in Figure 1.7. Its effects on drug disposition have previously been reviewed by ten Tije et al. (2003).

\subsubsection{Effects of Tween on P-Gp}

Like Cremophor, Tween 80 can inhibit P-Gp in vitro (Hugger et al., 2002; Bogman et al., 2003). In agreement with this property, both Tween 20 and Tween 80 increased absorption of the P-Gp substrate digoxin across an everted rat gut sac in vitro, at a concentration of 0.5\% (Cornaire et al., 2000; Cornaire et al., 2004). Tween 80 also reduced the ratio of serosal-mucosal transport to mucosal-serosal transport of rhodamine-123 across rat jejunal membrane in vitro, again suggesting inhibition of P-Gp (Shono et al., 2004). 
These findings were confirmed in vivo by Zhang et al. (2003), who demonstrated increased $A \cup C$ and $C_{\max }$ for orally administered digoxin in rats when co-administered with Tween. Increasing the dose of Tween increased the extent of absorption; $A U C$ and $C_{\max }$ were increased by $30 \%$ and $163 \%$ respectively after dosing in $1 \%$ Tween, and by $61 \%$ and $161 \%$ after dosing in a $10 \%$ solution. This effect was attributed to intestinal P-Gp inhibition, as when Tween was administered orally and digoxin administered i.v., no significant change in $\mathrm{CL}$ or AUC occurred. The same authors also demonstrated a $36 \%$ increase in digoxin AUC and a $24 \%$ reduction in $\mathrm{CL}$ when both digoxin and Tween were administered i.v. This was thought to be due to inhibition of P-Gp-mediated billiary and renal efflux of the drug (Zhang et al., 2003). Pestel et al. (2006) reported that oral dosing with $50 \mathrm{mg} / \mathrm{kg}$ Tween 80 did not significantly affect gastric emptying or intestinal transit in rats, suggesting that the excipient would not affect the oral absorption of drugs in this way.

\subsubsection{Effects of Tween on elimination}

Azmin et al. (1985) reported that Tween altered methotrexate elimination in the mouse after i.v. administration. The amount of methotrexate eliminated in the urine was initially reduced by around $70 \%$ over the $0-4$ hour period, then increased by around 5fold over the 4-8 hour period. Elimination into faeces was increased by 1.7-fold. The authors suggested that Tween increased the 
(overall) renal and biliary excretion of methotrexate; however, there was only a very slight and non-significant reduction in drug plasma levels. No effects on elimination were apparent after oral dosing; there was no discernable change in urinary excretion, and only a $14 \%$ increase in the amount of drug in faeces.

Enzyme inhibition has also been demonstrated for Tween. Both Tween 20 and Tween 80 inhibited human CDNA expressed CYP3A4 at concentrations of $0.005 \%$ and above (Mountfield et al., 2000). Tween 80 also reduced the clearance of the CYP3A substrate midazolam in rat liver microsomes at concentrations of $0.003 \%$ and above, and in hepatocytes at $0.03 \%$ and above (Bravo Gonzalez et al., 2004b). Ellis et al. (1996) reported that Tween 80 inhibited etoposide elimination in the isolated perfused rat liver. Tween gave higher perfusate concentrations of the drug, and caused statistically significant reductions in $C L(38 \%), V(66 \%)$, and $t_{1 / 2}(44 \%)$. The elimination profile from perfusate appeared to change from biphasic to monophasic. The rate constant for biliary excretion was also reduced by $29 \%$, although the total amount eliminated in bile was not affected, as the preparation appeared to recover from this effect over time.

The ability of Tween to affect pharmacokinetics was called into question by Van Tellingen et al. (1999), due to the pharmacokinetic 
properties of Tween. They demonstrated that plasma levels of the excipient declined rapidly in vivo; in mice, the plasma concentration fell from above $0.7 \%$ after dosing to less than $0.05 \% \mathrm{v} / \mathrm{v}$ in 15 minutes, and then declined more slowly. Unfortunately no half-lives or rate constants to describe this are reported. In humans, plasma levels of Tween were undetectable shortly after receiving an i.v. infusion of docetaxel containing the excipient. Tween was broken down by esterases in vitro, and this was suggested as the mechanism for the decline in plasma levels seen in vivo. The authors concluded that the rapid fall in Tween plasma levels made it unlikely that the vehicle had affected the pharmacokinetics of coadministered docetaxel, but no comparator vehicle was used to test this hypothesis. Indeed, the work described above demonstrates that Tween can affect i.v. pharmacokinetics.

\subsubsection{Effects of Tween on distribution}

Effects of Tween on distribution have also been reported. Like Cremophor, Tween can affect plasma protein binding, as demonstrated by Loos et al. (2003) for docetaxel. Tween 80 increased $\mathrm{f}_{\mathrm{u}}$ both in vitro and in vivo in human plasma. In vitro at low Tween concentrations a slight decrease in $f_{u}$ was observed, whereas at higher concentrations $f_{u}$ was increased. This biphasic in vitro relationship was attributed to two mechanisms: at low concentrations of Tween, micellar entrapment predominates which 
decreases $f_{u} ;$ at higher concentrations, displacement from binding sites predominates which increases $f_{u}$. Plasma $\alpha_{1}$-AGP levels were significantly related to $f_{u}$, suggesting that this is the protein involved in the binding effects of Tween (Loos et al., 2003). Tween has also been shown to reduce the blood to plasma concentration ratio of docetaxel in rat blood in vitro, at concentrations of $1 \%$ and above (Yokogawa et al., 2004).

Colombo et al. (1999) investigated the effects of Tween on doxorubicin distribution in mice. Doxorubicin levels in the spleen were increased by about $60 \%$. Smaller but significant increases were also seen in the heart and lungs at 8 hours post-dose, and in the liver and serum at $\mathbf{2 4}$ hours. Tween also significantly reduced levels of the aglycone metabolite in the kidney.

Tween has also been reported to affect the uptake of drugs into the brain. The study by Azmin et al. (1985) described above also reported significantly increased brain uptake of methotrexate in the presence of Tween after both oral and i.v. administration. The fact that this effect was also evident after oral administration of Tween is of interest, as this suggests that the surfactant, or a component of it, was orally absorbed in order to affect the brain uptake of the drug. The authors point out that the increase in brain levels seen after i.v. administration, where there was no increase in drug 
plasma levels, suggests that the effects seen after oral dosing are not just due to increased plasma levels of the drug. Dimitrijevic et al. (2001) reported increased brain entry of vigabatrin due to Tween. Although no vigabatrin was detected in the brain, parameters indicative of its presence there, i.e. brain GABA levels and rectal temperature lowering, were significantly increased when the drug was administered with Tween, compared to administration in saline. This led the authors to conclude that brain uptake of vigabatrin had been enhanced by the surfactant.

Gulyaev et al. (1999) reported that brain levels of doxorubicin were undetectable after dosing in saline or $1 \%$ Tween 80 , suggesting that the excipient did not increase brain uptake. However, there were some significant differences in liver, spleen, lung and kidney levels of doxorubicin between the saline and Tween solutions. Ten minutes after dosing, doxorubicin levels in the liver, kidneys and spleen were significantly increased by $1.5-2.4$ fold, and lung levels were significantly reduced by $36 \%$. However, these differences were only apparrent at 1 hour for the liver and spleen. Overall, levels of doxorubicin in a particular tissue were not consistently enhanced or reduced by Tween over the whole eight hour sampling period, although this may not be expected due to the rapid decline in Tween plasma levels. 
A substantial body of work exists describing the ability of nanoparticles coated with Tween 80 to increase the brain uptake of a variety of compounds, reviewed by Kreuter (2004). This material will not be covered here, as it represents a drug delivery system specifically designed to alter drug distribution, rather than the effects that might be seen after dosing of a compound in a Tween solution in an early pharmacokinetic study.

\subsubsection{Effects of Tween on pharmacokinetics after i.p.}

\section{administration}

Tween has also been reported to affect drug pharmacokinetics after i.p. administration. Harrison et al. (1981) reported that Tween increased plasma concentrations of adriamycin when administered intraperitoneally to mice, giving significantly higher concentrations at 1 and 2 hours post treatment. This was found to correspond to an increase in packed cell volume, signifying a reduction in plasma volume, which was thought to be due to the osmotic effects of Tween in the peritoneal cavity. Tween has also been shown to affect the pharmacokinetics of docetaxel after i.p. administration in rats. Dosing in $7.5 \%$ Tween reduced $k_{a}$ from the peritoneal cavity by $67 \%$, and reduced $k_{e}$ from plasma by $68 \%$ (Yokogawa et al., 2004). Pestel et al. (2006) reported renal effects of Tween after i.p. dosing. The excipient significantly reduced urine volume between 0 and 4 
hours after dosing in rats, and increased urine volume between 4 and 8 hours.

\section{3 .3 Solutol}

Solutol HS15 consists of 12-hydroxystearic acid mono-esters and di-esters, and a proportion of free PEG ( $30 \%)$ (BASF, 2004). The structures of the principal components of Solutol HS15 are shown in Figure 1.8 .

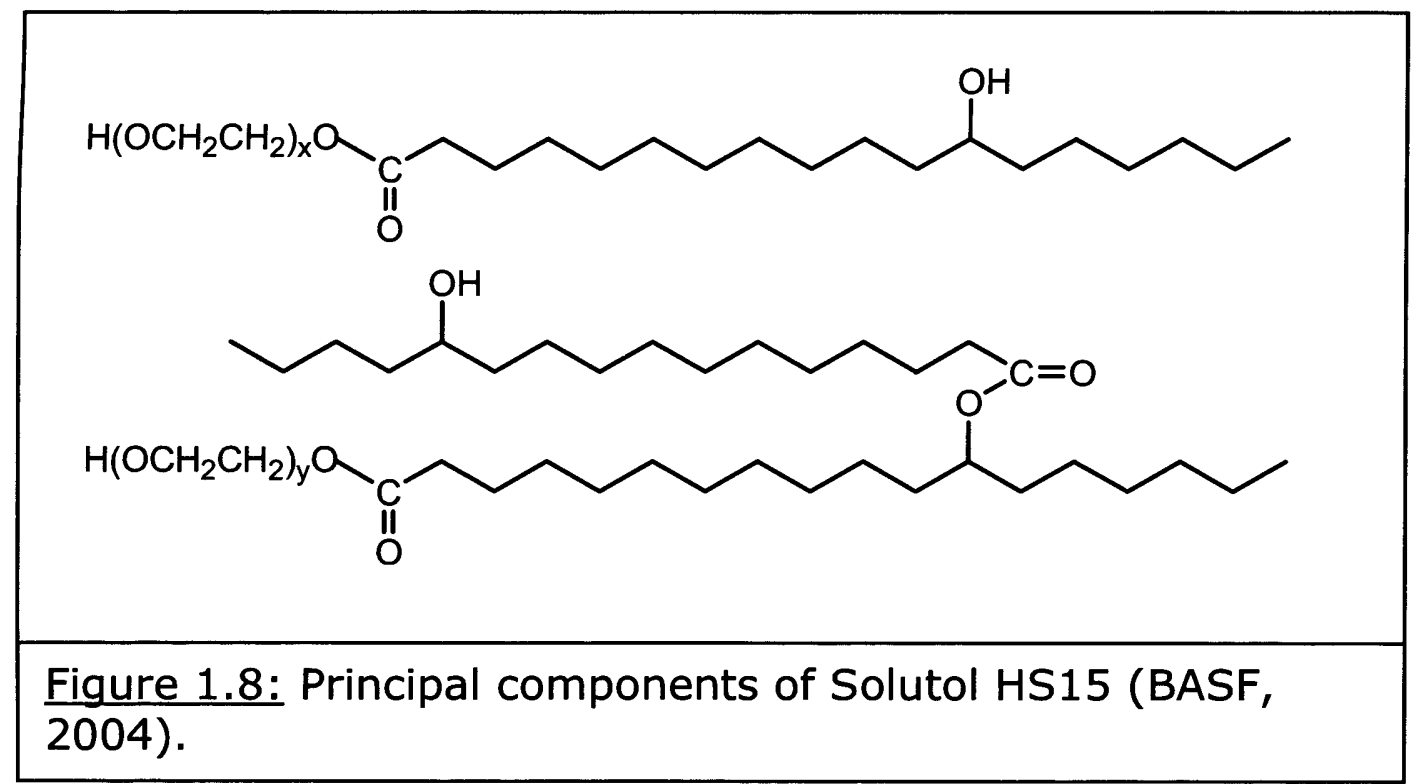

\subsubsection{Effects of Solutol on metabolism}

Effects of Solutol on metabolism have been demonstrated in vitro.

Solutol HS15 inhibited midazolam clearance in rat hepatocytes at concentrations of $0.003 \%$ and above, and in rat liver microsomes at 
concentrations of $0.03 \%$ and above (Bravo Gonzalez et al., 2001; Bravo Gonzalez et al., 2004b). Inhibition of CYP3A or binding of the drug to the excipient were suggested as possible mechanisms for this effect. However, the excipient did not affect midazolam pharmacokinetics in the rat (Bittner et al., 2003a). Dosing in 5\% Solutol did not affect midazolam $\mathrm{C}_{\max }, \mathrm{AUC}_{0-\infty}, \mathrm{A}_{\mathrm{e}}$, or $\mathrm{CL}$. Nor were the $\mathrm{C}_{\max }$ or $\mathrm{AUC}_{0-\infty}$ of its major metabolites affected. The authors ascribed this lack of effect to the high hepatic extraction ratio of midazolam, so that the weak enzyme inhibitor Solutol had no effect on in vivo clearance.

\subsubsection{Effects of Solutol on in vivo pharmacokinetics}

Solutol did have a profound effect on the pharmacokinetics of the water soluble drug colchicine in rats (Bittner et al., 2003b). A 5\% solution of Solutol HS15 increased $\mathrm{C}_{\max } 2.4$-fold and reduced CL by $55 \%$, compared to administration in saline. $V$ was reduced by $58 \%$ in the presence of Solutol; however, this was not significant. The excipient also substantially increased the amount of colchicine excreted into urine, by 35 -fold. Solutol also reduced in vitro $C L$ of colchicine in rat hepatocytes, at concentrations of $0.003 \%$ and above. Inhibited metabolism was suggested as a likely cause of the excipients effects on colchicine pharmacokinetics. Desrayaud et al. (1997) investigated the effects of the P-Gp inhibitor SDZ PSC 833 on the pharmacokinetics of colchicine in rats. They reported a 2.2- 
fold increase in $C_{\max }, 2.5$-fold increase in $\mathrm{AUC}_{0-6 \mathrm{~h}}$, and a $47 \%$ reduction in $C L . V_{\beta}$ was reduced by $18 \%$, which was not significant. These effects parallel those seen in Bittner et al's study, demonstrating that P-Gp inhibition by Solutol could be responsible, at least in part, for the effects seen. P-Gp inhibition by the excipient has been demonstrated in vitro; Solutol has been reported to reduce the IC50 of colchicine, vinblastine and doxorubicin, and increase the accumulation of rhodamine 123 and daunorubicin (Coon et al., 1991; Woodcock et al., 1992).

Solutol also significantly affected the pharmacokinetics of intravenously administered colchicine when the excipient was administered orally (Bittner et al., 2005). Oral dosing with Solutol 20 minutes before drug administration gave 2 -fold increases in $\mathrm{AUC}_{0-\infty}$ and $\mathrm{C}_{\max }$, and a $48 \%$ reduction in $\mathrm{CL}$. Urinary elimination of the drug was also affected; there was a 21 -fold increase in the elimination of unchanged colchicine by this route, and a 2.3 fold increase in urinary eliminiation of its demethyl metabolite (not significant). The changes seen here parallel those seen after i.v. dosing of Solutol described above, but are smaller in magnitude. The authors attribute the changes seen to oral absorption of Solutol or its metabolites, and suggest that the increased urinary elimination could be due to formation of a complex between the drug and excipient (or one of its metabolites) (Bittner et al., 2005). 


\subsubsection{Effects of Solutol on plasma protein binding}

Alteration of plasma protein binding by Solutol has been

demonstrated (Woodburn et al., 1995). Solutol altered the in vitro plasma protein binding of a photosensitizing agent (C8KC) compared to Cremophor, and reduced electrophoretic mobility of HDL and LDL. After dosing to mice, new lipoprotein species were observed. Solutol and the photosensitizer had similar plasma halflives in mice, leading the authors to suggest that the fate of the excipient was a determinant of the half-life of the drug. However, Solutol did not affect the in vitro protein binding of mesoporphyrin (Woodburn et al., 1995) or colchicine (Bittner et al., 2003b).

\subsubsection{Effects of Solutol on oral absorption}

Solutol has been shown to increase oral absorption of poorly soluble drugs. A formulation of cyclosporin A containing Solutol HS15, Labrafil M2125CS and oleic acid gave two-fold higher oral bioavailability compared to an aqueous microsuspension. $\mathrm{C}_{\max }$ and AUC were increased by 1.6 - and 1.7-fold, respectively, while $t_{1 / 2}$ and $t_{\max }$ were unchanged. This effect was thought to be predominantly due to increased solubility of the cyclosporin in the intestinal fluid, although inhibition of CYP3A and P-Gp may have played a part (Bravo Gonzalez et al., 2002). Effects of Solutol on the oral absorption of water soluble drugs have also been 
demonstrated. Bittner et al. (2002) investigated the effects of Solutol on the oral absorption of colchicine. A 4-fold increase in AUC was observed when the drug was administered in a $10 \%$ solution of Solutol, compared to saline. $\mathrm{C}_{\max }$ was increased and $\mathrm{CL} / \mathrm{F}$ decreased by 3.8- and 3.6-fold, respectively. The high water solubility of colchicine means that these effects are unlikely to be due to increased drug solubility in the intestinal fluids due to Solutol. Inhibition of P-Gp and/or CYP450 are suggested as possible mechanisms; the authors concluded that CYP450 inhibition is likely to be the major mechanism of enhanced absorption in this case, as colchicine was mostly absorbed in the upper GI tract, where CYP450 concentrations are high and P-Gp concentrations are low (Bittner et al., 2002). Cornaire et al. (2004) reported that Solutol HS15 significantly increased digoxin transport across an everted rat gut sac in vitro. Transport was increased by $2.2,1.8$ and 2.4 -fold, at concentrations of $0.05 \% 0.1$, and $0.5 \%$, respectively. This further demonstrates the ability of this excipient to affect drug absorption.

\subsubsection{Other emulsion systems}

\subsubsection{Effects of other emulsion systems on pharmacokinetics}

Propofol is usually administered in a lipid emulsion composed of soybean oil and lecithin, similar to Intralipid. Dutta and Ebling 
(1998a) reported significantly higher distribution volumes (3-fold increase in $V_{s s}, 12$-fold increase in $V_{c}$ ) and lower plasma concentrations for propofol when administered in a lipid-free formulation in rats, compared to Diprivan ${ }^{\circledR}$. The lipid-free formulation was designed to simulate the aqueous phase of Diprivan ${ }^{\circledR}$; propofol was dissolved in ethanol (1:5), then mixed with glycerol and dextrose solutions during the infusion. A subsequent study by the same authors demonstrated increased lung concentrations of propofol when administered in the lipid-free formulation (Dutta and Ebling, 1998b). Lung AUC was increased by 300-fold after dosing in the lipid-free formulation, compared to the emulsion. Although the lipid-free formulation was administered at double the propofol dose of the emulsion, this is still a clear effect. Bettschart-Wolfensberger et al. (2000) compared the pharmacokinetics of propofol as an emulsion formulation (Disoprivan $^{\circledR}$ ) or in Tween 80 in goats. The Tween formulation gave significant increases in $t_{1 / 2 \beta}(2.3$-fold $)$ and $A \cup C_{0-\infty}(2.8$-fold $)$, and a $58 \%$ reduction in $\mathrm{CL} ; \mathrm{V}_{\mathrm{ss}}$ was also reduced by $50 \%$, but this was not statistically significant.

Kim et al. (2002) compared the oral and i.v. pharmacokinetics of cyclosporin $A$ in an oil in water emulsion (consisting of $10 \%$ soybean oil and $2 \%$ lecithin) with the commercially available products for each route. No differences in $A U C, C L$, or $t_{1 / 2}$ were 
observed when the emulsion was administered i.v., compared to $\mathrm{CIPOL}^{\circledR}$ injection, although MRT was reduced by $32 \%$. After oral administration, the emulsion gave a $49 \%$ lower $C_{\max }$ and $27 \%$ lower AUC than Sandimmun Neoral ${ }^{\circledR}$ (containing DL- $\alpha$-tocopherol, ethanol, PG, corn oil mono-di-triglycerides and Cremophor RH40), but there were no differences in $t_{1 / 2}, t_{\max }, M R T$, or $F$.

Park et al. (1999) compared the pharmacokinetics of flurbiprofen in aqueous solution with a microemulsion consisting of ethyl oleate, lecithin and DSPE-PEG (8:3:1). The microemulsion gave a $49 \%$ reduction in $C L$, and increased $A U C, M R T$ and $t_{1 / 2}$, by $1.9-, 1.6-$ and 1.5-fold, respectively, with no change in $V_{s s}$. Tissue distribution was also affected by the microemulsion; tissue:plasma ratios for the liver, kidney, lung, spleen and heart were significantly reduced.

Kurihara et al. (1996) reported that the pharmacokinetics of palmitoyl rhizoxin were substantially altered by dosing as an emulsion. Dosing as an emulsion substantially increased drug plasma levels and $A \cup C_{0-\infty}$ (443-fold), and reduced $C L$ (720-fold), compared to dosing in a PEG/DMA solution. Smaller changes in these parameters were seen when dosing in the emulsion was compared to administration in a colloidal solution, with a 4.3-fold increase in $\mathrm{AUC}_{0-\infty}$ and 4.5-fold reduction in $\mathrm{CL}$. It was thought likely that drug precipitation would have occurred after dosing in the 
PEG/DMA solution, which may have contributed to the differences seen. However, no statistical analysis of these findings is reported.

Sakaeda and Hirano (1995) investigated the effects of five emulsion formulations, using various combinations of soybean oil, miglyol, egg yolk phosphatides, cholesterol, and egg phosphatidylcholine, on the pharmacokinetics of sudan II in rats. All of the emulsions tested increased sudan II blood levels and $\mathrm{AUC}_{0-10 \mathrm{~min}}$, compared to administration in plasma. Emulsions composed of Miglyol 812 and egg yolk phosphatides with or without cholesterol also significantly increased $A U C_{0-10 m i n}$ compared to a soybean oil and egg yolk phosphatide emulsion. Organ distribution was influenced by all of the emulsions, compared to administration in plasma. Different effects were seen depending on the composition of the emulsions. Emulsions also have the potential to affect renal elimination. Tibell et al. (1993) reported that a soybean oil and egg phospholipid emulsion gave a transient but non-significant $36 \%$ increase in GFR in rats.

Woolfrey et al. (1989) investigated the effects of Miglyol and fatty acid emulsions, stabilised with $12 \%$ Tween 80 , on the oral absorption of propranolol. The Miglyol 812 emulsion gave significantly shorter MAT and terminal $t_{1 / 2}$ than an aqueous solution or $6 \%$ Tween 80 solution (72 and $39 \%$ reductions, respectively, 
compared to aqueous solution). The opposite effect was seen for the fatty acid emulsion (octanoic acid and lauric acid 1:1). MAT and terminal $t_{1 / 2}$ were significantly increased by 2.2 - and 1.5 -fold, respectively, compared to the aqueous solution. This was attributed to inhibition of gastric emptying by the fatty acids. It was suggested that absorption-rate-limited kinetics may be occurring, as the terminal $t_{1 / 2}$ was significantly longer than that seen after i.v. dosing for all formulations except the Miglyol emulsion.

Formulation as an emulsion does appear to affect the pharmacokinetics of drugs, and in some cases different effects are also seen with different emulsions compositions. The wide variety of emulsion compositions that have been studied, as well as the influence of properties such as emulsion particle size, makes interpretation of the effects of particular components difficult.

\subsection{Conclusions}

The literature contains many reports demonstrating the potential of pharmaceutical excipients to substantially affect drug disposition. These effects should be avoided in pre-clinical studies designed to determine the intrinsic pharmacokinetics of a drug, as they could give a false impression of drug pharmacokinetic properties, and 
potentially lead to inappropriate candidate selection. However, the application of information from the literature to formulation development can be hampered by factors such as uncertainty over the relevance of in vitro findings for in vivo studies, and the fact that doses used in in vivo studies are sometimes higher than those encountered in formulations, as well as the lack of comprehensive studies investigating the effects of excipients on individual aspects of drug disposition. More work is therefore required in this area, to investigate the effects of commonly used co-solvents and excipients on various aspects of drug pharmacokinetics in vivo, at doses relevant to pre-cinical formulation. This thesis therefore aims to investigate the effects of some commonly used pharmaceutical excipients on drug disposition. 


\section{Chapter 2:}

Effects of excipients on in vivo metabolism 


\section{Contents}

2.1 Introduction ..................................................... 46

2.2 Methods and Materials........................................ 52

2.2.1 Materials................................................. 52

2.2 .2 Methods..................................................... 53

2.3 Results....................................................... 57

2.3.1 Aminopyrine breath test (APBT) ...................... 57

2.3.2 Erythromycin breath test (EMBT) ........................ 63

2.3.3 NDMA breath test (NDMA BT) ........................... 69

2.3.4 Caffeine breath test (CBT) ............................ 74

2.4 Discussion ................................................... 75

2.4.1 Conclusions .............................................. 87

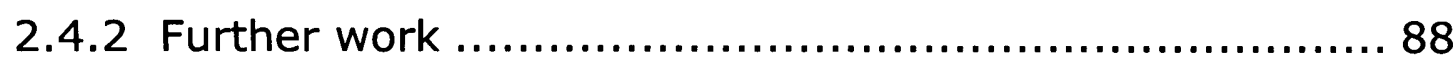




\subsection{Introduction}

As discussed in Chapter 1, a variety of co-solvents and excipients can affect metabolism. Much of the work done to investigate this has been carried out in vitro, using systems such as liver microsomes or purified CYP450 isoforms. However, it is difficult to extrapolate data from these systems to the in vivo situation. Factors such as the concentration of excipient at the site of metabolism cannot be accurately predicted, and the relative contribution of different CYP450 isoforms to total metabolism cannot be readily assessed. Additionally, the influence of complex in vivo factors such as distribution and plasma protein binding cannot be accurately incorporated into in vitro models. Investigations into the effects of co-solvents and excipients on drug metabolism in vivo, at doses commonly used in pre-clinical studies, are therefore needed, in order that the potential effects of these formulation additives on metabolism can be taken into account when designing formulations for early pharmacokinetic studies.

The ${ }^{14} \mathrm{C}$ breath test has been used as a measure of in vivo metabolism in humans and animals. In the breath test, a substrate which is metabolised by demethylation is labelled with ${ }^{14} \mathrm{C}$ at the labile methyl group, and administered to the subject. When the 
substrate undergoes metabolic $\mathrm{N}$-demethylation in vivo, the ${ }^{14} \mathrm{C}$ methyl group is metabolised to formaldehyde, then to formate, and finally to ${ }^{14} \mathrm{CO}_{2}$ which is exhaled in the breath. Using suitable apparatus, the ${ }^{14} \mathrm{CO}_{2}$ exhaled can be collected and quantified by scintillation counting. The ${ }^{14} \mathrm{CO}_{2}$ exhalation data can then be used to calculate a breath elimination half-life for the breath test substrate. It should be noted that, if formation of the demethyl metabolite is the rate-limiting step in its kinetics, the breath test is also capable of reflecting changes in the pharmacokinetics of the parent compound due to factors other than altered metabolism.

A major advantage of the breath test method is that it enables changes in in vivo metabolism to be investigated without the need for any blood sampling. This minimises stress to the animal. It also circumvents operational difficulties in obtaining blood samples of sufficient volume and quality, which could be encountered when attempting to determine plasma pharmacokinetics of the compound of interest. Additionally, the number and volume of blood samples which can be obtained in pharmacokinetic studies are subject to license restrictions; collecting breath is non-invasive and thus not subject to these, and enables the elimination profile to be more fully characterised. A further advantage of the breath test method is that it is only necessary to develop one analysis method for all compounds using scintillation counting, rather than having to 
develop separate methods for each compound, as would be necessary for HPLC analysis.

Various breath test substrates can be used, enabling investigation of the effects of a particular factor on metabolism either by several CYP450 enzymes at once, or by specific isoforms. The aminopyrine breath test (APBT) is generally used as a measure of mixed function oxidase activity or liver metabolic function, as it is metabolised by several CYP450s (Kotake et al., 1982; Tanaka and Breimer, 1997; Van de Casteele et al., 2004; Neef et al., 2006), including CYPs 2C11, 1A, 2A, 2B and 2D1 (Imaoka et al., 1988). Recently, Neef et al. (2006) used the APBT as a measure of liver microsomal function when investigating the effects of the antifibrotic rapamycin in cirrhotic rats. 14 days treatment with rapamycin increased the elimination rate constant for the APBT, demonstrating that the treatment increased aminopyrine clearance, suggesting improved liver metabolic function. Van De Casteele et al. (2004) also used the APBT as a measure of microsomal activity, to investigate the effects of halofuginone on liver fibrosis in bile-duct obstructed rats. They reported a reduction in the APBT elimination rate constant, demonstrating reduced aminopyrine clearance, which suggests that the treatment significantly worsened microsomal function. 
Use of the APBT in combination with other breath tests, to give a broader picture of the effects of a particular factor on metabolism, has been described in several recent publications. Provencher et al. (1999) used the APBT in combination with the erythromycin breath test (EMBT; CYP3A1/2) and caffeine breath test (CBT; CYP1A2) to assess the effect of cyclosporine on recovery of drug metabolism after partial hepatectomy in the rat. The APBT was used as a measure of CYP2B1 and CYP 2C11. Cumulative ${ }^{14} \mathrm{CO}_{2}$ exhalation over 2 hours was reduced for all of the breath tests following partial hepatectomy. The APBT and EMBT were unaffected by cyclosporin treatment. However, cumulative ${ }^{14} \mathrm{CO}_{2}$ exhalation over 2 hours in the CBT was increased $24 \mathrm{~h}$ after hepatectomy by cyclosporin treatment, but not at 48,72 or $96 \mathrm{~h}$ after hepatectomy. Use of several breath test substrates therefore enabled the authors to demonstrate differential effects of cyclosporin treatment on recovery of metabolism by different isoforms of CYP450.

Leblond et al. (2000) used the APBT, EMBT and CBT to investigate the effect of chronic renal failure on hepatic drug metabolism in the rat. Again, use of several breath test substrates enabled differential effects on metabolism by different CYP450 isoforms to be demonstrated. Cumulative ${ }^{14} \mathrm{CO}_{2}$ exhalation over 2 hours was significantly lower in animals with renal failure than in controls for the APBT and EMBT. Correspondingly, expression of CYP2C11 and 
$3 A 1 / 2$ were significantly reduced in these animals, although it should be noted that only levels of these isoforms and CYP1A2 were measured. In contrast, chronic renal failure did not affect the CBT, or expression of CYP1A2, demonstrating that it did not cause a general non-specific reduction in metabolism. However, it should be borne in mind when interpreting these results that the breath test method will also reflect changes in clearance due to factors other than altered metabolism. Renal failure would be expected to increase the half-life of compounds which undergo a substantial percentage of renal elimination. Less than $5 \%$ of caffeine is excreted unchanged in urine (Arnaud, 1976; Walton et al., 2001), and around $6 \%$ of aminopyrine (Lockwood and Houston, 1980). For erythromycin, $27 \%$ of radioactivity is recovered in urine after administration of $\mathrm{N}$-methyl- ${ }^{14} \mathrm{C}$-erythromycin (Lee et al., 1956). It therefore appears unlikely that the changes seen in Leblond et al.'s study are due to a reduction in renal elimination for aminopyrine, although his cannot be ruled out for erythromycin. However, the fact that CYP450 expression correlated with the breath test results suggests that some effects on metabolism had occurred.

Bastien et al. (2000) used the APBT, EMBT and CBT as well as the NDMA breath test (NDMA BT; CYP2E1) to assess changes in CYP450 in different models of cirrhosis. Bile duct ligation reduced cumulative ${ }^{14} \mathrm{CO}_{2}$ exhalation over 2 hours in the APBT, EMBT and CBT, with 
corresponding reductions in CYP2C, CYP3A and CYP1A content. Cumulative ${ }^{14} \mathrm{CO}_{2}$ exhalation in the NDMA BT increased, but there was no significant alteration in CYP2E1 content. In rats with mild $\mathrm{CCl}_{4}$-induced cirrhosis, the only $\mathrm{BT}$ to be significantly affected was the NDMA BT, again showing a significant increase in cumulative ${ }^{14} \mathrm{CO}_{2}$ exhalation; there was also a reduction in $\mathrm{CYP} 2 \mathrm{C}$ expression. In rats with severe $\mathrm{CCl}_{4}$-induced cirrhosis, there were again reductions in cumulative ${ }^{14} \mathrm{CO}_{2}$ exhalation in the APBT, EMBT and CBT, but no significant effect on the NDMA BT, while content of CYP2C, CYP3A, CYP2E1 and CYP1A was reduced. Use of several breath test substrates again provided a more complete picture of metabolic capacity.

This series of experiments aims to use the APBT, EMBT, CBT, and NDMA BT, to investigate the effects of selected excipients on metabolism in vivo. 


\subsection{Methods and Materials}

\subsubsection{Materials}

Dimethylamine- ${ }^{14} \mathrm{C}$-aminopyrine was obtained from Amersham, UK and had a specific activity of $115 \mathrm{mCi} / \mathrm{mmol}$ and a radiochemical purity of $97.9 \%$. ${ }^{14} \mathrm{C}$-erythromycin was obtained from Perkin Elmer, Boston and had a specific activity of $49 \mathrm{mCi} / \mathrm{mmol}$ and a radiochemical purity of greater than $97 \% .1$-methyl- ${ }^{14} \mathrm{C}$-caffeine was obtained from Sigma, UK and had a specific activity of $55 \mathrm{mCi} / \mathrm{mmol}$ and a radiochemical purity of at least $95 \% .{ }^{14} \mathrm{C}$ nitrosodimethlyamine was obtained from American Radiochemicals Inc, St Louis, and had a specific activity of $55 \mathrm{mCi} / \mathrm{mmol}$ and a radiochemical purity of $99 \%$. Methanol, ethanolamine, DMSO, propylene glycol (PG), OptiPhase HiSafe II scintillant and soda lime were obtained from Fisher, UK. Solutol HS15 was a kind gift from AstraZeneca, Alderley Park, UK.

\section{Animals}

Male Wistar rats (220-350g) (Harlan) were group housed on a 12 hour light/dark cycle, with access to water and standard laboratory rodent diet ad libitum. 


\subsubsection{Methods}

\section{Breath tests}

A control breath test was carried out in each rat. After a suitable washout period, the breath test was repeated with administration of excipient prior to the breath test substrate. Excipient doses used are shown in Table 2.1. DMSO and PG were administered i.p. 20 minutes before administration of the breath test substrate. Solutol HS15 was administered i.v. under isoflurane anaesthesia (penile vein) immediately before the breath test substrates. An anaesthetic dose of isoflurane was also used to assist in with i.p. injections where necessary. As isoflurane is less than $0.5 \%$ metabolised (Flecknell and Orr, 2002), it was deemed unlikely to interfere with the breath test results, especially given the low doses administered. Four rats were used to test the effect of each excipient. For the aminopyrine and erythromycin breath tests, each rat was used for one control breath test and one breath test where excipient was administered. For NDMA, each rat was used for one control breath test and all three excipient breath tests, leaving a suitable washout period between each. All of the breath test substrates were administered in saline, with the exception of erythromycin for the $440 \mathrm{mg} / \mathrm{kg}$ DMSO breath test only, which was administered in 50/50 ethanol/saline. This was done as a number of the EMBTs had failed, showing very 
low counts in breath such that the tests had to be repeated. The same formulation was used in the control and treatment breath tests, and resulted in a dose of $0.15 \mathrm{ml}$ ethanol per animal.

Table 2.1: Excipient doses used in the breath tests.

\begin{tabular}{|c|c|c|c|}
\hline Excipient & $\begin{array}{c}\text { Dose } \\
\text { (mg/kg) }\end{array}$ & Formulation & Route \\
\hline DMSO & 220 & $10 \%$ solution, $2 \mathrm{ml} / \mathrm{kg}$ & i.p. \\
DMSO & 440 & $20 \%$ solution, $2 \mathrm{ml} / \mathrm{kg}$ & i.p. \\
DMSO & $3520 *$ & $1 \mathrm{ml}$ neat DMSO & i.p. \\
PG & 829 & $40 \%$ solution, $2 \mathrm{ml} / \mathrm{kg}$ & i.p. \\
Solutol & $90 * *$ & $25 \%$ solution, $0.1 \mathrm{ml}$ & i.v. \\
\hline
\end{tabular}

*For the positive control experiments, $1 \mathrm{ml}$ neat DMSO was administered to each animal, as this was the dose used by Swanson et al. (1981). This corresponded to a dose of $3187-4076 \mathrm{mg} / \mathrm{kg}$. The mean dose, $3520 \mathrm{mg} / \mathrm{kg}$, is used in the text. **Each animal received $0.1 \mathrm{ml}$ of a $25 \%$ solution. This corresponded to a dose of $82-$ $101 \mathrm{mg} / \mathrm{kg}$. The mean dose, $90 \mathrm{mg} / \mathrm{kg}$, is used in the text.

$0.3-1 \mu \mathrm{Ci}$ of the breath test substrate was administered by i.p.

injection. Rats were immediately placed in individual chambers, and air drawn through at rate of approximately $500 \mathrm{ml} / \mathrm{min}$. Air drawn through the chamber was bubbled through $20-25 \mathrm{ml}$ of trapping fluid (3:1 methanol/ethanolamine), then passed through a soda lime trap (Figure 2.1). Efficacy of the methanol/ethanolamine trapping fluid was confirmed using a second test tube of trapping fluid.

Trapping fluid was changed every 10 minutes for the first hour, then every 30 minutes for next 2 hours, then every hour. All breath tests 
were run for 6 hours, except NMDA which was run for 3 hours.

Collected trapping fluid was replenished to its original volume with

methanol, and $1 \mathrm{ml}$ aliquots used for scintillation counting with $2 \mathrm{ml}$

of scintillant. Samples were counted using a Packard TriCarb

2900TR liquid scintillation counter with external standard quench correction.

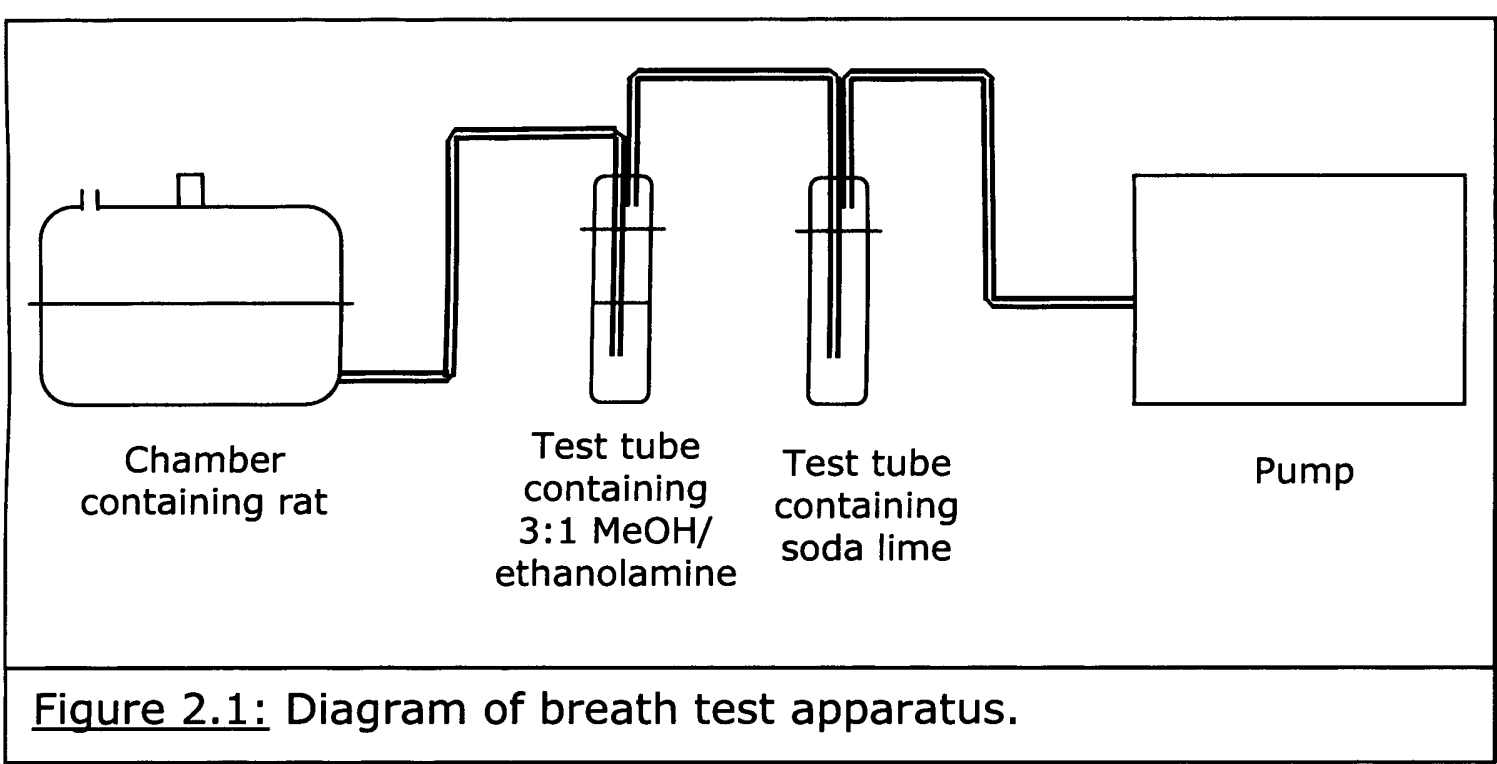

\section{Data analysis}

Background counts were subtracted from the measured counts obtained, and the ${ }^{14} \mathrm{CO}_{2}$-exhalation rate (CER) (\% dose/min) for each time period was calculated with reference to a known aliquot of breath test substrate, counted on the day of the breath test. 
The CER/time curve for each rat was fitted by non-linear regression using Minim version 3.1 (Purves, R.D., University of Otago, New Zealand) to a 1-or 2-compartment model $\left(C=C_{0} \cdot e^{-k t}\right.$ or $\left.C=A e^{-a t}+B e^{-\beta t}\right)$. Rate constants were used to calculate the corresponding $t_{1 / 2}$ (In $2 /$ rate constant). Points before the peak CER was reached were excluded from the curve fitting. For the NDMA BT, it was also sometimes necessary to exclude the last data point from fitting in order to obtain a suitable fit. Peak CER and $t_{\max }$ were read from the plot of CER vs. time. MRT was calculated by dividing AUMC by AUC, both of which were calculated by a log-linear trapezoidal method.

${ }^{14} \mathrm{CO}_{2}$ exhalation half-life, $\mathrm{k}_{1}$, peak CER and MRT were compared between control and treatment breath tests using a one-way ANOVA. A Mann-Whitney test was used to compare $t_{\max }$. For the NDMA BT results, as several treatments were performed in the same animal and therefore compared to the same set of baseline data, a one-way ANOVA with Bonferroni's post-hoc test was used for half-life, $k_{1}$, peak CER and MRT, and homogeneity of variance was assessed using Levene's test. A Kruskal-Wallis test was used to compare $t_{\max }$ for the NDMA BT data. Statistical tests were performed using SPSS (SPSS Inc.). Differences where $p<0.05$ were considered significant. 


\subsection{Results}

\subsubsection{Aminopyrine breath test (APBT)}

An example of the ${ }^{14} \mathrm{CO}_{2}$ exhalation profile for the APBT is shown in Figure 2.2. The exhalation profile exhibits two exponential declines; an initial, fast decline with an average $t_{1 / 2}$ of 23 minutes, and a second, slower, decline with an average $t_{1 / 2}$ of 222 minutes. On average, $59 \%$ of the ${ }^{14} \mathrm{C}$-aminopyrine dose was recovered as ${ }^{14} \mathrm{CO}_{2}$.

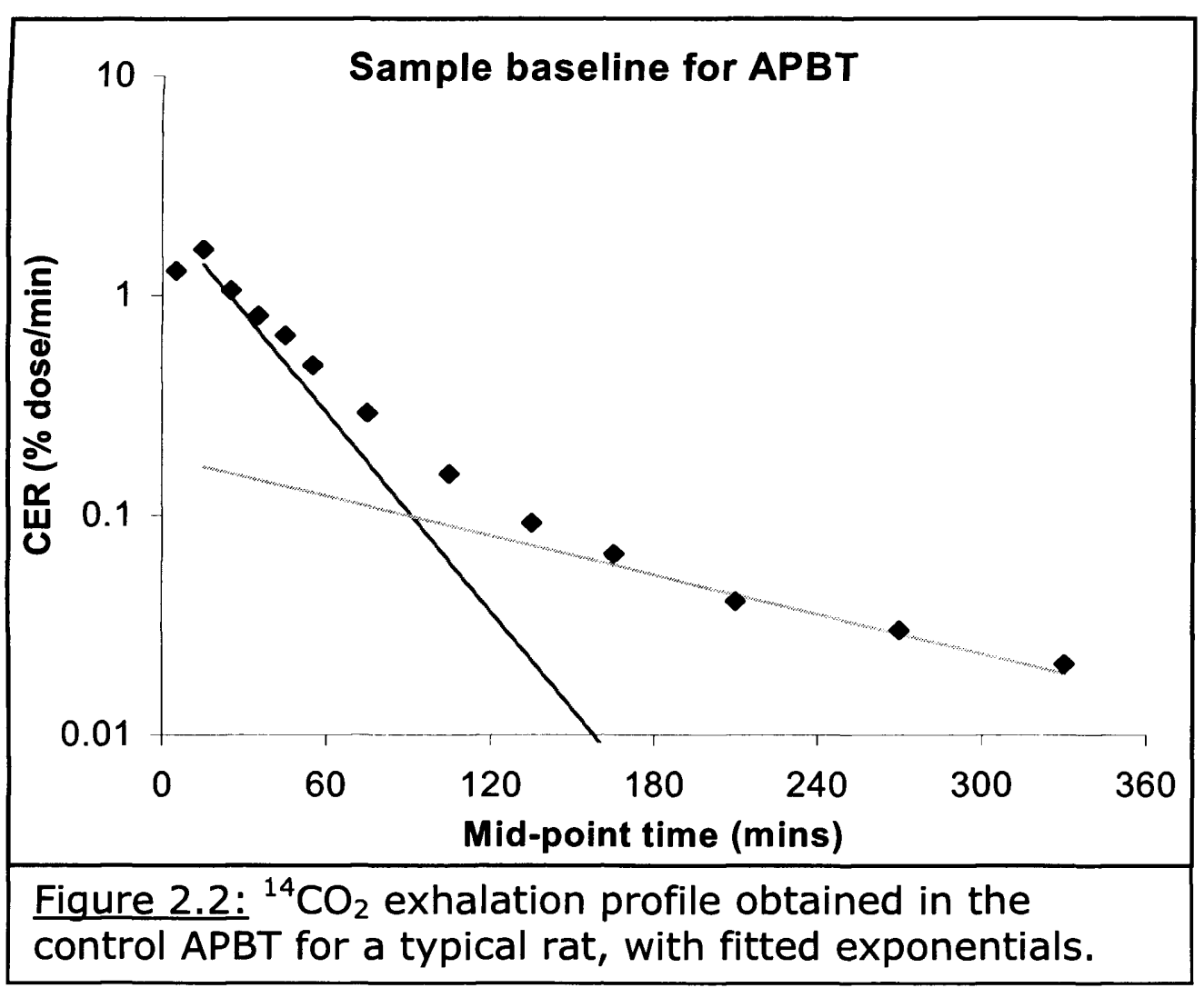




\section{DMSO}

The effects of $3520 \mathrm{mg} / \mathrm{kg}$ DMSO on the APBT parameters are shown in Table 2.2. The CER data for three of the four rats was best fitted by a one compartment model. Pre-treatment with this dose of DMSO resulted in significant increases in half-life, $t_{\max }$ and MRT, and significant reductions in $\mathrm{k}_{1}$ and peak CER. A typical ${ }^{14} \mathrm{CO}_{2}$ exhalation profile is shown in Figure 2.3.

Table 2.2: Effect of $3520 \mathrm{mg} / \mathrm{kg}$ DMSO on key parameters of the APBT.

\begin{tabular}{|c|c|c|c|c|c|c|}
\hline & & Rat 1 & Rat 2 & Rat : & Rat & Mean ( \pm SD) \\
\hline & retrl & .038 & 0.035 & 0.044 & 0.058 & $0.044( \pm 0.010)$ \\
\hline & Test $^{a}$ & .015 & 0.007 & 0.020 & 0.010 & $0.013 *( \pm 0.005)$ \\
\hline & & .07 & 20.02 & 5.72 & 11.97 & $16.44( \pm 3$. \\
\hline & & & & & 64 & $61.31 *( \pm 25.74)$ \\
\hline & Ctrl & & 1.63 & 1.68 & 2.02 & $1.72( \pm 0.19)$ \\
\hline & Test & 68 & 0.29 & 0.34 & 0.27 & $( \pm 0.19)$ \\
\hline & & & & & & $( \pm 0.0)$ \\
\hline & Test & 25.0 & 105.0 & 105.0 & 75.0 & $( \pm 37.7)$ \\
\hline & & & 72.2 & 70.0 & 81.3 & $( \pm 5.8)$ \\
\hline & Test & 84.4 & 184.8 & 191.0 & 165.3 & $156.4 *( \pm 49.2)$ \\
\hline
\end{tabular}

*significantly different from control $p<0.05$. $a$-as the test data were fitted using a one compartment model, $k_{1}$ and $t_{1 / 2 \alpha}$ are in fact $k$ and $t_{1 / 2}$. 


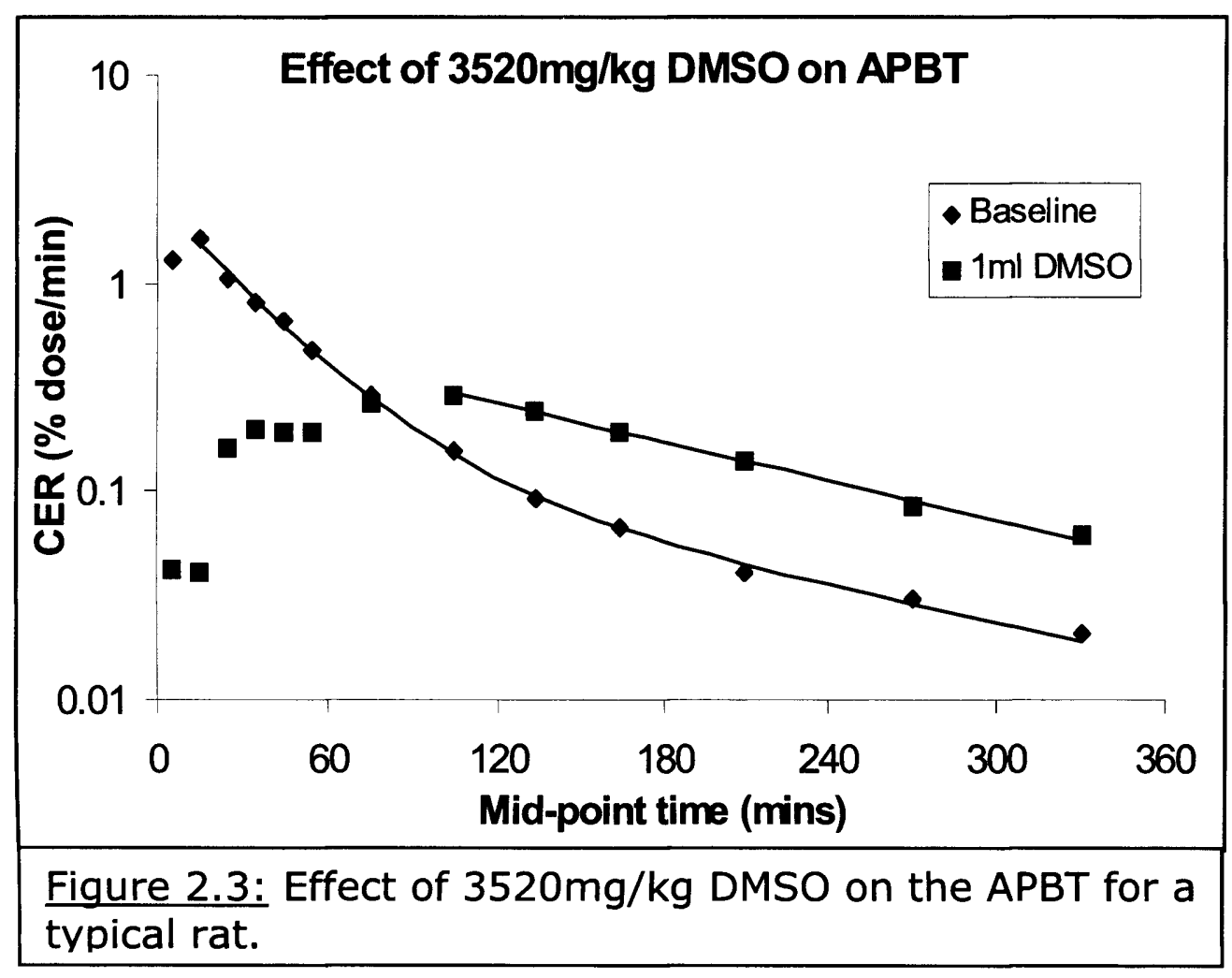


The effects of $220 \mathrm{mg} / \mathrm{kg}$ DMSO on the breath test parameters are shown in Table 2.3. There were no statistically significant differences in any of the breath test parameters between the control and excipient treatment groups.

Table 2.3: Effect of $220 \mathrm{mg} / \mathrm{kg}$ DMSO on key parameters of the APBT.

\begin{tabular}{|c|c|c|c|c|c|c|}
\hline & & Rat 1 & Rat 2 & Rat 3 & Rat 4 & Mean ( \pm SD) \\
\hline \multirow{2}{*}{$\begin{array}{c}\mathbf{k}_{\mathbf{1}} \\
\left(\mathrm{min}^{-1}\right)\end{array}$} & Ctrl & 0.023 & 0.024 & 0.053 & 0.033 & $0.033( \pm 0.014)$ \\
\hline & Test & 0.023 & 0.038 & 0.036 & 0.044 & $0.035( \pm 0.009)$ \\
\hline \multirow{2}{*}{$\begin{array}{l}\mathbf{t}_{1 / 2 \alpha} \\
(\min )\end{array}$} & Ctrl & 30.27 & 28.48 & 13.12 & 21.25 & $23.28 \quad( \pm 7.81)$ \\
\hline & Test & 30.27 & 18.12 & 19.05 & 15.80 & $20.81 \quad( \pm 6.45)$ \\
\hline \multirow{2}{*}{$\begin{array}{c}\text { Peak CER } \\
\text { (\%dose/min) }\end{array}$} & Ctrl & 1.01 & 0.68 & 0.77 & 1.30 & $0.94 \quad( \pm 0.28)$ \\
\hline & Test & 1.03 & 0.93 & 1.16 & 1.43 & $( \pm 0.22)$ \\
\hline \multirow{2}{*}{$\begin{array}{l}\mathbf{t}_{\max } \\
(\min )\end{array}$} & Ctrl & 15.0 & 25.0 & 15.0 & 15.0 & $( \pm 5.0)$ \\
\hline & Test & 15.0 & 25.0 & 15.0 & 15.0 & $( \pm 5.0)$ \\
\hline \multirow{2}{*}{$\begin{array}{l}\text { MRT } \\
(\min )\end{array}$} & Ctrl & 283.1 & 103.7 & 104.0 & 72.7 & $140.9 \quad( \pm 95.9)$ \\
\hline & Test & 284.2 & 80.2 & 70.3 & 66.0 & $125.2( \pm 106.2)$ \\
\hline
\end{tabular}

The effects of $440 \mathrm{mg} / \mathrm{kg}$ DMSO on the APBT are shown in Table 2.4. There were no statistically significant differences in any of the breath test parameters between the control and excipient treatment groups. 
Table 2.4: Effect of $440 \mathrm{mg} / \mathrm{kg}$ DMSO on key parameters of the APBT.

\begin{tabular}{|c|c|c|c|c|c|c|}
\hline & & Rat 1 & Rat : & Rat & Rat 4 & Mean ( \pm SD) \\
\hline r & Ctrl & 0.028 & 0.026 & 0.034 & 0.025 & $0.028( \pm 0.004)$ \\
\hline$\left(\min ^{-1}\right)$ & Test & 0.041 & 0.029 & 0.030 & 0.032 & $0.033( \pm 0.006)$ \\
\hline & Ctrl & 24.72 & 26.56 & 20.62 & 27.34 & $( \pm 3.00)$ \\
\hline & Test & 16.74 & 24.07 & 22.95 & 21.36 & 21.28 \\
\hline & Ctrl & 1.01 & 0.95 & 0.87 & 0.86 & $( \pm 0.07)$ \\
\hline & Test & 1.11 & 0.87 & 0.88 & 0.96 & $( \pm 0.11)$ \\
\hline & Ctrl & & 15.0 & & 15.0 & $( \pm 0.0)$ \\
\hline & Test & 15.0 & 15.0 & 15.0 & 15.0 & $( \pm 0.0)$ \\
\hline & Ctr & 98.7 & 154.9 & 93.8 & 346.0 & $173.4( \pm 118.4)$ \\
\hline & Test & 74.3 & 93.0 & 86.2 & 88.3 & $85.4 \quad( \pm 8.0)$ \\
\hline
\end{tabular}

\section{Propylene glycol}

The effects of $829 \mathrm{mg} / \mathrm{kg}$ PG on APBT parameters are shown in Table 2.5. There were no statistically significant differences in any of the breath test parameters between the control and excipient treatment groups.

Table 2.5: Effect of $829 \mathrm{mg} / \mathrm{kg}$ PG on key parameters of the APBT.

\begin{tabular}{|c|c|c|c|c|c|c|c|}
\hline & & Rat 1 & Rat 2 & Rat 3 & Rat 4 & Mea & $( \pm$ SD $)$ \\
\hline s & Ctrl & 0.032 & 0.037 & 0.030 & 0.034 & 0.033 & $( \pm 0.003)$ \\
\hline$\left(\min ^{-1}\right)$ & Test & 0.028 & 0.032 & 0.028 & 0.033 & 0.030 & $( \pm 0.003)$ \\
\hline & Ctrl & 21.68 & 18.58 & 23.14 & 20.43 & 20.95 & $( \pm 1.93)$ \\
\hline$(\mathrm{mi}$ & Test & 24.85 & 21.78 & 24.34 & 20.85 & 22.95 & $( \pm 1.94)$ \\
\hline 1500 & Ctrl & 1.05 & 1.36 & 0.96 & 1.01 & 1.10 & $( \pm 0.18)$ \\
\hline & Test & 1.03 & 1.13 & 0.95 & 0.92 & 1.01 & $( \pm 0.09)$ \\
\hline & Ctrl & 15.0 & 15.0 & 15.0 & 15.0 & 15.0 & $( \pm 0.0)$ \\
\hline & Test & 15.0 & 15.0 & 15.0 & 15.0 & 15.0 & $( \pm 0.0)$ \\
\hline & Ctr & 78.7 & 59.9 & 72.8 & 75.3 & 71.7 & $( \pm 8.2)$ \\
\hline & Test & 85.4 & 67.5 & 84.7 & 83.4 & 80.3 & $( \pm 8.6)$ \\
\hline
\end{tabular}




\section{Solutol HS15}

Table 2.6 shows the effects of $90 \mathrm{mg} / \mathrm{kg}$ Solutol HS15 on the APBT.

There were no statistically significant differences in any of the breath test parameters between the control and excipient treatment groups. All data were fitted to a two-compartment model, except the control data from rat 4 and test data from rat 2 , which could only be fitted using a one-compartment model.

Table 2.6: Effect of $90 \mathrm{mg} / \mathrm{kg}$ Solutol HS15 on key parameters of the APBT.

\begin{tabular}{|c|c|c|c|c|c|c|}
\hline & & Rat 1 & Rat $2^{a}$ & Rat 3 & Rat $4^{a}$ & Mean ( \pm SD) \\
\hline & Ctrl & 0.025 & 0.024 & 0.025 & 0.020 & $0.024( \pm 0.003)$ \\
\hline$\left(\min ^{-1}\right)$ & Test & 0.029 & 0.019 & 0.030 & 0.022 & $0.025( \pm 0.005)$ \\
\hline$t_{1 / 2 \alpha}$ & Ctrl & 27.28 & 28.64 & 27.49 & 34.96 & $( \pm 3.63)$ \\
\hline$(\min )$ & Test & 24.11 & 36.67 & 23.01 & 30.92 & $28.68 \quad( \pm 6.38)$ \\
\hline Peak CER & Ctrl & 0.88 & 0.76 & 0.88 & 0.73 & $( \pm 0.08)$ \\
\hline & Test & 0.99 & 0.88 & 1.08 & 0.51 & $( \pm 0.25)$ \\
\hline$t_{\max }$ & Ctrl & 15.0 & 15.0 & 15.0 & 15.0 & $( \pm 0.0)$ \\
\hline$(\min )$ & Test & 15.0 & 25.0 & 15.0 & 15.0 & $( \pm 5.0)$ \\
\hline MRT & Ctrl & 168.1 & 103.1 & 205.4 & 68.9 & $136.4 \quad( \pm 61.7)$ \\
\hline & Test & 83.4 & 70.6 & 87.0 & 481.6 & $180.6( \pm 200.7)$ \\
\hline
\end{tabular}

$a$-as the control data for rat 2 and the test data for Rat 4 were fitted using a one compartment model, $\mathrm{k}_{1}$ and $\mathrm{t}_{1 / 2 \alpha}$ in these cases are in fact $\mathrm{k}$ and $\mathrm{t}_{1 / 2}$. 


\subsubsection{Erythromycin breath test (EMBT)}

Figure 2.4 shows an example the CER profile obtained for the EMBT. For the majority of rats, the exhalation profile in the control EMBTs exhibited two exponential phases, with average $t_{1 / 2} s$ of 30 and 205 minutes, for the initial and second exponential declines, respectively. However, the control data for one rat in the PG experiment could only be fitted by a one-compartment model. Overall, variability in the EMBT appeared to be greater than for the APBT and NMDA BT, as demonstrated by the large standard deviations for the control data. Average recovery of ${ }^{14} \mathrm{CO}_{2}$ for the control breath tests was $20 \%$.

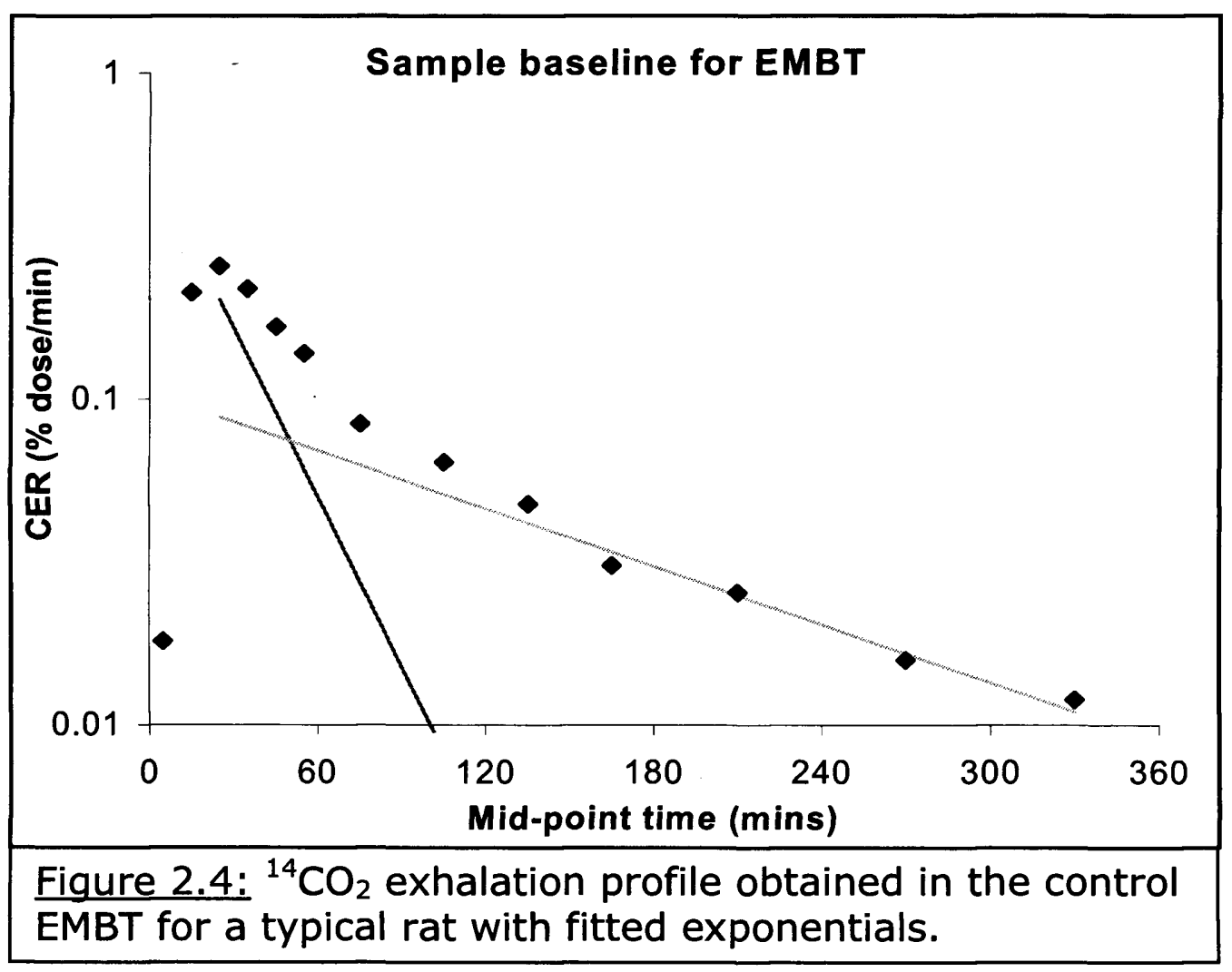




\section{DMSO}

The effects of $3520 \mathrm{mg} / \mathrm{kg}$ DMSO on the EMBT are shown in Table 2.7. As seen for the APBT, this dose of DMSO again changed the exhalation profile from a two compartment to a one compartment model. Half-life was significantly increased, and $k_{1}$ significantly decreased. A typical ${ }^{14} \mathrm{CO}_{2}$ exhalation profile is shown in Figure 2.5.

Table 2.7: Effect of $3520 \mathrm{mg} / \mathrm{kg}$ DMSO on key parameters of the EMBT.

\begin{tabular}{|c|c|c|c|c|c|c|}
\hline & Rat 1 & Rat 2 & Rat 3 & Rat 4 & Mean ( \pm SD) \\
\hline \multirow{2}{*}{$\begin{array}{c}\mathbf{k}_{\mathbf{1}} \\
\left(\mathrm{min}^{-1}\right)\end{array}$} & Ctrl & 0.021 & 0.026 & 0.027 & 0.064 & $0.035( \pm 0.020)$ \\
\hline & Test ${ }^{a}$ & 0.007 & 0.009 & 0.007 & 0.011 & $0.009 *( \pm 0.002)$ \\
\hline \multirow{2}{*}{$\begin{array}{l}\mathbf{t}_{1 / 2 \alpha} \\
(\min )\end{array}$} & Ctrl & 32.65 & 26.53 & 25.65 & 10.77 & $23.90 \quad( \pm 9.28)$ \\
\hline & Test $^{a}$ & 95.71 & 75.49 & 103.84 & 63.45 & $84.62 *( \pm 18.47)$ \\
\hline \multirow{2}{*}{$\begin{array}{c}\text { Peak CER } \\
\text { (\%dose/min) }\end{array}$} & Ctrl & 0.25 & 0.26 & 0.23 & 0.18 & $0.23 \quad( \pm 0.04)$ \\
\hline & Test & 0.15 & 0.26 & 0.16 & 0.21 & $( \pm 0.05)$ \\
\hline \multirow{2}{*}{$\begin{array}{c}t_{\max } \\
(\min )\end{array}$} & Ctrl & 25.0 & 25.0 & 25.0 & 35.0 & $( \pm 5.0)$ \\
\hline & Test & 35.0 & 25.0 & 25.0 & 25.0 & $( \pm 5.0)$ \\
\hline \multirow{2}{*}{$\begin{array}{l}\text { MRT } \\
(\min )\end{array}$} & Ctrl & 138.6 & 131.5 & 110.8 & 165.3 & $( \pm 22.5)$ \\
\hline & Test & 166.1 & 128.7 & 171.9 & 125.6 & $( \pm 24.3)$ \\
\hline
\end{tabular}

*significantly different from control $p<0.05$. $a$-as the test data were fitted using a one compartment model, $k_{1}$ and $t_{1 / 2 \alpha}$ are in fact $k$ and $t_{1 / 2}$. 


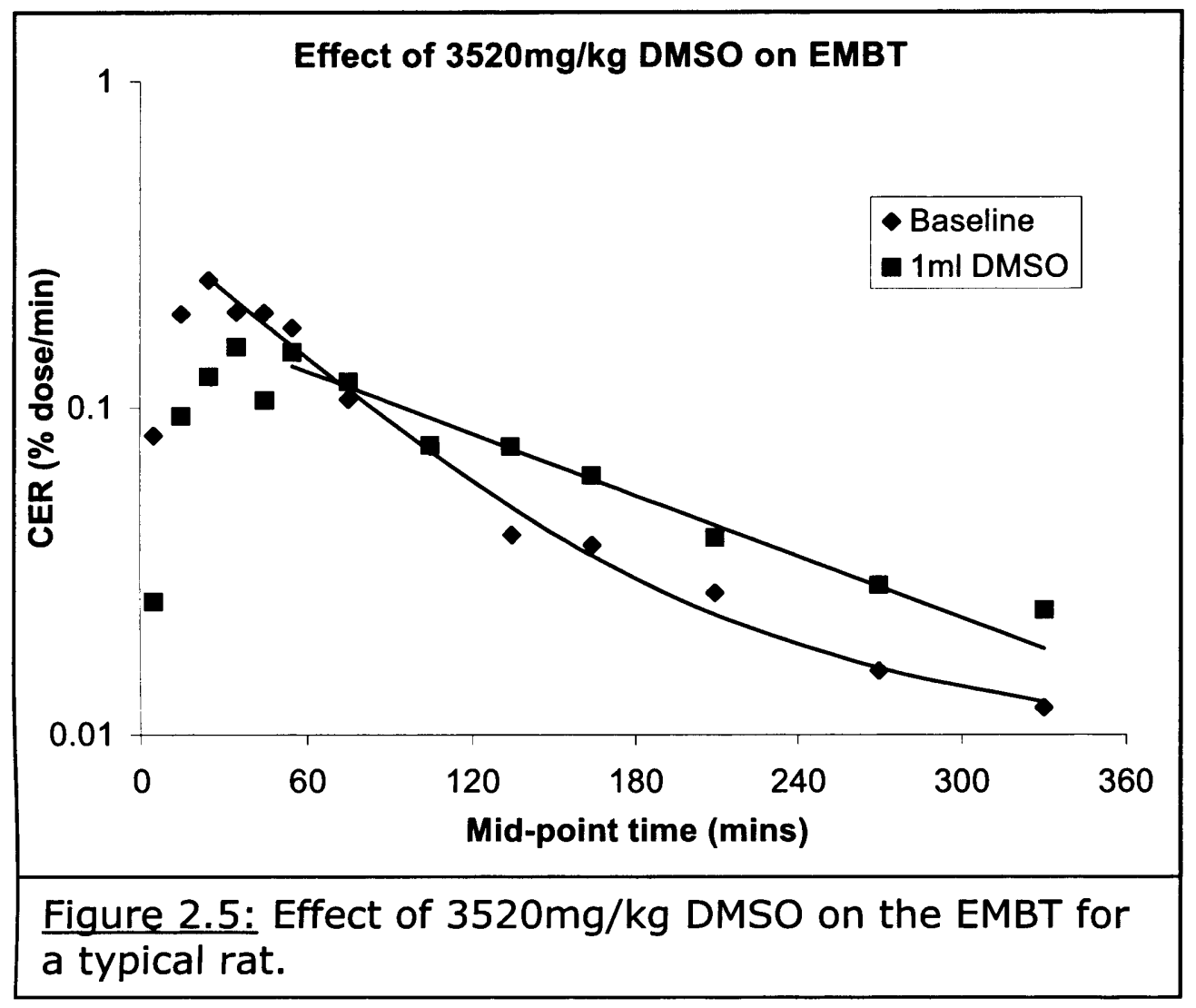


Table 2.8 shows the effects of $220 \mathrm{mg} / \mathrm{kg}$ DMSO on the EMBT. There were no statistically significant differences in any of the breath test parameters between the control and excipient treatment groups.

Table 2.8: Effect of $220 \mathrm{mg} / \mathrm{kg}$ DMSO on key parameters of the EMBT.

\begin{tabular}{|c|c|c|c|c|c|c|}
\hline & & Rat 1 & Rat 2 & Rat 3 & Rat 4 & Mean ( \pm SD) \\
\hline & Ctrl & 0.041 & 0.045 & 0.023 & 0.023 & $0.033( \pm 0.012)$ \\
\hline$\left(\min ^{-1}\right)$ & Test & 0.022 & 0.031 & 0.032 & 0.039 & $0.031( \pm 0.007)$ \\
\hline & Ctrl & 16.89 & 15.31 & 30.10 & 30.62 & $( \pm 8.26)$ \\
\hline$(\min )$ & Test & 31.94 & 22.50 & 21.99 & 17.96 & $( \pm 5.92)$ \\
\hline n & Ctrl & 0.24 & 0.16 & 0.30 & 0.26 & $( \pm 0.06)$ \\
\hline (\%d & Test & 0.28 & 0.17 & 0.23 & 0.25 & $( \pm 0.05)$ \\
\hline & Ctrl & 25.0 & 25.0 & 15.0 & 15.0 & $( \pm 5.8)$ \\
\hline & Test & 15.0 & 35.0 & 25.0 & 25.0 & $( \pm 8.2)$ \\
\hline & & 108.2 & 163.8 & 114.1 & 105.5 & $( \pm 27.5)$ \\
\hline & Test & 119.8 & 141.6 & 188.1 & 129.9 & $( \pm 30.2)$ \\
\hline
\end{tabular}

The higher dose of $440 \mathrm{mg} / \mathrm{kg}$ DMSO also had no significant effect on any of the breath test parameters in the EMBT (Table 2.9).

Table 2.9: Effect of $440 \mathrm{mg} / \mathrm{kg}$ DMSO on key parameters of the EMBT.

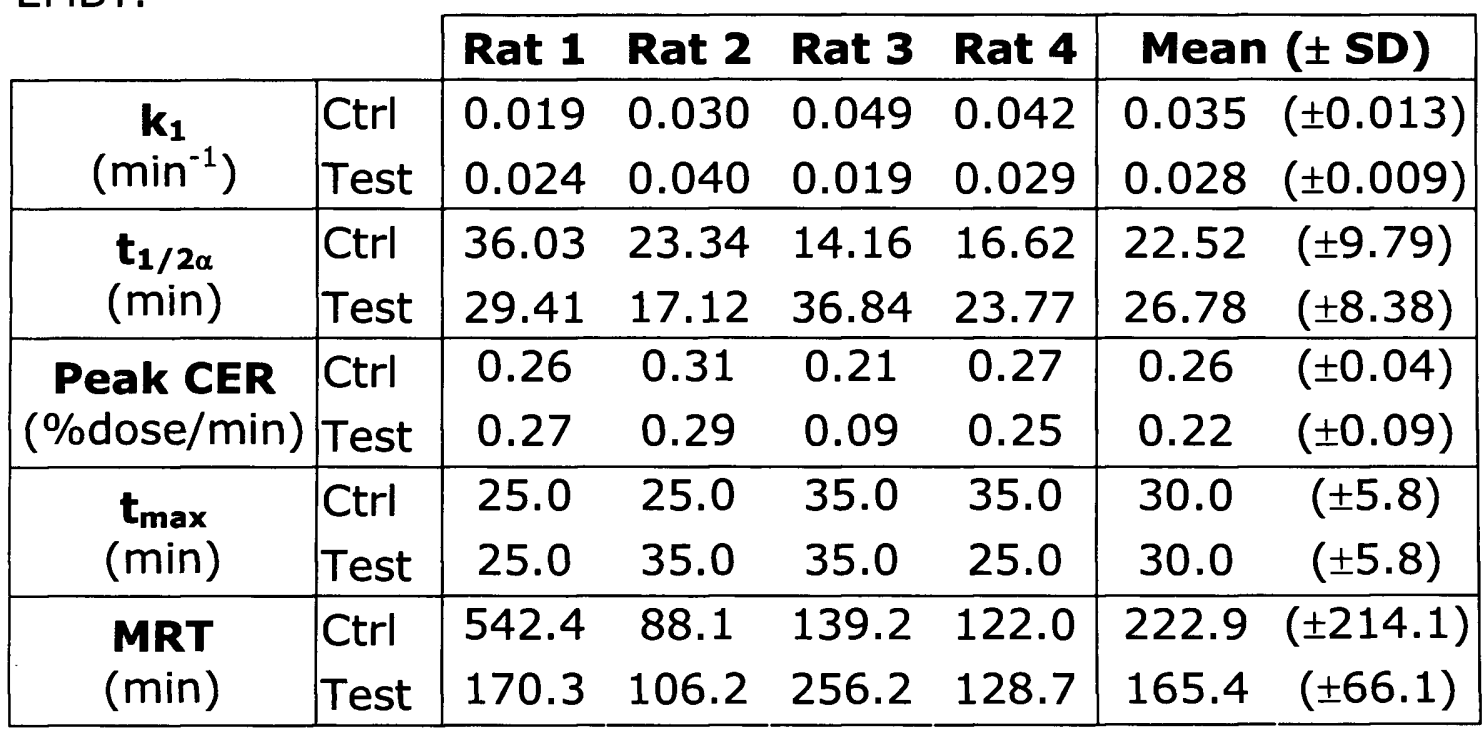




\section{Propylene glycol}

The effects of $829 \mathrm{mg} / \mathrm{mkg}$ PG on the EMBT are shown in Table 2.10.

This excipient had no significant effect on any of the breath test parameters. The data from the control EMBT for rat 3 could only be fitted to a one-compartment model. However, even if this rat is eliminated from the analysis, there are no significant effects on $t_{1 / 2 \alpha}$ or MRT.

Table 2.10: Effect of $829 \mathrm{mg} / \mathrm{kg}$ PG on key parameters of the EMBT.

\begin{tabular}{|c|c|c|c|c|c|c|}
\hline & & Rat 1 & Rat 2 & Rat $3^{a}$ & Rat 4 & Mean ( \pm SD) \\
\hline \multirow{2}{*}{$\begin{array}{c}\mathbf{k}_{\mathbf{1}} \\
\left(\mathrm{min}^{-1}\right)\end{array}$} & Ctrl & 0.036 & 0.024 & 0.010 & 0.040 & $0.028( \pm 0.013)$ \\
\hline & Test & 0.023 & 0.018 & 0.021 & 0.051 & $0.028( \pm 0.015)$ \\
\hline \multirow{2}{*}{$\begin{array}{l}\mathbf{t}_{1 / 2 \alpha} \\
(\mathrm{min})\end{array}$} & Ctrl & 19.38 & 28.58 & 66.89 & 17.42 & $33.07( \pm 23.06)$ \\
\hline & Test & 30.25 & 37.70 & 33.79 & 13.64 & $28.84( \pm 10.58)$ \\
\hline \multirow{2}{*}{$\begin{array}{c}\text { Peak CER } \\
\text { (\%dose/min) }\end{array}$} & Ctrl & 0.15 & 0.24 & 0.19 & 0.26 & $0.21 \quad( \pm 0.05)$ \\
\hline & Test & 0.17 & 0.15 & 0.25 & 0.25 & $( \pm 0.05)$ \\
\hline \multirow{2}{*}{$\begin{array}{l}\mathbf{t}_{\max } \\
(\min )\end{array}$} & Ctrl & 25.0 & 25.0 & 25.0 & 25.0 & $( \pm 0.0)$ \\
\hline & Test & 25.0 & 35.0 & 25.0 & 25.0 & $( \pm 5.0)$ \\
\hline \multirow{2}{*}{$\begin{array}{l}\text { MRT } \\
(\min )\end{array}$} & Ctrl & 126.7 & 152.1 & 114.0 & 120.3 & $( \pm 16.7)$ \\
\hline & Test & 184.5 & 156.1 & 169.8 & 119.8 & $( \pm 27.7)$ \\
\hline
\end{tabular}

$a$-as the control data for rat 3 were fitted using a one compartment model, $\mathrm{k}_{1}$ and $\mathrm{t}_{1 / 2 \alpha}$ are in fact $\mathrm{k}$ and $\mathrm{t}_{1 / 2}$. 


\section{Solutol HS15}

The effects of $90 \mathrm{mg} / \mathrm{kg}$ Solutol HS15 on the EMBT are shown in

Table 2.11. There were no statistically significant differences in any of the breath test parameters between the control and excipient treatment groups.

Table 2.11: Effect of $90 \mathrm{mg} / \mathrm{kg}$ Solutol HS15 on key parameters of the EMBT.

\begin{tabular}{|c|c|c|c|c|c|c|}
\hline & \multirow{2}{*}{$\frac{\text { Rat 1 }}{0.018}$} & \multirow{2}{*}{$\frac{\text { Rat 2 }}{0.015}$} & \multirow{2}{*}{$\frac{\text { Rat } 3}{0.019}$} & \multirow{2}{*}{$\begin{array}{l}\text { Rat 4 } \\
0.011\end{array}$} & Mean ( \pm SD) \\
\hline \multirow{2}{*}{$\underset{\left(\mathrm{min}^{-1}\right)}{\mathbf{k}_{\mathbf{1}}}$} & Ctrl & & & & & $0.016( \pm 0.003)$ \\
\hline & Test & 0.018 & 0.014 & 0.020 & 0.024 & $0.019( \pm 0.004)$ \\
\hline \multirow{2}{*}{$\begin{array}{l}\mathbf{t}_{1 / 2 \alpha} \\
(\mathrm{min})\end{array}$} & Ctrl & 38.02 & 46.85 & 36.93 & 61.80 & $45.90( \pm 11.49)$ \\
\hline & Test & 38.45 & 49.94 & 35.29 & 29.08 & $38.19 \quad( \pm 8.74)$ \\
\hline \multirow{2}{*}{$\begin{array}{c}\text { Peak CER } \\
\text { (\%dose } / \mathrm{min})\end{array}$} & Ctrl & 0.20 & 0.17 & 0.26 & 0.17 & $( \pm 0.04)$ \\
\hline & Test & 0.29 & 0.19 & 0.22 & 0.29 & $( \pm 0.05)$ \\
\hline \multirow{2}{*}{$\begin{array}{c}\mathbf{t}_{\max } \\
(\min )\end{array}$} & Ctrl & 25.0 & 35.0 & 35.0 & 35.0 & $( \pm 5.0)$ \\
\hline & Test & 25.0 & 25.0 & 25.0 & 35.0 & $( \pm 5.0)$ \\
\hline \multirow{2}{*}{$\begin{array}{l}\text { MRT } \\
(\min )\end{array}$} & & 110.5 & 349.8 & 230.4 & 110.7 & $200.4( \pm 114.5)$ \\
\hline & Test & 179.9 & 159.9 & 146.7 & 121.5 & $( \pm 24.5)$ \\
\hline
\end{tabular}




\subsubsection{NDMA breath test (NDMA BT)}

An example of the ${ }^{14} \mathrm{CO}_{2}$ exhalation profile obtained in the NMDA BT is shown in Figure 2.6. The data could only be fitted to a one compartment model; after 3 hours, radioactive counts obtained in the collecting fluid had fallen to background levels, preventing a second compartment from being modelled. Average $t_{1 / 2}$ in the control breath tests was 28 minutes. ${ }^{14} \mathrm{CO}_{2}$ recovery was low, averaging $4.5 \%$ for the control breath tests.

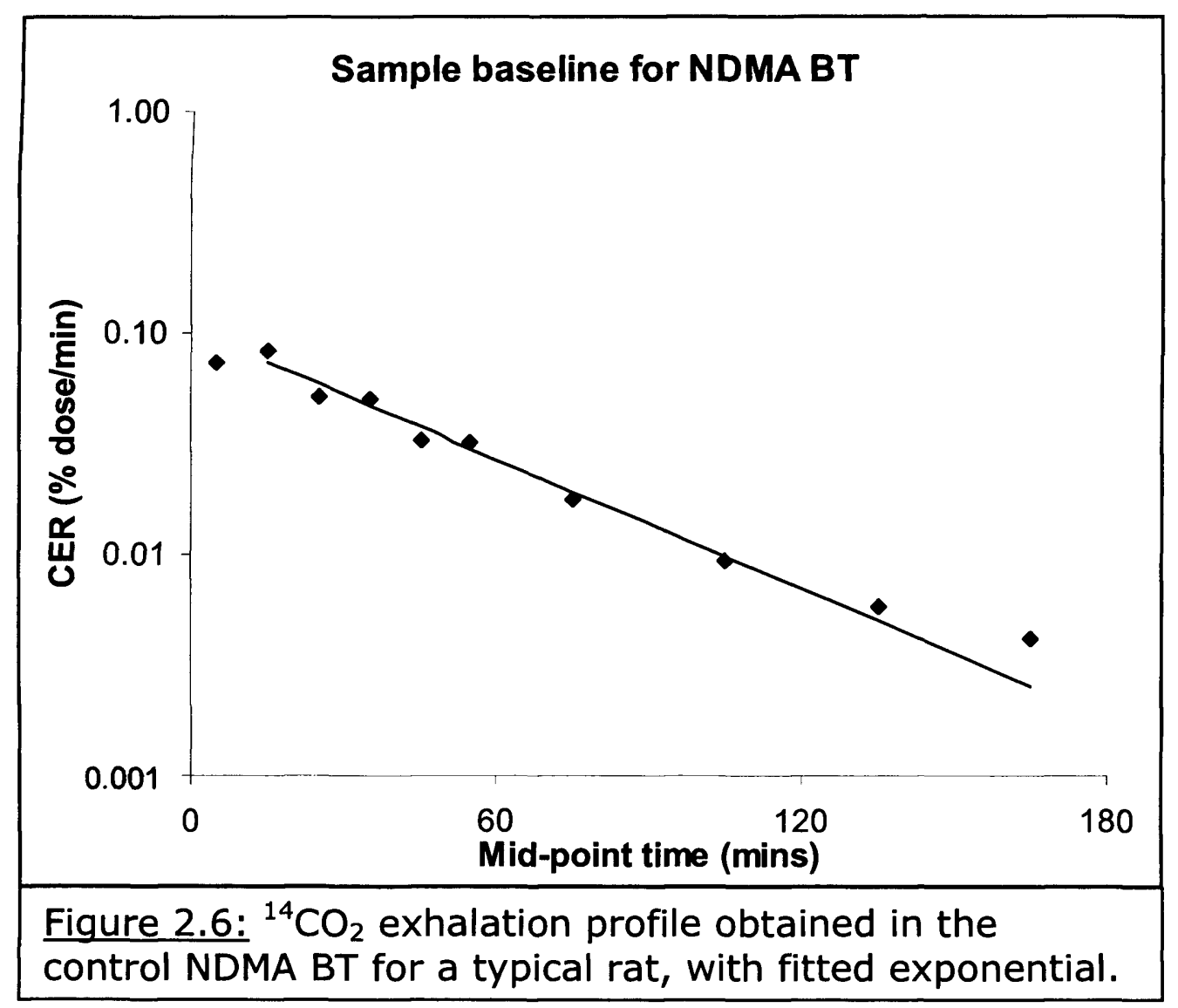




\section{DMSO}

The effects of $3520 \mathrm{mg} / \mathrm{kg}$ on the NDMA BT are shown in Table 2.12 and Figure 2.7. This dose of DMSO significantly increased half-life and MRT, and significantly reduced $k_{1}$ and peak CER.

Table 2.12: Effect of $3520 \mathrm{mg} / \mathrm{kg}$ DMSO on key parameters of the NMDA BT.

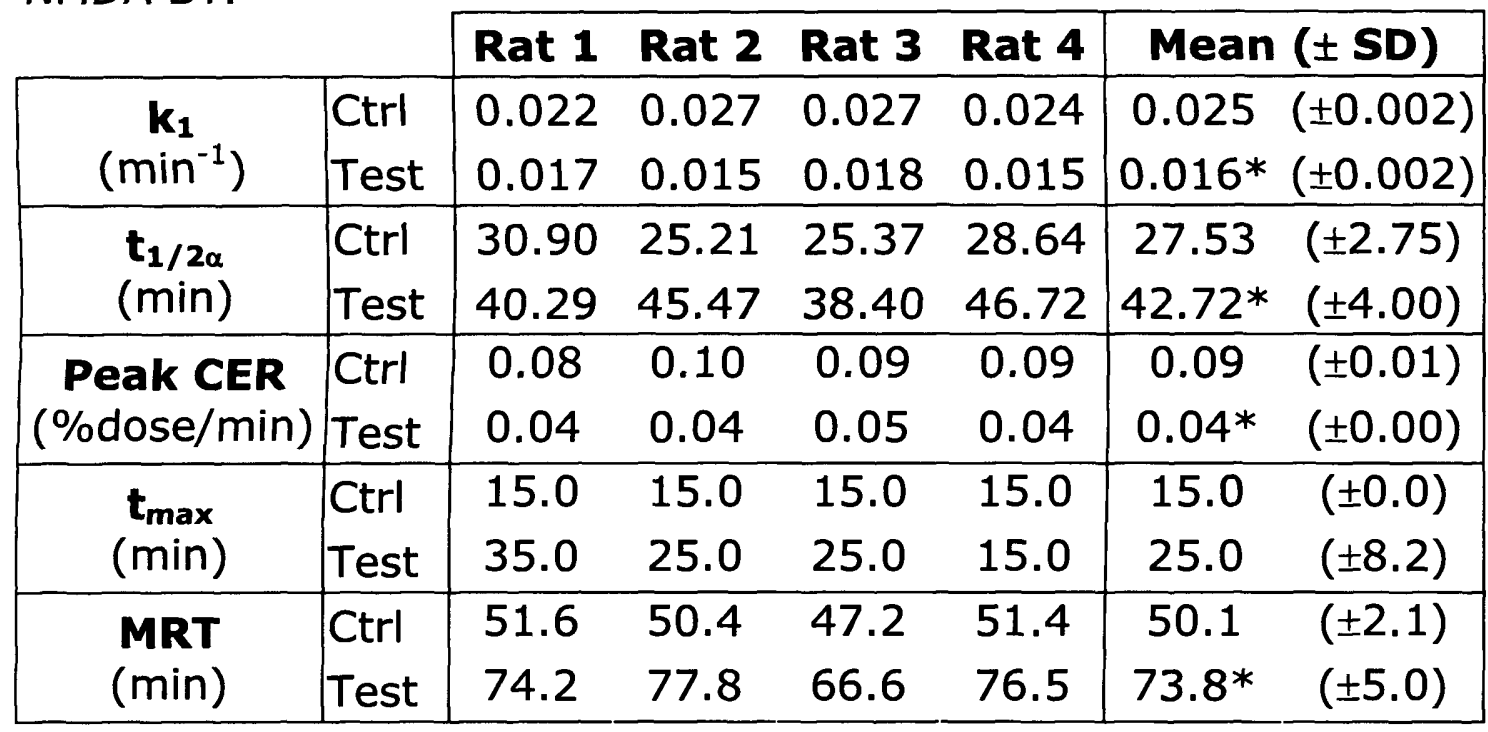

*significantly different from control $p<0.05$. 


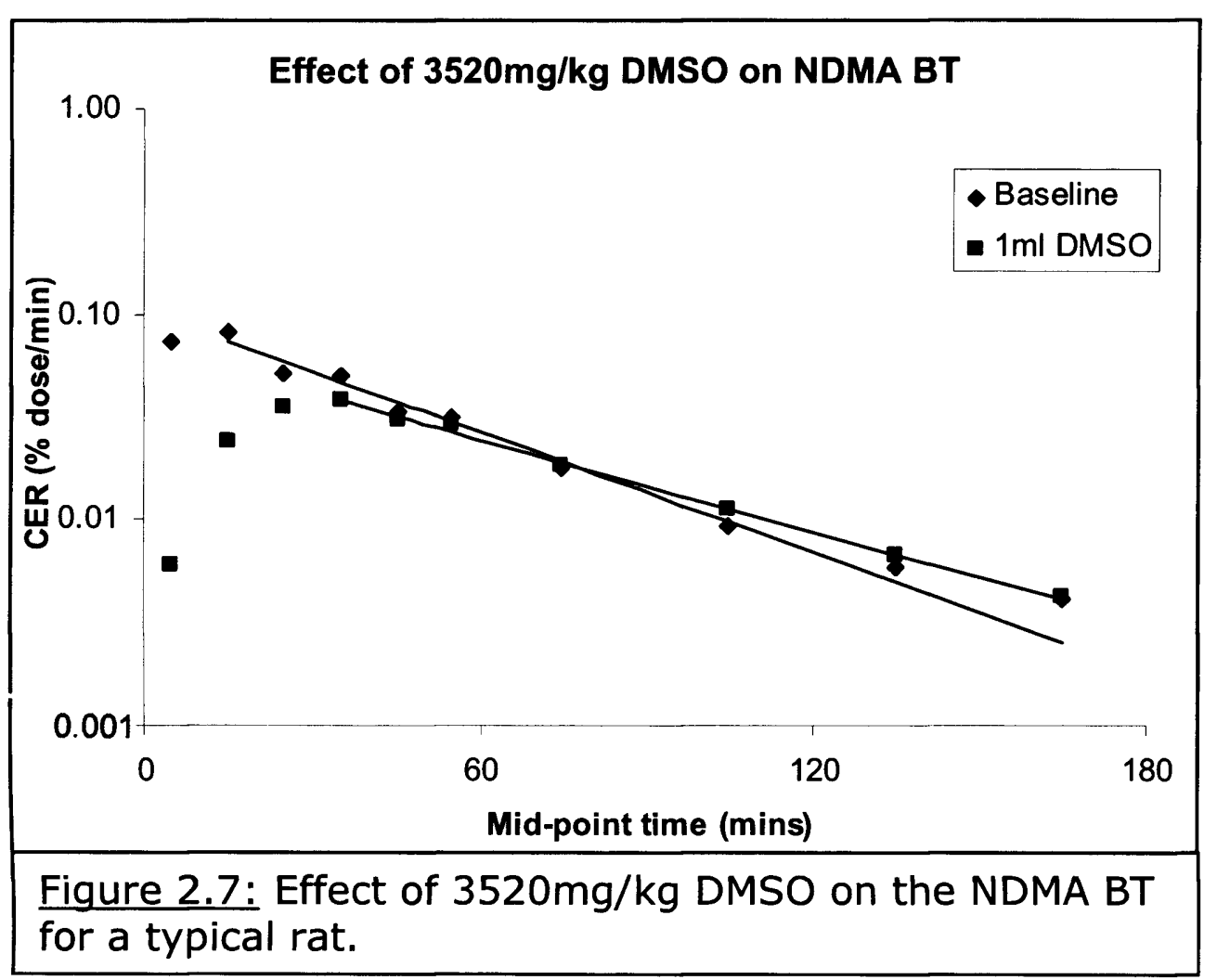


Table 2.13 shows the effects of $220 \mathrm{mg} / \mathrm{kg}$ DMSO on the NDMA BT. This dose of DMSO gave a significant reduction in peak CER, but did not significantly affect any other breath test parameters.

Table 2.13: Effect of $220 \mathrm{mg} / \mathrm{kg}$ DMSO on key parameters of the NMDA BT.

\begin{tabular}{|c|c|c|c|c|c|c|}
\hline & & Rat 1 & Rat 2 & Rat 3 & Rat 4 & Mean ( \pm SD) \\
\hline & Ctrl & 0.022 & 0.027 & 0.027 & 0.024 & $0.025( \pm 0.002)$ \\
\hline$\left(\min ^{-1}\right)$ & Test & 0.020 & 0.020 & 0.026 & 0.020 & $0.021( \pm 0.003)$ \\
\hline & Ctrl & 30.90 & 25.21 & 25.37 & 28.64 & $27.53( \pm 2.75)$ \\
\hline (min) & Test & 34.49 & 35.28 & 26.37 & 35.36 & $( \pm 4.35)$ \\
\hline & Ctrl & 0.08 & 0.10 & 0.09 & 0.09 & $( \pm 0.01)$ \\
\hline (\%d & Test & 0.05 & 0.07 & 0.09 & 0.07 & $0.07 * \quad( \pm 0.02)$ \\
\hline & Ctrl & 15.0 & 15.0 & 15.0 & 15.0 & $15.0 \quad( \pm 0.0)$ \\
\hline$(\min )$ & Test & 15.0 & 15.0 & 15.0 & 15.0 & $( \pm 0.0)$ \\
\hline MRT & Ctrl & 51.6 & 50.4 & 47.2 & 51.4 & $( \pm 2.1)$ \\
\hline$(\min )$ & Test & 62.6 & 63.2 & 52.8 & 59.5 & $( \pm 4.8)$ \\
\hline
\end{tabular}

*significantly different from control $\mathrm{p}<0.05$.

\section{Propylene glycol}

The effects of $829 \mathrm{mg} / \mathrm{kg}$ PG on the NDMA BT are shown in Table 2.14. Pre-treatment with PG significantly increased half-life and MRT, and significantly reduced $\mathrm{k}_{1}$ and peak CER. A typical ${ }^{14} \mathrm{CO}_{2}$ exhalation profile is shown in Figure 2.8 . 
Table 2.14: Effect of $829 \mathrm{mg} / \mathrm{kg}$ PG on key parameters of the NMDA BT.

\begin{tabular}{|c|c|c|c|c|c|c|c|}
\hline & & Rat 1 & Rat 2 & Rat 3 & Rat 4 & Mean & $( \pm$ SD) \\
\hline & Ctrl & 0.022 & 0.027 & 0.027 & 0.024 & 0.025 & $( \pm 0.002)$ \\
\hline$\left(\min ^{-1}\right)$ & Test & 0.017 & 0.015 & 0.018 & 0.015 & $0.016^{*}$ & $( \pm 0.001)$ \\
\hline & Ctrl & 30.90 & 25.21 & 25.37 & 28.64 & 27.53 & $( \pm 2.75)$ \\
\hline (mir & Test & 40.33 & 44.79 & 39.44 & 45.99 & $42.63^{*}$ & $( \pm 3.23)$ \\
\hline K CFR & Ctrl & 0.08 & 0.10 & 0.09 & 0.09 & 0.09 & $( \pm 0.01)$ \\
\hline & Test & 0.06 & 0.06 & 0.05 & 0.06 & $0.06 *$ & $( \pm 0.01)$ \\
\hline & Ctrl & 15.0 & 15.0 & 15.0 & 15.0 & 15.0 & $( \pm 0.0)$ \\
\hline & Test & 15.0 & 25.0 & 25.0 & 15.0 & 20.0 & $( \pm 5.8)$ \\
\hline MRT & Ctrl & 51.6 & 50.4 & 47.2 & 51.4 & 50.1 & $( \pm 2.1)$ \\
\hline & Test & 68.8 & 78.3 & 72.0 & 78.2 & $74.3 *$ & $( \pm 4.7)$ \\
\hline
\end{tabular}

*significantly different from control $\mathrm{p}<0.05$.

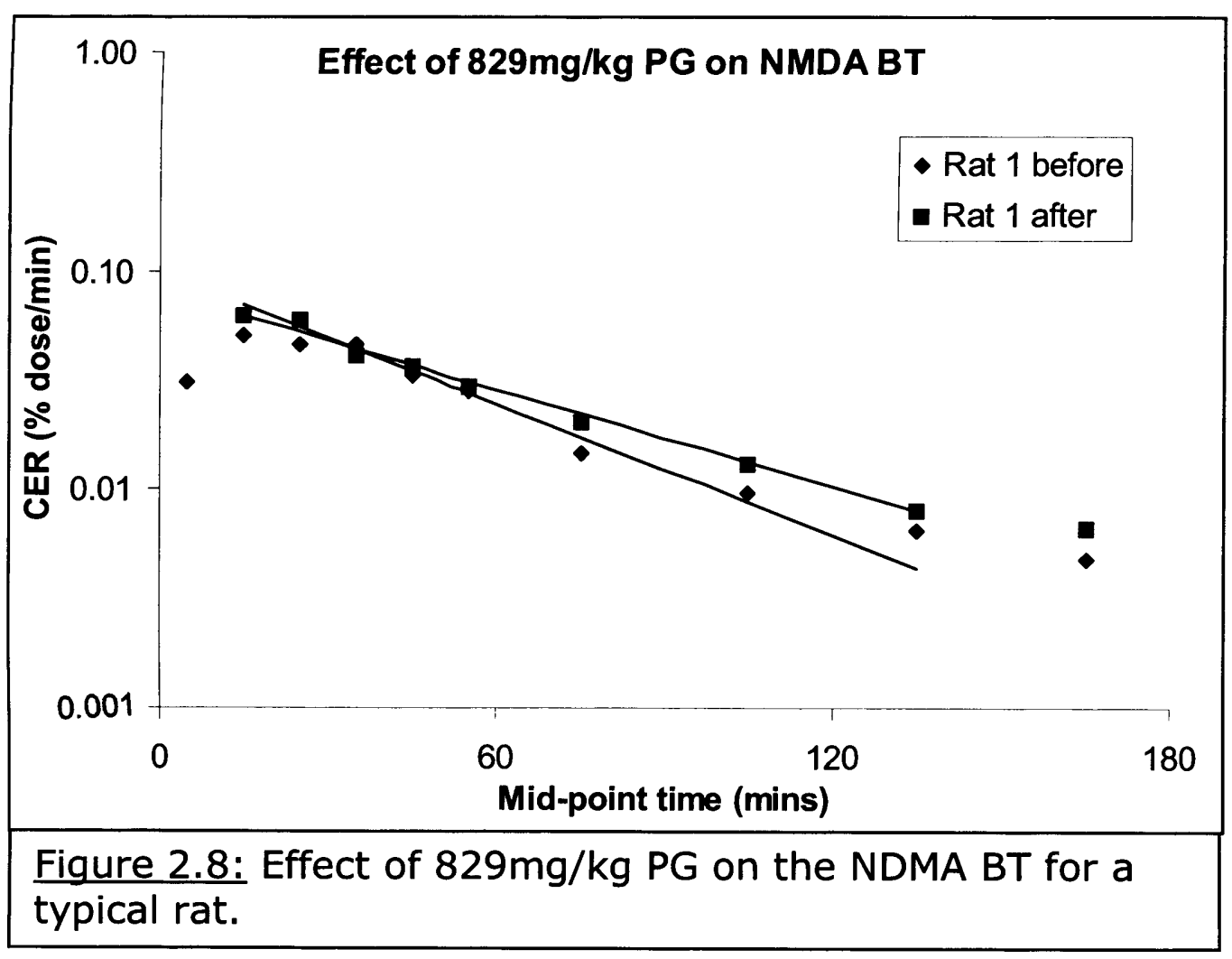




\subsubsection{Caffeine breath test (CBT)}

Figure 2.9 shows the CER profiles obtained for the CBT using 1methyl- ${ }^{14} \mathrm{C}$-caffeine. Average ${ }^{14} \mathrm{CO}_{2}$ recovery was $12 \%$. The profiles obtained were more variable than in the other breath tests (Figure 2.9). The decline in counts was not smooth; after an initial decline, counts rose again in some animals to a second peak, and plateaued before declining again in others. Additionally, counts did not fall very much during the measurement period; even in the profile showing the biggest decline (Rat 4), counts did not fall by one log unit, and the decline was less than this for the other rats. Administration of $3520 \mathrm{mg} / \mathrm{kg}$ DMSO flattened the exhalation profiles, such that very little decline occurred over the 6-hour measurement period (Figure 2.10). Due to license restrictions, it was not possible to keep the animals in the breath test chambers any longer than this. It was decided that the data obtained in the control experiments was unsuitable for use, and so no further breath tests were performed using caffeine. 

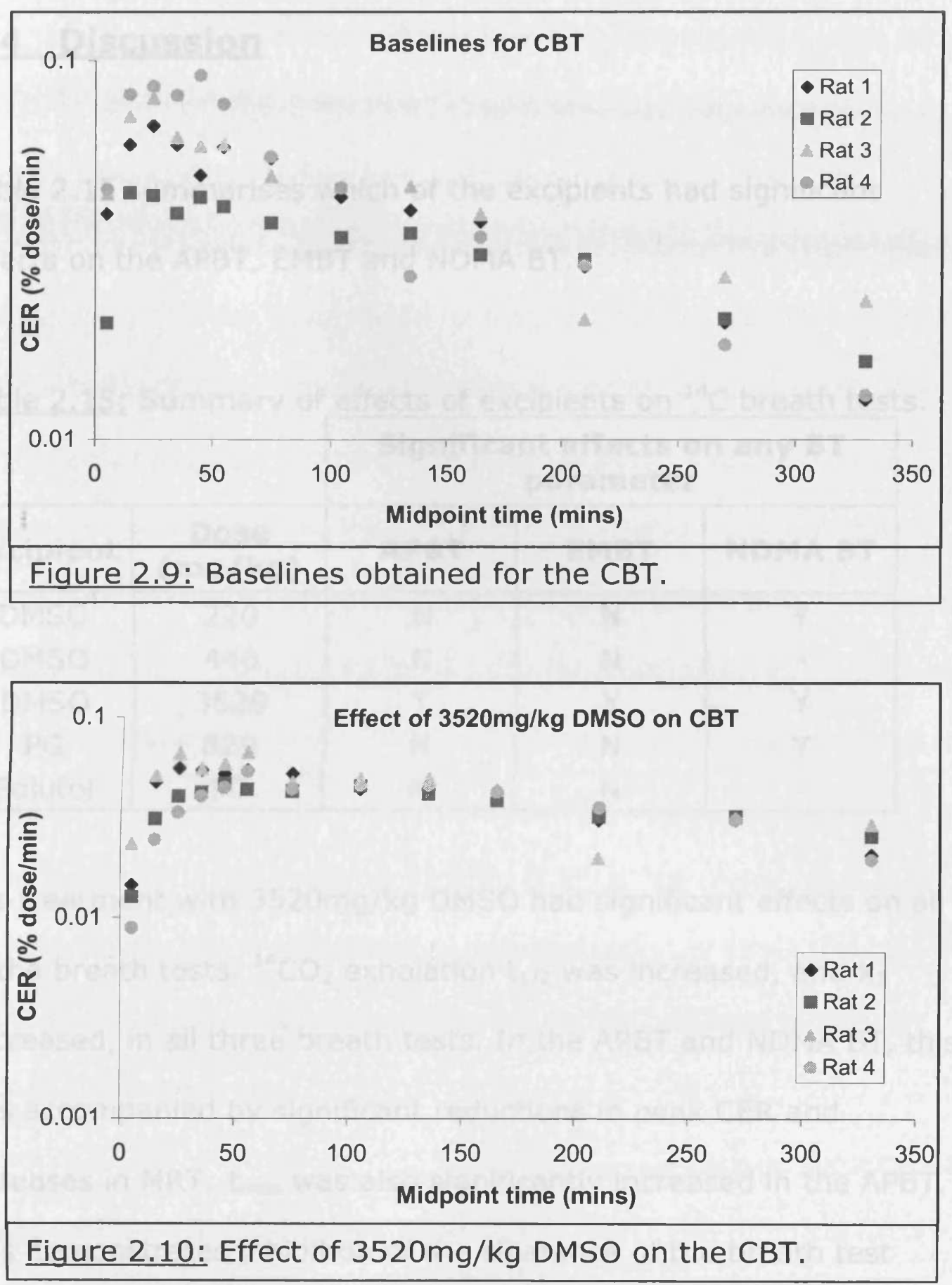


\subsection{Discussion}

Table 2.15 summarises which of the excipients had significant effects on the APBT, EMBT and NDMA BT.

Table 2.15: Summary of effects of excipients on ${ }^{14} \mathrm{C}$ breath tests.

\begin{tabular}{|c|c|c|c|c|}
\cline { 3 - 5 } \multicolumn{2}{c|}{} & \multicolumn{3}{c|}{$\begin{array}{c}\text { Significant effects on any BT } \\
\text { parameter }\end{array}$} \\
\hline Excipient & $\begin{array}{c}\text { Dose } \\
\text { (mg/kg) }\end{array}$ & APBT & EMBT & NDMA BT \\
\hline DMSO & 220 & $\mathrm{~N}$ & $\mathrm{~N}$ & $\mathrm{Y}$ \\
DMSO & 440 & $\mathrm{~N}$ & $\mathrm{~N}$ & - \\
DMSO & 3520 & $\mathrm{Y}$ & $\mathrm{Y}$ & $\mathrm{Y}$ \\
PG & 829 & $\mathrm{~N}$ & $\mathrm{~N}$ & $\mathrm{Y}$ \\
Solutol & 90 & $\mathrm{~N}$ & $\mathrm{~N}$ & - \\
\hline
\end{tabular}

Pre-treatment with $3520 \mathrm{mg} / \mathrm{kg}$ DMSO had significant effects on all of the breath tests. ${ }^{14} \mathrm{CO}_{2}$ exhalation $t_{1 / 2}$ was increased, and $k_{1}$ decreased, in all three breath tests. In the APBT and NDMA BT, this was accompanied by significant reductions in peak CER and increases in MRT; $t_{\max }$ was also significantly increased in the APBT. This demonstrates inhibition of the clearance of the breath test substrates by this dose of DMSO, suggesting inhibition of CYP3A (EMBT), CYP2E1 (NDMA BT) and the variety of enzymes that metabolise aminopyrine (CYP 2C11, 2C12, $2 \mathrm{~B} 1$ and 2B2) (APBT).

In vivo inhibition of metabolism by this dose of DMSO has previously been reported by Swanson et al. (1981), who 
demonstrated a four-fold increase in the $t_{1 / 2}$ of sulindac in rats after administration of $1 \mathrm{ml}$ DMSO i.p. $(3.4-3.6 \mathrm{ml} / \mathrm{kg})$, and reductions in plasma levels of its sulfide and sulfone metabolites. Metabolism of sulindac to the sulfide is catalysed by aldehyde oxidase (Rodrigues, 2005); the enzymes involved in formation of the sulfone do not appear to have been identified. Lower doses of the cosolvent ( 0.1 and $0.5 \mathrm{ml}$ ) reduced plasma levels of the sulfide metabolite, but did not significantly affect sulindac plasma $t_{1 / 2}$ or sulfone metabolite levels. The lack of effect on $t_{1 / 2}$ at these lower doses occurs because sulfide formation is not a route of sulindac elimination (Swanson et al., 1981; Swanson et al., 1983); however this still demonstrates an in vivo effect of the excipient on metabolism. A further study (Swanson et al., 1983) demonstrated that sulfide metabolite formation was also inhibited after administration of DMSO by i.v. infusion and by the p.o. and cutaneous routes, without affecting sulindac plasma levels.

Inhibition of aminopyrine metabolism by DMSO has been reported in vitro at a concentration of $0.7 \mathrm{mmol} / \mathrm{ml}$ in rat liver supernatant (Kawalec and Andrews, 1980). Inhibition of CYP2E1-mediated NDMA demethylation by DMSO has also been reported in vitro, in rat and human liver microsomes, at concentrations of $0.2 \%$ and above (Yoo et al., 1987; Chauret et al., 1998). Inhibition of CYP3A4, corresponding to our EMBT, by DMSO $(0.2 \%)$ in human liver 
microsomes was reported by Chauret et al. (1998). The breath test results with $1 \mathrm{ml}$ DMSO confirm these findings. However, this dose of DMSO would be unlikely to be used as a formulation, and was employed here to act as a positive control and facilitate comparison with the work of Swanson et al. (1981).

It is of interest that no effect on MRT was seen in the EMBT with $3520 \mathrm{mg} / \mathrm{kg}$ of DMSO, despite the large and significant increases in $t_{1 / 2}$. The inhibition of metabolism caused by DMSO changed the elimination profile from biphasic to monophasic, so that the $2^{\text {nd }}$ slower $t_{1 / 2}$ did not become apparent during the experiment. MRT was therefore based on extrapolation of the shallow $2^{\text {nd }}$ elimination phase for the control data, but could only be based on the steeper slope of the single phase in the test animals which, although much shallower than $t_{1 / 2 \alpha}$ in the control animals, was steeper than $t_{1 / 2 \beta}$. MRT therefore did not significantly increase in the EMBT test group. If breath had been collected for longer, the second elimination phase may have become apparent, enabling it to be modelled and more representative MRTs to be derived. However, the amount of time the animals are allowed to stay in the apparatus for is limited. In contrast, the change of the elimination profile from biphasic to monophasic did not prevent a significant increase in MRT in the APBT. This appears to be due to other changes in the shape of the ${ }^{14} \mathrm{CO}_{2}$ exhalation profile which occurred in the APBT; $t_{\max }$ was 
increased, and peak CER reduced, by this dose of DMSO. This, in combination with the increased $t_{1 / 2}$, appears to be responsible for the increase in MRT in the APBT. MRT therefore does not appear to be a sensitive parameter to use in interpretation of breath test data, as it did not change to reflect the inhibitory effects of DMSO in all of the breath tests, despite their being clearly visible upon examination of the ${ }^{14} \mathrm{CO}_{2}$ exhalation profiles.

The lower doses of DMSO (220 and $440 \mathrm{mg} / \mathrm{kg}$ ) typically used in formulations for early pharmacokinetic studies (M. De Matas, personal communication) did not significantly affect any of the breath test parameters in the APBT or EMBT. $220 \mathrm{mg} / \mathrm{kg}$ DMSO did cause a significant reduction in peak CER in the NDMA BT, from 0.093 to $0.068 \%$ of the dose per minute. However, this change is small, and was not accompanied by any significant alteration in ${ }^{14} \mathrm{CO}_{2}$ exhalation $t_{1 / 2}$ or $k_{1}$, suggesting that the clearance of NDMA was not significantly affected by this dose of DMSO. It therefore appears that the lower doses of DMSO do not affect metabolism by CYP3A, CYP2E1, or the variety of enzymes which metabolise aminopyrine (CYP 2C11, 2C12, 2B1 and 2B2), to any significant extent. It may be that, at these doses, concentrations of DMSO in hepatocytes are not sufficient, or the residence time is not sufficiently long, to produce any significant inhibition of metabolism. These results demonstrate that, although DMSO can inhibit 
metabolism in vivo, this does not appear to be an effect after a single administration of the low doses commonly used in pre-clinical studies. However, the significant inhibition seen with the higher dose of DMSO demonstrates that use of excessive doses of this excipient should be avoided. It is also possible that significant effects would occur after repeated administration of the lower doses tested here, for example in chronic dosing studies.

PG is used in commercial products at concentrations ranging from 0.2 to $75 \%$ (Nema et al., 1997), and is commonly used in early pharmacokinetic studies at doses of up to $1036 \mathrm{mg} / \mathrm{kg}(50 \%$ solution, $2 \mathrm{ml} / \mathrm{kg}$ dose volume) (M. De Matas, personal communication), although lower concentrations are usually used. $829 \mathrm{mg} / \mathrm{kg}$ ( $40 \%$ solution, $2 \mathrm{ml} / \mathrm{kg}$ ) was therefore considered a reasonable dose to use in these investigations. In the NDMA BT, pre-treatment with PG gave significant increases in ${ }^{14} \mathrm{CO}_{2}$ exhalation $t_{1 / 2}$ and MRT, and significant reductions in $k_{1}$ and peak CER. This demonstrates a reduction in NDMA clearance, suggesting inhibition of CYP2E1. However, PG did not significantly affect any of the breath test parameters in the APBT or EMBT, suggesting a specific effect on CYP2E1. Snawder et al. (1993) reported that PG inhibited metabolism of acetaminophen by CYP2E1 but not $1 \mathrm{~A} 2$ in mouse subcellular liver fractions in vitro, again suggesting a CYP2E1specific effect of the cosolvent. 
In vivo inhibition of metabolism by PG has previously been reported by Dean and Stock (1974), who reported that the co-solvent increased hexobarbital sleeping times and zoxazolamine paralysis in rats, after dosing with of $4 \mathrm{ml} / \mathrm{kg}$ twice daily for 3 days. These workers also reported inhibition of aminopyrine metabolism in hepatic microsomes from rats which had been pre-treated with 4ml/kg PG twice daily for three days (Dean and Stock, 1974), in contrast to the lack of effect seen in the APBT in the present study. This may be due to the fact that their study measured effects after repeated dosing, in contrast to the single acute dose of PG used in the present study. Indeed, Walters et al. (1993) reported inhibition of dramamine metabolism by PG in animals pre-treated with $50 \%$ PG, but no inhibitory effect was seen when naïve animals received PG for the first time. This highlights the possibility that effects on metabolism may be seen after multiple doses of a co-solvent or excipient, which do not occur after a single acute dose. This is important to bear in mind when interpreting the results of the present studies, as it is possible that doses of excipient which showed no effects after a single administration could affect metabolism after prolonged exposure of metabolising enzymes to the excipient during chronic dosing. 
Solutol HS15, at the commonly used dose of $90 \mathrm{mg} / \mathrm{kg}$, showed no significant effects on the APBT or EMBT, demonstrating that it did not affect metabolism by CYP3A or the variety of enzymes that metabolise aminopyrine at this dose. Solutol has been reported to inhibit clearance of the CYP3A substrate midazolam in rat liver microsomes and hepatocytes (Bravo Gonzalez et al., 2001; Bravo Gonzalez et al., 2004b). This was thought to be due to inhibition of CYP3A or binding of the drug to the excipient. However, Solutol had no significant effects on midazolam pharmacokinetics in the rat (Bittner et al., 2003a), attributed to the high hepatic extraction ratio of midazolam.

Solutol did significantly affect the in vivo pharmacokinetics of another CYP3A substrate, colchicine (Bittner et al., 2003b). At a dose of $100 \mathrm{mg} / \mathrm{kg}$, Solutol reduced CL by $55 \%$, increased $\mathrm{C}_{\max }$ by 2.4-fold, and increased colchicine excretion into urine by 35 -fold. The amount of demethylcolchicine in urine doubled, but this was not significant. There was also a non-significant $58 \%$ reduction in $\mathrm{V}$. No significant alteration in half-lives was seen. The authors concluded that the excipient had altered the metabolism and/or distribution of colchicine; inhibited metabolism was suggested as a likely cause, as Solutol also reduced the in vitro $\mathrm{CL}$ of colchicine in rat hepatocytes. However, our results suggest that inhibition of metabolism by CYP3A may not have been responsible. 
A possible explanation for this is that the effects of Solutol on colchicine pharmacokinetics may have been due to inhibition of PGp rather than CYP3A. Desrayaud et al. (1997) reported that the PGp inhibitor SDZ PSC 833 increased colchicine $A U C_{0-6 h}$, by 2.5fold and $C_{\max }$ by 2.2 -fold, and reduced $C L$ by $47 \%$. $V_{\beta}$ was reduced by $18 \%$, which was not significant. These effects are similar to those seen in Bittner et al's study, suggesting that P-Gp inhibition by Solutol may be at least partially responsible for the effects reported.

Bravo Gonzalez et al. (2004a) reported that Solutol inhibited active transport of colchicine into hepatocytes at concentrations above its critical micelle concentration (CMC), and suggested that the effects seen on in vivo colchicine pharmacokinetics in Bittner et al's study could be due to a combination of this and inhibition of metabolism. If this is the primary mechanism involved, it may be another explanation for the differences seen between their work and the present study. Another possible explanation for this difference is the manner of administration of the drugs and excipient; erythromycin and Solutol were administered separately in our experiment, whereas colchicine was formulated with the Solutol in their study. The authors do comment that an association between the drug and excipient, thus 
limiting availability of the drug for clearance and distribution processes, was a plausible mechanism for the changes seen.

Some workers have successfully used 1 -methyl- ${ }^{14} \mathrm{C}$-caffeine in breath tests. Recent works published in rat have used this labelling position, but did not determine $t_{1 / 2}$, using cumulative $\%{ }^{14} \mathrm{CO}_{2}$ exhaled over two hours as their outcome measure (Arnaud, 1976; Bastien and Villeneuve, 1998; Provencher et al., 1999; Leblond et al., 2000). Arnaud et al. (1976) reported that $6.6 \%$ of the total caffeine dose administered to rats was excreted in urine as theobromine, the product of caffeine 1-demethylation. Paraxanthine, the product of 3-demethylation, excreted in urine accounted for $9.2 \%$ of the dose (Arnaud, 1976). As the product of 3-demethylation appears to be produced in larger amounts, it may be that 3 -methyl- ${ }^{14} \mathrm{C}$-caffeine would be more suitable for use in breath tests. Indeed, Schaad et al. (1995) used 3-methyl- ${ }^{14} \mathrm{C}-$ caffeine in their breath test, and produced CER vs. time curves which demonstrated a decline in CER over four hours, unlike those we produced with 1 -methyl- ${ }^{14} \mathrm{C}$-caffeine. It would appear therefore that 3-methyl- ${ }^{14} \mathrm{C}$-caffeine, rather than 1 -methyl- ${ }^{14} \mathrm{C}$-caffeine, is more suitable for use in breath tests in rats.

It is important to bear in mind that the breath test will also be sensitive to changes in metabolism which are not caused by enzyme 
inhibition, such as changes in protein binding, or inhibition of transport proteins. Kurnik et al. (2006) reported that the EMBT can also be affected by P-Gp inhibition as well as alterations in CYP3A activity in humans. Administration of tariquidar, an inhibitor of P-Gp but not CYP3A, significantly increased the $\%$ dose exhaled as ${ }^{14} \mathrm{CO}_{2}$ over 1 hour, giving a median increase of 2.3 -fold. $T_{\max }$ and $C_{\max }$ were also increased. Clearance of midazolam (a substrate for CYP3A but not P-Gp) was not affected, confirming that CYP3A induction was not responsible for the effects of tariquidar. Thus, P-Gp inhibition gave the appearance of CYP3A induction in the EMBT. This was attributed to inhibited biliary elimination of erythromycin increasing exposure to metabolic enzymes in the hepatocyte. The authors concluded that P-Gp can affect the outcome of the EMBT, and that as such the test was not suitable to compare CYP3A phenotypes between individuals, but could be used to examine the effects of inhibitors and inducers within a subject, provided the potential confounding influence of $\mathrm{P}-\mathrm{Gp}$ inhibition was taken into account. This effect does not appear to have occurred in the present study, as no apparent enzyme induction was seen. However it does demonstrate the need to remember that the breath test can also be affected by other in vivo factors, besides inhibition of metabolising enzymes. 
Mico et al. (1985) reported that NDMA has a high extraction ratio (92\%) when administered in low doses, meaning that changes in its metabolism are likely to be due to changes in hepatic blood flow rather than enzyme activity. If the compound does have a high extraction ratio, the NDMA BT will be less sensitive to changes in enzyme activity; large reductions in intrinsic clearance would be needed to produce only small reductions in $t_{1 / 2}$. One group topped up the ${ }^{14} \mathrm{C}$-NDMA dose in a breath test with cold NDMA in an attempt to overcome this, but did not repeat this practice in a later study (Bastien and Villeneuve, 1998; Bastien et al., 2000). However, Mico et al. (1985) state that their CL of $39 \mathrm{ml} / \mathrm{min} / \mathrm{kg}$ approaches liver blood flow in the rat, whereas in fact it is only around $50 \%$ of the reported value for this parameter (69$77 \mathrm{ml} / \mathrm{min} / \mathrm{kg}$ ) (Walton et al., 2001). It therefore appears that the blood flow used in Mico et al's calculations for extraction ratio may have been erroneous; however, the calculations are not reported to enable them to be checked. Using the equation:

$$
C L_{H}=\left(Q_{H} \cdot f_{u} \cdot C L_{i n t}\right) /\left(Q_{H}+f_{u} \cdot C L_{i n t}\right)
$$

with the $\mathrm{CL}$ determined in this paper and reported values for rat liver blood flow, it can be calculated that a $50 \%$ reduction in $\mathrm{Cl}_{\text {int }}$ for this compound would give rise to a reduction in $\mathrm{CL}_{H}$ of approximately $32 \%$. It therefore appears that the clearance of NDMA would respond to large changes in $\mathrm{CL}_{\text {int, }}$ and thus enable the 
detection of changes in liver metabolic capacity. However this again highlights the need to consider factors other than changes in metabolism which could affect the results of the breath tests.

\subsubsection{Conclusions}

These results demonstrate that excipients can affect the in vivo metabolism of some drugs, after a single acute administration of doses commonly used in pre-clinical studies. Their potential to do this should be borne in mind when selecting excipients for use in intravenous drug formulations, as inappropriate selection could lead to a false impression of drug half-life, and affect metabolism of concomitantly administered drugs. However, it appears that, for DMSO and Solutol, single acute administrations of the doses used in pre-clinical studies do not have an appreciable effect on metabolic capacity. The results also demonstrate that excipients which can inhibit metabolism in vitro do not necessarily do this in vivo, at commonly used doses. 


\subsubsection{Further work}

It would be of interest to perform breath tests on a wider selection of excipients. The effects of other doses of DMSO on the breath tests could also be investigated, to determine at what dose significant inhibitory effects start to occur, and whether they occur at different doses of DMSO for different enzymes. It would also be of interest to investigate the effects of repeated doses of excipients on in vivo metabolism. However, time and resource limitations prevent us from doing this.

The caffeine breath test could also be repeated with 3 -methyl- ${ }^{14} \mathrm{C}$ caffeine, to see if this is more successful. However, we are limited in the amount of time and resources that can be devoted to this set of experiments, as many other aspects of pharmacokinetics remain to be investigated.

Investigation into the effects of Solutol on other aspects of drug pharmacokinetics, to clarify the mechanism of the effects seen in Bittner et al's study, would also be of interest. Investigation into the effects of excipients on plasma protein binding would also be useful, as increased protein binding could also be a potential source of reduced metabolism for some drugs. 
Chapter 3:

Effects of excipients on $\alpha_{1}$-acid glycoprotein plasma levels in vivo 


\section{Contents:}

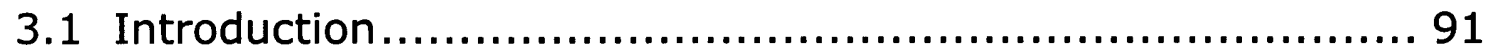

3.2 Methods and materials ......................................... 96

3.2.1 Materials................................................. 96

3.2.2 Methods..................................................... 97

3.3 Results........................................................ 101

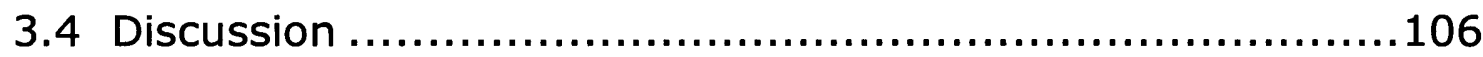

3.4.1 Conclusions ................................................. 115

3.4.2 Further work ............................................ 115 


\subsection{Introduction}

$\alpha_{1}$-acid glycoprotein ( $\alpha_{1}$-AGP) is one of the major drug binding proteins in plasma, binding mainly basic and neutral drugs (Kremer et al., 1988; Israili and Dayton, 2001). Plasma levels of this protein are low compared to albumin - normal plasma levels in humans are $50-140 \mathrm{mg} / 100 \mathrm{ml}$, compared to $4 \mathrm{~g} / 100 \mathrm{ml}$ of albumin (Kremer et al., 1988; Israili and Dayton, 2001). Despite this, $\alpha_{1}$-AGP is the major binding protein for some drugs, due to a high affinity for the compounds that it binds (Kremer et al., 1988; Israili and Dayton, 2001). As $\alpha_{1}$-AGP is an acute phase protein, plasma levels increase significantly during an acute phase response. This has been demonstrated in various disease states, infection and inflammation. For example, plasma $\alpha_{1}$-AGP levels were increased by $250 \%$ in Crohn's disease (Piafsky et al., 1978), 226\% in rheumatoid arthritis (Piafsky et al., 1978), 248\% in bacterial infection (Voulgari et al., 1982), and $188 \%$ in malaria (Voulgari et al., 1982). Levels have also been reported to increase after treatment with certain drugs, such as amitryptyline (Baumann et al., 1982).

Such changes in plasma $\alpha_{1}$-AGP concentrations can affect the protein binding of drugs which bind to this protein. For example, there was a significant negative correlation between the percentage 
of unbound propranolol and plasma $\alpha_{1}$-AGP concentration in a group of patients and healthy volunteers (Piafsky et al., 1978). Patients suffering from Crohns disease, arthritis, and renal failure with complications, had elevated plasma $\alpha_{1}$-AGP levels and reduced free fraction of propranolol compared to healthy controls (Piafsky et al., 1978). Inducing inflammation in rats with turpentine oil also elicits significant rises in plasma $\alpha_{1}$-AGP levels, and this model has been used to demonstrate the effects of increased $\alpha_{1}$-AGP levels on drug pharmacokinetics. Sugihara et al. (1993) used this method to investigate the effects of increased $\alpha_{1}$-AGP levels on quinidine pharmacokinetics in rats. Levels of $\alpha_{1}$-AGP were increased by 3 -fold 16 hours after turpentine treatment, and 15 -fold 48 hours after turpentine treatment. There was no change in albumin levels. Quinidine plasma free fraction was reduced by $30 \% 16$ hours after turpentine treatment, and $76 \% 48$ hours after turpentine treatment. The ratio of bound to free quinidine correlated with plasma $\alpha_{1}$-AGP concentration. Blood clearance of quinidine was reduced (65\% reduction 48 hours after turpentine treatment, at a quinidine dose of $12.5 \mathrm{mg} / \mathrm{kg}$ ). Hepatic extraction ratio was also reduced, and this was suggested as the mechanism by which the increased $\alpha_{1}$-AGP levels reduced quinidine blood clearance. Distribution of quinidine was also altered, with significant reductions in $V_{d, s s}(79 \%$ reduction 48 hours after turpentine treatment, at a quinidine dose of $12.5 \mathrm{mg} / \mathrm{kg}$ ), and corresponding reductions in tissue:plasma 
partition coefficients for the lung, kidney, liver, heart and spleen. Significant correlations were also obtained between the tissue:plasma partition coefficients and plasma free fraction of quinidine. These results demonstrate that increased binding to $\alpha_{1}$ AGP reduced the distribution of quinidine to the tissues.

Matsushima et al. (2000) investigated the effects of increased $\alpha_{1}$ AGP levels on the pharmacokinetics of tamsulosin in rats. Pretreatment with turpentine oil caused an 8-fold increase in plasma $\alpha_{1}$-AGP levels ( 2 days after turpentine treatment), accompanied by a decrease in tamsulosin fraction unbound from 0.273 to 0.1 . Albumin levels were reduced by around $15 \%$, which was significant in one of two groups of rats. Plasma levels of tamsulosin were increased in the turpentine treated rats, and $V_{d, s s}$ reduced by $59 \%$. Plasma clearance was reduced by $47 \%$. As clearance of tamsulosin is hepatic blood flow limited (Matsushima et al. 2000), blood clearance is expected to be insensitive to changes in protein binding. The reduction in plasma clearance was therefore attributed to the reduction in blood:plasma concentration ratio caused by increased plasma $\alpha_{1}-\mathrm{AGP}$, rather that any alteration in hepatic extraction ratio.

Murai-Kuyisha et al. (1993) demonstrated that turpentine oil treatment increased $\alpha_{1}$-AGP concentrations in rats by 13 -fold ( 2 
days after treatment). Albumin concentrations were reduced by $30 \%$, but total plasma protein concentration remained the same. Plasma concentrations of propranolol and oxprenolol were increased by turpentine oil pre-treatment. As seen in the studies described above, plasma clearance and volume of distribution were reduced for both enantiomers of these drugs, with the enantiomer that bound most strongly to $\alpha_{1}$-AGP most affected. Blood clearances were not reported; however, as both drugs have moderate clearance values (Murai-Kuyisha et al., 1993), blood clearance can be affected by changes in $f_{u}$. The reduction in plasma clearance reported may therefore represent a change in the hepatic extraction ratio of the drugs, rather than simply the alteration in plasma protein binding caused by increased $\alpha_{1}$-AGP concentrations. No effect on acebutolol pharmacokinetics was seen, as this drug does not bind to $\alpha_{1}$-AGP. These examples demonstrate that changes in $\alpha_{1}$-AGP levels can significantly affect drug plasma protein binding and pharmacokinetics.

It is possible that excipients could induce an acute-phase response, thereby increasing plasma $\alpha_{1}$-AGP concentrations and potentially affecting drug plasma protein binding and pharmacokinetics. Ivanovic Matic et al. (2004) demonstrated increased plasma $\alpha_{1}$-AGP levels caused by DMSO. A dose of $0.9 \mathrm{ml} / \mathrm{kg}$ DMSO administered to rats caused an increase in the plasma concentrations of acute phase 
proteins, including a $630 \%$ increase in $\alpha_{1}$-AGP concentrations, 24 hours after administration of the excipient. Although this dose of DMSO is somewhat higher than the doses typically used in preclinical studies, it raises the possibility that this response could occur with formulation levels of excipient. The profound changes in pharmacokinetics caused by increased plasma $\alpha_{1}$-AGP described above demonstrate that this is an area worth investigating.

An accurate method of quantifying $\alpha_{1}$-AGP in plasma is necessary if we are to investigate the effects of excipients on levels of this protein. A variety of methods are described in the literature. Immunological methods, using antibodies raised against rat $\alpha_{1}$-AGP, are commonly used, as they are by nature specific for the protein. Various immunological methods have been used in the literature, including radial immunodiffusion (Arnold and Meyerson, 1990; Sugihara et al., 1993; Svelander et al., 2004; Projean et al., 2005), ELISA (Murai-Kuyisha et al., 1993; Matsushima et al., 2000), and immunoelectrophoresis (Ivanovic Matic et al., 2004). Commercial radial immunodiffusion and ELISA assays for detection of rat $\alpha_{1}$-AGP are available. Immamura et al. (1994) described a fluorimetric method using quinaldine red for detection of human $\alpha_{1}$-AGP. This method could be adapted for rat $\alpha_{1}$-AGP, but the cost of purified rat plasma proteins for making reference serum would make this very expensive. A commercial radial immunodiffusion assay kit will be 
used to measure $\alpha_{1}$-AGP levels in the present study. This test is easy to perform (single-step process) and to interpret, and will allow accurate quantification of the amount of $\alpha_{1}$-AGP in the plasma samples.

This series of experiments aims to investigate the effects of excipients on plasma levels of $\alpha_{1}$-AGP in rats.

\subsection{Methods and materials}

\subsubsection{Materials}

DMSO, PG, HP- $\beta-C D$, Tween 80 , and EDTA were obtained from Fisher, UK. Solutol HS15 was a kind gift from AstraZeneca, Alderley Park, UK. Radial immunodiffusion plates for detection of $\alpha_{1}$-AGP and the accompanying standard solutions were obtained from Biognosis Ltd, Hailsham, UK. BCA protein assay reagents were obtained from Pierce, Illinois, USA. 


\section{Animals}

Male Wistar rats (200-285g) (Harlan) were group housed on a 12 hour light/dark cycle, with access to water and standard laboratory rodent diet ad libitum.

\subsubsection{Methods}

\section{Dosing}

Groups of three rats per treatment were used. Single and multiple dosing regimens investigated are shown in Table 3.1. For multiple dosing regimens, two doses were given $24 \mathrm{~h}$ apart. All doses were administered under isoflurane anaesthesia.

Table 3.1: Single and multiple dosing regimens investigated.

\begin{tabular}{|l|l|c|l|c|}
\hline $\begin{array}{c}\text { Dosing } \\
\text { regimen }\end{array}$ & Excipient & $\begin{array}{c}\text { Dose } \\
\text { (mg/kg) }\end{array}$ & Administered as & Route \\
\hline Single & DMSO & 440 & $20 \%$ solution, $2 \mathrm{ml} / \mathrm{kg}$ & i.p. \\
dose & DMSO & 990 & $45 \%$ solution, $2 \mathrm{ml} / \mathrm{kg}$ & i.p. \\
& PG & 829 & $40 \%$ solution, $2 \mathrm{ml} / \mathrm{kg}$ & i.p. \\
& Saline ctrl & - & $2 \mathrm{ml} / \mathrm{kg}$ & i.p. \\
\hline Multiple & Solutol & 100 & $12.5 \%$ solution, $0.8 \mathrm{ml} / \mathrm{kg}$ & i.v. \\
doses & Tween 80 & 85 & $10 \%$ solution, $0.8 \mathrm{ml} / \mathrm{kg}$ & i.v. \\
& HP- $\beta-C D$ & 360 & $45 \%$ solution, $0.8 \mathrm{ml} / \mathrm{kg}$ & i.v. \\
& DMSO & 2200 & $100 \%$ solution, $2 \mathrm{ml} / \mathrm{kg}$ & i.p. \\
\hline
\end{tabular}




\section{Blood collection}

A baseline blood sample was collected from each rat, to ensure that all rats had normal $\alpha_{1}$-AGP levels before dosing commenced (this was not possible in three of the rats due to sampling difficulties; however, these rats did not exhibit elevated $\alpha_{1}$-AGP levels after excipient treatment, so the results were not affected by this). Blood samples were then collected $48 \mathrm{~h}$ after the first dose of excipient. All blood samples were taken under isoflurane anaesthesia. EDTA (5\%) was used as an anticoagulant. Blood was centrifuged at $12,000 \mathrm{~g}$ for until required for analysis. The penile vein was used for dosing and sampling.

\section{Determination of $\alpha_{1}$-AGP levels}

Initially, pooled plasma from each group of rats was analysed for $\alpha_{1}$-AGP levels in order to minimise use of the assay kit, and $\alpha_{1}$-AGP levels were then determined in plasma from individual rats if this proved necessary for clarification of results. For the multiple dosing experiments, and for the PG repeat, post-dosing samples from individual rats were analysed rather than pooled blood. Concentrations of $\alpha_{1}$-AGP in plasma were determined using a commercial radial immunodiffusion assay, according to the 
manufacturer's instructions. $5 \mu$ l aliquots of plasma and the two standard solutions provided with the kit ( 250 and $1000 \mu \mathrm{g} / \mathrm{ml}$ of $\alpha_{1}$ AGP) were pipetted into wells on the immunodiffusion plates. The plate was incubated at $37^{\circ} \mathrm{C}$ for 24 hours in a humidified box. The diameter of the precipitin ring resulting from each sample was measured, and a calibration line constructed using the diameters of the rings from the two standard solutions. The diameters of the rings resulting from the plasma samples were compared to this calibration line to determine their $\alpha_{1}$-AGP concentration. Samples were initially assayed in duplicate; however, it became apparent that the kit was suitably reproducible for this not to be necessary.

\section{Measurement of total protein levels}

Total protein levels in plasma samples were determined using the BCA protein assay. Plasma samples were diluted $1 / 50$ in doubledistilled water. The working reagent was prepared according to the manufacturer's instructions immediately before use. Protein standards of known concentrations were prepared from a stock solution of bovine serum albumin supplied with the kit, according to the manufacturer's instructions. $25 \mu$ aliquots of plasma and protein standards, plus four water blanks, were pipetted into individual wells in a 96-well plate. Duplicate measurements were performed 
for each sample. $200 \mu$ l of the working reagent was added to each well, and the plate was incubated at $37^{\circ} \mathrm{c}$ for 30 minutes, subject to shaking on an orbital shaker. After incubation, the plate was removed and allowed to cool before measuring the absorbance of each well at 570nm on an Anthos HT2 microplate reader.

The average blank absorbance was subtracted from the average absorbance for each sample. The corrected absorbances for the plasma samples were compared to a calibration line constructed from the protein standards to determine the protein concentration in each sample. A one-way ANOVA was used to assess differences in total protein concentration between groups. 


\subsection{Results}

Previous studies in rats have reported normal $\alpha_{1}$-AGP levels in the range of $59-142 \mu \mathrm{g} / \mathrm{ml}$ (Murai-Kuyisha et al., 1993; Sugihara et al., 1993; Matsushima et al., 2000). All of the baseline $\alpha_{1}$-AGP levels in the rats in the present study were below the upper limit of this range. The lower limit of measurement for the batch of the $\alpha_{1}$-AGP immunodiffusion assay kit received was $100 \mu \mathrm{g} / \mathrm{ml}$ (the advertised limit on which selection of the kit was based was $50 \mu \mathrm{g} / \mathrm{ml}$, however this was increased for the batch received). This meant that normal levels $\alpha_{1}$-AGP in plasma tended to be either at or below the limit of accurate measurement. However, it was still possible to say with certainty that the levels were not abnormally high. Elevated plasma $\alpha_{1}$-AGP levels were within the detection limits, and so could be accurately quantified.

\subsubsection{Single dose studies}

The effects of single ip doses of $440 \mathrm{mg} / \mathrm{kg}$ and $990 \mathrm{mg} / \mathrm{kg}$ DMSO, $829 \mathrm{mg} / \mathrm{kg}$ PG, and a saline control, on $\alpha_{1}$-AGP levels in rats are shown in Figure 3.1. No standard deviations are indicated, as the levels were measured in pooled plasma. 


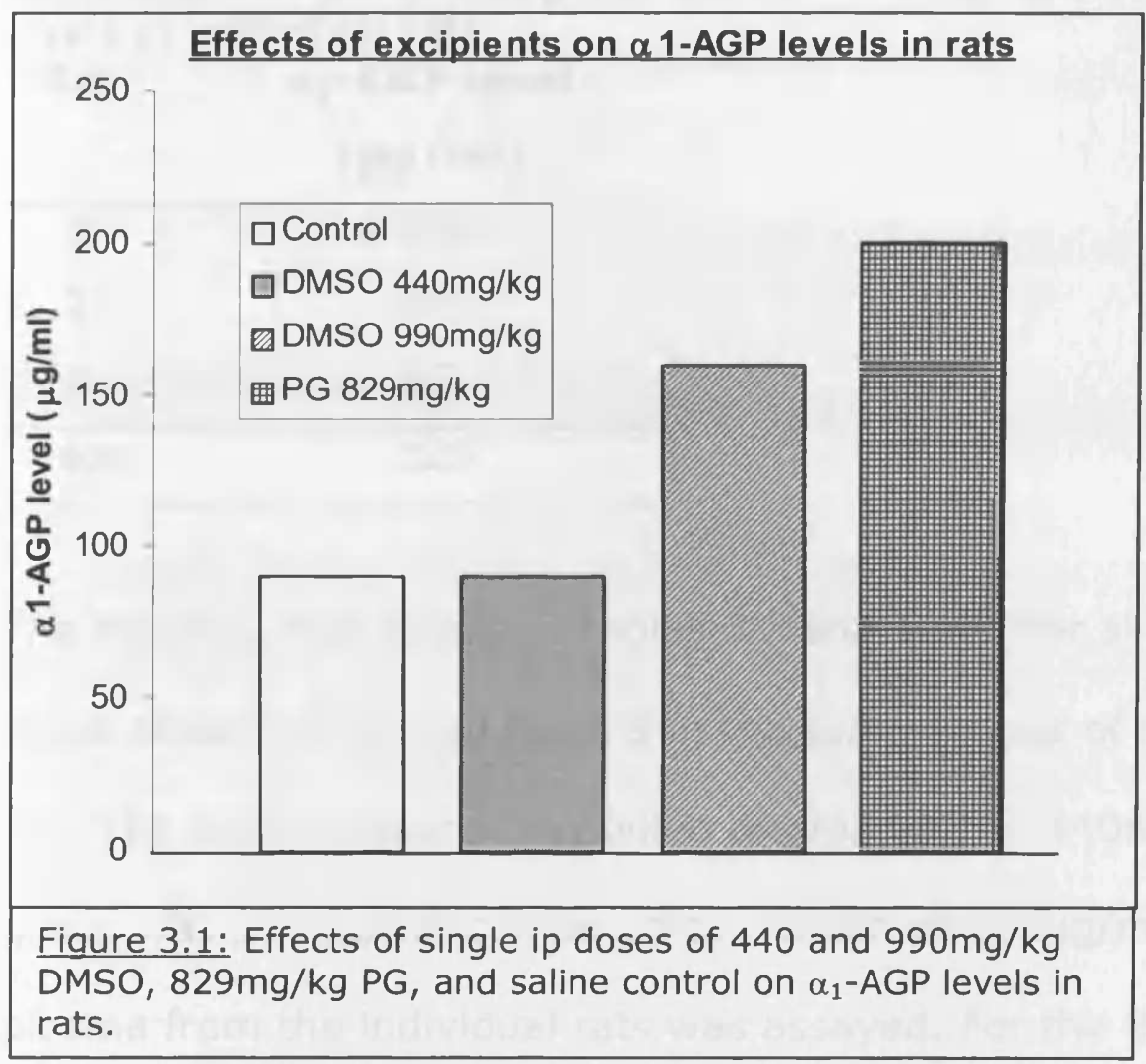

Levels of $\alpha_{1}$-AGP in individual rats in the $440 \mathrm{mg} / \mathrm{kg}$ DMSO and $829 \mathrm{mg} / \mathrm{kg}$ PG groups were then measured to investigate the increases seen in these groups (Tables 3.2 and 3.3).

Table 3.2: Effects of a single ip dose of $440 \mathrm{mg} / \mathrm{kg}$ DMSO on $\alpha_{1}$-AGP levels in individual rats.

\begin{tabular}{cc}
\hline Rat & $\begin{array}{c}\boldsymbol{\alpha}_{\mathbf{1}} \text {-AGP level } \\
(\boldsymbol{\mu} \mathbf{g} / \mathbf{m l})\end{array}$ \\
\hline 1 & 320 \\
2 & 115 \\
3 & 115 \\
\hline Mean & 183 \\
\hline
\end{tabular}


Table 3.3: Effects of a single ip dose of $829 \mathrm{mg} / \mathrm{kg}$ PG on $\alpha_{1}$-AGP levels in individual rats.

\begin{tabular}{cc} 
Rat & $\begin{array}{c}\boldsymbol{\alpha}_{\mathbf{1}} \text {-AGP level } \\
(\boldsymbol{\mu g} / \mathbf{m l})\end{array}$ \\
\hline 1 & 105 \\
2 & 475 \\
3 & 95 \\
\hline Mean & 225 \\
\hline
\end{tabular}

The mean $\alpha_{1}$-AGP levels in Tables 3.2 and 3.3 differ slightly from those shown for pooled plasma in the same groups of rats in Figure 3.1. The level measured in pooled plasma for the $440 \mathrm{mg} / \mathrm{kg}$ DMSO group was $160 \mu \mathrm{g} / \mathrm{ml}$, compared to a mean of $183 \mu \mathrm{g} / \mathrm{ml}$ when plasma from the individual rats was assayed. For the $829 \mathrm{mg} / \mathrm{kg}$ PG group, the $\alpha_{1}$-AGP level measured in pooled plasma was $200 \mu \mathrm{g} / \mathrm{ml}$, compared to a mean of $225 \mu \mathrm{g} / \mathrm{ml}$ for the individual rats. These differences are likely to be due to intrinsic variability in the assay used to determine $\alpha_{1}$-AGP levels. However, they are not large enough to suggest that the results of the study could have been significantly influenced by such variability.

\section{PG repeat}

A further six rats were treated with a single dose of $829 \mathrm{mg} / \mathrm{kg}$ PG to further investigate the effect seen above. Plasma levels of $\alpha_{1}$-AGP 
were not increased in any of the rats in this group after treatment with PG. The mean plasma $\alpha_{1}$-AGP level was $71.7 \mu \mathrm{g} / \mathrm{ml}$.

\subsubsection{Multiple dose studies}

The effects of multiple doses ( 2 doses 24 hours apart) $100 \mathrm{mg} / \mathrm{kg}$

Solutol HS15 iv, $85 \mathrm{mg} / \mathrm{kg}$ Tween $80 \mathrm{iv}, 360 \mathrm{mg} / \mathrm{kg}$ HP- $\beta-C D$ iv, and $2200 \mathrm{mg} / \mathrm{kg}$ DMSO (neat) ip, on plasma levels of $\alpha_{1}$-AGP are shown in Figure 3.2.

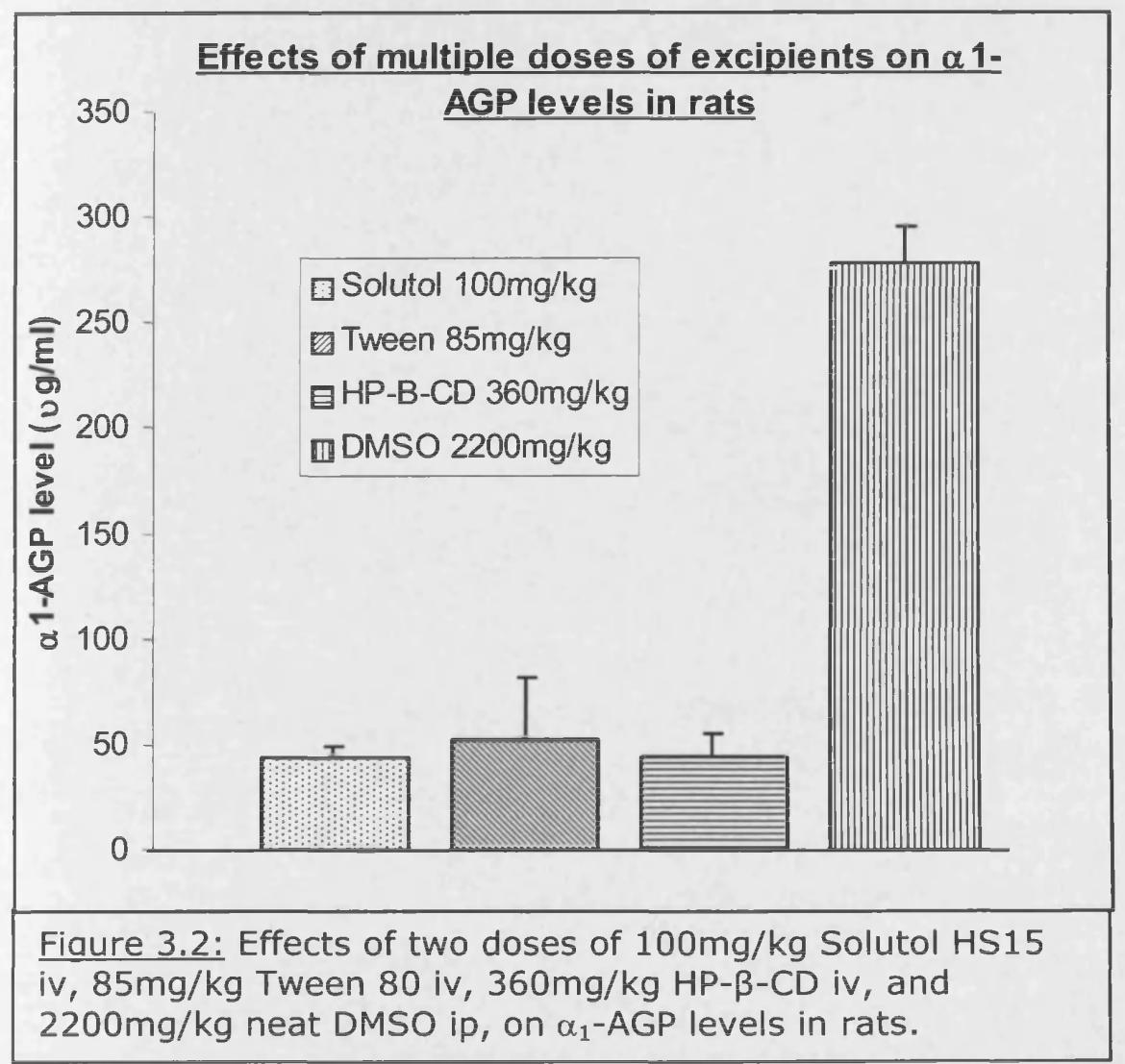




\section{$\underline{\text { 3.3.3 Total protein levels }}$}

The effects of excipient dosing on total protein levels in rat plasma are shown in Table 3.4. There were no significant differences between any of the groups.

Table 3.4: Effects of excipients on levels of total plasma protein in rats.

\begin{tabular}{|ll|c|}
\hline \multicolumn{2}{|c|}{ Dosing regimen } & $\begin{array}{c}\text { Total protein concn } \\
(\mathrm{mg} / \mathrm{ml})(\mathrm{mean} \pm \mathrm{SD})\end{array}$ \\
\hline Single & DMSO $440 \mathrm{mg} / \mathrm{kg}$ & $58.32( \pm 4.59)$ \\
doses & DMSO $990 \mathrm{mg} / \mathrm{kg}$ & $50.34( \pm 3.69)$ \\
& Saline ctrl & $58.58( \pm 8.05)$ \\
& PG $829 \mathrm{mg} / \mathrm{kg}$ & $58.23( \pm 2.75)$ \\
\hline Repeat & Solutol $100 \mathrm{mg} / \mathrm{kg}$ & $57.75( \pm 10.00)$ \\
doses & Tween $8085 \mathrm{mg} / \mathrm{kg}$ & $59.49( \pm 3.36)$ \\
& HP- $\beta-C D 360 \mathrm{mg} / \mathrm{kg}$ & $63.22( \pm 6.59)$ \\
& DMSO $2200 \mathrm{mg} / \mathrm{kg}$ & $65.48( \pm 4.30)$ \\
\hline PG repeat & PG $829 \mathrm{mg} / \mathrm{kg}$ & $56.44( \pm 5.36)$ \\
\hline
\end{tabular}




\subsection{Discussion}

In the single dose experiments, increases in $\alpha_{1}$-AGP levels were seen in the groups receiving $990 \mathrm{mg} / \mathrm{kg}$ DMSO and $829 \mathrm{mg} / \mathrm{kg}$ PG. However, the magnitude of these increases was much smaller than expected (1.8-fold and 2.2-fold for DMSO and PG, respectively). This suggested that they may be due to a large rise in $\alpha_{1}$-AGP level in an individual rat in each group, rather than an increase in levels across the whole group. When plasma from individual rats in these groups was assayed, it became apparent that the increases in $\alpha_{1}$ AGP levels in the pooled plasma from both PG and DMSO groups were due to a large rise in a single rat in each group, as suspected. The lack of effect seen in the saline control group suggests that these increased levels were not due to the experimental procedures used. There were no overt signs of illness or injury in either of the rats where the increase occurred, and they were housed in different cages. The PG rats weight did not increase as fast as the others, but the weight of the DMSO rat increased as expected. It is possible that the increases $\alpha_{1}$-AGP levels were due to an idiosyncratic effect of the excipients, to which some rats are more susceptible than others. If this is the case, it would introduce an extra element of variability into pharmacokinetic studies where these excipients were used at these doses, as the effect would not occur in a predictable manner in all rats. There was no increase in $\alpha_{1}$-AGP levels in the 
group receiving $440 \mathrm{mg} / \mathrm{kg}$ DMSO, suggesting that this dose of the excipient does not induce an acute phase response.

Treatment with $1 \mathrm{mg} / \mathrm{g}(0.9 \mathrm{ml} / \mathrm{kg})$ DMSO has been reported to increase plasma $\alpha_{1}$-AGP levels by $630 \%$ in male Wistar rats (Ivanovic Matic et al., 2004). As the higher dose of DMSO used in the single dose studies was virtually equivalent to this, it is of interest that an increase in $\alpha_{1}$-AGP levels was only observed in one of the three rats in the present study. In Ivanovic Matic et al's study, $\alpha_{1}$-AGP levels were measured 24 hours after excipient treatment rather than 48 hours after. The acute phase response begins to subside after $24-48 \mathrm{~h}$ without prolonged stimulation (Baumann et al., 1982; Gruys et al., 2005). Although the half-life of rat $\alpha_{1}$-AGP is not reported, the half-life of human $\alpha_{1}$-AGP in rats is 17 hours (Herve et al., 2003). This suggests that if an increase of the magnitude described by Ivanovic Matic et al. occurred in the present study, it should have been detectable at $48 \mathrm{~h}$, even if $\alpha_{1}$ AGP levels had begun to subside. Indeed, the work of Sugihara et al. demonstrates that $\alpha_{1}$-AGP levels were more elevated at $48 \mathrm{~h}$ than at $16 \mathrm{~h}$ after turpentine treatment, so it seems reasonable to expect that an effect would have been measurable at $48 \mathrm{~h}$ in the present study had one occurred. 
It may be that the dilution of the DMSO dose with saline in the present study attenuated its effects (this was done for ease of injection, and so that the same volume was injected for all ip treatments). Dilution may have reduced the local inflammatory effects of DMSO, impairing its ability to induce the acute phase response. It is also possible that the rate of DMSO absorption from the peritoneal cavity was retarded by dilution with the saline by altering the concentration gradient for diffusion, but as DMSO crosses membranes easily this seems unlikely to be the reason for the lack of effect seen. It is also possible that the rats used in their study were for some reason more susceptible to the effects of DMSO, due to genetic differences (the source of the rats used for their study was not stated). The weight range of the rats used in Ivanovic Matic et al.'s study (200-300g) was consistent with the rats used here.

The same route of administration was used in the present study and Ivanovic Matic et al.'s work, eliminating this as a potential reason for the difference in results. However, it is important to remember that the ability of excipients to induce the acute phase response may differ dependant upon route of administration. The i.v. and i.p. routes used in the current study were selected to represent current common practice in the administration of dosing solutions. However, the subcutaneous or intramuscular routes are often used 
for turpentine oil when aiming to initiate an acute phase response. If the excipients were administered by these routes, they may cause a greater degree of local inflammation, and so initiate an acute phase response and a corresponding increase in $\alpha_{1}$-AGP levels at doses where no effect is seen by the i.v. and i.p. routes.

It was decided to repeat the single dose PG experiment with a larger group of animals. However, none of the six animals in this group showed any change in $\alpha_{1}$-AGP levels after dosing. The increase in $\alpha_{1}$-AGP levels seen in a single rat in the first group therefore may not be due to treatment with PG, unless the incidence of this effect is very low.

PG has been reported to cause nephrotoxicity in some patients who received the cosolvent as an injection vehicle (Hayman et al., 2003; Yaucher et al., 2003). Yaucher et al. (2003) reviewed 128 patients from their hospitals intensive care and burns units who had received intravenous infusions of lorazepam, which contains PG in the injection vehicle. Eight of the patients developed elevated serum creatinine $(\mathrm{Cr})$, attributed to $\mathrm{PG}$, during lorazepam treatment. This occurred after 3-60 days treatment with lorazepam/PG (median 9 days), and was associated with PG levels of $186-3450 \mu \mathrm{g} / \mathrm{ml}$ (mean $1103 \mu \mathrm{g} / \mathrm{ml}$ ). The dose of PG used in the present study would give theoretical maximum blood PG levels of $1.30 \mathrm{mg} / \mathrm{ml}$, assuming 
distribution into total body water. Although this is much higher than the levels where toxicity occurred in Yaucher et al.'s study, PG levels in their patients would be sustained, as the excipient was administered by infusion rather than as a bolus. The fact that renal toxicity of PG only occurred in eight out of 128 patients demonstrates that this is not a universal effect. Also, the patients susceptibility to the toxic effects of PG may have been enhanced by the fact that they were seriously ill. So although renal toxicity of PG has been reported, it does not occur in all patients, and the PG levels and duration of dosing at which this occurs are variable. This may be consistent with the fact that an increase in $\alpha_{1}$-AGP level was observed in only one of the nine rats dosed with this excipient in the present study.

After multiple dosing (2200mg/kg ip for 2 days), DMSO increased $\alpha_{1}$-AGP levels in all rats, to an average of $278 \mu \mathrm{g} / \mathrm{ml}$. This demonstrates that the excipient can induce the acute phase response in rats, in accordance with the conclusions of Ivanovic Matic et al. (2004). Such a large increase in $\alpha_{1}$-AGP concentrations is likely to affect the protein binding of drugs which bind to this protein, and hence potentially alter their pharmacokinetics. This also acts as a positive control, demonstrating that our methods can detect increases in $\alpha_{1}$-AGP concentrations. 
Solutol HS15, at a dose of $100 \mathrm{mg} / \mathrm{kg}$ iv for 2 days, did not affect $\alpha_{1}$ AGP levels in rat plasma. It appears that this is not a mechanism by which the excipient is likely to affect drug pharmacokinetics in preclinical studies. Bittner et al. (2003b) reported that Solutol caused a 35 -fold increase in renal excretion of colchicine in rats, and suggested renal toxicity of the excipient as a possible mechanism for this. The dose of Solutol used in their study $(5 \% 2 \mathrm{ml} / \mathrm{kg}$, i.e. $100 \mathrm{mg} / \mathrm{kg}$ ) was equivalent to ours. The results of the present study suggest that, if renal toxicity was the mechanism for this effect, it was not severe enough to induce the acute phase response, and hence increase plasma $\alpha_{1}$-AGP levels.

Tween 80 , at a dose of $85 \mathrm{mg} / \mathrm{kg}$ for 2 days, did not affect plasma levels of $\alpha_{1}$-AGP. This excipient is commonly used in pre-clinical studies at doses up to $5 \% 5 \mathrm{ml} / \mathrm{kg}$, equivalent to $266 \mathrm{mg} / \mathrm{kg}$ (Marcel DeMatas, personal communication). However, this is a maximum dose of Tween, and lower doses are likely to be used if they enable formulation of a suitable dosing solution. The dose of Tween used in the present study was limited by the volume that could be injected via the penile vein; it was not possible to further increase the concentration of the solution as this increased its viscosity, and made reliable injection difficult. Although it was not possible to investigate the maximum dose used in pre-clincal studies in this work, it was still worthwhile to investigate the effects of this 
excipient on $\alpha_{1}$-AGP levels, as this question is not addressed in the literature. Tween 80 is rapidly metabolised by plasma esterases; van Tellingen et al. (1999) reported that plasma levels in mice fell from $0.7 \% \mathrm{v} / \mathrm{v}$ to less than $0.05 \%, 15$ minutes after dosing. This rapid decline in plasma levels does not preclude the possibility of the excipient affecting $\alpha_{1}$-AGP levels; it is possible that one of its metabolites could have an effect. However, this does not appear to be the case at the dose used here.

No effect on plasma $\alpha_{1}$-AGP levels was seen with HP- $\beta-C D$ (360mg/kg for 2 days). In pre-clinical studies, this excipient is commonly used at doses up to $45 \% 4 \mathrm{ml} / \mathrm{kg}$, equivalent to $1800 \mathrm{mg} / \mathrm{kg}$ (Marcel DeMatas, personal communication). The volume of solution that could be injected into the penile vein again limited the dose of excipient used in the current study. However, $4 \mathrm{ml} / \mathrm{kg}$ is a maximum dose volume, and lower doses are likely to be used in studies if they provide sufficient increases in drug solubility. The results obtained in the present study demonstrate that this excipient does not affect $\alpha_{1}$-AGP levels at doses of $360 \mathrm{mg} / \mathrm{kg}$ for 2 days. Gould and Scott (2005) reported minor histopathological changes in the liver, kidney, lungs and spleen after 4 days dosing with an $11.25 \%$ solution of $\mathrm{HP}-\beta-\mathrm{CD}$ at $2 \mathrm{ml} / \mathrm{kg} / \mathrm{day}$. This is equivalent to $225 \mathrm{mg} / \mathrm{kg} /$ day. The fact that no change in $\alpha_{1}$-AGP levels was seen with the higher dose used in the present study 
suggests that these changes were not sufficient to provoke an acute phase response and hence increase $\alpha_{1}$-AGP levels, although the duration of dosing was shorter here.

Projean et al. (2005) reported that induction of the acute phase response in rats also affected CYP450 expression. A single injection of Freund's complete adjuvant caused a 3-fold increase in plasma $\alpha_{1}$-AGP. Levels of CYP2E1, 2B1/2, 2C11 and total CYP450 levels in rat liver microsomes were significantly reduced 24 and 48 hours after treatment, by $30-77 \%$. Total CYP450 content in liver microsomes inversely correlated significantly with plasma $\alpha_{1}$-AGP levels $(r=-0.60)$. CYP3A2 levels were also reduced, but not significantly so (Projean et al., 2005). This suggests another mechanism by which excipients capable of inducing an acute phase response could affect drug pharmacokinetics.

None of the dosing regimens appeared to affect total protein levels in plasma. The fact that $\alpha_{1}$-AGP makes up such a small amount of total plasma protein means that no increase in total protein concentration was seen in the group where $\alpha_{1}$-AGP levels were increased. Average total protein levels were $58.4 \mathrm{mg} / \mathrm{ml}$ (standard deviation $6.3 \mathrm{mg} / \mathrm{ml}$ ); an increase of $400 \mu \mathrm{g} \alpha_{1}$-AGP (the largest increase observed in the present studies was $385 \mu \mathrm{g} / \mathrm{ml}$ compared to control) would be only $0.7 \%$ of this, and well within the standard 
deviation. It would therefore not be expected to see a significant effect on total plasma protein levels due to such increases $\alpha_{1}$-AGP concentration. However, some of the larger increases in $\alpha_{1}$-AGP concentration reported in the literature (e.g. 15-fold in the work of Sugihara et al.) could potentially result in an increase in total protein levels.

Some of the studies described above, where turpentine was used to induce an acute phase response, report a reduction in albumin levels as well as an increase in $\alpha_{1}$-AGP levels. Matsushima et al. (2000) reported a reduction in albumin levels of around $15 \%$, while Murai-Kuyisha et al. (1993) reported a 30\% reduction. However, Sugihara et al. (1993) reported no significant change in albumin levels in their study. Albumin is present in such high concentrations in plasma that small changes in its concentration would be unlikely to affect drug plasma protein binding. For this reason, we did not measure albumin levels in our study. As albumin constitutes approximately $80 \%$ of total plasma protein in the rat (MuraiKuyisha et al., 1993), large changes in albumin concentration would be expected to be reflected in total protein levels. However, MuraiKuyisha et al. (1993) reported that total plasma protein levels were not significantly affected in their study, despite a $30 \%$ reduction in albumin levels. From their reported albumin levels and total protein levels in the control group ( 34.3 and $42.7 \mathrm{mg} / \mathrm{ml}$, respectively), a 
$24 \%$ reduction in total plasma protein levels would have been expected. The fact that this did not occur suggests that levels of another plasma protein, not measured individually in their study, must have increased.

\subsubsection{Conclusions}

It has been demonstrated that excipients can increase levels of the acute phase reactant $\alpha_{1}$-AGP in rats. However, at lower doses more likely to be used in pre-clinical studies, there was only a single unreproduceable instance of this occurring. It therefore seems unlikely that the excipients tested here would affect drug pharmacokinetics by this mechanism, at doses used in pre-clinical studies.

\subsubsection{Further work}

Further studies could be performed to see if the effect of PG on $\alpha_{1}$ AGP levels reoccurs, and if so to get an idea of the incidence of this effect. However, as this effect did not appear in the second (larger) group of rats tested, it does not appear to be common and may not be due to the excipient at all. The effect of $45 \% 2 \mathrm{ml} / \mathrm{kg}$ DMSO on $\alpha_{1}$-AGP concentrations could also be further investigated. However, 
as this dose of excipient is not routinely used in pre-clinical studies, this would be of limited value.

It would also be of interest to perform longer term multiple dosing studies, where doses of excipient are administered for several days. However, this was not possible in the current study due to license restrictions. 
Chapter 4:

Effects of excipients on P-Gp and ${ }^{99 \mathrm{~m}}$ Tc-MIBI transport in vitro. 


\section{Contents}

4.1 Introduction................................................... 119

4.2 Materials and Methods........................................ 125

4.2.1 Materials ................................................. 125

4.2.2 Methods................................................... 126

4.2.2.1 Cell culture .........................................126

4.2.2.2 Apical to basolateral $(\mathrm{A} \rightarrow \mathrm{B}){ }^{3} \mathrm{H}$-vinblastine and ${ }^{14} \mathrm{C}$ sucrose transport studies........................................ 127

4.2.2.3 Bi-directional ${ }^{99 \mathrm{~m}} \mathrm{TC}-\mathrm{MIBI},{ }^{3} \mathrm{H}$-vinblastine and ${ }^{14} \mathrm{C}$ sucrose transport studies.....................................128

4.2.2.4 Data analysis ......................................... 130

4.3 Results........................................................... 132

4.3.1 $\mathrm{A} \rightarrow \mathrm{B}{ }^{3} \mathrm{H}$-vinblastine and ${ }^{14} \mathrm{C}$-sucrose transport............132

4.3.2 Bi-directional ${ }^{3} \mathrm{H}$-vinblastine and ${ }^{14} \mathrm{C}$-sucrose transport ..140

4.3.3 Bi-directional ${ }^{99 m}$ Tc-MIBI transport .......................143

4.3.4 Bi-directional ${ }^{99 m} \mathrm{Tc}$ transport ...........................147

4.4 Discussion ..................................................... 148

4.4.1 Conclusions ............................................... 160

4.4 .2 Further work .............................................161 


\subsection{Introduction}

There exists in the body a variety of efflux transporters at biological barriers. One of the most widely studied efflux transporters is MDR1 P-glycoprotein (P-Gp), an ATP-binding cassette transporter. P-Gp has a broad substrate specificity, enabling it to transport a wide range of therapeutic drugs, including anticancer compounds, HIV protease inhibitors, immunosupressants and cardiac glycosides (Schinkel and Jonker, 2003). As well as playing a major role in drug resistance of cancer cells, P-Gp has a variety of functions in the body.

Several recent reviews have described the locations of $\mathrm{P}-\mathrm{Gp}$ in various biological barriers, and its roles in the distribution and elimination of its substrates (Ayrton and Morgan, 2001; Lin, 2003; Schinkel and Jonker, 2003; Sun et al., 2004). P-Gp is a principal component of the blood-brain barrier, where it is present in the apical membrane of capillary endothelial cells, and serves to efflux substrates entering the cell back out into the capilliary lumen (Ayrton and Morgan, 2001; Lin, 2003; Sun et al., 2003). In the blood-CSF barrier, P-Gp is present in the apical membrane of choroid plexus cells, where it appears to efflux substrates from the cell back into the CSF (Lin, 2003; Sun et al., 2003). P-Gp in the biliary canalicular membrane of hepatocytes facilitates biliary 
excretion of substrates by efflux into the bile (Ayrton and Morgan, 2001; Lin, 2003). In the apical membrane of renal proximal tubule cells, P-Gp appears to facilitate elimination of substrates in urine by efflux into the renal tubule (Ayrton and Morgan, 2001; Lin, 2003). In the intestine, P-Gp can reduce the oral absorption of its substrates by efflux into the intestinal lumen (Ayrton and Morgan, 2001; Lin, 2003). P-Gp also provides a barrier efflux function at the blood-testes barrier (Schinkel and Jonker, 2003) and the placenta (Lin, 2003).

As would be expected from the locations and barrier efflux function of P-Gp described above, inhibition of this transport protein can have substantial effects on drug distribution and elimination. The literature contains numerous examples of this. For example, Mayer et al. (1997) reported that the P-Gp inhibitor PSC833 significantly affected the distribution and elimination of ${ }^{3} \mathrm{H}$-digoxin in mice. Four hours after injection, plasma levels of ${ }^{3} \mathrm{H}$-digoxin were increased by 2.4-fold, brain levels by 19 -fold, and brain/plasma ratio by 7.8 -fold, demonstrating inhibition of P-Gp mediated efflux in the blood-brain barrier. P-Gp-mediated intestinal efflux of ${ }^{3} \mathrm{H}$-digoxin was also reduced, from $16 \%$ of the administered dose to $1 \%$. There was also a reduction in the biliary elimination of ${ }^{3} \mathrm{H}$-digoxin, from $21 \%$ of the dose to $3 \%$. However, reduced biliary elimination (from $14 \%$ to $4 \%$ ) was also seen when the inhibitor was dosed to mdr1a/1b -/- P-Gp 
knockout mice, suggesting that other transporters may also be involved in this effect. Choo et al. (2000) reported that the P-Gp inhibitor LY-335979 significantly increased levels of ${ }^{3} \mathrm{H}$-nelfinavir in the brain of mice by up to 37 -fold, and levels in the testes by up to 4-fold. Brain/plasma and testes/plasma ratios were also increased, demonstrating that the increased distribution to these organs was due to inhibition of P-Gp mediated efflux from these tissues, not just increased plasma levels. P-Gp inhibition is also the source of several clinically important drug interactions, as discussed by Lin (2003).

Given the potential effects of P-Gp inhibition on drug distribution and elimination, it is important to investigate the effects of excipients on P-Gp, to determine whether they could affect drug disposition by this mechanism. ${ }^{99 \mathrm{~m}} \mathrm{Tc}$-MIBI (Figure 4.1 ) is a diagnostic imaging agent, used clinically for cardiac and oncology applications. Piwnica-Worms et al. (1993) originally reported that ${ }^{99 \mathrm{~m}} \mathrm{Tc}-\mathrm{MIBI}$ is a substrate for P-Gp. Since then, several clinical studies have demonstrated that in vivo ${ }^{99 \mathrm{~m}}$ Tc-MIBI uptake or washout was inversely correlated with P-Gp expression in various tumour types (Kim et al., 1998; Sun et al., 2000; Chang et al., 2003; Kunishio et al., 2003), and in hyperparathyroidism (Yamaguchi et al., 2002). ${ }^{99 \mathrm{~m}} \mathrm{Tc}-\mathrm{MIBI}$ also shows altered disposition 
in mdr1a/b (-/-) P-Gp knockout mice (Dyszlewski et al., 2002), and after dosing with a P-Gp inhibitor (Barbarics et al., 1998).

${ }^{99 m}$ Tc-MIBI was also used in an in vivo model to investigate biliary elimination due to P-Gp and MRP2 (Hendrikse et al., 2004). ${ }^{99 m} \mathrm{Tc}-$ MIBI was used in combination with another agent which is an MRP substrate, so that the differential effects of a given factor or treatment on the biliary elimination of the two compounds could then be used to separate effects due to P-Gp and MRP. This will be discussed in more detail in Chapter 5.

These reports highlight the possibility that ${ }^{99 m}$ Tc-MIBI could be a useful agent to investigate the effects of excipients on $\mathrm{P}-\mathrm{Gp}$ in vivo. Potential advantages of using ${ }^{99 m}$ Tc-MIBI for this purpose, compared with other possible approaches such as determining the pharmacokinetics of P-Gp substrates, include ease of detection, and the potential for non-invasive monitoring of its disposition using gamma scintigraphy.

As mentioned above, ${ }^{99 \mathrm{~m}}$ Tc-MIBI is also a substrate for MRP (Hendrikse et al., 1998; Utsunomiya et al., 2000; Hendrikse et al., 2004). Burak et al. (2003) demonstrated a significant correlation between MRP expression and ${ }^{99 \mathrm{~m}} \mathrm{Tc}-\mathrm{MIBI}$ washout rates in osteosarcoma. However, other authors have reported that MRP 
expression did not significantly correlate with ${ }^{99 \mathrm{~m}}$ Tc-MIBI uptake, despite significant correlations with P-Gp expression (Yamaguchi et al., 2002; Chang et al., 2003). Chang et al. (2003) concluded that ${ }^{99 \mathrm{~m}}$ Tc-MIBI uptake in tumours is more related to P-Gp than MRP expression. It therefore needs to be borne in mind that any excipient induced alterations in ${ }^{99 m}$ Tc-MIBI disposition could be due to MRP, as well as P-Gp. However, as described for the in vivo study by Hendrikse et al. above, it is possible to use study designs where these effects may be separated out to an extent.

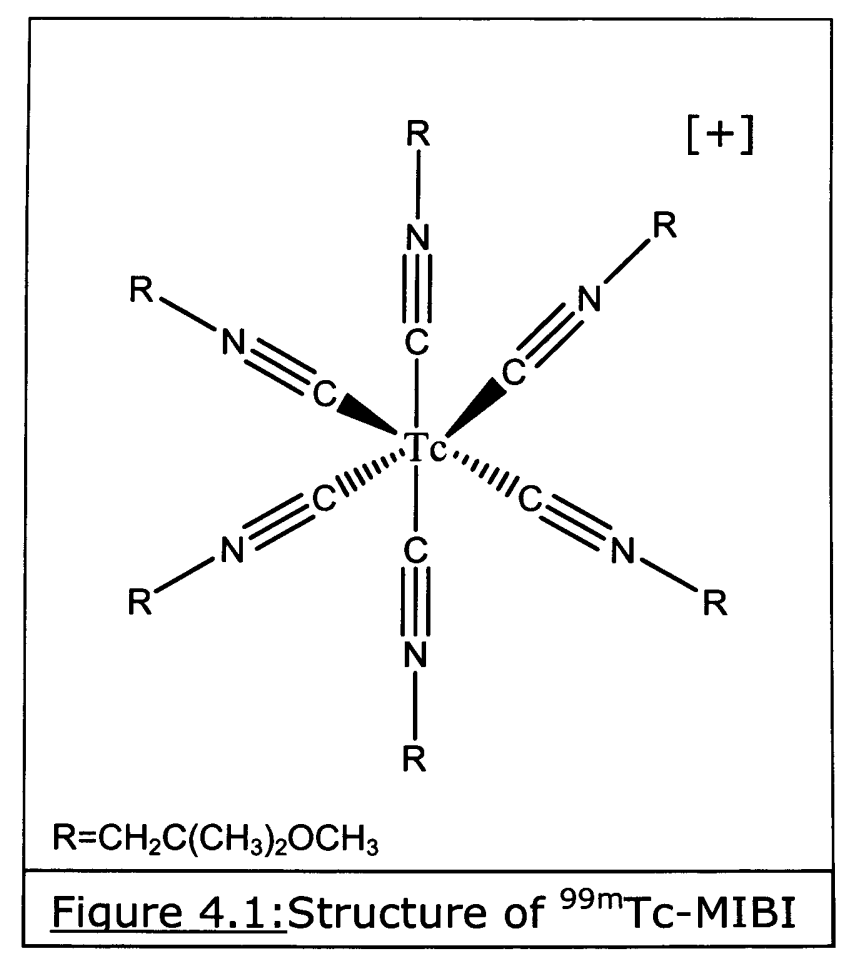

Investigation into P-Gp inhibition has only been reported in the literature for some of the excipients used in formulations for early pharmacokinetic studies. There are reports of $\mathrm{P}-\mathrm{Gp}$ inhibition by Tween 80 and Solutol HS15, but limited reports for PG and PEG 
400 , and no investigations have been reported for DMSO (discussed in Chapter 1). Before proceeding with in vivo studies, it would be useful to determine which excipients can inhibit P-Gp in vitro, and whether they can affect ${ }^{99 \mathrm{~m}} \mathrm{Tc}$-MIBI transport in vitro. This would enable the identification of excipients of interest for use in in vivo ${ }^{99 m}$ Tc-MIBI studies.

The MDCK-MDR1 cell line is a canine kidney epithelial cell line (MDCKII), transfected with human P-Gp. This cell line is widely used to investigate P-Gp in vitro. When grown on permeable supports, these cells form polarised monolayers which overexpress P-Gp on the apical membrane (Borst et al., 1999; Zhang et al., 2003). This makes them a useful in vitro tool for investigating the transport of drug substrates across biological barriers where $\mathrm{P}-\mathrm{Gp}$ is present in vivo. In line with this, MDCK-MDR1 cells have been suggested as a useful in vitro blood-brain barrier permeability screen (Gumbleton and Audus, 2001; Wang et al., 2005), and have also been used to investigate the role of $\mathrm{P}-\mathrm{Gp}$ in the renal elimination of various drugs (Karyekar et al., 2003; Lin, 2003). Hugger et al. (2002) used this cell line to investigate the effects of various excipients on P-Gp (discussed in Chapter 1), and were able to demonstrate a significant inhibitory effect of PEG 300 at a concentration of $20 \%$. This cell line also has the advantages of being easy to culture, and taking a short time for monolayers to become confluent. This model therefore 
appears to be of use for investigating excipient effects on P-Gp in vitro.

This series of experiments aims to use this cell model to identify excipients of interest which can inhibit P-Gp in vitro, by determining the effects of the excipients on the transport of the P-Gp substrate ${ }^{3} \mathrm{H}$-vinblastine, then investigate the effects of these excipients on transport of ${ }^{99 \mathrm{~m}} \mathrm{Tc}-\mathrm{MIBI}$. This will enable the identification of appropriate excipients to use in in vivo studies with ${ }^{99 \mathrm{~m}}$ TC-MIBI.

\subsection{Materials and Methods}

\subsubsection{Materials}

MDCK-MDR1 cells were obtained from Borst (Netherlands). D-MEM, penicillin/streptomycin solution (5000 units/ml penicillin and $5000 \mu \mathrm{g} / \mathrm{ml}$ streptomycin), and foetal bovine serum (FBS), were obtained from Invitrogen, UK. Optiphase HiSafe 3 scintillant, DMSO, Tween 80 , PEG 400, PG, aluminium oxide TLC plates, ethanol, cyclosporin A and Transwell plates and inserts were obtained from Fisher Scientific, UK. Solutol HS15 was a kind gift from AstraZeneca, Alderley Park, UK. ${ }^{3} \mathrm{H}$-vinblastine sulphate and ${ }^{14} \mathrm{C}$ - 
sucrose were obtained from Amersham Biosciences, UK. ${ }^{3} \mathrm{H}-$ vinblastine sulphate had a specific activity of $400 \mathrm{GBq} / \mathrm{mmol}$ $(439 \mathrm{MBq} / \mathrm{mg})$, and a radiochemical purity of $94.2 \% .{ }^{14} \mathrm{C}$-sucrose had a specific activity of $20.9 \mathrm{GBq} / \mathrm{mmol}(58.1 \mathrm{MBq} / \mathrm{mg})$, and a radiochemical purity of $97.8 \%$. The kit for preparation of ${ }^{99 \mathrm{~m}} \mathrm{Tc}$-MIBI (Cardiolite ${ }^{\circledR}$, Bristol-Myers-Squibb) and technetium were obtained from Medical Physics, University Hospital of Wales.

\subsubsection{Methods}

\subsubsection{Cell culture}

MDCK-MDR1 cells (passage no. 5-19) were seeded on Transwell ${ }^{\circledR}$ clear inserts $\left(0.33 \mathrm{~cm}^{2}, 0.4 \mu\right.$ pore diameter $)$ at a density of 50,000 cells $/ \mathrm{cm}^{2}$. Cells were maintained in media consisting of D-MEM with $10 \%$ FBS, penicillin (100units $/ \mathrm{ml})$ and streptomycin $(100 \mu \mathrm{g} / \mathrm{ml})$. Monolayers were grown to confluence before use (day 3-5 post seeding), assessed by visual inspection using a light microscope. 


\subsubsection{Apical to basolateral $(A \rightarrow B){ }^{3} \mathrm{H}$-vinblastine and ${ }^{14} \mathrm{C}$ - sucrose transport studies}

The effects of the excipients on the $A \rightarrow B$ transport of the $P-G p$

substrate ${ }^{3} \mathrm{H}$-vinblastine were assessed at various excipient concentrations, to identify excipients of interest for use in future experiments. Effects of the excipients on transport of ${ }^{14} \mathrm{C}$-sucrose were also investigated, to determine whether any effects seen were due to loss of integrity of the cell monolayer rather than P-Gp inhibition.

Monolayers were inspected for confluence using a light microscope before use. TEER was measured $1-2 \mathrm{~h}$ prior to the studies (EVOM epithelial voltohmeter and EndOhm tissue resistance measurement chamber, World Precision Instruments), and was in the range 50-80 Ohms. $\mathrm{cm}^{2}$.

Monolayers were incubated in serum-free D-MEM for at least 30 minutes prior to the experiment. Media in the apical and basolateral chambers were then changed to serum-free D-MEM containing various concentrations of excipients $(0.1 \%, 1 \%$ or $5 \%)$, and left to equilibrate for 15 minutes before the transport study. To initiate the study, media in the apical chamber was changed for the dosing solution, consisting of serum-free D-MEM containing the excipients 
plus radiolabelled transport substrates $\left(0.4 \mu \mathrm{Ci}{ }^{3} \mathrm{H}\right.$-vinblastine plus $1 \mu \mathrm{Ci}{ }^{14} \mathrm{C}$-sucrose). 6 wells were used for each excipient at each concentration. Parallel controls were run using serum-free D-MEM with no excipients added. During the study, plates were incubated at $37^{\circ} \mathrm{C}$ on an orbital shaker (100rpm). $200 \mu$ l samples were withdrawn from the basolateral chamber at 5, 10, 20, 30, 45, 60 and 80 minutes, with replacement of media. $2.5 \mathrm{ml}$ scintillation cocktail was added to samples prior to counting on a Packard TriCarb 2900TR liquid scintillation counter with internal standard quench correction.

\subsubsection{Bi-directional ${ }^{99 \mathrm{~m}} \mathrm{TC}-\mathrm{MIBI},{ }^{3} \mathrm{H}$-vinblastine and ${ }^{14} \mathrm{C}$ sucrose transport studies}

The effects of $0.1 \%$ Tween 80 and Solutol HS15 on the bidirectional transport of ${ }^{3} \mathrm{H}$-vinblastine, ${ }^{14} \mathrm{C}$-sucrose and ${ }^{99 \mathrm{~m}} \mathrm{Tc}-\mathrm{MIBI}$ were assessed. The P-Gp inhibitor cyclosporin A was also used for comparative purposes.

Monolayers were inspected for confluence using a light microscope before use. Monolayers were incubated in serum-free D-MEM for at least 30 minutes prior to the experiment. Media in the apical and basolateral chambers was then changed to serum-free D-MEM 
containing the P-Gp inhibitors (Tween $800.1 \%$, Solutol HS15 0.1\%, or cyclosporin $\mathrm{A}\left(9.2 \mu \mathrm{M}\right.$ in ${ }^{3} \mathrm{H}$-vinblastine and ${ }^{14} \mathrm{C}$-sucrose studies, $10 \mu \mathrm{M}$ in ${ }^{99 m}$ Tc-MIBI studies)), and left to equilibrate for 15 minutes before the transport study.

${ }^{99 m}$ Tc-MIBI was prepared from a commercially available kit (Cardiolite ${ }^{\circledR}$ ) according to the manufacturers instructions. Radiochemical purity, determined by TLC on aluminium oxide plates as described by the manufacturer, was $95.8 \%$.

To initiate the study, media in the apical or basolateral chamber was changed for the dosing solution, consisting of serum-free DMEM containing the P-Gp inhibitors plus radiolabelled transport substrates $\left(2 \mu \mathrm{Ci} / \mathrm{ml}^{3} \mathrm{H}\right.$-vinblastine plus $12.8 \mu \mathrm{Ci} / \mathrm{ml}^{14} \mathrm{C}$-sucrose, or ${ }^{99 \mathrm{~m}} \mathrm{Tc}-\mathrm{MIBI}(0.1 \mathrm{MBq} / \mathrm{ml}$ at $\left.12 \mathrm{pm})\right)$. Transport of free ${ }^{99 \mathrm{~m}} \mathrm{Tc}$ $(0.1 \mathrm{MBq} / \mathrm{ml}$ at $12 \mathrm{pm})$ was also investigated. 5-6 wells were used for each treatment. Parallel controls were run using serum-free D-MEM with no excipients added.

During the ${ }^{3} \mathrm{H}$-vinblastine and ${ }^{14} \mathrm{C}$-sucrose transport study, plates were incubated at $37^{\circ} \mathrm{C}$ on an orbital shaker (100rpm). During the ${ }^{99 \mathrm{~m}} \mathrm{Tc}-$ MIBI study, plates were incubated at $37^{\circ} \mathrm{C}$ in a Stuart Scientific SI2OH hybridization oven/shaker, with shaking at 5 strokes/min. Samples were withdrawn from the apical $(100 \mu l)$ or 
basolateral $(200 \mu l)$ chambers at $5,10,20,30,45,60$ and 80 minutes, with replacement of media.

Samples from the ${ }^{3} \mathrm{H}$-vinblastine and ${ }^{14} \mathrm{C}$-sucrose transport study were counted on a Packard TriCarb 2900TR liquid scintillation counter as described above. Samples from the ${ }^{99 m}$ Tc-MIBI transport experiments were counted on a Packard Cobra II gamma counter, with half-life correction (counts were also corrected for the time of day the image was taken to enable comparison).

\subsubsection{Data analysis}

Background counts were subtracted from the counts obtained for each sample. The permeability coefficients $(\rho)$ of the radiolabelled transport substrates across the monolayers were calculated according to the equation $\mathrm{dM} / \mathrm{dt}=\rho \times \mathrm{A} \times \mathrm{C}_{0}$, where $\mathrm{A}$ is the surface area of the monolayer, $C_{0}$ is the initial substrate concentration in the donor chamber (assumed to remain essentially constant throughout the experiment) and $\mathrm{dM} / \mathrm{dt}$ is the cumulative rate of transport into the receiver chamber (assessed by the gradient of a cumulative counts vs. time curve). 
Counted aliquots of the dosing solution were used to determine accurate initial concentrations of transport substrates for use in calculations.

Significance was assessed using a one-way ANOVA with Bonferroni's post-hoc test. Homogeneity of variance was assessed using Levene's test. Where variances were heterogeneous, significance was assessed using the Games-Howell test (Sokal and Rohlf, 1995); instances where this has been undertaken are highlighted in the results section. 


\subsection{Results}

\subsection{1 $A \rightarrow B{ }^{3} \mathrm{H}$-vinblastine and ${ }^{14} \mathrm{C}$-sucrose transport}

Table 4.1 shows the influence of $1 \%$ of the excipients on the apicalto-basolateral $(A \rightarrow B)$ permeability coefficients of ${ }^{3} \mathrm{H}$-vinblastine and ${ }^{14} \mathrm{C}$-sucrose across MDCK-MDR1 monolayers.

Table 4.1: $A \rightarrow B$ permeability coefficients of ${ }^{3} \mathrm{H}$-vinblastine and ${ }^{14} \mathrm{C}$ sucrose across MDCK-MDR1 monolayers, in the presence of $1 \%$ excipients.

\begin{tabular}{|c|c|c|c|c|c|c|c|c|}
\hline & \multicolumn{7}{|c|}{ Permeability coefficient $\left(\mathrm{cm} \mathrm{sec}^{-1}, \times 10^{-6}\right)$} \\
\hline & & 1 & 2 & 3 & 4 & 5 & 6 & Mean ( \pm SD) \\
\hline \multirow[t]{2}{*}{ Control } & $\mathrm{s}$ & 0.29 & 0.31 & 0.26 & 0.23 & 0.46 & 0.26 & $0.30 \quad( \pm 0.08)$ \\
\hline & v & 0.15 & 0.19 & 0.26 & 0.17 & 0.19 & 0.17 & $0.19 \quad( \pm 0.04)$ \\
\hline \multirow[t]{2}{*}{ DMSO } & $\mathrm{S}$ & & 0.41 & 0.92 & 0.41 & & 0.42 & $0.51( \pm 0.21)$ \\
\hline & $\mathrm{v}$ & 0.12 & 0.14 & 0.31 & 0.15 & 0.19 & 0.19 & $0.18 \quad( \pm 0.07)$ \\
\hline \multirow{2}{*}{$\begin{array}{c}\text { Tween } \\
80 \\
\end{array}$} & $\mathrm{~S}$ & 0.77 & 1.8 & 0.81 & 0.71 & 0.80 & 0.94 & $0.98 \quad( \pm 0.43)$ \\
\hline & $\mathrm{v}$ & 0.83 & 1.08 & 0.68 & 0.73 & 0.74 & 1.31 & $0.89 \quad( \pm 0.25)$ \\
\hline \multirow{2}{*}{$\begin{array}{l}\text { PEG } \\
400\end{array}$} & $\mathrm{~s}$ & 0.28 & 0.19 & 0.24 & 0.19 & 0.27 & & $0.23 \quad( \pm 0$. \\
\hline & $\mathrm{V}$ & 0.38 & 0.35 & 0.33 & 0.32 & 0.37 & & $0.35 \quad( \pm 0.02)$ \\
\hline \multirow[t]{2}{*}{ PG } & $\mathrm{S}$ & 0.65 & 0.73 & 0.31 & 0.42 & 0.4 & 0.60 & $( \pm 0.17)$ \\
\hline & $\mathrm{v}$ & 0.36 & 0.32 & 0.24 & 0.29 & 0.28 & 0.34 & $( \pm 0.04)$ \\
\hline \multirow{2}{*}{$\begin{array}{c}\text { Solutol } \\
\text { HS15 }\end{array}$} & $\mathrm{s}$ & 0.75 & 0.76 & & & & 0.74 & $( \pm 0.14)$ \\
\hline & v & 0.73 & 0.73 & 0.73 & 0.75 & 0.68 & 0.77 & $0.73( \pm 0.03)$ \\
\hline
\end{tabular}

$\mathrm{S}=$ sucrose, $\mathrm{V}=$ vinblastine

Figures 4.2 and 4.3 show which of these permeability coefficients were significantly different from each other for ${ }^{14} \mathrm{C}$-sucrose and ${ }^{3} \mathrm{H}$ vinblastine respectively. As variances for the ${ }^{3} \mathrm{H}$-vinblastine data 
were heterogeneous, significance was assessed using the GamesHowell test.
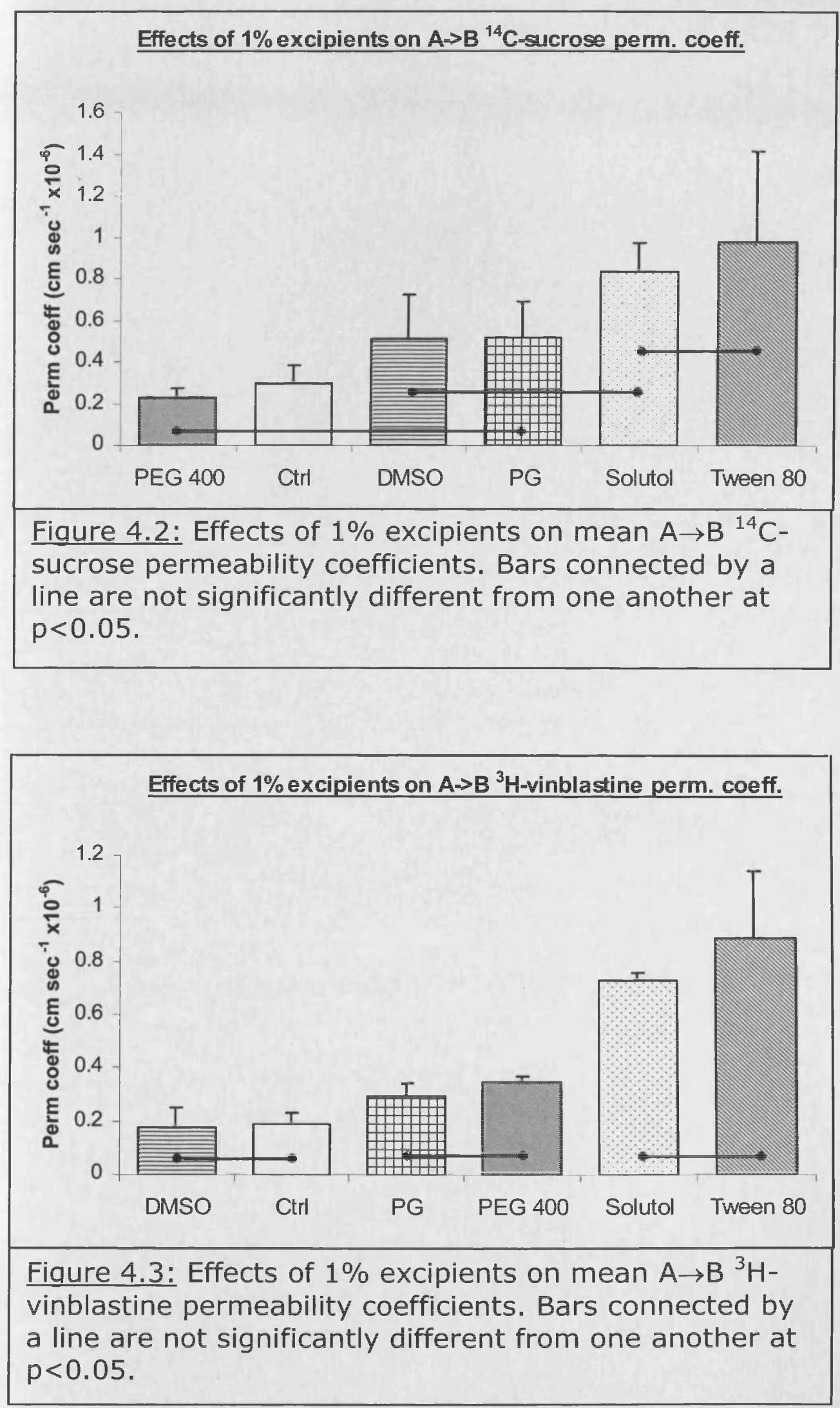
Table 4.2 shows the effects of a $0.1 \%$ concentration of the excipients on the $A \rightarrow B$ permeability coefficients of ${ }^{3} \mathrm{H}$-vinblastine and ${ }^{14} \mathrm{C}$-sucrose across the monolayers.

Table 4.2: $A \rightarrow B$ permeability coefficients of ${ }^{3} \mathrm{H}$-vinblastine and ${ }^{14} \mathrm{C}$ sucrose across MDCK-MDR1 monolayers, in the presence of $0.1 \%$ excipients.

\begin{tabular}{|c|c|c|c|c|c|c|c|c|}
\hline & \multicolumn{7}{|c|}{ Permeability coefficient $\left(\mathrm{cm} \mathrm{sec}^{-1}, \times 10^{-6}\right)$} \\
\hline & & 1 & 2 & 3 & 4 & 5 & 6 & Mean ( \pm SD) \\
\hline \multirow[t]{2}{*}{ Control } & $\mathrm{S}$ & 0.72 & 0.66 & 0.99 & 0.73 & 0.47 & 0.35 & $0.65 \quad( \pm 0.22)$ \\
\hline & $\mathrm{v}$ & 0.09 & 0.06 & 0.00 & 0.15 & 0.06 & 0.11 & $0.08 \quad( \pm 0.05)$ \\
\hline \multirow[t]{2}{*}{ DMSO } & $\mathrm{S}$ & 0.37 & 0.34 & 0.53 & 0.71 & 0.67 & 0.84 & $( \pm 0.20)$ \\
\hline & $\mathrm{v}$ & 0.05 & 0.05 & 0.07 & 0.04 & 0.05 & 0.06 & $( \pm 0.01)$ \\
\hline Tween & $\mathrm{S}$ & 0.22 & 0.74 & 0.27 & 0.82 & 0.67 & 1.26 & $( \pm 0.38)$ \\
\hline 80 & $\mathrm{v}$ & 0.49 & 0.74 & 0.60 & 0.75 & 0.66 & 0.81 & $( \pm 0.12)$ \\
\hline PEG & $\mathrm{s}$ & 0.65 & 0.75 & 0.32 & 1.08 & 0.63 & 0.53 & $( \pm 0.25)$ \\
\hline 400 & V & 0.08 & 0.03 & 0.12 & 0.03 & 0.12 & 0.15 & $( \pm 0.05)$ \\
\hline \multirow[t]{2}{*}{ PG } & 5 & 1.06 & 0.88 & 0.53 & 0.64 & 1.02 & 1.02 & $( \pm 0.22)$ \\
\hline & V & 0.03 & 0.08 & 0.07 & 0.07 & 0.05 & 0.07 & $( \pm 0.02)$ \\
\hline Solutol & $S$ & 0.79 & 0.78 & 0.61 & 0.73 & 0.48 & 0.65 & $( \pm 0.12)$ \\
\hline HS15 & v & 1.09 & 1.18 & 1.10 & 1.18 & 1.12 & 1.09 & $( \pm 0.04)$ \\
\hline
\end{tabular}

$\mathrm{S}=$ sucrose, $\mathrm{V}=$ vinblastine

There was no significant difference in mean ${ }^{14} \mathrm{C}$-sucrose permeability coefficient between any of the groups. Figure 4.4 shows significant differences in mean ${ }^{3} \mathrm{H}$-vinblastine permeability coefficients between groups. As variances for the ${ }^{3} \mathrm{H}$-vinblastine data were heterogeneous, significance was assessed using the Games-Howell test. 


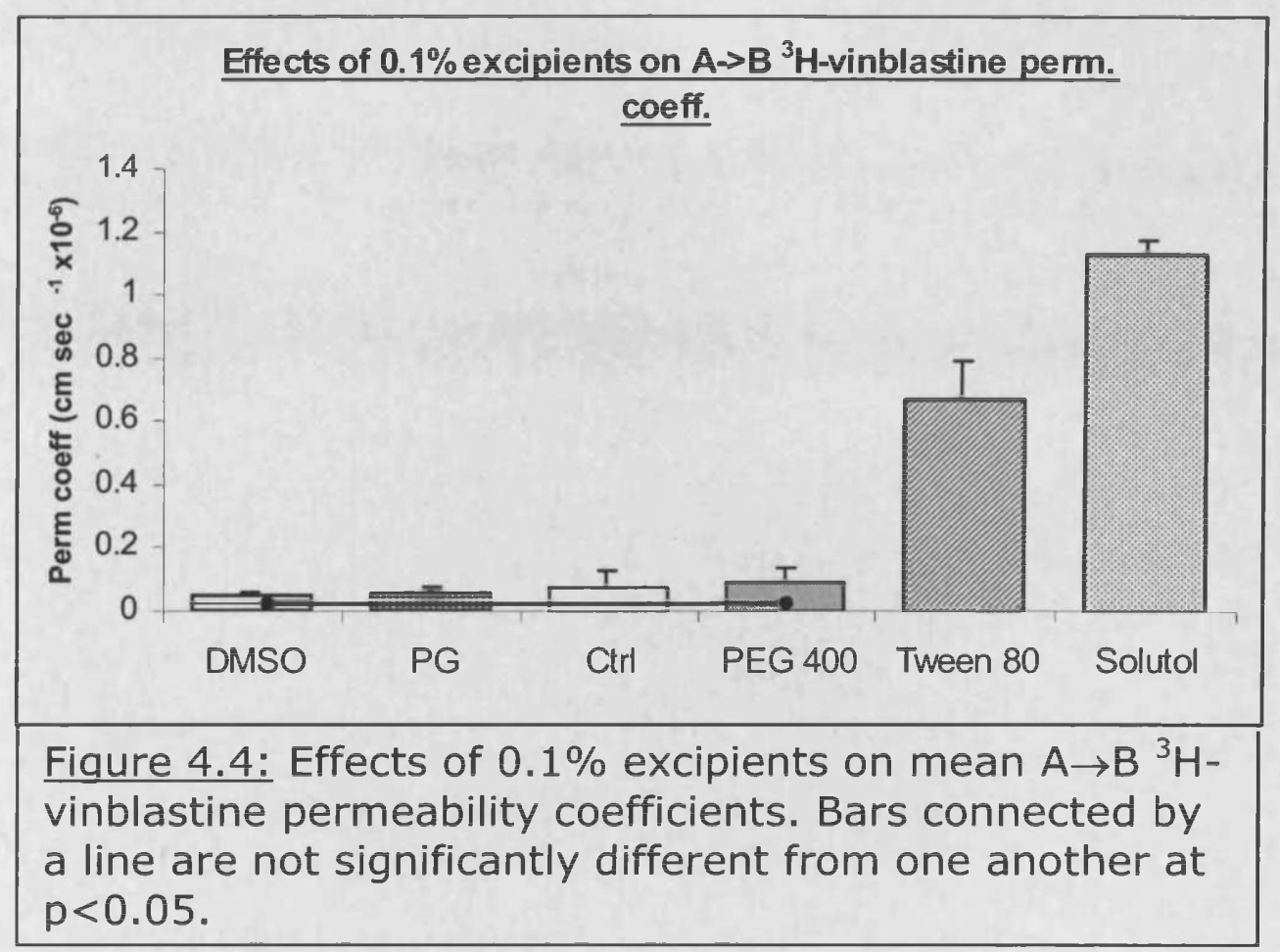

In the experiments reported above, it appeared that $0.1 \%$ Tween 80 and Solutol HS15 had a greater inhibitory effect on P-Gp than a concentration of $1 \%$ (4.7 and 3.8 fold increases in ${ }^{3} \mathrm{H}$-vinblastine permeability at $1 \%$, versus 8 and 14 -fold increases at $0.1 \%$, for Tween and Solutol respectively). However, there was not such a marked difference in the absolute permeability coefficients. The permeability coefficients for Solutol were $0.73 \mathrm{~cm} \mathrm{sec}^{-1} \times 10^{-6}$ at $1 \%$ and $1.13 \mathrm{~cm} \mathrm{sec}^{-1} \times 10^{-6}$ at $0.1 \%$. For Tween, the absolute permeability coefficient was greater at a concentration of $1 \%(0.89$ $\mathrm{cm} \mathrm{sec}{ }^{-1} \times 10^{-6}$, vs. $0.67 \mathrm{~cm} \mathrm{sec}^{-1} \times 10^{-6}$ at $0.1 \%$ ). The apparent difference in their potencies at different concentrations may therefore be due to the differences in vinblastine permeability 
coefficient across the control monolayers in the different batches of cells used for the two experiments $\left(0.19 \mathrm{~cm} \mathrm{sec}^{-1} \times 10^{-6}\right.$ for the $1 \%$ excipients experiment, vs. $0.08 \mathrm{~cm} \mathrm{sec}^{-1} \times 10^{-6}$ for the $0.1 \%$ experiment). In order to clarify this, these experiments were repeated to run both $1 \%$ and $0.1 \%$ excipient concentrations at the same time in the same batch of cells. Results are shown in Table 4.3. It can be seen that the difference in magnitude between the effects seen at different concentrations has decreased. Solutol caused a 3 -fold increase at $0.1 \%$ and a 2.2 -fold increase at $1 \%$, while Tween caused a 2.7 -fold increase at $0.1 \%$ and a 2.8 -fold increase at $1 \%$. This variation in the magnitude of effect seen on ${ }^{3} \mathrm{H}$-vinblastine transport at different concentrations in the above data therefore appears to be due to between-batch variations between cells. 
Table 4.3: $\mathrm{A} \rightarrow \mathrm{B}$ permeability coefficients of ${ }^{3} \mathrm{H}$-vinblastine and ${ }^{14} \mathrm{C}$ sucrose across MDCK-MDR1 monolayers, in the presence of $1 \%$ and $0.1 \%$ Tween 80 and Solutol HS15.

\begin{tabular}{|c|c|c|c|c|c|c|c|c|c|}
\hline & \multicolumn{8}{|c|}{ Permeability coefficient $\left(\mathrm{cm} \mathrm{sec}^{-1}, \times_{10}^{-6}\right)$} \\
\hline & & 1 & 2 & 3 & 4 & 5 & 6 & Mea & $( \pm S D)$ \\
\hline \multirow[t]{2}{*}{ Control } & $\mathrm{S}$ & 0.62 & & & & & & 0.65 & \\
\hline & V & & & & & & & 0.28 & \\
\hline \multirow{2}{*}{$\begin{array}{c}\text { Tween } \\
1 \%\end{array}$} & $\mathrm{~S}$ & & & & & & & & \\
\hline & V & & & & & & & & \\
\hline \multirow{2}{*}{$\begin{array}{c}\text { Tween } \\
0.1 \%\end{array}$} & $\mathrm{~S}$ & & & & & & & & \\
\hline & $\mathrm{V}$ & & & & & & & & \\
\hline \multirow{2}{*}{$\begin{array}{c}\text { Solutol } \\
1 \% \\
\end{array}$} & $S$ & & & & & & & 1.20 & \\
\hline & V & & & & & & & 0.62 & \\
\hline \multirow{2}{*}{$\begin{array}{c}\text { Solutol } \\
0.1 \%\end{array}$} & $\mathrm{~S}$ & & & & & & & & \\
\hline & V & 0.69 & 0.96 & 0.85 & 0.71 & 0.81 & 1.15 & 0.86 & $( \pm 0.17$ \\
\hline
\end{tabular}

$\mathrm{S}=$ sucrose, $\mathrm{V}=$ vinblastine

Figures 4.5 and 4.6 show which of the mean ${ }^{14} \mathrm{C}$-sucrose and ${ }^{3} \mathrm{H}$ vinblastine permeability coefficients were significantly different from each other. 

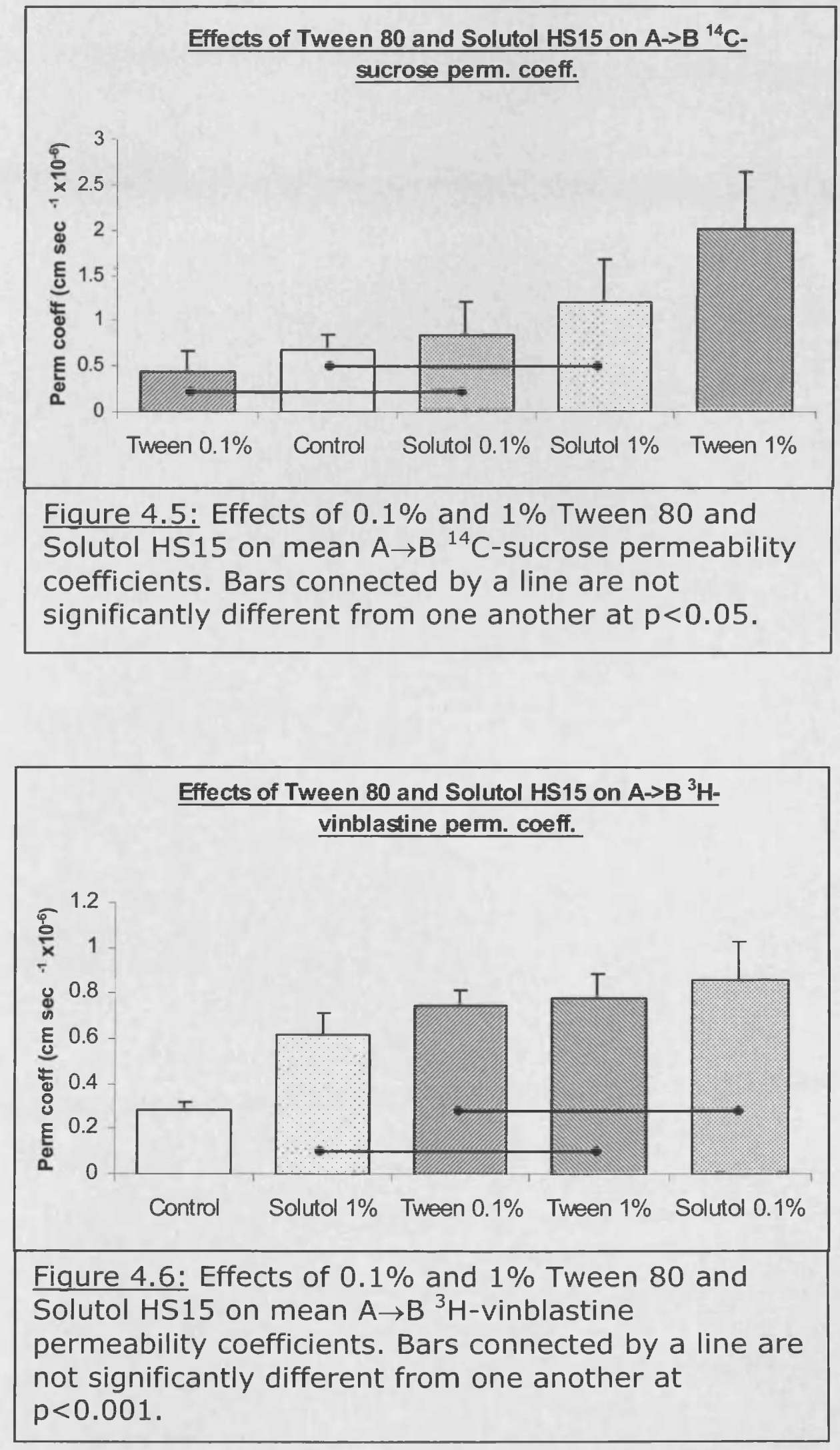
As the magnitude of effect seen with $1 \%$ PG was small, this experiment was repeated, using PG concentrations of $1 \%$ and $5 \%$, to clarify that an effect had been seen. Results are shown in Table 4.4 .

Table 4.4: $A \rightarrow B$ permeability coefficients of ${ }^{3} \mathrm{H}$-vinblastine and ${ }^{14} \mathrm{C}$ sucrose across MDCK-MDR1 monolayers, in the presence of $1 \%$ and $5 \%$ PG.

\begin{tabular}{|c|c|c|c|c|c|c|c|c|}
\hline & \multicolumn{7}{|c|}{ Permeability coefficient $\left(\mathrm{cm} \mathrm{sec}{ }^{-1}, x^{-6}\right)$} \\
\hline & & 1 & 2 & 3 & 4 & 5 & 6 & Mean $( \pm$ SD $)$ \\
\hline \multirow[t]{2}{*}{ Control } & $\mathrm{s}$ & 0.88 & 0.89 & 0.56 & 1.86 & 0.61 & 0.72 & $0.92( \pm 0.48)$ \\
\hline & $\mathrm{V}$ & 0.59 & 0.68 & 0.43 & 0.95 & 0.46 & 0.67 & $( \pm 0.19)$ \\
\hline \multirow[t]{2}{*}{ PG 1\% } & $\mathrm{S}$ & 0.78 & 0.89 & 1.41 & 0.82 & 0.79 & 0.71 & $( \pm 0.25)$ \\
\hline & v & 0.44 & 0.56 & 0.37 & 0.61 & 0.52 & 0.42 & $( \pm 0.09)$ \\
\hline \multirow[t]{2}{*}{ PG 5\% } & $\mathrm{S}$ & 0.92 & 1.13 & 1.39 & 0.98 & 0.91 & 1.37 & $( \pm 0.22)$ \\
\hline & $\mathrm{v}$ & 0.75 & 0.74 & 0.81 & 0.77 & 0.59 & 1.11 & $( \pm 0.17)$ \\
\hline
\end{tabular}

$\mathrm{S}=$ sucrose, $\mathrm{V}=$ vinblastine

There was no significant difference in mean ${ }^{14} \mathrm{C}$-sucrose

permeability coefficient between any of the groups in this experiment. Figure 4.7 shows which of the mean ${ }^{3} \mathrm{H}$-vinblastine permeability coefficients were significantly different from each other. 


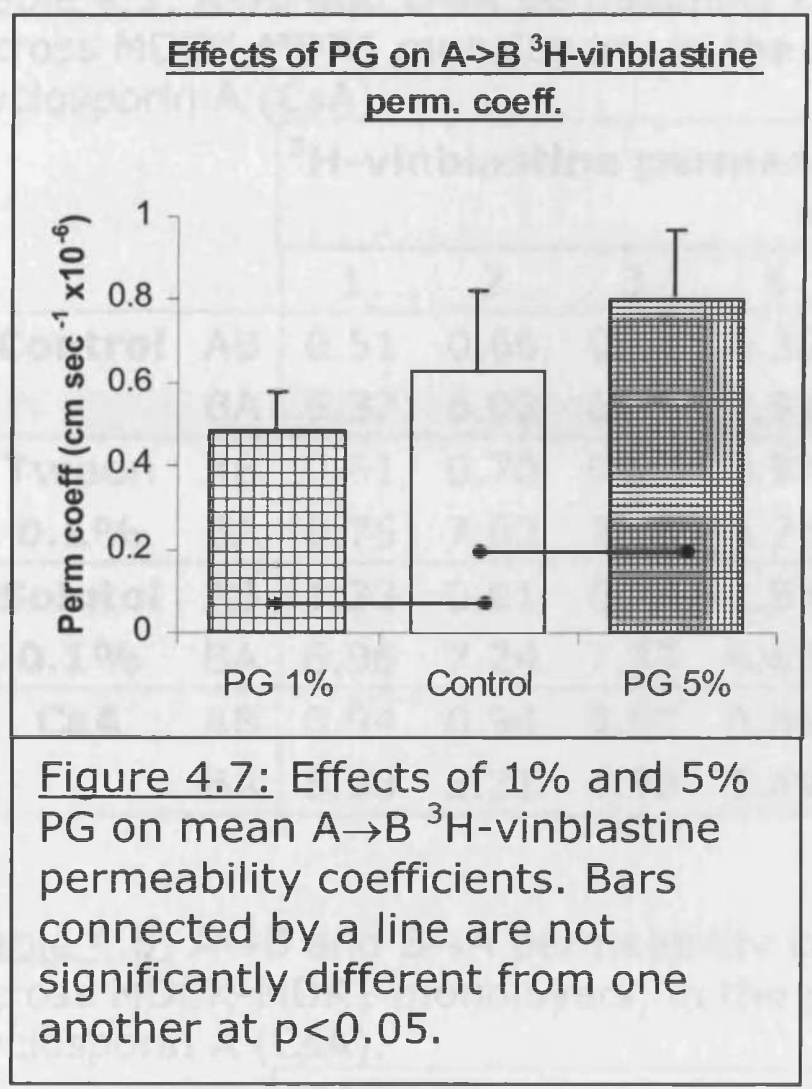

\subsubsection{Bi-directional ${ }^{3} \mathrm{H}$-vinblastine and ${ }^{14} \mathrm{C}$-sucrose transport}

The effects of the P-Gp inhibitors on bi-directional transport of ${ }^{3} \mathrm{H}$ -

vinblastine and ${ }^{14} \mathrm{C}$-sucrose are shown in Tables 4.5 and 4.6, respectively. Unfortunately, due to a pipetting error, the concentration of cyclosporin A used in these experiments was $9.2 \mu \mathrm{M}$, slightly lower than the $10 \mu \mathrm{M}$ concentration intended. 
Table 4.5: $A \rightarrow B$ and $B \rightarrow A$ permeability coefficients of ${ }^{3} \mathrm{H}$-vinblastine across MDCK-MDR1 monolayers, in the presence of excipients and cyclosporin A (CSA).

\begin{tabular}{|c|c|c|c|c|c|c|c|c|}
\hline & & \multicolumn{7}{|c|}{$\begin{array}{c}{ }^{3} \mathrm{H} \text {-vinblastine permeability coefficient }\left(\mathrm{cm} \mathrm{sec}^{-1}\right. \\
\left.\times 10^{-6}\right)\end{array}$} \\
\hline & & 1 & 2 & 3 & 4 & 5 & 6 & Mean ( \pm SD) \\
\hline \multirow[t]{2}{*}{ Control } & $A B$ & 0.51 & 0.60 & 0.51 & 0.35 & 0.31 & & $0.46( \pm 0.12)$ \\
\hline & $\mathrm{BA}$ & 6.37 & 6.03 & 6.50 & 6.91 & 7.21 & & $6.60( \pm 0.46)$ \\
\hline \multirow{2}{*}{$\begin{array}{l}\text { Tween } \\
0.1 \%\end{array}$} & $A B$ & 0.61 & 0.70 & 0.62 & 0.91 & 0.61 & 0.57 & $0.67( \pm 0.13)$ \\
\hline & BA & 6.75 & 7.02 & 7.10 & 6.71 & 7.20 & & $6.95( \pm 0.22)$ \\
\hline \multirow{2}{*}{$\begin{array}{l}\text { Solutol } \\
0.1 \%\end{array}$} & $A B$ & 1.33 & 0.91 & 0.78 & 1.53 & 0.90 & 0.75 & $1.03( \pm 0.32)$ \\
\hline & BA & 6.96 & 7.24 & 7.37 & 6.63 & 6.71 & & $6.98( \pm 0.32)$ \\
\hline \multirow[t]{2}{*}{ CsA } & $A B$ & 0.94 & 0.94 & 0.87 & 0.84 & 1.00 & 0.86 & $0.91( \pm 0.06)$ \\
\hline & BA & 5.53 & 5.31 & 4.90 & 5.49 & 5.57 & & $5.36( \pm 0.27)$ \\
\hline
\end{tabular}

Table 4.6: $A \rightarrow B$ and $B \rightarrow A$ permeability coefficients of ${ }^{14} \mathrm{C}$-sucrose across MDCK-MDR1 monolayers, in the presence of excipients and cyclosporin A (CsA).

\begin{tabular}{|c|c|c|c|c|c|c|c|c|}
\hline & \multicolumn{7}{|c|}{$\begin{array}{l}{ }^{14} \mathrm{C} \text {-sucrose permeability coefficient }\left(\mathrm{cm} \mathrm{sec}^{-1},\right. \\
\qquad\left(10^{-6}\right)\end{array}$} \\
\hline & & 1 & 2 & 3 & 4 & 5 & 6 & Mean ( \pm SD) \\
\hline \multirow[t]{2}{*}{ Control } & $A B$ & 0.82 & 1.15 & 0.63 & 0.31 & 0.20 & & $0.62( \pm 0.38)$ \\
\hline & BA & 0.32 & 0.40 & 0.43 & 0.31 & 0.31 & & $0.35( \pm 0.06)$ \\
\hline \multirow{2}{*}{$\begin{array}{l}\text { Tween } \\
0.1 \% \\
\end{array}$} & $A B$ & 0.18 & 0.23 & 0.18 & 0.86 & 0.18 & 0.16 & $0.30( \pm 0.28)$ \\
\hline & BA & 0.35 & 0.99 & 0.44 & 0.40 & 0.60 & & $0.55( \pm 0.26)$ \\
\hline \multirow{2}{*}{$\begin{array}{c}\text { Solutol } \\
0.1 \%\end{array}$} & $A B$ & 1.24 & 0.42 & 0.35 & 1.24 & 0.53 & 0.23 & $0.67( \pm 0.45)$ \\
\hline & $\mathrm{BA}$ & 0.37 & 0.40 & 0.40 & 0.50 & 0.40 & & $0.41( \pm 0.05)$ \\
\hline \multirow[t]{2}{*}{ CsA } & $A B$ & 0.35 & 0.28 & 0.22 & 0.19 & 0.35 & 0.19 & $0.26( \pm 0.08)$ \\
\hline & BA & 1.58 & 1.14 & 0.47 & 0.98 & 0.36 & & $0.91( \pm 0.50)$ \\
\hline
\end{tabular}

The ${ }^{14} \mathrm{C}$-sucrose permeabilities of the cell monolayers these experiments were very variable in some of the groups (e.g. $A \rightarrow B$ permeability coefficient of ${ }^{14} \mathrm{C}$-sucrose in control group $0.62 \pm 0.38$ $\left.\mathrm{cm} / \mathrm{sec} \times 10^{-6}\right)$. All of the monolayers were confluent when inspected. However, there were no significant differences in ${ }^{14} \mathrm{C}$ - 
sucrose permeabilities between groups, demonstrating that none of the treatments significantly affected the permeability of the monolayer. As variances in the ${ }^{14} \mathrm{C}$-sucrose data were heterogeneous according to Levene's test, the Games-Howell test was used to assess significance.

Figures 4.7 and 4.8 show significant differences in mean ${ }^{3} \mathrm{H}$ vinblastine permeability coefficients between groups in the $A \rightarrow B$ and $B \rightarrow A$ directions, respectively. As variances in the $A \rightarrow B$ data were heterogeneous according to Levene's test, the Games-Howell test was used to assess significance. BA/AB ratios were 14.3 for control, 10.4 for Tween 80, 6.8 for Solutol and 5.9 for cyclosporin A.

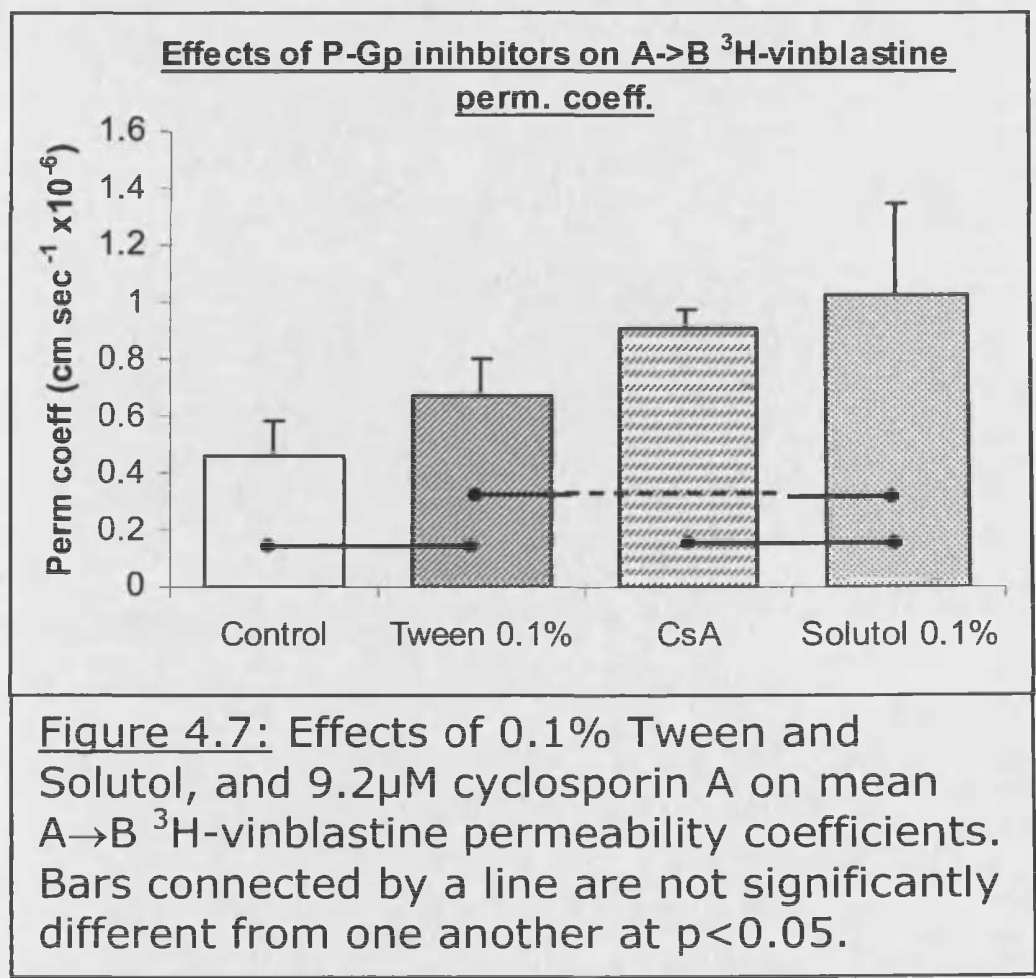




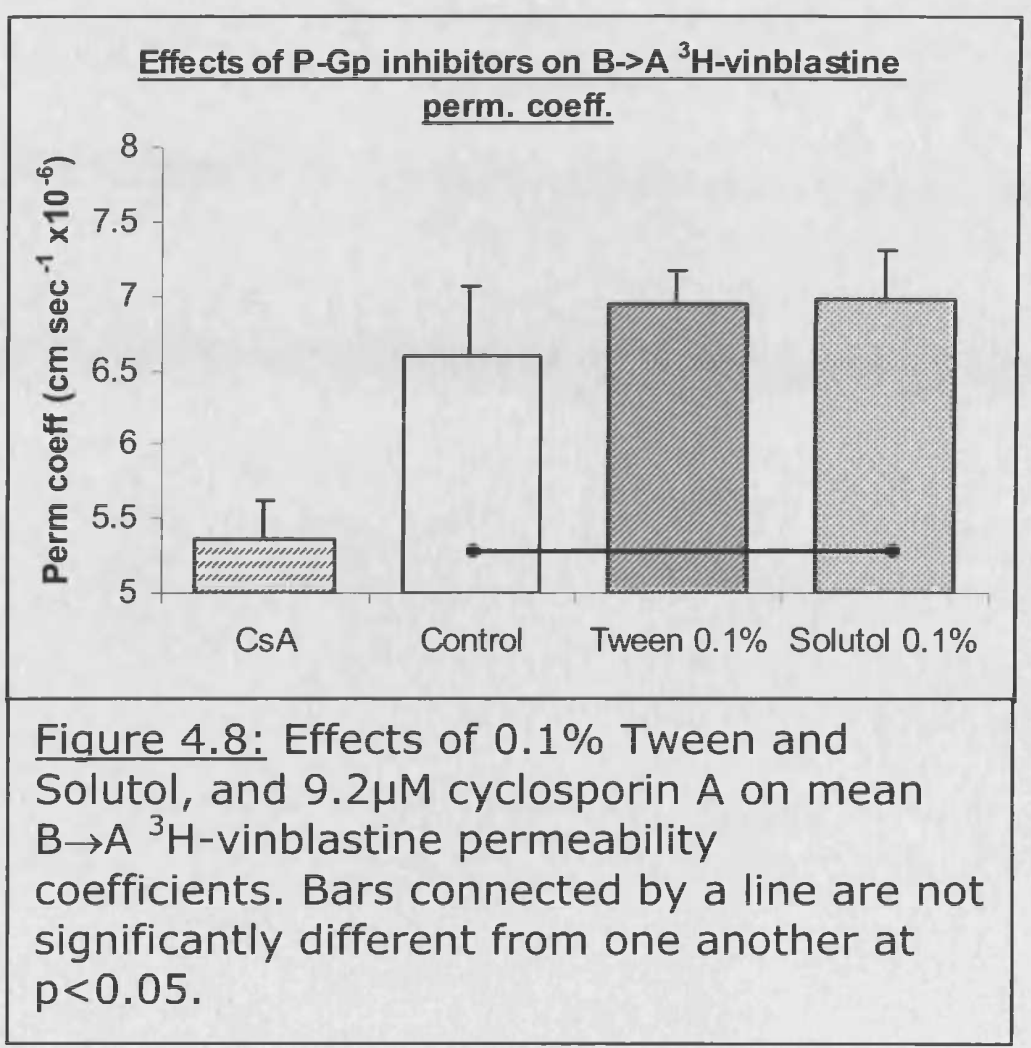

\subsubsection{Bi-directional ${ }^{99 \mathrm{~m}}$ TC-MIBI transport}

In most of the monolayers, a lag time was apparent before detectable quantities of ${ }^{99 \mathrm{~m}} \mathrm{Tc}$-MIBI were transported to the other side of the monolayer. This was usually between 10 and 30 minutes long, although two of the monolayers exhibited lag times of an hour. Permeability coefficients were calculated from the gradient of the straight part of the graph, after the lag time had elapsed.

Permeability coefficients of ${ }^{99 \mathrm{~m}} \mathrm{Tc}-\mathrm{MIBI}$ across the monolayers in both directions are shown in Table 4.7. 
Table 4.7: $A \rightarrow B$ and $B \rightarrow A$ permeability coefficients of ${ }^{99 m}$ Tc-MIBI across MDCK-MDR1 monolayers, in the presence of excipients and cyclosporin A (CsA).

\begin{tabular}{|c|c|c|c|c|c|c|c|c|}
\hline & \multicolumn{7}{|c|}{$\begin{array}{l}{ }^{99 \mathrm{~m}} \text { Tc-MIBI permeability coefficient }\left(\mathrm{cm} \mathrm{sec}^{-1},\right. \\
\times\left(10^{-6}\right)\end{array}$} \\
\hline & & 1 & 2 & 3 & 4 & 5 & 6 & Mean ( \pm SD) \\
\hline \multirow[t]{2}{*}{ Control } & $A B$ & 0.02 & 0.04 & 0.03 & 0.13 & 0.03 & & $0.05 \quad( \pm 0.04)$ \\
\hline & BA & 3.04 & 3.12 & 3.78 & 3.51 & 3.21 & 4.04 & $3.45( \pm 0.40)$ \\
\hline \multirow{2}{*}{$\begin{array}{l}\text { Tween } \\
0.1 \%\end{array}$} & $A B$ & 0.15 & 0.10 & 0.27 & 0.40 & 0.25 & 0.14 & $0.22( \pm 0.11)$ \\
\hline & BA & 14.78 & 17.09 & 15.36 & 15.55 & 16.82 & 13.25 & $15.47( \pm 1.41)$ \\
\hline \multirow{2}{*}{$\begin{array}{l}\text { Solutol } \\
0.1 \%\end{array}$} & $\overline{A B}$ & 0.20 & 0.21 & 0.22 & 0.52 & 0.30 & & $0.29( \pm 0.13)$ \\
\hline & BA & 13.62 & 13.66 & 15.20 & 12.55 & 13.60 & & $13.72( \pm 0.95)$ \\
\hline \multirow[t]{2}{*}{ CsA } & $A B$ & 0.25 & 0.24 & 0.16 & 0.36 & 0.11 & 0.11 & $0.20 \quad( \pm 0.10)$ \\
\hline & BA & 2.43 & 4.29 & 3.74 & 5.66 & 4.18 & 4.38 & $4.11 \quad( \pm 1.05)$ \\
\hline
\end{tabular}

Figures 4.9 and 4.10 show significant differences in mean ${ }^{99 \mathrm{~m}} \mathrm{Tc}$ MIBI permeability coefficients between groups in the $A \rightarrow B$ and $B \rightarrow A$ directions, respectively. $B A / A B$ ratios were 69.0 for control, 70.3 for Tween, 47.3 for Solutol and 20.6 for cyclosporin $A$. 

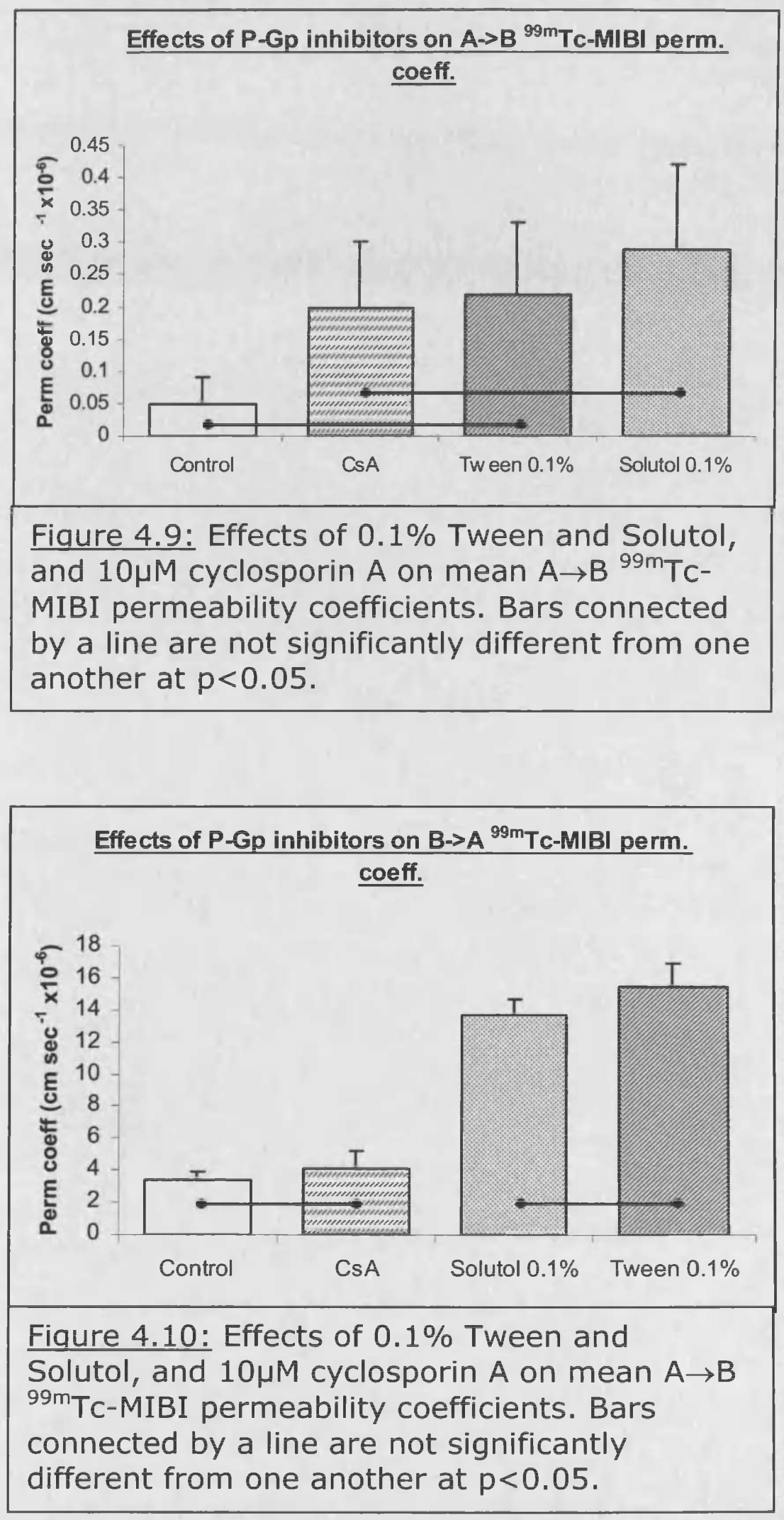
As described above, the lag time in the ${ }^{99 m}$ Tc-MIBI data made determination of the permeability coefficients less straightforward. It was therefore decided to employ a second method of analysis for this data, to ensure that the results were not dependent on our judgement of when the lag time had elapsed. The bi-directional ${ }^{99 m}$ Tc-MIBI data were therefore also analysed in terms of cumulative counts per minute (CPM) transported over the whole 80 minute sampling period. Figures 4.11 and 4.12 show the effects of the excipients and cyclosporin A on cumulative ${ }^{99 \mathrm{~m}} \mathrm{Tc}$-MIBI transport in the $A \rightarrow B$ and $B \rightarrow A$ directions. It can be seen that the data are in good agreement with the permeability coefficient data. 

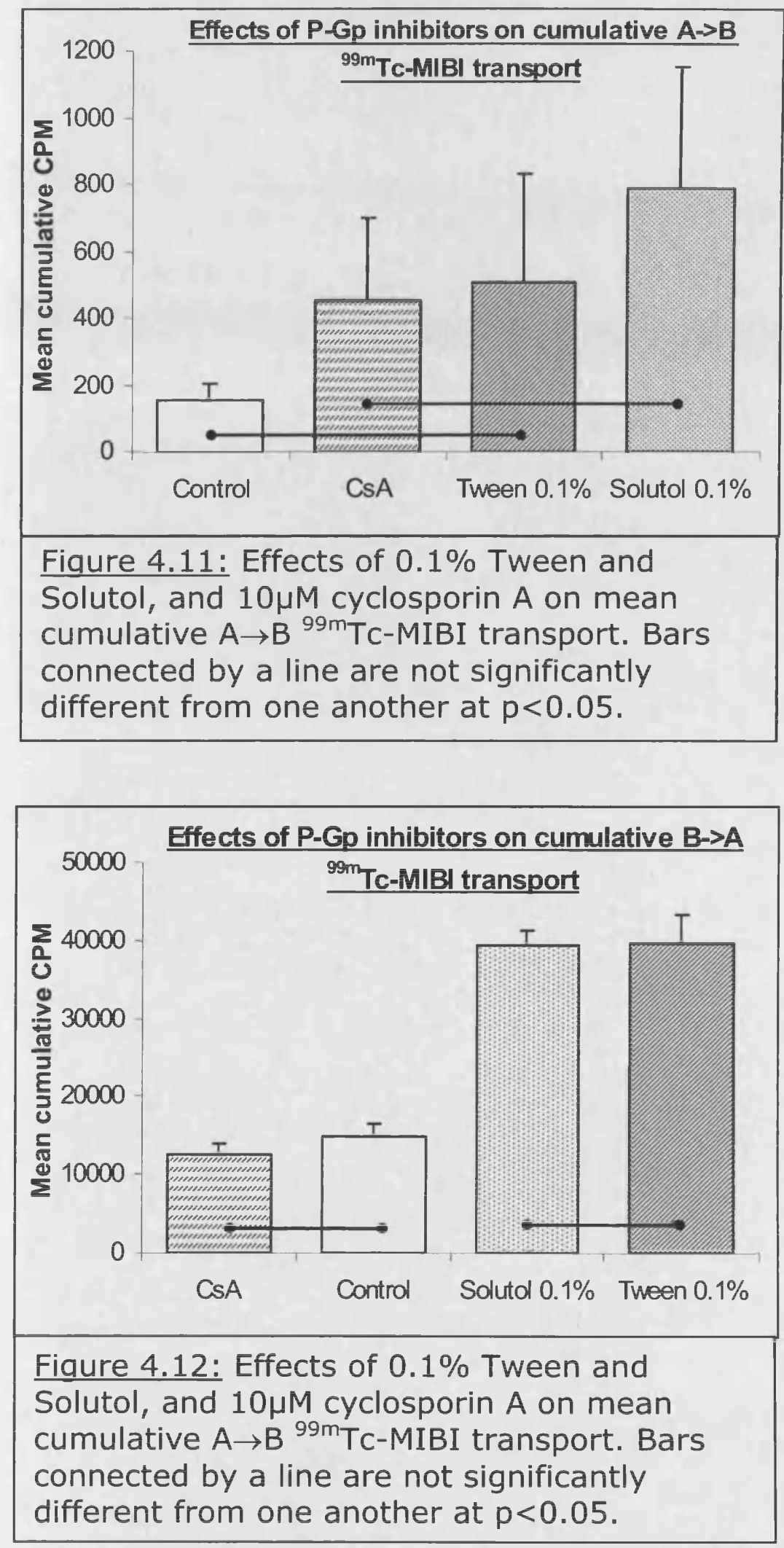


\subsubsection{Bi-directional ${ }^{99 \mathrm{~m}} \mathrm{Tc}$ transport}

Table 4.8 shows the permeability coefficients of free ${ }^{99 \mathrm{~m}} \mathrm{Tc}$ across MDCK-MDR1 monolayers. No lag time was evident.

Table 4.8: $A \rightarrow B$ and $B \rightarrow A$ permeability coefficients of ${ }^{99 m} \mathrm{Tc}$ across MDCK-MDR1 monolayers.

\begin{tabular}{|c|c|c|c|c|c|c|c|c|}
\hline & \multicolumn{8}{|c|}{${ }^{99 \mathrm{~m}}$ Tc permeability coefficient $\left(\mathrm{cm} \mathrm{sec}-1, \times 10^{-6}\right)$} \\
\hline & 1 & 2 & 3 & 4 & 5 & 6 & Mea & ( \pm SD) \\
\hline$A B$ & 1.55 & 1.34 & 1.07 & 1.02 & 0.99 & 1.45 & 1.24 & $( \pm 0.24)$ \\
\hline BA & 1.03 & 0.90 & 0.90 & 0.81 & 0.71 & & 0.87 & $( \pm 0.12)$ \\
\hline
\end{tabular}

\subsection{Discussion}

The most profound effects on $\mathrm{A} \rightarrow \mathrm{B}^{3} \mathrm{H}$-vinblastine transport across the MDCK-MDR1 monolayers were seen with the surfactants Tween 80 and Solutol HS15. These excipients both significantly increased the permeability coefficient of ${ }^{3} \mathrm{H}$-vinblastine across the cell monolayer, at concentrations of $1 \%$ and $0.1 \%$. As sucrose permeability was also increased at $1 \%$, the effects seen at this concentration may be due to a general increase in permeability of the cell monolayer. However, the effects seen on the transport of ${ }^{3} \mathrm{H}$-vinblastine and not ${ }^{14} \mathrm{C}$-sucrose at $0.1 \%$ suggest a $\mathrm{P}$-Gp specific effect of Solutol and Tween. 
However, $0.1 \%$ Tween 80 did not significantly increase $A \rightarrow B{ }^{3} \mathrm{H}-$ vinblastine permeability in the bi-directional transport study, despite doing so in the other two $A \rightarrow B$ studies. The excipient did increase the $A \rightarrow B{ }^{3} \mathrm{H}$-vinblastine permeability coefficient by 1.5 -fold in these studies; the fact that this was not statistically significant may be due to the high standard deviations in this study. The highly variable ${ }^{14} \mathrm{C}$-sucrose permeabilities in this batch of cells suggest that the monolayers had variable permeabilities, which may be the reason for the large standard deviations. The significant effects seen in the other two studies clearly demonstrate that Tween can inhibit P-Gp in MDCK-MDR1 cells at this concentration.

P-Gp inhibition by Solutol has also been reported by other authors. Solutol has been reported to reduce the IC50 of colchicine, vinblastine and doxorubicin, and increase accumulation of rhodamine 123, in KB85 and KB8511 cells, suggesting inhibition of P-Gp (Coon et al., 1991). Woodcock et al. (1992) reported that Solutol inhibited P-Gp (demonstrated by increased daunorubicin accumulation) in CEM R100 leukemia cells, at a concentration of $0.01 \%$. Cornaire et al. (2004) reported that Solutol increased digoxin absorption from the everted rat gut sac model, at concentrations of $0.05 \%$ and above. 
Solutol is often used in pre-clinical studies at doses of $300 \mathrm{mg} / \mathrm{kg}$ (10\% 3ml/kg; M. DeMatas, personal communication). This would result in theoretical plasma concentrations of up to $0.7 \%$ in the rat, not accounting for distribution. The present studies demonstrated a clear effect at $0.1 \%$; it is therefore theoretically possible that Solutol could affect drug pharmacokinetics through P-gp inhibition in vivo, at doses commonly used in pre-clinical studies. There are reports in the literature where Solutol has altered the pharmacokinetics of P-Gp substrates in vivo (discussed in Chapter 1), and this will also be investigated in Chapter 5.

Tween 80 has previously been reported to inhibit P-Gp in Caco-2 cells (as demonstrated by changes in ${ }^{3} \mathrm{H}$-taxol transport), at a concentration of $0.05 \%$ (Hugger et al., 2002). Tween increased the $A \rightarrow B$ permeability coefficient, reduced the $B \rightarrow A$ permeability coefficient, and reduced the $B A / A B$ ratio from 13.2 to 5.3. However, in the same study it did not inhibit P-Gp in MDCK-MDR1 cells at this concentration, having no effect on $A \rightarrow B$ or $B \rightarrow A$ permeability coefficients, or the $B A / A B$ ratio. It is of interest that no effect was seen in this cell line, as the present studies have shown an effect at $0.1 \%$ in the same cell line. This may be due to the fact that the concentration used was lower than in the present studies, although the magnitude of the effect seen at $0.1 \%$ would suggest that effects could be seen at lower concentrations. It is also of interest that 
Tween reduced $\mathrm{B} \rightarrow \mathrm{A}{ }^{3} \mathrm{H}$-taxol transport in the Caco- 2 monolayers in Hugger et al.'s study (2002), but did not affect the $B \rightarrow A$ transport of ${ }^{3} \mathrm{H}$-vinblastine in the present study. This may be due to differences between the cell lines used, or differences in the affinities of the substrates for efflux transporters; as discussed below, transporters other than P-Gp may be responsible for the lack of effect of Tween on $B \rightarrow A{ }^{3} \mathrm{H}$-vinblastine transport seen in the present study. Woodcock et al. (1992) reported that Tween inhibited P-Gp (demonstrated by increased daunorubicin accumulation) in CEM R100 leukemia cells, at a concentration of 0.1\%. Bogman et al. (2003) reported that Tween 80 inhibited P-Gp (as demonstrated by reduced rhodamine efflux) in P338 cells. Cornaire et al. $(2004,2000)$ reported that Tween 80 increased digoxin absorption in the everted rat gut sac model, although no effect was seen at concentrations below $0.5 \%$. Shono et al. (2004) reported that Tween 80 affected the transport of rhodamine-123 across rat jejunal membrane in vitro. The ratio of serosal-mucosal transport to mucosal-serosal transport was reduced, suggesting inhibition of P-Gp. Ellis et al. (1996) reported inhibition of etoposide elimination by Tween 80 in the isolated perfused rat liver, attributed to inhibition of P-Gp-mediated biliary excretion of the drug, or decreased/ delayed liver uptake due to drug entrapment in micelles. 
In pre-clinical studies, Tween 80 is commonly used at doses of $266 \mathrm{mg} / \mathrm{kg}$ ( $5 \% \mathrm{v} / \mathrm{v} 5 \mathrm{ml} / \mathrm{kg} ;$ M. DeMatas, personal communication). This would result in a theoretical plasma concentration of up to $0.4 \%$ (not accounting for distribution), higher than the concentration at which in vitro P-Gp inhibition was shown in the present studies. Although Tween is rapidly eliminated (van Tellingen et al., 1999), it is still theoretically possible that the surfactant could affect drug pharmacokinetics by P-Gp inhibition in vivo. Indeed, as described in Chapter 1, there are in vivo reports where Tween 80 appears to have inhibited P-Gp, and this will also be investigated in Chapter 5.

Cyclosporin A was used in the bi-directional transport studies for comparative purposes as a well-established P-Gp inhibitor. As expected, this inhibitor significantly increased ${ }^{3} \mathrm{H}$-vinblastine permeability in the $A \rightarrow B$ direction and reduced permeability in the $\mathrm{B} \rightarrow \mathrm{A}$ direction. Campbell et al. (2003), also using MDCK-MDR1 cells, reported that $10 \mu \mathrm{M}$ cyclosporin $\mathrm{A}$ increased ${ }^{3} \mathrm{H}$-vinblastine permeability in the $A \rightarrow B$ direction, and reduced ${ }^{3} \mathrm{H}$-vinblastine permeability in the $B \rightarrow A$ direction, such that the ratio of $B A / A B$ transport was reduced from 18.5 to approximately 1 . The effect shown in the present study was not as large as this; the $B A / A B$ ratio in the cyclosporin-treated monolayers was 5.9. This may be due to the slightly lower cyclosporin A concentration used. The BA/AB ratio 
in control monolayers in the present study is comparable with theirs (14.3 vs. 18.5 ), although the ${ }^{3} \mathrm{H}$-vinblastine permeability in each direction is lower than theirs $(0.46 \pm 0.12$ vs. approx. $1.1 \mathrm{~cm} / \mathrm{sec}$ $x 10^{-6} A \rightarrow B ; 6.60 \pm 0.46$ vs approx. $\left.20 \mathrm{~cm} / \mathrm{sec} \times 10^{-6} \mathrm{~B} \rightarrow A\right)$.

In contrast to cyclosporin A, neither Solutol HS15 or Tween 80 $(0.1 \%)$ significantly affected $B \rightarrow A{ }^{3} \mathrm{H}$-vinblastine permeability in the bi-directional studies. This is of interest, as inhibition of P-Gp would be expected to give a reduction in $B \rightarrow A$ transport, due to inhibition of P-Gp-mediated transport in this direction. The excipients were present in the media on both sides of the cell monolayer, so the lack of effect on $B \rightarrow A$ transport is not due to lack of access of the inhibitors to P-Gp on the apical membrane. A possible explanation is that the excipients also inhibited a transporter on the basolateral membrane of the cells. MRP1, 3 and 5 are expressed on the basolateral membrane of polarised kidney epithelia (Schinkel and Jonker, 2003); inhibition of one of these transporters may be a possible reason for the absence of the expected reduction in $B \rightarrow A$ transport.

Transporters other than P-Gp are also expressed on the apical membrane of MDCK-MDR1 cells. MRP2, MRP4 and BCRP are also expressed here (Schinkel and Jonker, 2003). As vinblastine is not a substrate for BCRP (Litman et al., 2000; Cisternino et al., 2004) or 
MRP4 (Adachi et al., 2002), these transporters are unlikely to have affected the results. However, vinblastine is a substrate for MRP2. It is possible that some of the effects seen on $A \rightarrow B$ transport could be due to inhibition of this transporter; however, as P-Gp is overexpressed in MDCK-MDR1 cells it is likely that the majority of the effects seen are due to interactions with P-Gp rather than MRP2.

PEG 400 and PG both increased ${ }^{3} \mathrm{H}$-vinblastine permeability across the cell monolayer at $1 \%$ without affecting ${ }^{14} \mathrm{C}$-sucrose permeability, suggesting an inhibitory effect on P-Gp. However, the effect was lost at $0.1 \%$, demonstrating that these excipients are less potent P-Gp inhibitors than Tween 80 and Solutol HS15. When the transport study with PG was repeated, there was no longer a significant effect on ${ }^{3} \mathrm{H}$-vinblastine transport at either concentration, although the mean permeability coefficient at $5 \%$ was significantly different from that at $1 \%$. As the effect was not reproducible, it is possible that the effect seen in the first experiment was merely an artefact. Indeed, in the initial $1 \%$ experiment PG increased ${ }^{14} \mathrm{C}$ sucrose permeability coefficient by 1.7 -fold (although this was not significantly different from the control), corresponding to the 1.6fold increase seen in ${ }^{3} \mathrm{H}$-vinblastine permeability coefficient. A slight increase in monolayer permeability may therefore be the reason for the significant effect seen on ${ }^{3} \mathrm{H}$-vinblastine permeability coefficient in the first experiment. In the experiment with $1 \%$ and $5 \%$ PG, $1 \%$ 
PG did not increase ${ }^{14} \mathrm{C}$-sucrose permeability coefficient at all, nor was any significant effect seen on ${ }^{3} \mathrm{H}$-vinblastine permeability coefficient. Although ${ }^{3} \mathrm{H}$-vinblastine permeability coefficients at the two PG concentrations were significantly different from each other, this may again be due to a slight increase in monolayer permeability, as 5\% PG increased ${ }^{3} \mathrm{H}$-vinblastine permeability coefficient by 1.3 -fold compared to control and ${ }^{14} \mathrm{C}$-sucrose permeability coefficient by 1.2 -fold. No effect of PG on P-Gp has been reported in the literature. Cornaire et al. (2004) reported that PG did not affect permeability of the P-gp substrate digoxin across an everted rat gut sac, at concentrations of $0.5 \%$ and below. In pre-clinical studies, doses of PG up to $1036 \mathrm{mg} / \mathrm{kg}$ are used $(50 \%$ $2 \mathrm{ml} / \mathrm{kg}$; Marcel DeMatas, personal communication), which could result in theoretical plasma concentrations of up to $0.16 \%$ in the rat, assuming distribution into total body water. It therefore seems unlikely that an inhibitory effect of PG on P-Gp would be seen in vivo at doses commonly used in pre-clinical studies, even if the significant effect seen at $1 \%$ in the first study is not due to a general increase in monolayer permeability. Further repeats of the experiment would enable confirmation of whether this excipient can affect P-Gp. However, as the effect, if any, is unlikely to be of relevance in vivo, this would be of limited value. 
Previously, PEG 400 has been reported to inhibit P-Gp in the excised rat jejunum (Johnson et al., 2002) at 1\%, as assessed by digoxin transport. However, it had no significant effect on digoxin transport in the everted rat gut sac model, at concentrations of $0.5 \%$ and below (Cornaire et al., 2004). PEG 300 was reported to inhibit P-Gp in Caco-2 and MDCK-MDR1 cells at a concentration of $20 \%$ (Hugger et al., 2002); however, this is not a realistic concentration to extrapolate to the in vivo situation. In pre-clinical studies, PEG 400 is used at doses of up to $676 \mathrm{mg} / \mathrm{kg}(60 \% 1 \mathrm{ml} / \mathrm{kg} ; \mathrm{M}$. DeMatas, personal communication), resulting in a theoretical blood concentration of $0.9 \%$, not accounting for distribution. This means that it is unlikely that PEG 400 would affect drug pharmacokinetics through $\mathrm{P}-\mathrm{Gp}$ inhibition in vivo, as no effects were seen at $0.1 \%$, and the effect seen at $1 \%$ is small, and may not be reproducible. The literature does not contain any reports of PEG 400 affecting the disposition of P-Gp substrates.

DMSO did not show any inhibitory effects on P-Gp at either $1 \%$ or $0.1 \%$, nor were any effects on membrane permeability $\left({ }^{14} \mathrm{C}\right.$-sucrose permeability) demonstrated for this excipient. There are no reports in the literature which suggest an effect of DMSO on P-Gp either in vitro or in vivo. 
As discussed in Chapter 2, it has been reported that the erythromycin breath test (EMBT) can be affected by P-Gp as well as alterations in metabolic capacity (Kurnik et al., 2006). The P-Gp inhibition data obtained in this chapter are therefore useful in clarifying whether effects on P-Gp were a factor in the results obtained. The only excipient to significantly affect the EMBT was DMSO, which had inhibitory effects at a dose of $3520 \mathrm{mg} / \mathrm{kg}$. As inhibition of P-Gp in the EMBT resembles enzyme induction rather than inhibition, it did not appear that inhibition of P-Gp was a factor in the result seen. The lack of significant effect of DMSO on P-Gp in the present studies appears to confirm this.

In the bi-directional transport studies, ${ }^{99 \mathrm{~m}} \mathrm{TC}$-MIBI had a $\mathrm{BA} / \mathrm{AB}$ ratio of 69.0 , compared to 14.3 for vinblastine. This confirms that ${ }^{99 \mathrm{~m}} \mathrm{Tc}-$ MIBI is a good substrate for transporters, and that they were important determinants of its passage across the cell monolayers. The significant increases in $A \rightarrow B$ permeability coefficient and cumulative $A \rightarrow B{ }^{99 m}$ Tc-MIBI transport over 80 minutes caused by Solutol HS15 in the bi-directional ${ }^{99 m}$ Tc-MIBI studies suggest that the excipient increased ${ }^{99 \mathrm{~m}} \mathrm{Tc}$-MIBI transport across the monolayer by P-Gp inhibition. This is in line with the effects of the Solutol on $A \rightarrow B{ }^{3} \mathrm{H}$-vinblastine transport described above. Variability in the monolayers appears to have prevented the effects of Tween 80 on $A \rightarrow B$ transport from achieving significance, as seen in the bi- 
directional ${ }^{3} \mathrm{H}$-vinblastine studies described above. However, the 4.4-fold increase in average $A \rightarrow B$ permeability coefficient suggests that Tween did have an effect on ${ }^{99 m}$ Tc-MIBI transport in this direction, consistent with inhibition of P-Gp. Similarly, the 4-fold increase in ${ }^{99 m}$ Tc-MIBI $A \rightarrow B$ permeability coefficient caused by the P-Gp inhibitor Cyclosporin A on A $\rightarrow B$ MIBI transport was not significant, apparently due to large standard deviations caused by variability in the cell monolayers.

The increases in $B \rightarrow A$ permeability and cumulative $B \rightarrow A$ transport of ${ }^{99 m}$ Tc-MIBI caused by Solutol and Tween 80 are of interest, as if the excipient were inhibiting P-Gp alone we would expect to see a reduction in $B \rightarrow A$ transport, due to inhibition of $P-G p$ which pumps its substrates in this direction. The increase in $B \rightarrow A{ }^{99 m}$ Tc-MIBI transport seen suggests inhibition of a transporter on the basolateral side of the membrane. MRP1, 3 and 5 are known to be expressed on the basolateral side of polarised kidney epithelia (Schinkel and Jonker, 2003). ${ }^{99 \mathrm{~m}} \mathrm{Tc}$-MIBI has been reported to be a substrate for MRPs 1 and 2 (Hendrikse et al., 1998; Utsunomiya et al., 2000; Hendrikse et al., 2004); it is possible that inhibition of MRP1 could be responsible for the increased $B \rightarrow A{ }^{99 m}$ Tc-MIBI transport. However, neither excipient increased the $B \rightarrow A$ transport of ${ }^{3} \mathrm{H}$-vinblastine, which is also a substrate for MRP1 (Schinkel and Jonker, 2003). The effect therefore may not be due inhibition of 
MRP1, unless this protein has a greater effect on the disposition of ${ }^{99 m}$ Tc-MIBI than on ${ }^{3} \mathrm{H}$-vinblastine. This increase in $\mathrm{B} \rightarrow \mathrm{A}{ }^{99 \mathrm{~m}}$ Tc-MIBI transport also meant that no reduction in $B A / A B$ ratio was seen for Tween 80 , despite the increase in $A \rightarrow B$ transport caused by this excipient, and only a small reduction in $B A / A B$ ratio was seen for Solutol.

The lack of effect of the P-Gp inhibitor Cyclosporin A on ${ }^{99 m}$ Tc-MIBI $B \rightarrow A$ transport is of interest, as if the disposition of ${ }^{99 m}$ Tc-MIBI was primarily due to $\mathrm{P}-\mathrm{Gp}$, a reduction in $\mathrm{B} \rightarrow \mathrm{A}$ transport would be expected due to loss of P-Gp mediated efflux in this direction. This suggests that other transporters were also important in determining the disposition of ${ }^{99 m}$ Tc-MIBI in this experiment. Cyclosporin A is also known to be an inhibitor of MRP, and has been shown to increase ${ }^{99 m}$ Tc-MIBI uptake in cells expressing MRP but not P-Gp (Utsunomiya et al., 2000). It is possible that inhibition of efflux by MRP on the basolateral membrane is causing an increase in $B \rightarrow A$ transport, masking any reduction in $B \rightarrow A$ transport that would be seen due to inhibition of P-Gp and MRP on the apical membrane.

The permeability coefficients of free ${ }^{99 \mathrm{~m}} \mathrm{Tc}$ across the monolayers in both directions were clearly very different to those for ${ }^{99 m}$ Tc-MIBI in all of the treatment groups. This confirms that transport of ${ }^{99 \mathrm{~m}} \mathrm{Tc}-$ MIBI and not free ${ }^{99 m}$ Tc was measured, i.e. that the ${ }^{99 m}$ Tc-MIBI 
complex had not broken down. The $A \rightarrow B$ permeability coefficient of the free ${ }^{99 \mathrm{~m}} \mathrm{Tc}$ is larger than that in the $\mathrm{B} \rightarrow \mathrm{A}$ direction. This trend is also apparent in the cumulative counts transported over $\mathbf{8 0}$ minutes, suggesting that the transport of free ${ }^{99 m}$ Tc may not be due to simple diffusion alone in MDCK-MDR1 cells.

\subsubsection{Conclusions}

The present studies demonstrate that Tween 80 and Solutol HS15 can inhibit P-Gp in vitro at a concentration of $0.1 \%$, as assessed by $A \rightarrow B{ }^{3} \mathrm{H}$-vinblastine transport across MDCK-MDR1 monolayers. The fact that Solutol HS15 significantly increased the $A \rightarrow B$ transport of ${ }^{99 \mathrm{~m}} \mathrm{Tc}-$ MIBI suggests that this excipient may be able to affect the in vivo disposition of ${ }^{99 m}$ Tc-MIBI, by increasing its $A \rightarrow B$ transport across biological barriers where $\mathrm{P}-\mathrm{Gp}$ restricts movement in this direction. Solutol HS15 therefore merits further investigation in in vivo studies with ${ }^{99 \mathrm{~m}} \mathrm{Tc}$-MIBI. Although Tween 80 did not significantly affect $A \rightarrow B{ }^{99 m}$ Tc-MIBI transport, the fact that it increased ${ }^{99 \mathrm{~m}}$ Tc-MIBI permeability coefficient by 4.4 -fold, and significant $P-G p$ inhibition was seen in the $A \rightarrow B{ }^{3} \mathrm{H}$-vinblastine studies, suggest that this excipient is also worth further investigation in vivo. 
However, the fact that Tween and Solutol significantly increased the $B \rightarrow A$ transport of ${ }^{99 m}$ TC-MIBI suggests that the excipients can inhibit transporters other than P-Gp for which ${ }^{99 \mathrm{~m}}$ TC-MIBI is a substrate. This may complicate the interpretation of data from the in vivo ${ }^{99 m}$ Tc-MIBI model.

\subsubsection{Further work}

Having identified suitable excipients, their effects on the disposition of ${ }^{99 \mathrm{~m}}$ Tc-MIBI in vivo can be systematically investigated.

It would be useful to repeat the bi-directional transport studies, as the monolayers used in this experiment showed variable (although not significantly different) ${ }^{14} \mathrm{C}$-sucrose permeabilities in some of the groups. However, cost and time limitations prevent this. Indeed, the required information has been obtained from these experiments, in that suitable excipients for use in in vivo ${ }^{99 m}$ Tc-MIBI studies have been identified.

It may also be of interest to analyse the ${ }^{99 m}$ Tc-MIBI transport data using a model which can take the lag time into account, rather than modelling from the point where transport was apparent after the lag time had elapsed. However, as the cumulative transport and permeability coefficient data were in agreement regarding which 
treatments had significant effects, this would add little to the interpretation of the data.

It may also be of interest to obtain cell lines overexpressing MRP1/2 to investigate the inhibitory effects of the selected excipients on these proteins, and how this affects ${ }^{99 \mathrm{~m}}$ Tc-MIBI transport. 


\section{Chapter 5:}

\section{In vivo disposition of ${ }^{99 \mathrm{~m}}$ Tc-MIBI and ${ }^{99 m}$ Tc-HIDA}




\section{Contents}

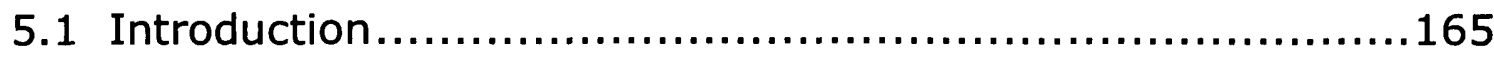

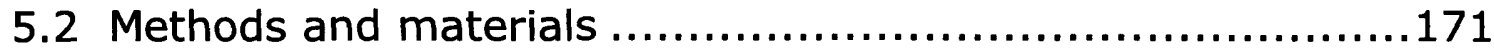

5.2.1 Materials..................................................... 171

5.2.2 Methods.................................................. 172

5.3 Results............................................................. 177

5.3.1 ${ }^{99 \mathrm{~m}}$ TC-MIBI ................................................

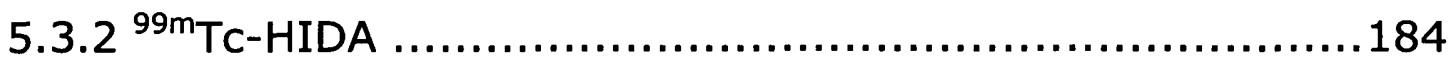

5.3.3 Effect of injection site retention............................190

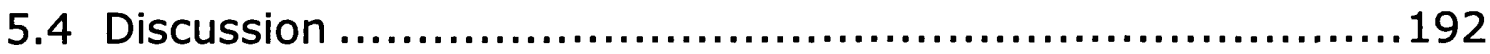

5.4.1 Conclusions .............................................200

5.4 .2 Further work ...........................................201 


\subsection{Introduction}

The studies described in the previous chapter demonstrated that various excipients can inhibit P-Gp in vitro, as shown by their effects on the transport of ${ }^{3} \mathrm{H}$-vinblastine and ${ }^{99 \mathrm{~m}} \mathrm{TC}-\mathrm{MIBI}$ across MDCKMDR1 cells. It is therefore now pertinent to investigate whether these effects occur in vivo, to an extent which could significantly impact upon drug pharmacokinetics.

A recent publication by Hendrikse et al. (2004) reported the suitability of ${ }^{99 \mathrm{~m}} \mathrm{Tc}$ MIBI and another compound, ${ }^{99 \mathrm{~m}} \mathrm{Tc}-\mathrm{HIDA}$, as probes for biliary excretion due to P-gp and MRP2. The authors have previously demonstrated that, while ${ }^{99 \mathrm{~m}} \mathrm{Tc}$-MIBI is a substrate for $\mathrm{P}$ Gp and MRP1 in vitro (Hendrikse et al. 1998), ${ }^{99 \mathrm{~m}} \mathrm{Tc}-\mathrm{HIDA}$ is a substrate for MRP1 but not P-Gp. This lead them to hypothesise that use of the two compounds could provide information about P-Gp and MRP in biliary elimination. The biliary elimination of the two radiopharmaceuticals was then investigated in vivo. The elimination of ${ }^{99 \mathrm{~m}} \mathrm{Tc}-\mathrm{HIDA}$ into bile was impaired in MRP2 deficient rats, compared to control rats. The amount of radioactivity eliminated into the bile over 20 minutes in the MRP2 deficient rats was only $44 \%$ of that in controls. Additionally, glutathione depletion (in order to reduce MRP transport, as many MRP substrates are transported as glutathione conjugates) reduced the biliary elimination of ${ }^{99 m} \mathrm{Tc}-$ 
HIDA in control rats, to give a similar elimination profile to that seen in MRP2 deficient rats. This demonstrates that MRP2 is important in the biliary elimination of ${ }^{99 m}$ Tc-HIDA in vivo. Although MRP1 was investigated in the in vitro studies, whereas MRP2 is of interest in vivo, the authors state that this was justified due to the overlapping substrate specificities of these transporters. There were no differences in the biliary elimination of ${ }^{99 \mathrm{~m}} \mathrm{Tc}$-MIBI between control and MRP2 deficient rats. This lack of difference was attributed to upregulation of other transporters in the MRP2 deficient rats. Glutathione depletion reduced biliary elimination of ${ }^{99 \mathrm{~m}}$ Tc-MIBI in control rats, demonstrating that MRP2 is involved in the biliary elimination of ${ }^{99 \mathrm{~m}}$ Tc-MIBI in vivo.

Similar information was obtained from in vivo gamma scintigraphy studies (Hendrikse et al., 2004). Sequential images were taken over a period of one hour. Elimination of ${ }^{99 \mathrm{~m}} \mathrm{Tc}$-HIDA from the liver was impaired in MRP2 deficient rats compared to control rats, with $t_{1 / 2 \text { (liver) }}$ increasing from 7 minutes to 40 minutes. There were no differences in the liver elimination of ${ }^{99 \mathrm{~m}} \mathrm{Tc}$-MIBI between control and MRP2 deficient rats.

There were no significant differences in the plasma clearance of either compound, demonstrating that, although ${ }^{99 m}$ Tc-HIDA elimination from the liver was impaired in MRP2 deficient rats, its 
uptake into the liver was not affected. The authors concluded that, due to their differential substrate specificities, use of both ${ }^{99 \mathrm{~m}} \mathrm{Tc}$ MIBI and ${ }^{99 m}$ Tc-HIDA could provide information about biliary elimination due to $\mathrm{P}-\mathrm{Gp}$ and MRP2 in vivo. The importance of MRP2 in ${ }^{99 m}$ Tc-HIDA biliary elimination is also evidenced by the fact that it's biliary elimination is impaired in patients with Dubin-Johnson syndrome (Bar-Meir et al. 1982), a condition where MRP2 is defective. ${ }^{99 m}$ Tc-MIBI and ${ }^{99 m}$ Tc-HIDA therefore appear useful for investigating the effects of excipients on these efflux transporters in vivo, and the impact any such effects could have on the disposition of compounds which are substrates for these transporters.

It would not be necessary to use all of the components of Hendrikse et al.'s method to investigate the effects of excipients on biliary elimination. The biliary elimination data described above were obtained by bile duct cannulation. This is an invasive surgical procedure, requiring deep anaesthesia which has the potential to affect physiological processes and hence experimental outcomes. The gamma scintigraphy studies also enabled impaired elimination of ${ }^{99 \mathrm{~m}} \mathrm{Tc}$-HIDA to be detected in MRP2 deficient rats; this technique has the advantages of being non-invasive, and requiring minimal anaesthesia. 
The organs of interest in the elimination of ${ }^{99 \mathrm{~m}} \mathrm{Tc}$ MIBI and ${ }^{99 \mathrm{~m}} \mathrm{Tc}-$ HIDA (liver, kidney, small intestine) are overlaid on scintigraphic images, due to their positions in the body of the rat. Although this did not prevent the authors of the above study from detecting delayed biliary elimination of ${ }^{99 \mathrm{~m}} \mathrm{Tc}-\mathrm{HIDA}$ in MRP2 deficient rats, it is likely to make detection of more subtle changes, resulting from a partial inhibition of the transport proteins, difficult to discern. Dissection of the rats after the in vivo imaging period, and subsequent counting of the radioactivity in individual organs, is a potential solution to this problem. Although this will only give information about ${ }^{99 \mathrm{~m}} \mathrm{Tc}$ MIBI and ${ }^{99 \mathrm{~m}} \mathrm{Tc}$-HIDA distribution at a single timepoint, the combination of dissection with the in vivo scintigraphy will maximise the information that can be gained from the experiments, and should increase their ability to detect any effects of the excipients.

Other authors have successfully used dissection techniques to demonstrate the effects of P-Gp on ${ }^{99 m}$ Tc-MIBI distribution. Barbarics et al. (1998) investigated the effects of the P-Gp inhibitor SDZ PSC 833 on ${ }^{99 m}$ TC-MIBI biodistribution in mice. 15 minutes after ${ }^{99 \mathrm{~m}}$ Tc-MIBI administration, mice treated with the P-Gp inhibitor showed increased ${ }^{99 m}$ Tc-MIBI levels in resistant tumour tissue, lung and spleen, with smaller increases in brain, blood and muscle uptake (no statistical analysis of non-tumour tissue levels was 
reported). The 15 minute study duration was selected to show adequate differences in resistant and non-resistant tumour uptake; the authors comment that greater differences in other tissues may have been observed at later timepoints. Dyzlewski et al. (2002) investigated differences in ${ }^{99 m}$ Tc-MIBI blood, brain and liver levels in wild-type and mdr1a/1b (-/-) P-Gp knockout mice. Dissection was performed at 5 or 120 minutes after dosing. Delayed liver clearance of ${ }^{99 \mathrm{~m}} \mathrm{Tc}-\mathrm{MIBI}$ in the knockout mice compared to wild-type was evident, demonstrating that $\mathrm{P}-\mathrm{Gp}$ is also important in the elimination of ${ }^{99 \mathrm{~m}}$ Tc-MIBI from the liver. Brain levels of ${ }^{99 \mathrm{~m}}$ Tc-MIBI were increased in the knockout mice at both timepoints, but blood levels were similar.

Hendrikse et al. (2004) reported that there were no discernible differences in the levels of ${ }^{99 m}$ Tc-MIBI or ${ }^{99 m}$ Tc-HIDA in various organs (not specified) between control and MRP2 deficient rats, an hour after dosing. However, examination of their graphs depicting differences in liver and biliary elimination of ${ }^{99 m}$ Tc-HIDA reveals that most of the difference between groups has dissipated by this time. It is possible that dissection at an earlier timepoint would have enabled differences to be observed. Also, as no differences were observed in biliary elimination or $\mathrm{t}_{1 / 2 \text { (liver) }}$ of ${ }^{99 \mathrm{~m}}$ Tc-MIBI between control and MRP2 deficient rats, it would not be expected that changes would be apparent upon dissection. 
The combination of scintigraphy using ${ }^{99 \mathrm{~m}}$ Tc-MIBI and ${ }^{99 \mathrm{~m}}$ Tc-HIDA, and subsequent dissection, therefore appears to be a useful method to investigate the effects of excipients on P-Gp and MRP mediated distribution and elimination in vivo. It will be of particular interest to determine the effects of Solutol and Tween 80 on biliary elimination, as studies in the literature seem to suggest that these excipients may inhibit the in vivo biliary elimination of drugs which are P-Gp substrates (Bittner et al., 2003b; Zhang et al., 2003), but methodological limitations did not enable this to be confirmed. Solutol HS15 and Tween 80 also showed significant effects on P-Gp in the cell studies described in the previous chapter, and so were selected for investigation in these experiments (time and resource limitations prevent the testing of large numbers of excipients in this model). This series of experiments therefore aims to investigate the effects of Solutol HS15 and Tween 80 on the in vivo disposition of ${ }^{99 m}$ TC-MIBI and ${ }^{99 m}$ Tc-HIDA. 


\subsection{Methods and materials}

\subsubsection{Materials}

Tween 80, aluminium oxide TLC plates, silica TLC plates, and ethanol were obtained from Fisher, UK. N-(2,6dimethylphenylcarbamoylmethyl) iminodiacetic acid (HIDA), methylethylketone, and stannous chloride were obtained from Sigma-Aldrich, UK. Solutol HS15 was a kind gift from AstraZeneca, Alderley Park, UK. The kit for preparation of ${ }^{99 \mathrm{~m}}$ Tc-MIBI (Cardiolite ${ }^{\circledR}$, Bristol-Myers-Squibb) and ${ }^{99 \mathrm{~m}} \mathrm{Tc}$ were obtained from Medical Physics, University Hospital Wales.

\section{Animals}

Male Sprague-Dawley rats (230-290g) (Harlan) were group housed on a 12 hour light/dark cycle, with access to water and standard laboratory rodent diet ad libitum. 


\subsubsection{Methods}

\subsubsection{Preparation of ${ }^{99 \mathrm{~m}}$ Tc-MIBI and ${ }^{99 \mathrm{~m}}$ Tc-HIDA}

${ }^{99 m}$ Tc-MIBI was prepared from a commercially available kit

(Cardiolite ${ }^{\circledR}$ ). The contents of each vial were divided into 4-5

aliquots and stored under nitrogen until use. Labelling with ${ }^{99 \mathrm{~m}} \mathrm{Tc}$ was carried out according to the manufacturers instructions. When an aliquot which had been stored under nitrogen was used, $0.13 \mathrm{mg}$ of stannous chloride were added to the reaction mixture to ensure adequate labelling. Radiochemical purity, determined by TLC on aluminium oxide plates as described by the manufacturer, was 95$98 \%$.

${ }^{99 m}$ Tc-HIDA was prepared using the method of Hendrikse et al. (2004). $10 \mathrm{mg}$ of HIDA was dissolved in $0.15 \mathrm{ml}$ of $0.5 \mathrm{M} \mathrm{NaOH}$. $0.09 \mathrm{ml}$ of $0.1 \mathrm{M} \mathrm{HCl}$ was added to neutralise the solution, and saline was added to increase the total volume for stirring to $1 \mathrm{ml} .0 .13 \mathrm{ml}$ of a freshly prepared solution of stannous chloride $(1 \mathrm{mg} / \mathrm{ml}$ in freshly boiled and cooled water) and $30-50 \mathrm{MBq}$ of ${ }^{99 \mathrm{~m}} \mathrm{Tc}$ as pertechnetate were added, and the solution was stirred for 20 minutes. Further saline was added if necessary to provide a suitable 
volume for dosing. Radiochemical purity, determined by TLC (silica gel plates, methylethylketone mobile phase), was $91-93 \%$

\subsubsection{Disposition studies in rats}

\section{Anaesthesia}

Rats were sedated with $0.3-0.5 \mathrm{ml}$ Hypnorm (fentanyl citrate $0.315 \mathrm{mg} / \mathrm{ml}$ and fluanisone $10 \mathrm{mg} / \mathrm{ml}$, VetaPharma) intraperitoneally. Further doses of Hypnorm were administered as necessary during the imaging period. Intravenous injections of excipients, ${ }^{99 \mathrm{~m}} \mathrm{Tc}-\mathrm{MIBI}$ and ${ }^{99 \mathrm{~m}} \mathrm{Tc}-$ HIDA were carried out under isoflurane. Isoflurane was also used to immobilise the animals and assist with imaging where necessary.

\section{Dosing}

Each treatment group consisted of four rats. Rats were dosed with either ${ }^{99 \mathrm{~m}}$ Tc-MIBI or ${ }^{99 \mathrm{~m}}$ Tc-HIDA by i.v. injection. Rats in the excipient treatment groups received $160 \mathrm{mg} / \mathrm{kg}$ Solutol HS15 i.v. ( $20 \%$ solution, $0.8 \mathrm{ml} / \mathrm{kg}$ ), or $85 \mathrm{mg} / \mathrm{kg}$ Tween 80 i.v. ( $10 \%$ solution,

$0.8 \mathrm{ml} / \mathrm{kg}$ ) $1.5-3$ minutes prior to injection of ${ }^{99 \mathrm{~m}} \mathrm{Tc}-$ MIBI or ${ }^{99 \mathrm{~m}} \mathrm{Tc}-$ HIDA. Doses were administered into the penile vein. 


\section{Imaging}

Scintigraphic images were taken using a General Electric Maxicamera (model 400A). Magnification $\times 1.6$ was used for images of rats, blood and carcasses.

Immediately after injection of ${ }^{99 \mathrm{~m}}$ Tc-MIBI or ${ }^{99 \mathrm{~m}} \mathrm{Tc}-\mathrm{HIDA}$, rats were transferred to the gamma camera and an anterior image taken. Subsequent images were taken at 10, 20, 30, 45, and 60 minutes for ${ }^{99 \mathrm{~m}} \mathrm{Tc}-$ MIBI, and at 5, 10, 20, and 30 minutes for ${ }^{99 \mathrm{~m}}$ Tc-HIDA. Immediately after final image, a blood sample was taken, then rats were sacrificed and organs removed. Blood, organs and carcass were imaged on the gamma camera.

\subsubsection{Data processing}

\section{Calculation of dose}

The dose of ${ }^{99 m}$ Tc-MIBI or ${ }^{99 m}$ Tc -HIDA administered to each rat was calculated by the following method. A ROI was drawn around the rat on the first image taken after injection of the dose. The number of counts in this ROI were corrected for background, and the image duration. Injection site retention was accounted for by subtracting 
any dose retained at the injection site in the final image (corrected for background and time) from the whole body counts.

\section{Data processing for organs, carcass and blood}

The number of counts in organs, carcass and blood were obtained by drawing a ROI around the area of interest. The number of counts in the ROI were corrected for background and image duration. Images of syringes containing blood were also corrected for the volume of blood measured in the syringe. As the images of organs, blood and carcass were not taken at the same time after death for each rat, the counts were also corrected for time. The counts were then converted to a percentage of the dose given to each rat, obtained as described above. Correction of organ counts for blood background was not deemed to be necessary due to the very low activity in blood.

Some organs did not contain sufficient activity to be visible on the camera images. In order to estimate the maximum possible level of activity that these organs could contain, the minimum pixel size detected for any organ was multiplied by $1.5 x$ background, and converted to a percentage of dose. Levels in these organs are therefore reported as $<0.01 \%$ in the results section, as this corresponds to the maximum possible activity they could contain 
and not be visible on the camera images. No statistical analysis was performed using these values.

\section{Statistics}

Statistical tests were performed using SPSS 11 . Significance was assessed using a one-way ANOVA with Bonferroni's post-hoc test. Homogeneity of variance was assessed using Levene's test. Where variances were heterogeneous, significance was assessed using the Games-Howell test (Sokal and Rohlf, 1995); instances where this has been undertaken are highlighted in the results section. Differences where $p<0.05$ were considered significant. 


\subsection{Results}

\subsection{1 ${ }^{99 \mathrm{~m}}$ TC-MIBI}

Figure 5.1 shows the time course of ${ }^{99 \mathrm{~m}} \mathrm{Tc}$-MIBI disposition for a typical control rat. No differences between groups could be determined from the in vivo scintigraphic images. ${ }^{99 \mathrm{~m}} \mathrm{Tc}$-MIBI levels in each organ, expressed as \% of dose, for control, Solutol and Tween groups are shown in Tables 5.1, 5.2 and 5.3, respectively. All organs were visible on the post-dissection images. Some urine samples did not contain sufficient counts to be quantifiable. Similarly, the activity in blood was often so low that it was difficult to discern the region of interest from the background; therefore, no statistical analyses were performed on these data.

There was a deviation from the planned method for Rat $A$ in the Solutol group. Due to an error in making up the excipient solution, this rat received $0.8 \mathrm{ml} / \mathrm{kg}$ of a $40 \%$ Solutol solution rather than a $20 \%$ solution. However, most of this solution was retained at the injection site. Although it is not possible to ascertain the exact Solutol dose this rat received, it is likely that it did not receive a greater systemic dose than the other rats in this group, due to the volume retained at the site of injection. There are no obvious 
differences in the results obtained from this rat compared with the others in it's group. 


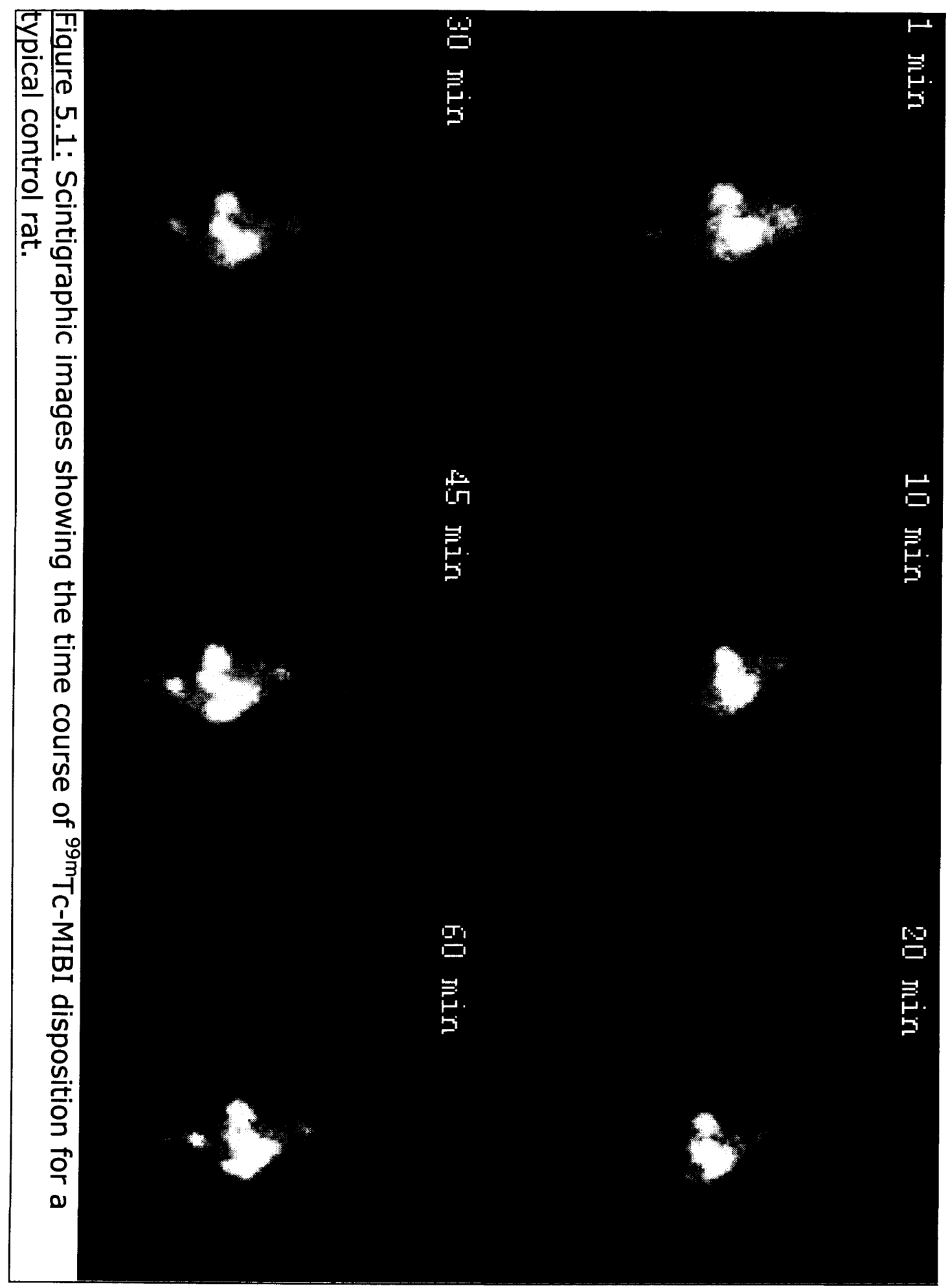


Table 5.1: Organ levels of ${ }^{99 \mathrm{~m}}$ Tc-MIBI, expressed at $\%$ of dose, in control rats.

\begin{tabular}{|c|c|c|c|c|cc|}
\cline { 2 - 8 } \multicolumn{1}{c|}{} & Rat A & Rat B & Rat C & Rat D & \multicolumn{2}{c|}{ Mean ( \pm SD) } \\
\hline Blood (per ml) & 0.03 & 0.03 & 0.04 & 0.03 & - & \\
\hline Carcass & 56.69 & 54.86 & 55.12 & 62.75 & 57.36 & $( \pm 3.69)$ \\
\hline Liver & 6.38 & 7.82 & 7.56 & 4.80 & 6.64 & $( \pm 1.38)$ \\
\hline Kidneys & 11.03 & 9.94 & 11.75 & 9.46 & 10.54 & $( \pm 1.04)$ \\
\hline Heart & 2.37 & 1.96 & 2.12 & 2.09 & 2.13 & $( \pm 0.17)$ \\
\hline Lungs & 0.76 & 0.90 & 0.90 & 0.85 & 0.85 & $( \pm 0.06)$ \\
\hline Stomach & 1.36 & 1.03 & 1.78 & 1.22 & 1.35 & $( \pm 0.32)$ \\
\hline Spleen & 0.83 & 0.83 & 1.27 & 0.87 & 0.95 & $( \pm 0.21)$ \\
\hline Urine & 1.65 & 1.86 & 0.05 & $*$ & - & \\
\hline Small intestine & 21.93 & 21.90 & 23.84 & 19.43 & 21.78 & $( \pm 1.81)$ \\
\hline Large intestine & 4.05 & 2.99 & 4.56 & 2.97 & 3.64 & $( \pm 0.79)$ \\
\hline Total & 107.06 & 104.08 & 108.96 & 104.44 & 106.13 & $( \pm 2.30)$ \\
\hline
\end{tabular}

*this rat's bladder was empty at the time of dissection, so no urine could be collected.

Table 5.2: Organ levels of ${ }^{99 \mathrm{~m}} \mathrm{Tc}-\mathrm{MIBI}$, expressed at $\%$ of dose, in rats pre-treated with Solutol HS15.

\begin{tabular}{|l|c|c|c|c|cc|}
\cline { 2 - 7 } \multicolumn{1}{c|}{} & Rat A & Rat B & Rat C & Rat D & \multicolumn{2}{c|}{ Mean ( \pm SD) } \\
\hline Blood (per ml) & $*$ & 0.04 & $*$ & $*$ & - & \\
\hline Liver & 65.80 & 57.43 & 70.85 & 59.47 & 63.39 & $( \pm 6.12)$ \\
\hline Kidneys & 7.18 & 7.15 & 4.30 & 8.57 & 6.80 & $( \pm 1.79)$ \\
\hline Heart & 11.54 & 8.47 & 8.88 & 10.50 & 9.85 & $( \pm 1.43)$ \\
\hline Lungs & 2.06 & 2.52 & 1.96 & 2.66 & 2.30 & $( \pm 0.34)$ \\
\hline Stomach & 0.75 & 1.27 & 0.88 & 1.00 & 0.98 & $( \pm 0.22)$ \\
\hline Spleen & 0.71 & 1.71 & 0.97 & 2.48 & 1.47 & $( \pm 0.80)$ \\
\hline Urine & 1.11 & 0.64 & 0.66 & 1.50 & 0.98 & $( \pm 0.41)$ \\
\hline Small intestine & 0.56 & 0.18 & $<0.01$ & 0.55 & - & \\
\hline Large intestine & 14.30 & 18.33 & 12.54 & 13.15 & 14.58 & $( \pm 2.60)$ \\
\hline Total & 2.45 & 2.91 & 1.89 & 2.26 & 2.38 & $( \pm 0.42)$ \\
\hline
\end{tabular}

*in these rats, a region of interest for the blood could not be discerned from background at all. 
Table 5.3: Organ levels of ${ }^{99 \mathrm{~m}}$ Tc-MIBI, expressed at $\%$ of dose, in rats pre-treated with Tween 80.

\begin{tabular}{|c|c|c|c|c|c|}
\hline & Rat A & Rat B & Rat C & Rat $D$ & Mean ( \pm SD) \\
\hline Blood (per ml) & 0.01 & 0.06 & 0.05 & 0.06 & - \\
\hline Carcass & 62.77 & 69.10 & 57.19 & 61.66 & $( \pm 4.91)$ \\
\hline \begin{tabular}{|l} 
Liver \\
\end{tabular} & 6.89 & 7.40 & 7.05 & 7.80 & $( \pm 0.41)$ \\
\hline Kidneys & 3.61 & 9.15 & 9.33 & 11.82 & $( \pm 3.46)$ \\
\hline \begin{tabular}{|l} 
Heart \\
\end{tabular} & 2.45 & 1.74 & 2.69 & 2.67 & $( \pm 0.44)$ \\
\hline Lungs & 0.66 & 0.75 & 0.93 & 0.60 & $( \pm 0.15)$ \\
\hline Stomach & 1.55 & 1.17 & 1.59 & 1.28 & $( \pm 0.20)$ \\
\hline Spleen & 0.71 & 0.72 & 0.37 & 0.94 & $( \pm 0.24)$ \\
\hline Urine & 4.44 & 0.07 & 0.62 & 0.35 & $( \pm 2.06)$ \\
\hline Small intestine & 16.38 & 14.34 & 17.58 & 16.03 & $16.08 \quad( \pm 1.34)$ \\
\hline Large intestine & 2.93 & 2.01 & 2.42 & 2.25 & $2.40 \quad( \pm 0.39)$ \\
\hline Total & 102.37 & 106.45 & 99.76 & 105.39 & $103.49( \pm 3.03)$ \\
\hline
\end{tabular}

A comparison of mean organ levels of ${ }^{99 \mathrm{~m}} \mathrm{Tc}$-MIBI in each group is shown in Table 5.4 .

Table 5.4: Mean ( \pm standard deviation) organ levels of ${ }^{99 \mathrm{~m}}$ Tc-MIBI, expressed as \% of dose, in each group.

\begin{tabular}{|l|cc|cc|cc|}
\cline { 2 - 8 } \multicolumn{1}{c|}{} & \multicolumn{2}{c|}{ Control } & \multicolumn{2}{c|}{ Solutol HS15 } & \multicolumn{2}{c|}{ Tween 80 } \\
\hline Carcass & 57.36 & $( \pm 3.69)$ & 63.39 & $( \pm 6.12)$ & 62.68 & $( \pm 4.91)$ \\
\hline Liver & 6.64 & $( \pm 1.38)$ & 6.80 & $( \pm 1.79)$ & 7.28 & $( \pm 0.41)$ \\
\hline Kidneys & 10.54 & $( \pm 1.04)$ & 9.85 & $( \pm 1.43)$ & 8.48 & $( \pm 3.46)$ \\
\hline Heart & 2.13 & $( \pm 0.17)$ & 2.30 & $( \pm 0.34)$ & 2.39 & $( \pm 0.44)$ \\
\hline Lungs & 0.85 & $( \pm 0.06)$ & 0.98 & $( \pm 0.22)$ & 0.74 & $( \pm 0.15)$ \\
\hline Stomach & 1.35 & $( \pm 0.32)$ & 1.47 & $( \pm 0.80)$ & 1.40 & $( \pm 0.20)$ \\
\hline Spleen & 0.95 & $( \pm 0.21)$ & 0.98 & $( \pm 0.41)$ & 0.68 & $( \pm 0.24)$ \\
\hline Urine & - & - & - & - & 1.37 & $( \pm 2.06)$ \\
\hline Small intestine & 21.78 & $( \pm 1.81)$ & 14.58 & $( \pm 2.60)$ & 16.08 & $( \pm 1.34)$ \\
\hline Large intestine & 3.64 & $( \pm 0.79)$ & 2.38 & $( \pm 0.42)$ & 2.40 & $( \pm 0.39)$ \\
\hline Total & 106.13 & $( \pm 2.30)$ & 103.04 & $( \pm 2.48)$ & 103.49 & $( \pm 3.03)$ \\
\hline
\end{tabular}


Statistical comparisons between groups for the small and large intestine, and the intestine as a whole, are shown in Figures 5.2, 5.3, and 5.4, respectively. There were no significant differences between any of the groups for the carcass, liver, kidneys, heart, lungs, stomach, spleen, or ratio of \% dose in the large intestine:small intestine. Data for urine was not analysed, as it did not contain sufficient activity to be visible for some rats in the control and Solutol groups.

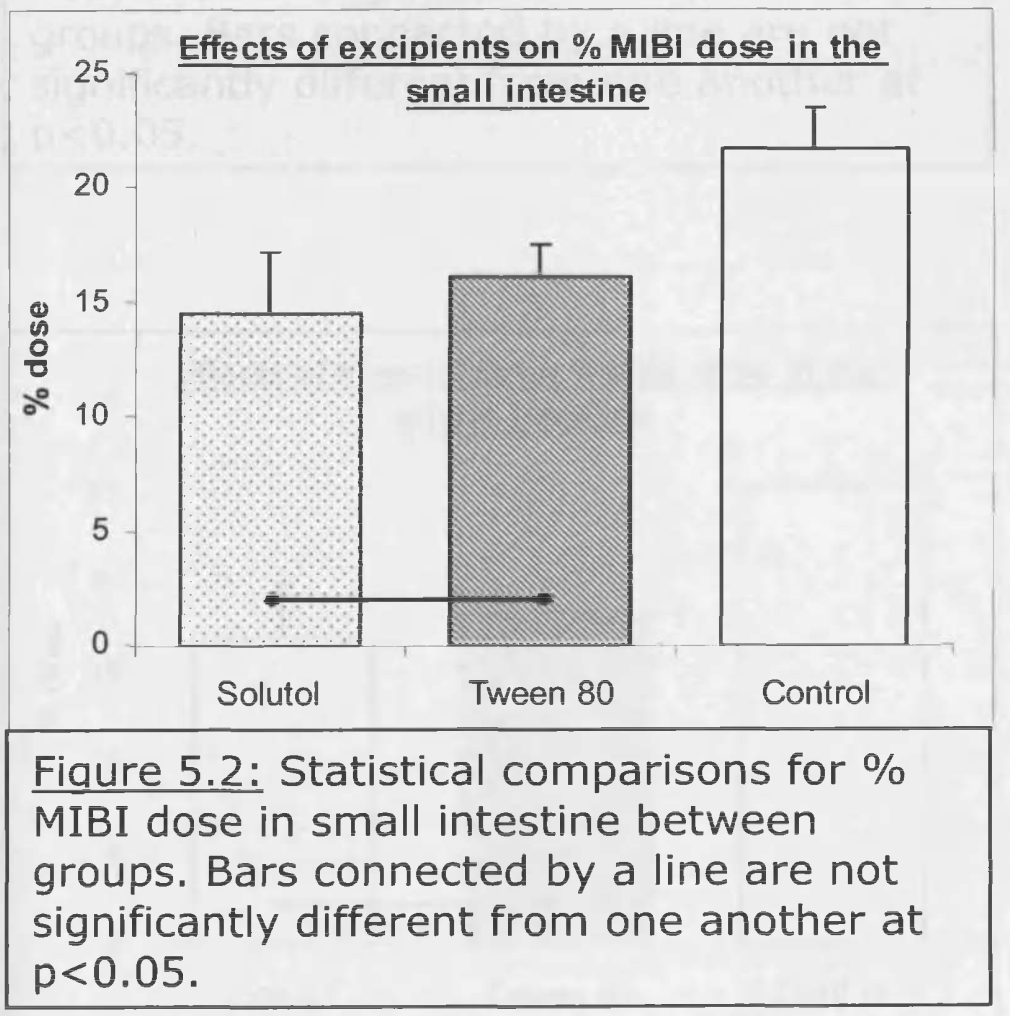



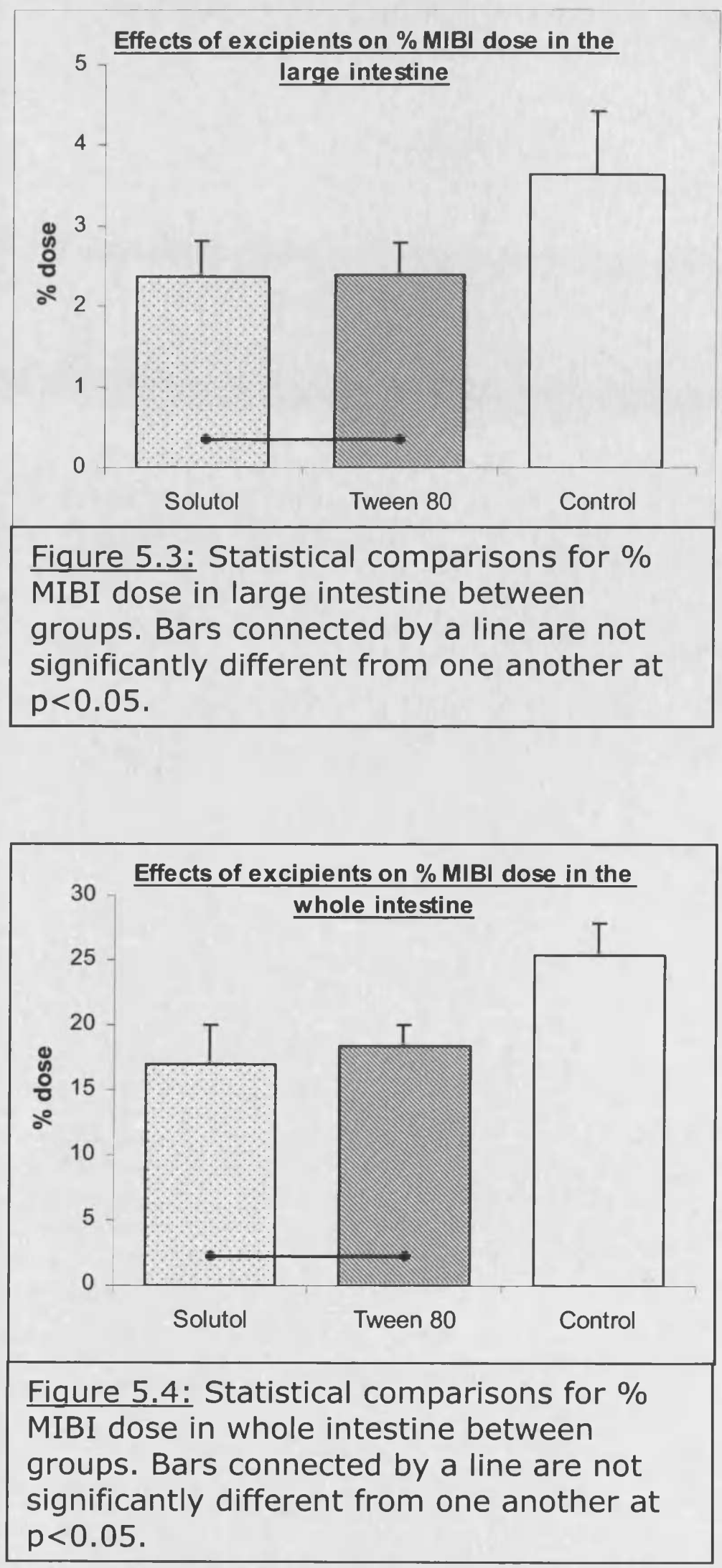


\subsection{2 ${ }^{99 \mathrm{~m}}$ TC-HIDA}

Figure 5.5 shows the time course of ${ }^{99 \mathrm{~m}}$ Tc-HIDA disposition for a typical control rat. ${ }^{99 m}$ Tc-HIDA levels in each organ, expressed as \% of dose, for control, Solutol and Tween groups are shown in Tables 5.5-5.7. After dissection, only the carcass, liver, kidneys, stomach and small intestine contained sufficient activity to be visible on the camera images for rats in the control and Tween 80 groups. Pretreatment with Solutol resulted in increased activity in the urine and lungs of some of the rats in this group, making these also visible on the scintigraphic images. As was seen for ${ }^{99 m}$ Tc-MIBI, the activity in blood was so low that it was sometimes difficult to discern exactly where the ROI should be placed, so no statistical analysis was performed on the blood data. No differences could be seen in the in vivo scintigraphic images between groups.

Due to difficulty in injecting, two of the rats received ${ }^{99 \mathrm{~m}} \mathrm{Tc}-\mathrm{HIDA}$ later than the others in their group in relation to the excipient injection. Rat $D$ in the Solutol group received ${ }^{99 m}$ Tc-HIDA 8.8 minutes after excipient dosing, and rat $\mathrm{A}$ in the Tween group received ${ }^{99 m}$ Tc-HIDA 11.1 minutes after excipient dosing. There was also a small amount of the excipient dose lost when injecting Rat $D$ in the Tween group; approximately $0.01 \mathrm{ml}$ of the $0.22 \mathrm{ml}$ excipient 
dose ran back out of the vein. This rat therefore received

approximately $95 \%$ of the intended Tween dose. 


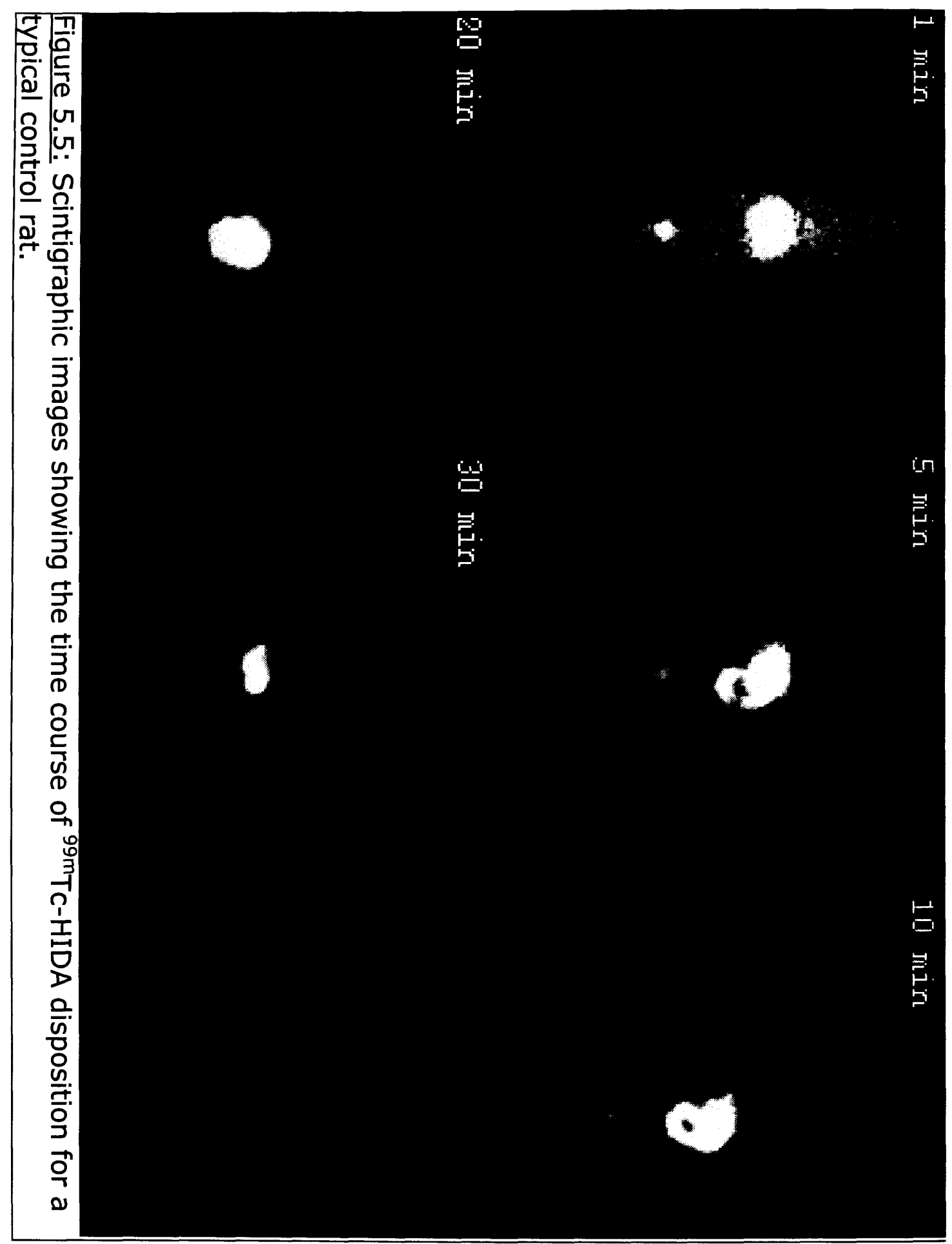




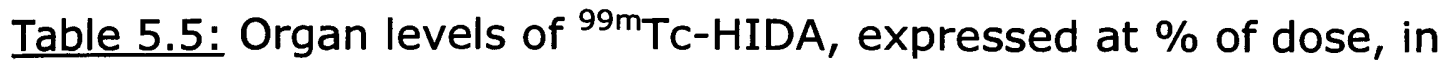
control rats.

\begin{tabular}{|l|c|c|c|c|cc|}
\cline { 2 - 8 } \multicolumn{1}{c|}{} & Rat A & Rat B & Rat C & Rat D & \multicolumn{2}{c|}{ Mean ( \pm SD) } \\
\hline Blood (per ml) & 0.02 & 0.05 & 0.05 & 0.08 & - & \\
\hline Carcass & 4.85 & 8.25 & 5.44 & 14.60 & 8.29 & $( \pm 4.46)$ \\
\hline Liver & 1.79 & 1.73 & 1.33 & 4.02 & 2.22 & $( \pm 1.22)$ \\
\hline Kidneys & 3.59 & 1.14 & 7.23 & 2.52 & 3.62 & $( \pm 2.61)$ \\
\hline Heart & $<0.01$ & $<0.01$ & $<0.01$ & $<0.01$ & - & \\
\hline Lungs & $<0.01$ & $<0.01$ & $<0.01$ & $<0.01$ & - & \\
\hline Stomach & 1.80 & 6.70 & 4.18 & 0.89 & 3.39 & $( \pm 2.60)$ \\
\hline Spleen & $<0.01$ & $<0.01$ & $<0.01$ & $<0.01$ & - & \\
\hline Urine & $<0.01$ & $<0.01$ & $<0.01$ & $<0.01$ & - & \\
\hline Small intestine & 100.06 & 94.50 & 93.56 & 95.43 & 95.89 & $( \pm 2.88)$ \\
\hline Large intestine & $<0.01$ & $<0.01$ & $<0.01$ & $<0.01$ & - & \\
\hline Total & 112.09 & 112.33 & 111.74 & 117.45 & 113.40 & $( \pm 2.71)$ \\
\hline
\end{tabular}

Table 5.6: Organ levels of ${ }^{99 \mathrm{~m}} \mathrm{Tc}-\mathrm{HIDA}$, expressed at $\%$ of dose, in rats pre-treated with Solutol HS15.

\begin{tabular}{|l|c|c|c|c|cc|}
\cline { 2 - 8 } \multicolumn{1}{c|}{} & Rat A & Rat B & Rat C & Rat D & \multicolumn{2}{c|}{ Mean ( \pm SD) } \\
\hline Blood (per ml) & 0.06 & 0.06 & 0.06 & 0.05 & - & \\
\hline Carcass & 10.92 & 15.14 & 8.08 & 9.22 & 10.84 & $( \pm 3.09)$ \\
\hline Liver & 4.38 & 4.37 & 2.73 & 3.22 & 3.68 & $( \pm 0.83)$ \\
\hline Kidneys & 10.15 & 10.24 & 18.22 & 14.86 & 13.37 & $( \pm 3.91)$ \\
\hline Heart & $<0.01$ & $<0.01$ & $<0.01$ & $<0.01$ & - & \\
\hline Lungs & 0.10 & $<0.01$ & 0.07 & 0.09 & - & \\
\hline Stomach & 10.54 & 1.25 & 18.19 & 16.24 & 11.55 & $( \pm 7.60)$ \\
\hline Spleen & $<0.01$ & $<0.01$ & $<0.01$ & $<0.01$ & - & \\
\hline Urine & $<0.01$ & $<0.01$ & 0.37 & 0.31 & - & \\
\hline Small intestine & 76.79 & 78.61 & 64.12 & 67.84 & 71.84 & $( \pm 6.98)$ \\
\hline Large intestine & $<0.01$ & $<0.01$ & $<0.01$ & $<0.01$ & - & \\
\hline Total & 112.87 & 109.61 & 111.78 & 111.77 & 111.51 & $( \pm 1.37)$ \\
\hline
\end{tabular}


Table 5.7: Organ levels of ${ }^{99 \mathrm{~m}} \mathrm{Tc}-\mathrm{HIDA}$, expressed at $\%$ of dose, in rats pre-treated with Tween 80.

\begin{tabular}{|l|c|c|c|c|cc|}
\cline { 2 - 7 } \multicolumn{1}{c|}{} & Rat A & Rat B & Rat C & Rat D & \multicolumn{2}{c|}{ Mean ( \pm SD) } \\
\hline Blood (per ml) & 0.07 & 0.05 & 0.04 & 0.07 & - & \\
\hline Carcass & 8.82 & 5.39 & 4.54 & 10.15 & 7.22 & $( \pm 2.69)$ \\
\hline Liver & 3.25 & 1.95 & 0.99 & 2.37 & 2.14 & $( \pm 0.94)$ \\
\hline Kidneys & 3.65 & 7.97 & 6.77 & 2.46 & 5.21 & $( \pm 2.58)$ \\
\hline Heart & $<0.01$ & $<0.01$ & $<0.01$ & $<0.01$ & - & \\
\hline Lungs & $<0.01$ & $<0.01$ & $<0.01$ & $<0.01$ & - & \\
\hline Stomach & $<0.01$ & 6.93 & 0.46 & 1.17 & - & \\
\hline Spleen & $<0.01$ & $<0.01$ & $<0.01$ & $<0.01$ & - & \\
\hline Urine & $<0.01$ & $<0.01$ & $<0.01$ & $<0.01$ & - & \\
\hline Small intestine & 89.57 & 86.17 & 98.64 & 95.72 & 92.52 & $( \pm 5.68)$ \\
\hline Large intestine & $<0.01$ & $<0.01$ & $<0.01$ & $<0.01$ & - & \\
\hline Total & 105.29 & 108.41 & 111.40 & 111.87 & 107.10 & $( \pm 3.05)$ \\
\hline
\end{tabular}

A comparison of mean organ levels of ${ }^{99 m}$ Tc-HIDA in each group is shown in Table 5.8.

Table 5.8: Mean ( \pm standard deviation) organ levels of ${ }^{99 \mathrm{~m}} \mathrm{Tc}$-HIDA, expressed as \% of dose, in each group.

\begin{tabular}{|l|cc|cc|cc|}
\cline { 2 - 8 } \multicolumn{1}{c|}{} & \multicolumn{2}{c|}{ Control } & \multicolumn{2}{c|}{ Solutol HS15 } & \multicolumn{2}{c|}{ Tween 80 } \\
\hline Carcass & 8.29 & $( \pm 4.46)$ & 10.84 & $( \pm 3.09)$ & 7.22 & $( \pm 2.69)$ \\
\hline Liver & 2.22 & $( \pm 1.22)$ & 3.68 & $( \pm 0.83)$ & 2.14 & $( \pm 0.94)$ \\
\hline Kidneys & 3.62 & $( \pm 2.61)$ & 13.37 & $( \pm 3.91)$ & 5.21 & $( \pm 2.58)$ \\
\hline Stomach & 3.39 & $( \pm 2.60)$ & 11.55 & $( \pm 7.60)$ & - & \\
\hline Small intestine & 95.89 & $( \pm 2.88)$ & 71.84 & $( \pm 6.98)$ & 92.52 & $( \pm 5.68)$ \\
\hline Total & 113.40 & $( \pm 2.71)$ & 111.51 & $( \pm 1.37)$ & 107.10 & $( \pm 3.05)$ \\
\hline
\end{tabular}

Statistical comparisons between groups for the kidneys and small intestine are shown in Figures 5.6 and 5.7, respectively. As variances in the small intestine data were heterogeneous according to Levene's test, the Games-Howell test was used to assess 
significance for data from this organ. There were no significant differences among any of the groups for the carcass, liver, or stomach. Data for lungs and urine were not analysed, as these organs had not contained sufficient activity to be visible in the control group. Data for the stomach were also not analysed for the Tween 80 group, as this organ did not contain sufficient activity to be visible for one rat.

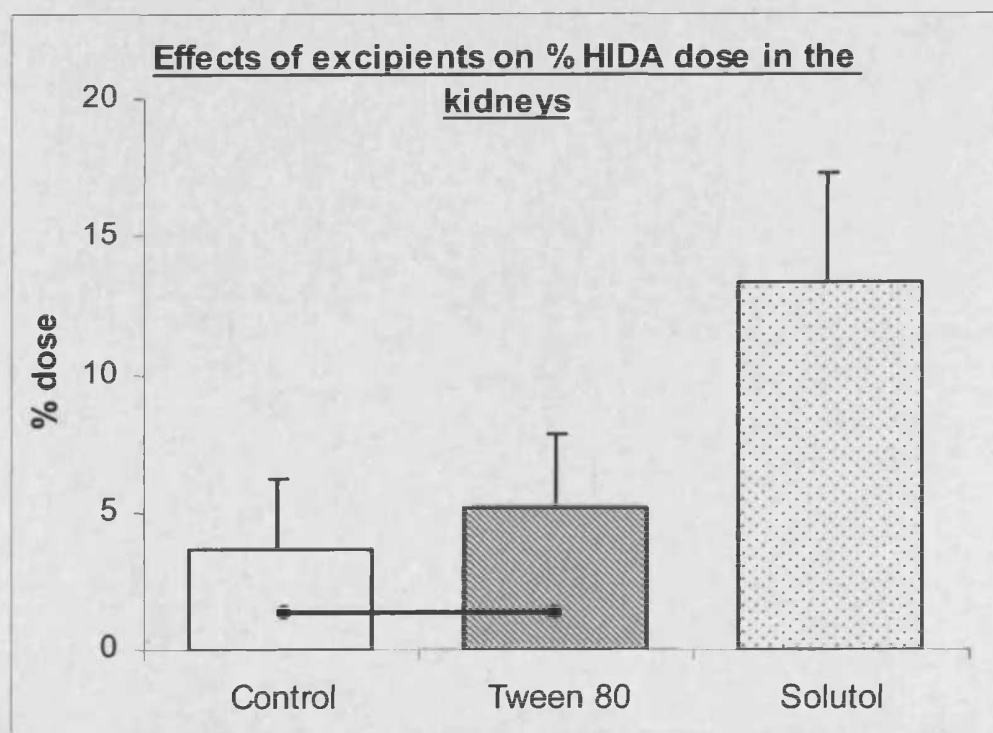

Figure 5.6: Statistical comparisons for \% HIDA dose in kidneys between groups. Bars connected by a line are not significantly different from one another at $p<0.05$. 


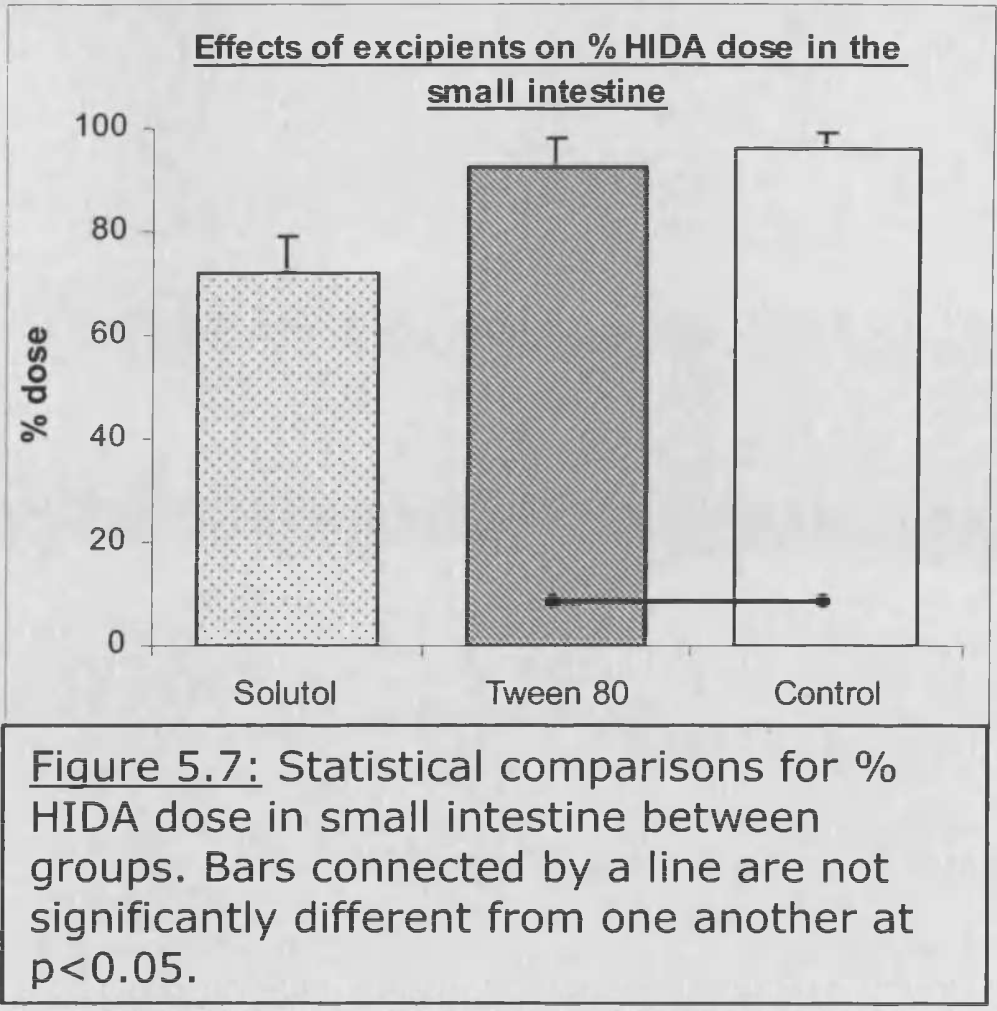

\subsubsection{Effect of injection site retention}

For many of the rats, some of the radioactive dose was initially retained at the injection site. In some cases, some of this dose was then absorbed into the system during the imaging period. This was taken into account when calculating the dose as described in the methods section. However, it is possible that this would happen in some groups to a greater extent than others. It is important to eliminate any potential effects of this on the results seen. Tables 5.9 and 5.10 show the ratio of initial dose:final dose (i.e. total body counts in the first image minus the injection site retention from the first or last image, respectively) for rats receiving ${ }^{99 \mathrm{~m}} \mathrm{Tc}-\mathrm{MIBI}$ and 
${ }^{99 m}$ Tc-HIDA. There were no significant differences between any of the groups treated with either of the agents. This suggests that differences in dose movement from the injection site are not responsible for the differences in disposition seen.

Table 5.9: Ratio of initial to final dose for rats receiving ${ }^{99 \mathrm{~m}} \mathrm{Tc}$-MIBI.

\begin{tabular}{|l|c|c|c|c|cc|}
\cline { 2 - 7 } \multicolumn{1}{c|}{} & Rat A & Rat B & Rat C & Rat D & \multicolumn{2}{c|}{ Mean ( \pm SD) } \\
\hline Control & 0.99 & 1.00 & 0.99 & 0.99 & $0.99( \pm 0.004)$ \\
\hline Solutol HS15 & 0.96 & 0.84 & 1.00 & 1.00 & 0.95 & $( \pm 0.077)$ \\
\hline Tween 80 & 0.97 & 0.98 & 0.97 & 0.86 & $0.95 \quad( \pm 0.057)$ \\
\hline
\end{tabular}

Table 5.10: Ratio of initial to final dose for rats receiving ${ }^{99 \mathrm{~m}} \mathrm{Tc}$ HIDA.

\begin{tabular}{|l|c|c|c|c|cc|}
\cline { 2 - 7 } \multicolumn{1}{c|}{} & Rat A & Rat B & Rat C & Rat D & \multicolumn{2}{c|}{ Mean ( \pm SD) } \\
\hline Control & 0.86 & 0.96 & 0.97 & 0.75 & 0.89 & $( \pm 0.107)$ \\
\hline Solutol HS15 & 0.98 & 0.69 & 0.97 & 0.99 & 0.91 & $( \pm 0.144)$ \\
\hline Tween 80 & 0.73 & 0.79 & 0.98 & 0.97 & 0.87 & $( \pm 0.124)$ \\
\hline
\end{tabular}




\subsection{Discussion}

Pre-treatment with $160 \mathrm{mg} / \mathrm{kg}$ Solutol HS15 resulted in significantly lower ${ }^{99 \mathrm{~m}}$ Tc-MIBI activity in the small and large intestines. The $\%$ of ${ }^{99 m}$ Tc-MIBI dose in the small intestine was reduced by $33 \%$, and that in the large intestine by $35 \%$. This suggests a reduction in the biliary elimination of ${ }^{99 \mathrm{~m}} \mathrm{Tc}$-MIBI. There was also a small but nonsignificant increase in carcass levels $(11 \%)$, which would be consistent with inhibited elimination of ${ }^{99 \mathrm{~m}}$ Tc-MIBI. There were no apparent compensatory increases in ${ }^{99 \mathrm{~m}}$ Tc-MIBI levels for other routes of elimination, such as the kidneys. As ${ }^{99 \mathrm{~m}} \mathrm{Tc}-\mathrm{MIBI}$ is a substrate for P-Gp and MRP (Hendrikse et al. 2004; Hendrikse et al. 1998), inhibition of either or both of these transport proteins by Solutol could be responsible for the changes seen. The fact that the differences between groups remained significant when counts for the whole intestine were compared, and the ratio of counts in the small:large intestine were not significantly different, suggests that Solutol did not affect intestinal transit.

In the ${ }^{99 \mathrm{~m}} \mathrm{Tc}$-HIDA studies, pre-treatment with Solutol resulted in a $25 \%$ reduction in the $\%$ dose in the small intestine, and a 3.7-fold increase in the \% dose in the kidneys. Additionally, the lungs of three rats and the urine of two rats were visible on the postdissection images. As these were not visible for any of the rats in 
the control group, this demonstrates an increase in ${ }^{99 m}$ Tc-HIDA activity in these organs due to the excipient, although it was not possible to perform statistical analysis on these data. Levels of ${ }^{99 \mathrm{~m}} \mathrm{Tc}$-HIDA also appeared to increase in the carcass (31\%), stomach (3.4-fold) and liver (66\%) in the Solutol group, but these changes were not significant, possibly due to large standard deviations. The reduction in activity in the small intestine, coupled with the increased activity in the kidney, appears to demonstrate a change in the differential renal and biliary excretion of ${ }^{99 m}$ Tc-HIDA. It is not possible to tell from the data which of the changes seen are cause and which are effect, i.e. whether the increase in renal activity is due to reduced biliary elimination, or biliary elimination was reduced by an increase in renal elimination. However, when considered together with the apparent effect of Solutol on biliary elimination of ${ }^{99 \mathrm{~m}} \mathrm{Tc}-\mathrm{MIBI}$, a reduction in biliary elimination causing the increased renal counts seems to be the most likely explanation.

As ${ }^{99 \mathrm{~m}}$ Tc-HIDA as a substrate for MRP2 (Hendrikse et al. 2004), inhibition of this transport protein by the excipient is the likely mechanism for the reduction in biliary elimination seen. The effects seen with ${ }^{99 m}$ Tc-MIBI above could be due to either inhibition of MRP2 alone, which has been shown to inhibit biliary secretion of ${ }^{99 m}$ Tc-MIBI (Hendrikse et al. 2004), or inhibition of both MRP2 and P-Gp. In the in vitro studies described in the previous chapter, 
Solutol significantly increased the A-B transport of ${ }^{3} \mathrm{H}$-vinblastine and ${ }^{99 \mathrm{~m}}$ Tc-MIBI across MDCK MDR1 cells, consistent with inhibition of P-Gp. As discussed in Chapter 4 other authors have also reported P-Gp inhibition by this excipient. Although inhibition of P$\mathrm{Gp}$ in vitro does not necessarily confirm that this is the mechanism in vivo, it seems likely that the effects of Solutol on ${ }^{99 m}$ Tc-MIBI biliary elimination could be due to inhibition of both P-Gp and MRP2.

There is one report in the literature which suggests that Solutol may have impaired biliary elimination in vivo by inhibition of $\mathrm{P}-\mathrm{Gp}$. Bittner et al. (2003b) reported that Solutol reduced colchicine CL in rats by $55 \%$, and increased $C_{\max } 2.4$-fold. No significant alteration in $t_{1 / 2} S$ was seen. $V$ was reduced by $58 \%$, but this was not significant. The excipient also caused a 35 -fold increase in the amount of colchicine excreted in urine. The authors concluded that Solutol had altered metabolism and distribution processes; inhibited metabolism was suggested as a likely cause, as Solutol also reduced the in vitro $\mathrm{CL}$ of colchicine in rat hepatocytes, at concentrations of 0.003\% and above. However, when Desrayaud et al. (1997) investigated the effects of the P-Gp inhibitor SDZ PSC 833 on colchicine pharmacokinetics, similar effects were seen. $\mathrm{AUC}_{0-6 \mathrm{~h}}$ was increased by 2.5 -fold and $C_{\max }$ by 2.2 -fold, while $C L$ was reduced by $47 \% . V_{\beta}$ was reduced by $18 \%$, which was not significant. This suggests that the effects seen in Bittner et al.'s study could be due, 
at least partially, to P-Gp inhibition by Solutol. This would fit in with the inhibition of biliary elimination of ${ }^{99 \mathrm{~m}} \mathrm{Tc}$-MIBI seen in the present study, although this may have been due to MRP2 alone.

However, it is possible that other mechanisms are responsible for the changes seen with Solutol in the present study. It is possible that the effects seen here are the result of a non-specific effect of Solutol on biliary elimination which is not related to $A B C$ transporters, such as a general reduction in bile flow. However, the fact that this excipient inhibited P-Gp-mediated ${ }^{99 \mathrm{~m}} \mathrm{Tc}-\mathrm{MIBI}$ transport in the in vitro experiments described in the previous chapter suggests that this may be the most likely mechanism. There were no significant differences in liver levels of ${ }^{99 \mathrm{~m}} \mathrm{Tc}$-MIBI or ${ }^{99 \mathrm{~m}}$ Tc-HIDA between groups, nor was any non-significant reduction in liver levels apparent in the Solutol treatment groups. This suggests that the reduction in the amount appearing in the intestine is not due to Solutol restricting the entry of ${ }^{99 \mathrm{~m}} \mathrm{Tc}-\mathrm{MIBI}$ or ${ }^{99 \mathrm{~m}} \mathrm{Tc}-$ HIDA into the liver, for example by micellar entrapment.

Solutol HS15 is commonly used in pre-clinical studies at doses up to $10 \% 3 \mathrm{ml} / \mathrm{kg}$, equivalent to $300 \mathrm{mg} / \mathrm{kg}$ (M. DeMatas, personal communication). The present experiments have demonstrated a significant effect of the excipient on biliary elimination at a dose lower than this; it is possible that a greater effect may be seen 
when higher doses are used. The effects described here are therefore relevant to early pharmacokinetic studies, and could potentially affect the conclusions drawn from such studies.

Tween 80 caused a significant reduction in the $\%$ dose of ${ }^{99 m} \mathrm{Tc}-$ MIBI found in the small (26\%) and large intestine (34\%). This suggests a reduction in biliary elimination. These effects were not significantly different from those of Solutol. As seen for Solutol, no effects of the excipient on intestinal transit were apparent, demonstrated by the fact that the differences between groups remained significant when counts for the whole intestine were compared, and the ratio of counts in the small: large intestine was not significantly different between groups. There was also a small but non-significant increase in the carcass counts (9\%), which would fit in with reduced overall elimination of ${ }^{99 \mathrm{~m}}$ Tc-MIBI.

As ${ }^{99 \mathrm{~m}}$ Tc-MIBI is a substrate for both P-Gp and MRP (Hendrikse et al. 2004; Hendrikse et al. 1998), these effects could potentially have been caused by inhibition of either transport protein. However, a clearer picture emerges upon examination of the ${ }^{99 \mathrm{~m}} \mathrm{Tc}-\mathrm{HIDA}$ data. As ${ }^{99 m}$ Tc-HIDA is an MRP2 substrate (Hendrikse et al. 2004), any effects on MRP2 would also have been expected to affect it's disposition. The lack of significant effect of Tween on the disposition of ${ }^{99 \mathrm{~m}} \mathrm{Tc}-\mathrm{HIDA}$ therefore suggests that the effects seen in the ${ }^{99 \mathrm{~m}} \mathrm{Tc}-$ 
MIBI studies are due to inhibition of P-Gp, rather than MRP2. The ${ }^{99 m}$ Tc-HIDA data also suggest that the apparent reduction in biliary elimination seen for ${ }^{99 m}$ Tc-MIBI is not due to a non-specific effect of Tween on biliary elimination, such as a reduction in hepatic blood flow, as this would have been expected to affect the disposition of both compounds.

As discussed in the previous chapter, Tween 80 significantly increased the A-B transport of ${ }^{3} \mathrm{H}$-vinblastine across MDCK MDR1 cells, and caused a non-significant 4.4 -fold increase in ${ }^{99 \mathrm{~m}}$ Tc-MIBI A-B transport across these cells. This demonstrates that the excipient can inhibit the P-Gp mediated transport of ${ }^{99 \mathrm{~m}} \mathrm{Tc}-\mathrm{MIBI}$, and provides further evidence that this is the likely mechanism for the observed reduction in biliary elimination.

As discussed in Chapter 4, there are several reports in the literature describing P-Gp inhibition by Tween 80 . Additionally, there are studies which specifically suggest that this excipient can inhibit the biliary elimination of P-Gp substrates. Ellis et al. (1996) reported that Tween 80 impaired the biliary elimination of etoposide in the isolated perfused rat liver. The excipient reduced the rate constant for biliary excretion by $29 \%$, although the total amount of etoposide eliminated in bile was not affected, as the preparation appeared to recover from this effect over time. Tween also increased perfusate 
concentrations of the drug, significantly reduced $\mathrm{CL}(38 \%), \mathrm{V}$ $(66 \%)$, and $t_{1 / 2}(44 \%)$, and appeared to change the perfusate elimination profile from biphasic to monophasic. However, these effects may also have been due to decreased or delayed liver uptake due to drug entrapment in micelles. Tween 80 also appeared to reduce bile flow, although this was only significant at a single timepoint (180 minutes). However, such an effect does not appear to be responsible for the effects of Tween on ${ }^{99 m}$ Tc-MIBI biliary elimination in the present studies, as discussed above. Zhang et al. (2003) reported that Tween $80(10 \% 1 \mathrm{ml} / \mathrm{kg}$, equivalent to $106 \mathrm{mg} / \mathrm{kg}$ ) caused a $36 \%$ increase in digoxin AUC and a $24 \%$ reduction in $C L$ in rats. This was attributed to inhibition of $P$ Gp-mediated billiary and renal efflux of the drug.

Tween is usually used in early pharmacokinetic studies at doses up to $5 \% 5 \mathrm{ml} / \mathrm{kg}$, equivalent to $266 \mathrm{mg} / \mathrm{kg}$ (M. DeMatas, personal communication). The present study has shown effects on biliary elimination at a dose of Tween 80 lower than this $(85 \mathrm{mg} / \mathrm{kg})$, demonstrating that the effects seen here could occur in pre-clinical studies. It is possible that this effect would be increased at higher doses of Tween.

As described above, significant effects on the disposition of ${ }^{99 \mathrm{~m}} \mathrm{Tc}-$ MIBI and ${ }^{99 m}$ Tc-HIDA were shown using the dissection data. 
However, no differences between the groups could be discerned from the in vivo scintigraphic images. It appears that, as discussed in the introduction, the changes caused by the excipients were too small to visualise in this way, due to overlaying of organs on the images. However, the scintigraphic images were still useful for calculation of the dose received by each rat.

The sum total of the radioactivity contained in organs, carcass and urine are in most cases greater than $100 \%$, and are especially high for the ${ }^{99 m}$ Tc-HIDA experiments. This is due to the effects of tissue attenuation. In the in vivo image used to calculate the dose (i.e. $100 \%)$, the gamma radiation will be attenuated by the tissues of the rat, varying in extent with the distance and type of tissue that it has to pass through to reach the camera. However, after dissection, the organs, where most of the radioactivity was contained, were imaged outside of the rat. This would reduce the attenuation, as the attenuation provided by the plastic dishes on which the organs were imaged will be very small compared to that provided by tissues in vivo. Thus, the total number of counts which can be detected from the same amount of radioactivity is increased when organs are removed from the body. Using the last image to calculate the dose demonstrates this to a certain extent. During the imaging period, some of the dose was eliminated from the systemic circulations via bile (and urine for some rats). As the intestine and bladder are near the front of the 
rat, the radioactivity located here has reduced attenuation in anterior images taken by the camera. Use of the corrected counts in the last image as the 'dose' for calculation purposes reduced the average sum of post-dissection radioactivity from $113.4 \%$ to $108.0 \%$ for the ${ }^{99 m}$ Tc-HIDA control group, and from $111.5 \%$ to $107.0 \%$ for the ${ }^{99 \mathrm{~m}} \mathrm{Tc}-\mathrm{HIDA}$ Solutol treatment group, demonstrating that lower attenuation results in a mass balance closer to $100 \%$. However this cannot realistically be used as an estimate of dose, as rats may have excreted some radioactivity from the body entirely during the dosing period. Also, it is not sensible to use the total radioactivity detected after dissection as an indication of dose, as again some of the radioactivity may have been excreted by the rat between images, so this is not a reliable indicator of the dose of radioactivity a rat received. In the present study, it is the differences between control and excipient treatment groups which are of importance. The fact that the mass balance is slightly greater than $100 \%$ does not prevent comparison of these data.

\subsubsection{Conclusions}

The present studies have demonstrated that two commonly used pharmaceutical excipients can significantly impair the biliary elimination of ${ }^{99 \mathrm{~m}} \mathrm{Tc}-\mathrm{MIBI}$ and ${ }^{99 \mathrm{~m}} \mathrm{Tc}-\mathrm{HIDA}$, at doses which would be 
reasonable to use in pre-clinical studies. These changes appear to be due to inhibition of P-Gp by Tween, and inhibition of MRP2 and possibly P-Gp by Solutol. The potential of these excipients to affect disposition in this way should therefore be borne in mind when selecting excipients for use in pre-clinical studies, as the effects seen here could mask the drug's true pharmacokinetic profile.

\subsubsection{Further work}

It would be of interest to further probe these effects by carrying out in vitro work with ${ }^{99 m} \mathrm{Tc}$-HIDA, in cell lines over-expressing MRP1 and 2. However, due to time and resource limitations, this was not possible. It would also be of interest to ascertain whether the effects of the excipients seen here would be greater at increased doses. 


\section{Chapter 6:}

Effects of excipients on renal elimination 


\section{Contents:}

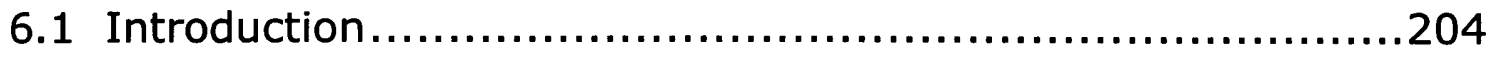

6.2 Methods and materials .......................................211

6.2.1 Materials and equipment .................................211

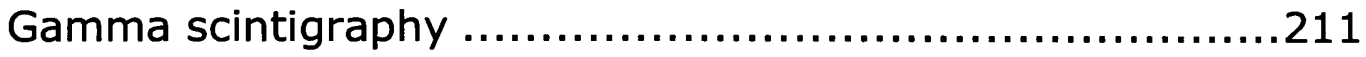

6.2.2 Method development ....................................212

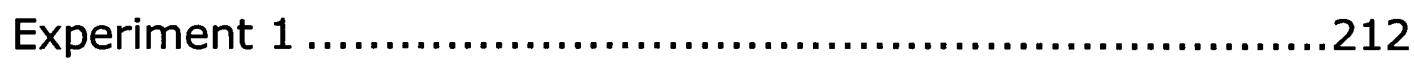

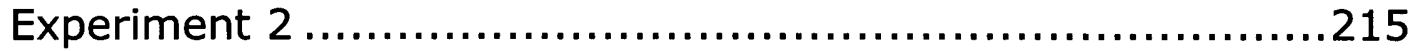

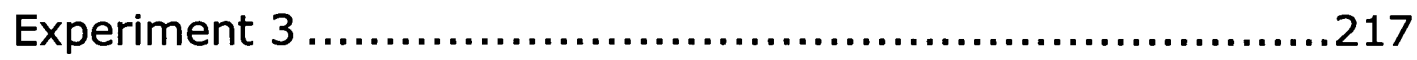

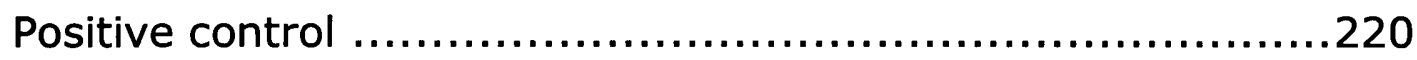

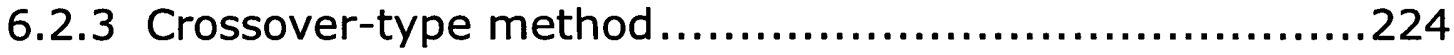

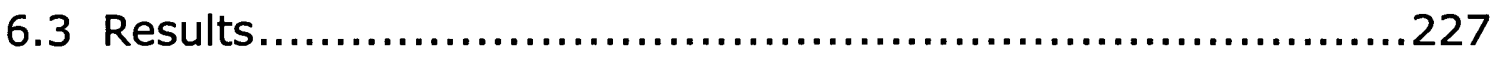

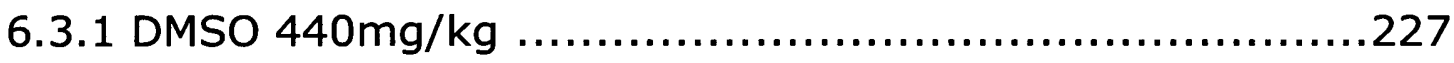

$6.3 .2829 \mathrm{mg} / \mathrm{kg}$ PG ...........................................231

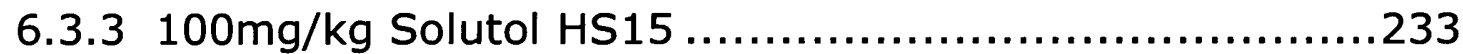

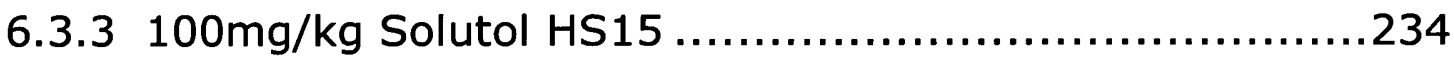

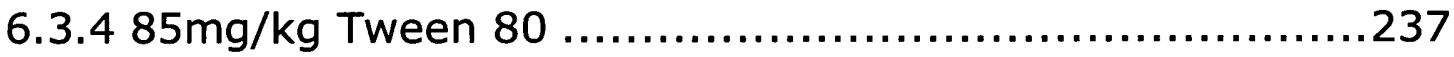

6.3.5 Positive control - 4400mg/kg DMSO ......................240

6.3.6 Comparison of data from control renograms ...............241

6.4 Discussion ..................................................... 242

6.4 .1 Conclusions ................................................ 255

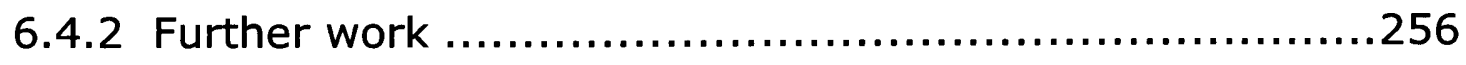




\subsection{Introduction}

The kidney is one of the major eliminating organs in the body, excreting drugs and their metabolites into the urine. The amount of drug excreted in the urine is the net result of several processes. First, blood is filtered at the glomerulus. In humans, plasma water is filtered at a rate of approximately $120-125 \mathrm{ml} / \mathrm{min}$. Drug molecules will pass easily through the filter, and thus be removed from the blood and enter the filtrate. However, larger molecules, such as plasma proteins, will not pass through the filter and so remain in the bloodstream.

From the glomerulus, the filtrate passes into the proximal tubule. Here, active secretion of substances from the blood into the filtrate, and reabsorption from the filtrate into the blood, take place. There are selective transporters for the secretion of acids, bases, and quaternary ammonium compounds, and reabsorption of endogenous compounds such as vitamins, glucose and amino acids. These transporters also transport some drug substances, for example, penicillin is secreted by the organic acid transporter (Lote, 2000a). There are also several $A B C$ transporters present in the kidney epithelium; P-Gp, MRP2, MRP4 and BCRP are found in the apical membrane, and MRP1, 3, and 5 in the basolateral membrane (Schinkel and Jonker, 2003). 
Passive reabsorption also occurs along the whole length of the tubule. The susceptibility of a compound to passive reabsorption depends upon its ability to diffuse across membranes. Passive reabsorption is promoted by the reabsorption of water along the tubule, which increases the concentration of drug in filtrate relative to that in plasma, increasing the concentration gradient for reabsorption. Drug remaining in the filtrate passes down the proximal tubule into the Loop of Henle, the distal tubule, then into the collecting ducts where it passes along the ureters into the bladder to be eliminated in the urine. Thus the amount of drug excreted in the urine depends upon the amount filtered at the glomerulus, the amount reabsorbed by both active and passive processes, and the amount actively secreted.

There are therefore several mechanisms by which an excipient could affect renal elimination of co-administered drugs. It is possible that excipients could inhibit active secretion and reabsorption mechanisms in the kidney, either by a non-competitive interaction with the transporter or surrounding membrane, or simply by competing with drugs and metabolites for transport.

Excipients can also increase renal elimination of drugs through toxic effects at the kidney. This was suggested as a potential mechanism 
for the Solutol HS15-mediated increase in renal elimination of colchicine; the excipient increased $A_{e}$ (0-8 hours) from $1 \%$ to $39 \%$ of the dose (Bittner et al., 2003b). There are also reports of PG causing nephrotoxicity in patients receiving the excipient as an injection vehicle (Hayman et al., 2003; Yaucher et al., 2003).

Excipients could also affect renal elimination by altering glomerular filtration rate (GFR). An increase or decrease in GFR would result in a change in the rate at which drug entered the filtrate. Alteration of GFR is therefore an important mechanism by which excipients could affect drug pharmacokinetics, as it is relevant to all drugs which undergo renal elimination, whereas inhibition of individual transport processes would only affect the pharmacokinetics of drugs which are substrates for these. For drugs which do not undergo any secretion or reabsorption processes, an increase or decrease in GFR would cause a corresponding increase or decrease in the rate at which the drug appeared in the urine, and could alter the total amount excreted in the urine if the drug was also subject to another route of elimination. For drugs which are secreted or reabsorbed, a change in the rate at which drug appeared in the filtrate could potentially alter the equilibrium of these processes.

The gold standard method for measuring GFR is inulin clearance (Leyssac and Christensen, 1995; Lote, 2000b). Inulin is a 
polysaccharide which is neither secreted nor reabsorbed, thus its clearance provides an accurate measure of GFR. This technique is widely used in research. However, it is less suited to very small animals such as the rat, due to the need for either repeated blood sampling or a constant infusion of inulin in order to determine the clearance. Some workers have performed these experiments under surgical conditions with cannulation of blood vessels. However, a wide range of surgical anaesthetic regimens have been shown to significantly reduce renal blood flow in the rat (Gumbleton et al., 1990), which would in turn impair GFR. Chronic catheterisation of the animals would be difficult to perform and require surgical expertise, and has been shown to affect the pharmacokinetics of some drugs (Terao and Shen, 1983; Torres-Molina et al., 1992).

Scintigraphic methods are a non-invasive alternative for measurement of GFR. ${ }^{99 \mathrm{~m}} \mathrm{Tc}-\mathrm{DTPA}$ is used in both clinical and veterinary practice for this purpose (Peters, 1991; Kerl and Cook, 2005). Kerl and Cook (2005) describe an established veterinary method for detemination of GFR using ${ }^{99 \mathrm{~m}}$ Tc-DTPA renal scintigraphy in dogs and cats. Sequential gamma-camera images are taken over the first three minutes after injection. ${ }^{99 \mathrm{~m}}$ Tc-DTPA uptake into kidneys during the early accumulation phase is summed and converted to a percentage of the injected dose, and then to GFR using a published equation. 
Several recent studies have used ${ }^{99 \mathrm{~m}}$ Tc-DTPA in small laboratory animals. The simplest method was described by Donadio et al. (1998). They used the amount of ${ }^{99 m}$ Tc-DTPA excreted into the urine over the first 11 minutes as an indication of GFR. The authors acknowledge that this will not provide an actual measurement of GFR, but reasoned that the amount appearing in the urine will be an index of GFR, suitable for comparing the effects of different treatments on this parameter. They used this method to compare the effects of different contrast media on GFR in rats, and demonstrated a significant reduction in GFR in all treatment groups. This method has the advantage of being simple, but was not evaluated against a conventional measurement of GFR, and the amount of ${ }^{99 m}$ Tc-DTPA in the bladder at 11 minutes will also be dependent on urine flow rate. This method also provides limited information, as no scintigraphic images were taken, and the amount of ${ }^{99 \mathrm{~m}} \mathrm{Tc}-\mathrm{DTPA}$ in the urine was quantified using a scintillation counter. Also, as urine was collected by sacrificing the rats, it would not be suited to crossover experiments.

VandeWiele et al. (1997) used ${ }^{99 m}$ Tc-DTPA in rats to evaluate changes in differential renal function in ureter obstruction. 30 twosecond images were obtained, followed by 190 six second images, making a total imaging time of 20 minutes. ${ }^{99 m}$ Tc-DTPA uptake into 
each kidney between 80 and 120 seconds was summed, and the total for each kidney expressed as a percentage of the total accumulation in both kidneys to obtain the differential renal function. While calculation of differential renal function is not really suited to investigation of excipient effects, this report does demonstrate the possibility of using the summed early kidney uptakes for comparing GFRs.

Provoost and van Aken (1985) performed a functional study in three-kidney rats using ${ }^{99 m}$ Tc-DTPA. 10-second images were taken for three minutes, then 1-minute images for 12 minutes. Counts in each kidney between 30 and 90 seconds were summed, and the percentage contribution of each kidney to GFR calculated by dividing the summed counts in each kidney by the total of summed counts. EDTA clearances were also determined in the rats to measure total GFR; the GFR measured using EDTA was multiplied by the percentage contribution of the individual kidneys to calculate the GFR of each kidney. This enabled them to demonstrate that the GFR of the individual kidneys was reduced in the three-kidney rats so that global GFR was no different to two-kidney rats. While this is an interesting method, it would not be necessary to use all components of it to investigate the effects of excipients on GFR. Comparison of the early uptake of ${ }^{99 \mathrm{~m}} \mathrm{Tc}$-DTPA in the kidneys should suffice for the 
purposes of the present studies, without the need to determine EDTA clearance.

Kopecky et al. (1990) investigated the effects of enalaprilat on ${ }^{99 m}$ Tc-DTPA renograms in rats with induced (clip) renovascular hypertension. 15-second images were collected over 15 minutes using a gamma camera, and the counts in the kidneys in the 30-90 second window were summed and converted to percent of dose. This was then converted to ${ }^{99 \mathrm{~m}} \mathrm{Tc}-\mathrm{DTPA}$ clearance $(\mathrm{ml} / \mathrm{min} / 100 \mathrm{~g})$ using an equation from a previous study, where ${ }^{99 \mathrm{~m}}$ TC-DTPA clearance and renal accumulation had been measured simultaneously. This method is analogous to the established veterinary method for determining GFR using ${ }^{99 m}$ Tc-DTPA. The equation used to convert ${ }^{99 \mathrm{~m}}$ Tc-DTPA accumulation into a clearance value may not be applicable to all strains of rat; however, conversion of the data to a clearance value is not necessary for comparing the effects of different treatments.

It can be seen from the above that a variety of methods for determining changes in GFR using ${ }^{99 m}$ Tc-DTPA have been described in small laboratory animals. This series of experiments aims to develop a suitable scintigraphic method to detect excipient-induced changes in GFR in rats using ${ }^{99 m}$ Tc-DTPA, and to apply this method to investigate the effects of excipients on GFR. 


\subsection{Methods and materials}

\subsubsection{Materials and equipment}

${ }^{99 m}$ Tc-DTPA was obtained from the Medical Physics department at University Hospital Wales, Cardiff, and was used within 6 hrs of preparation. DMSO, Tween 80 and PG were obtained from Fisher, UK. Solutol HS15 was a kind gift from AstraZeneca, Cheshire, UK.

\section{Animals}

Male Wistar rats (215-300g) (Harlan, UK) were group-housed on a 12-hour light-dark cycle, with access to standard laboratory rat chow and water ad libitum.

\section{Gamma scintigraphy}

Scintigraphic images were taken using a General Electric Maxicamera (model $400 \mathrm{~A}$ ) with $\times 1.6$ magnification. Images were processed as described below. 


\subsubsection{Method development}

\section{Experiment 1}

It was uncertain whether the available gamma scintigraphy equipment would give sufficient resolution to image individual kidneys in the rat. Therefore the first experiment aimed to determine bladder accumulation of ${ }^{99 m}$ Tc-DTPA. This would be similar to the method of Donadio et al. (1998) described above, but taking several scintigraphic images would enable us to plot the complete time course of bladder accumulation, making this potentially a more sensitive method.

Animals $(n=4)$ received an i.v. dose of 2-2.5 MBq ${ }^{99 m}$ Tc-DTPA iv. Isoflurane anaesthesia was used to assist with injection. Animals were immediately transferred to the gamma camera. 20 -second posterior images were taken at 1, 2 and 3 minutes post-injection, then 2-4 further 30 second images taken between 4 and 13 minutes post-injection. Regions of interest (ROIs) were defined for each kidney using an early image, and for the bladder using a late image. The ROIs were superimposed on all other images of the animal, except where this was not practical due to the animal changing position significantly between images. Background counts were 
obtained by drawing a ROI in the right hind leg of the animal, ensuring that this ROI did not overlap with the bladder. Counts per pixel per second were calculated for both kidneys and the bladder and corrected for background.

Both kidneys were clearly visible and defined especially in the early images, demonstrating that it would be possible to determine early kidney uptake analogous to the methods described above (Provoost and van Aken, 1985; Kopecky et al., 1990; VandeWiele et al., 1997; Kerl and Cook, 2005). An initial increase in kidney counts over the first 2-3 minutes, followed by an elimination phase was apparent from the images (as shown in Figure 6.1). However, under this imaging protocol not enough images had been obtained, especially at early timepoints, to accurately model renal accumulation and elimination.

Three of the animals urinated between imaging, so bladder accumulation of ${ }^{99 m}$ Tc-DTPA over the whole imaging time could not be determined. Although this possibility had been anticipated it was hoped that urination would not occur during so short a time period. However it was apparent that counts accumulated in the bladder over time (until urination occurred) as the ${ }^{99 m}$ Tc-DTPA was transferred there in urine after filtration at the kidneys. 
It was decided that it would be preferable to use the more accurate method of measuring kidney uptake of ${ }^{99 m}$ Tc-DTPA to assess GFR, now that it had been demonstrated this was possible with our equipment, rather than bladder accumulation.

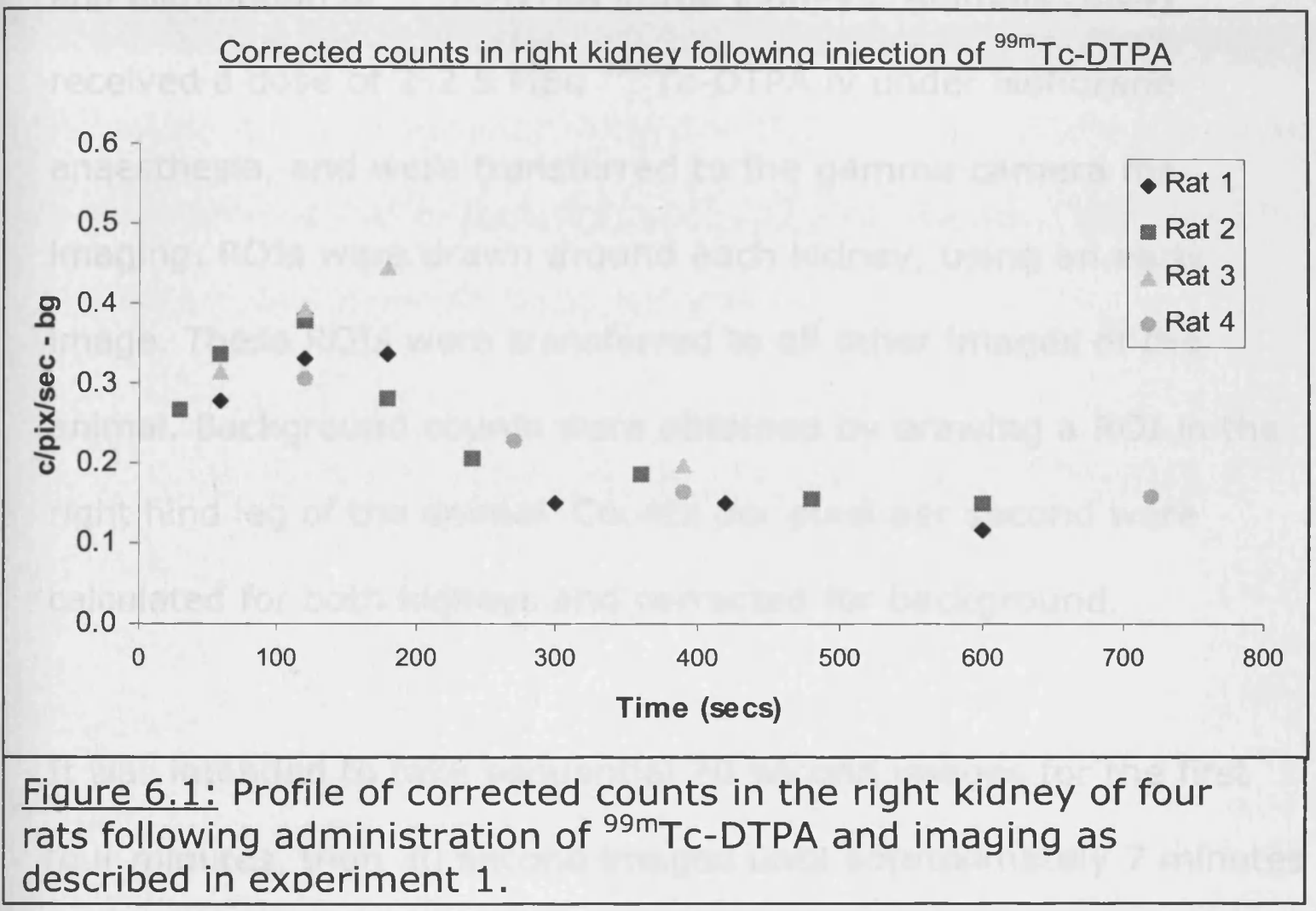




\section{Experiment 2}

The next experiment therefore aimed to obtain a sufficient number of images to more accurately determine the rates of accumulation and elimination of ${ }^{99 m}$ Tc-DTPA in the kidneys. Animals $(n=4)$ received a dose of 2-2.5 $\mathrm{MBq}^{99 \mathrm{~m}}$ Tc-DTPA iv under isoflurane anaesthesia, and were transferred to the gamma camera for imaging. ROIs were drawn around each kidney, using an early image. These ROIs were transferred to all other images of the animal. Background counts were obtained by drawing a ROI in the right hind leg of the animal. Counts per pixel per second were calculated for both kidneys and corrected for background.

It was intended to take sequential 20 second images for the first four minutes, then 30 second images until approximately 7 minutes post-injection, using a restraining device in order to keep the animals still when they revived following the anaesthesia. However it became apparent that the restraining device did not sufficiently immobilise the animals to enable imaging. The time taken to reanaesthetise the animals with isoflurane to enable imaging lead to large gaps of about 120 seconds in the sequence of images. This lead to loss of data in the elimination phase of the plot. Figure 6.2 shows the corrected counts in the right kidney for three of the rats 
(one had been excluded from the analysis, as ${ }^{99 \mathrm{~m}}$ Tc-DTPA was initially retained at the injection site and then appeared to enter the bloodstream at around four minutes post-injection, leading to a second accumulation phase in the kidneys which compromised the data). The initial accumulation phase and the following elimination phase and plateau are clearly visible upon examination of the plot. However, it is also apparent from the plot that we still did not obtain a sufficient number of early data points to sufficiently characterise the accumulation phase in the kidneys.

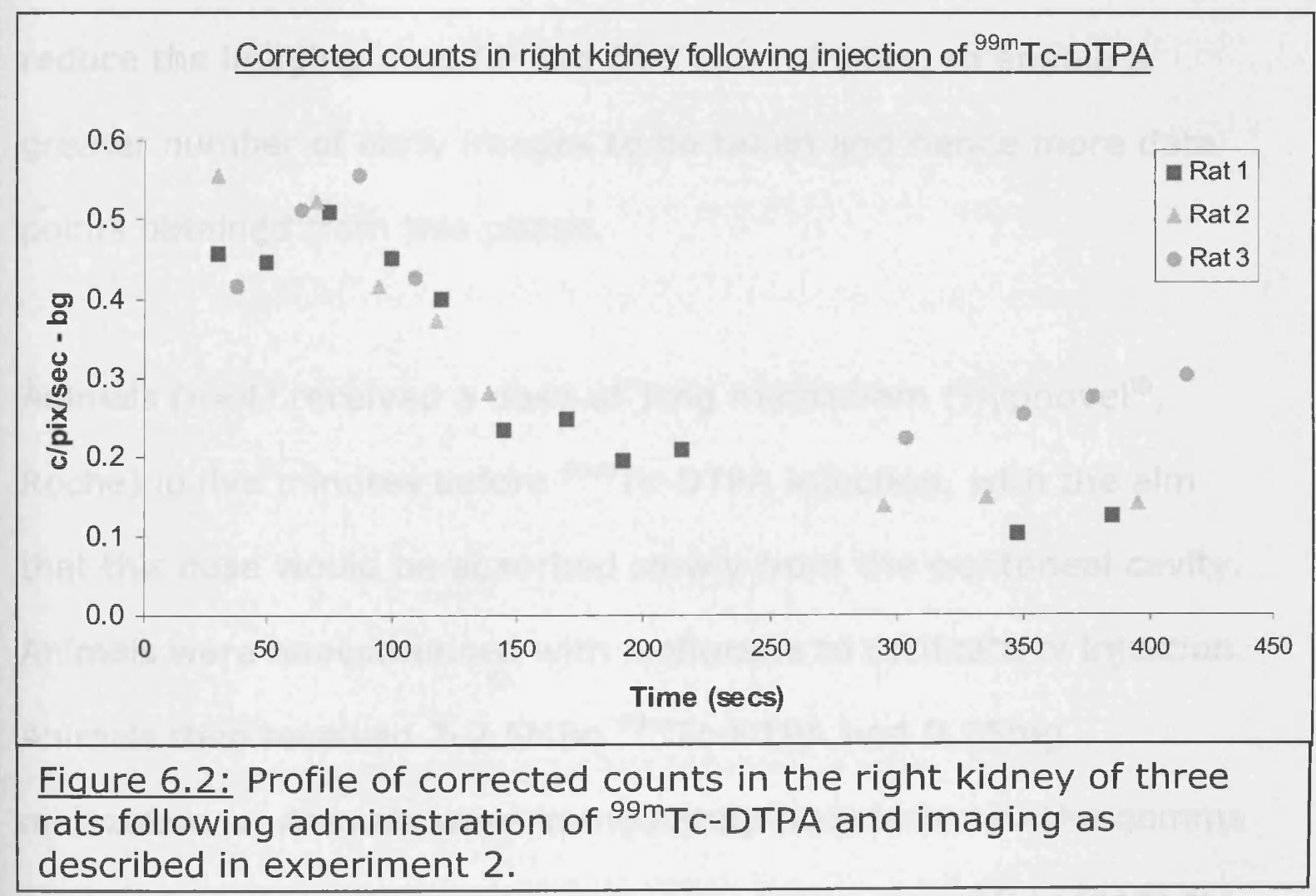




\section{Experiment 3}

It was apparent that longer-acting anaesthesia was required in order to be able to obtain sufficient early images. Petersen (1996) demonstrated that sedative doses of midazolam $(0.4 \mathrm{mg}$ followed by $1 \mathrm{mg} / \mathrm{hr}$ iv infusion) had no significant effect on GFR, as determined by inulin clearance, in rats. It was therefore decided to incorporate this anaesthetic into our regimen, with the aim of keeping the animals immobile on the camera for longer. It was also decided to reduce the imaging time for the first two minutes, to enable a greater number of early images to be taken and hence more data points obtained from this phase.

Animals $(n=4)$ received a dose of $1 \mathrm{mg}$ midazolam (Hypnove ${ }^{\circledR}$, Roche) ip five minutes before ${ }^{99 m}$ Tc-DTPA injection, with the aim that this dose would be absorbed slowly from the peritoneal cavity. Animals were anaesthetised with isoflurane to facilitate iv injection. Animals then received $2-2.5 \mathrm{MBq}{ }^{99 \mathrm{~m}} \mathrm{Tc}-\mathrm{DTPA}$ and $0.25 \mathrm{mg}$ midazolam iv. Animals were immediately transferred to the gamma camera and sequential posterior images taken over 10 seconds for the first two minutes, then over 20 seconds for the next two minutes, then over 30 seconds at $4,4.5$ and 7 minutes. ROIs were defined and processed as described previously. 
The new anaesthetic regimen used in this experiment was successful. Animals remained immobile on the camera, enabling a more comprehensive sequence of images to be obtained. They began to wake shortly after 7 minutes post-iv injection, so the sedation was ideally timed to fit in with our imaging protocol. It was decided to keep this regimen in place for use in future experiments. Figure 6.3 shows the corrected count profile obtained for the right kidney. It can be seen that the accumulation and elimination phases are clearly defined. Average time to peak was 119 seconds. Counts in rat 4 are lower as some of the dose was retained at the injection site. The counts obtained in the left kidney followed a similar profile with an average time to peak of 113 seconds. The imaging protocol used here enabled us to obtain enough data to characterise the accumulation phase of ${ }^{99 \mathrm{~m}} \mathrm{Tc}$-DTPA in the kidneys, and hence accurately characterise GFR. 


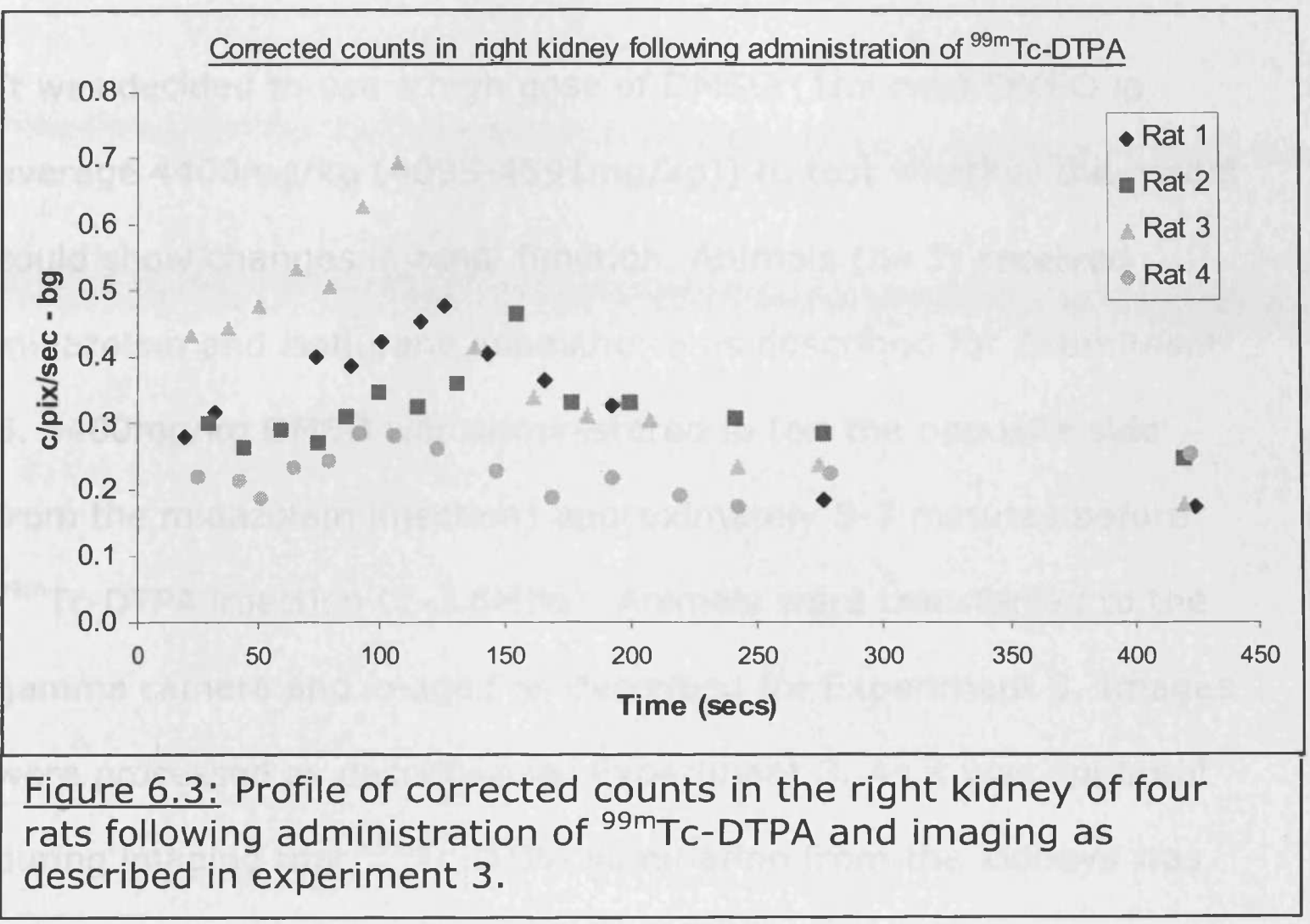




\section{Positive control}

It was decided to use a high dose of DMSO (1m/ neat DMSO ip, average $4400 \mathrm{mg} / \mathrm{kg}(4095-4691 \mathrm{mg} / \mathrm{kg}))$ to test whether the model could show changes in renal function. Animals $(n=3)$ received midazolam and isoflurane anaesthesia as described for Experiment 3. $4400 \mathrm{mg} / \mathrm{kg}$ DMSO was administered ip (on the opposite side from the midazolam injection) approximately 5-7 minutes before ${ }^{99 m}$ Tc-DTPA injection (2-2.5MBq). Animals were transferred to the gamma camera and imaged as described for Experiment 3. Images were processed as described for Experiment 3. As it was apparent during imaging that ${ }^{99 \mathrm{~m}} \mathrm{Tc}$-DTPA elimination from the kidneys was substantially reduced or abolished, additional 30-second images were taken until 10 minutes post- ${ }^{99 m}$ Tc-DTPA injection.

To estimate the duration of effect by DMSO, one rat received DMSO as described above and was injected with ${ }^{99 m}$ Tc-DTPA approximately 1 hour later (anaesthesia: as above, plus $0.25 \mathrm{mg}$ midazolam iv and isoflurane at time of ${ }^{99 \mathrm{~m}}$ Tc-DTPA injection), then imaged as described above.

Figure 6.4 shows the profile of counts obtained for the right kidney in the three rats who received ${ }^{99 \mathrm{~m}}$ Tc-DTPA 5 minutes after 
$4400 \mathrm{mg} / \mathrm{kg}$ ip DMSO. It can be seen that administration of DMSO has dramatically altered the shape of the plots. Accumulation in the kidneys is much slower, and no elimination phase is apparent during the imaging period. Lower counts were obtained for Rat 3, as some of the injected ${ }^{99 \mathrm{~m}}$ Tc-DTPA was retained at the injection site, but it is still clear that ${ }^{99 \mathrm{~m}}$ Tc-DTPA accumulated slowly in the kidneys with no elimination apparent. There was also no discernible accumulation in the bladder during the imaging period.

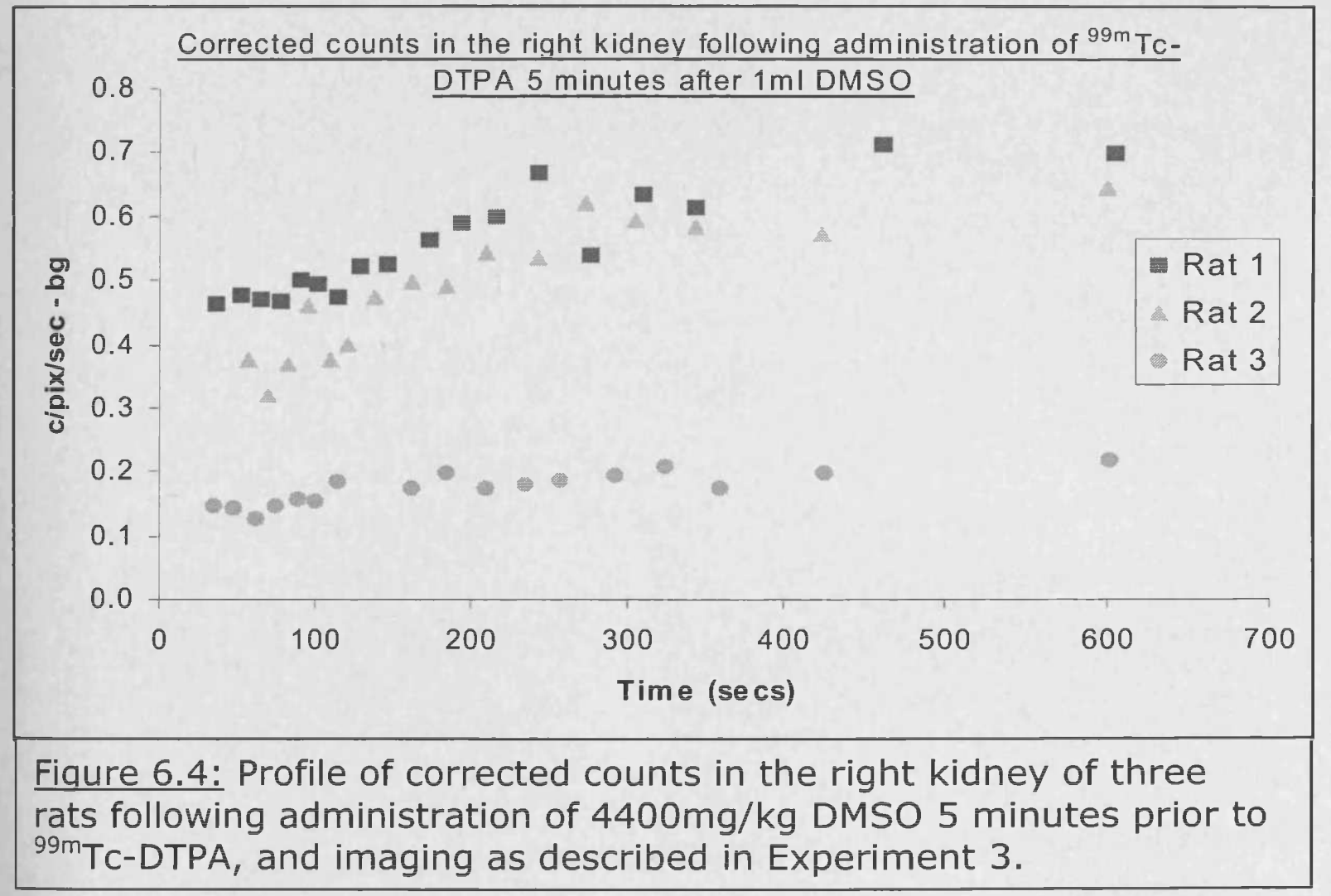

As can be seen in Figure 6.5, renal function was still compromised in the rat that received ${ }^{99 \mathrm{~m}}$ Tc-DTPA an hour after DMSO administration. The accumulation phase appeared to have a slightly shallower slope than in the control animals who had not received 
DMSO. The peak was also slightly later than in animals who had not received DMSO, occurring at 141 seconds in the right kidney and 212 seconds in the left kidney (compared to averages of 119 and 113 seconds in the control animals), and was flatter in shape. The slope of the elimination phase was also shallower, suggesting that elimination of ${ }^{99 m}$ Tc-DTPA from the kidneys was still compromised. However, some bladder accumulation of ${ }^{99 \mathrm{~m}} \mathrm{Tc}$-DTPA was apparent. Counts in this region increased after 141 seconds, corresponding to the time at which counts in the right kidney began to decline.

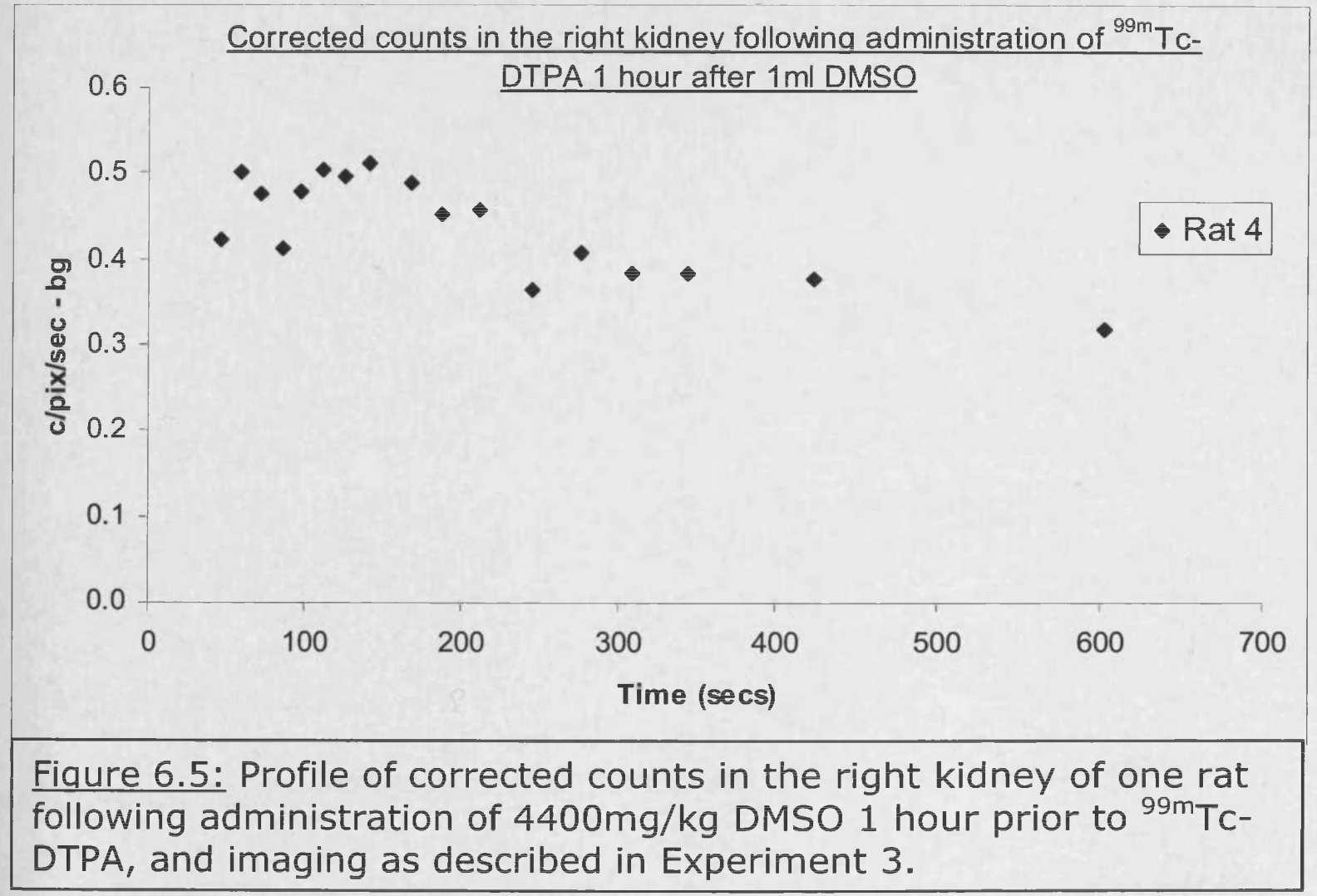


Examination of scintigraphic images from control and DMSO-treated rats also clearly shows the delay in ${ }^{99 m}$ Tc-DTPA renal elimination caused by DMSO (Figure 6.6).

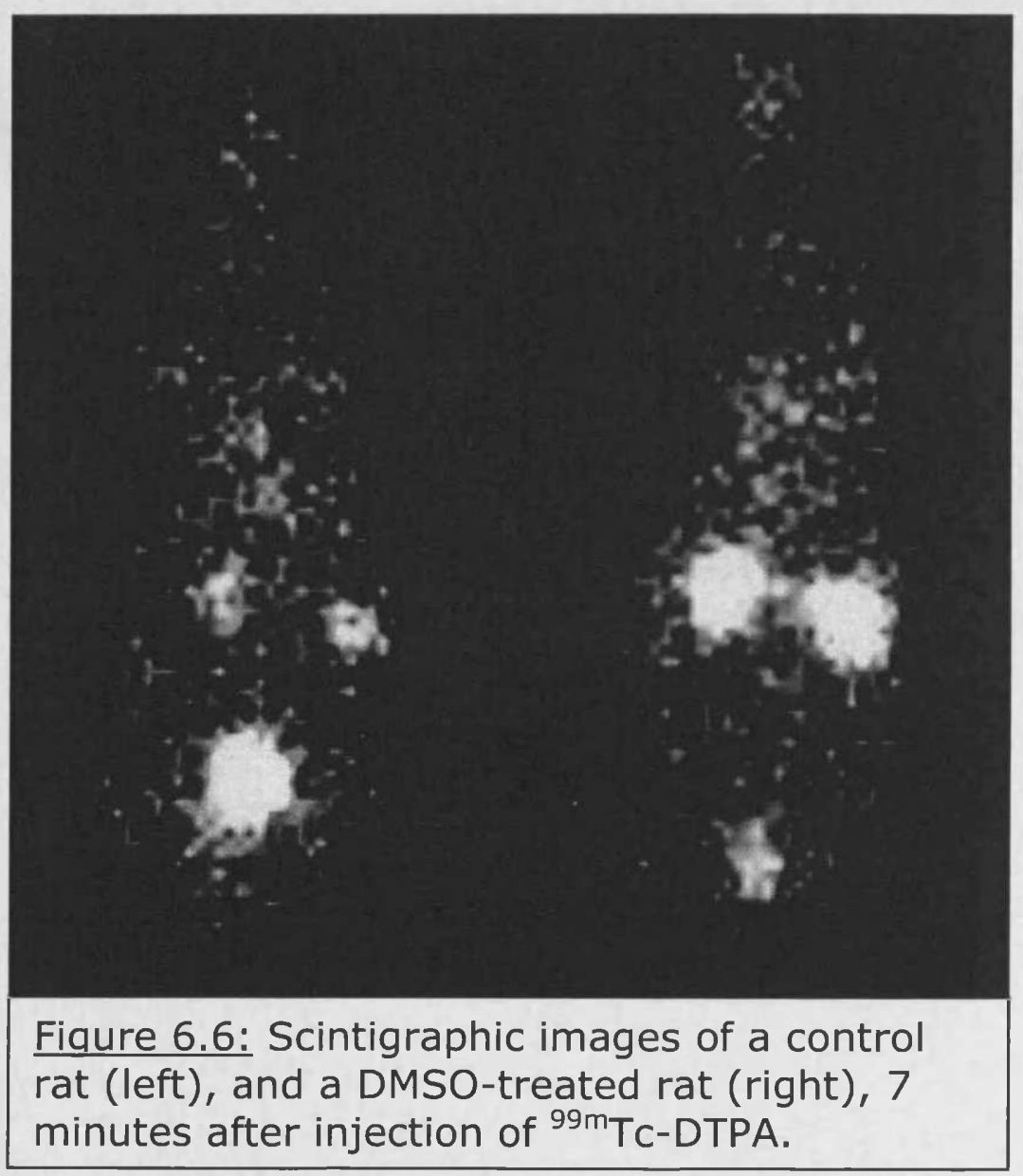




\subsubsection{Crossover-type method}

The method described above (Experiment 3 ) is sufficient to obtain enough information to accurately characterise the uptake and removal of ${ }^{99 \mathrm{~m}} \mathrm{Tc}$-DTPA from kidneys. The rapid elimination of ${ }^{99 \mathrm{~m}} \mathrm{Tc}$ DTPA makes this method suitable for crossover-type experiments, where each animal is used as its own control. It was decided to use this type of experimental design to test the effects of commonly used doses of pharmaceutical excipients on GFR.

A true crossover design for this experiment (ie. with some animals having the renogram in the presence of excipient taken before the control renogram) could prove problematic, as this would raise the question of how long the washout period should be between excipient administration and the control renogram. There is no firm basis in the literature to decide this. An experimental design where the control renogram is obtained first, followed by the renogram in the presence of the excipients, was therefore selected to avoid this issue.

Anaesthesia and imaging were as described in Experiment 3 above. Extra 30-second images were taken to ensure that the later stages of the count profile were well defined. A control ${ }^{99 m}$ Tc-DTPA 
renogram was taken and, following a break of at least 2 hours, animals were dosed with excipient and a further renogram performed. Doses of excipients used are shown in Table 6.1. One control renogram and one excipient renogram were carried out in each rat. The penile vein was used for intravenous dosing.

Table 6.1: Excipient doses used in crossover-type studies.

\begin{tabular}{|c|c|c|c|c|}
\hline Excipient & $\begin{array}{c}\text { Dose } \\
(\mathrm{mg} / \mathrm{kg})\end{array}$ & $\begin{array}{c}\text { Administered } \\
\text { as }\end{array}$ & Route & $\begin{array}{c}\text { Time before } \\
\text { DTPA }\end{array}$ \\
\hline DMSO & $393^{*}$ & $\begin{array}{l}20 \% \text { solution, } \\
0.5 \mathrm{ml}\end{array}$ & i.p. & $6-9$ mins \\
\hline PG & 829 & $\begin{array}{l}40 \% \text { solution, } \\
2 \mathrm{ml} / \mathrm{kg}\end{array}$ & i.p. & $6-9$ mins \\
\hline Solutol & 100 & $\begin{array}{l}12.5 \% \text { solution, } \\
0.8 \mathrm{ml} / \mathrm{kg}\end{array}$ & i.v. & $2-3$ mins \\
\hline Tween 80 & 85 & $\begin{array}{l}10 \% \text { solution, } \\
0.8 \mathrm{ml} / \mathrm{kg}\end{array}$ & i.v. & $2-3$ mins \\
\hline
\end{tabular}

$* 0.5 \mathrm{ml}$ of a $20 \%$ solution administered to each rat; corresponding to a dose of $384-402 \mathrm{mg} / \mathrm{kg}$. The mean dose, $393 \mathrm{mg} / \mathrm{kg}$, is used in the text.

\section{Data analysis}

Partial AUCs were calculated using WinNonLin (Pharsight), using a log-linear method.

To enable any dose loss during injection or retention at the injection site to be taken into account when comparing AUCs between 
renograms and between rats, an estimate of the actual dose rats received was calculated. This was done by drawing a ROI around the rat on the first image obtained after ${ }^{99 \mathrm{~m}} \mathrm{Tc}$-DTPA injection, and obtaining the number of counts in this ROI. Where injection site retention had occurred, this was accounted for by drawing a ROI around the site of retention and subtracting the number of counts in this ROI from the total number of counts in the rat.

Partial AUCs were corrected for dose by dividing by dose and multiplying by 100 to convert to a percentage. This is similar to the veterinary and clinical method of summing the total counts over an early time period (eg. 30-90 secs), and converting to percentage of dose to calculate GFR. Cumulative $A_{U} C_{0-420 \text { secs }}$ were also compared graphically between control and treatment renograms.

It was initially planned to perform pharmacokinetic analysis on the corrected count profiles in order to determine the rate constants and half-lives associated with the uptake and elimination phases, and enable these to be compared between control and treatment renograms. A two-compartment infusion model provided a good fit for some of the data, but would not fit all of the renograms and tended to yield very small values for either $\alpha$ or $\beta$, suggesting that one of the rate constants was redundant. However, a onecompartment infusion model did not fit the data well either; fit of 
the peak and elimination phase were very poor, even when elimination appeared to be monophasic. It was therefore decided not to use compartmental analysis, as it did not appear possible to find a model which would adequately fit all of the renograms to enable comparisons to be made.

Statistical significance was assessed using a one-way ANOVA, performed using SPSS (SPSS Inc.).

\subsection{Results}

\subsubsection{DMSO $393 \mathrm{mg} / \mathrm{kg}$}

The striking effects seen in the positive control experiment with $4400 \mathrm{mg} / \mathrm{kg}$ neat DMSO did not occur when the dose was reduced to $393 \mathrm{mg} / \mathrm{kg}$, a dose used in pre-clinical studies. Dose-corrected kidney $A \cup C_{30-90 s e c}$ was significantly increased by pre-treatment with this dose of DMSO (Table 6.2), but dose-corrected $A_{U C} C_{0-420 s e c}$ was unaffected. Dose-corrected kidney count profiles are shown in Figure 6.7.No differences were apparent between cumulative $A \cup C_{0-}$ 420 sec curves from control and treatment renograms. 
No results are reported for the test renogram from the left kidney of Rat $B$, as the counts in the kidney began to increase again after 301 seconds. This did not occur in the right kidney for this rat, suggesting that it is due to changes in the left kidney itself, rather than a whole body effect. Also, the right kidney in the test renogram of rat $\mathrm{C}$ could not be analysed, as a large ROI appeared which swamped the kidney and prevented it from being resolved. The cause of this is unclear, as the ip injection solutions were not found to be contaminated with ${ }^{99 \mathrm{~m}} \mathrm{Tc}$. It was attributed to an accumulation of ${ }^{99 \mathrm{~m}} \mathrm{Tc}$ in the peritoneal cavity, due to increased permeability of part of the membrane caused by the DMSO, or damage to the peritoneal membrane during ip injections. This occurrence was not unique to this rat; other instances where it occurred are described later. The right kidney of rat $D$ is not included in any of the analysis, as the renograms showed that the function of this kidney was impaired. This was demonstrated by the fact that the counts in this kidney increased with a very shallow slope, and no peak or elimination phase was apparent during the imaging period. This fits the description of a time-activity curve for a poorly functioning kidney given by Kerl and Cook (2005).

Rat $C$ awoke early in the control renogram, as did Rat $D$ in the test renogram, thus curtailing the imaging period. $A U C_{0-420 \text { secs }}$ for these rats therefore include a small amount of prediction. However, the 
kidney count profiles for both of these rats had already entered the final elimination phase, so $A \cup C_{0-420 s e c}$ is not likely to be in error by this extrapolation. Excluding the data from these rats from the statistical analysis of $\mathrm{AUC}_{0-420 \mathrm{sec}}$ does not alter the outcome.

Table 6.2: Effect of $393 \mathrm{mg} / \mathrm{kg}$ DMSO on parameters describing ${ }^{99 m}$ Tc-DTPA uptake and elimination from the kidney.

\begin{tabular}{|cc|cc|cc|}
\cline { 2 - 6 } \multicolumn{1}{l|}{} & \multicolumn{2}{|c|}{ AUC $_{\mathbf{3 0}-90 \mathrm{sec}}(\%)$} & \multicolumn{2}{c|}{ AUC 0-420sec (\%) } \\
\hline Rat A & R & 5.31 & 5.59 & 38.02 & 30.80 \\
& L & 3.48 & 4.75 & 34.16 & 25.16 \\
Rat B & R & 4.29 & 6.31 & 24.50 & 35.08 \\
& L & 4.51 & & 23.99 & \\
Rat C & R & 4.47 & & 21.71 & \\
& L & 4.25 & 4.68 & 25.07 & 28.37 \\
Rat D & L & 3.45 & 5.36 & 23.31 & 33.06 \\
\hline Mean & 4.25 & $5.34 *$ & 27.25 & 30.49 \\
( \pm SD) & $( \pm 0.64)$ & $( \pm 0.67)$ & $( \pm 6.23)$ & $( \pm 3.90)$ \\
\hline
\end{tabular}

$*=$ significantly different from control $p<0.05 . R, L=$ right or left kidney respectively 

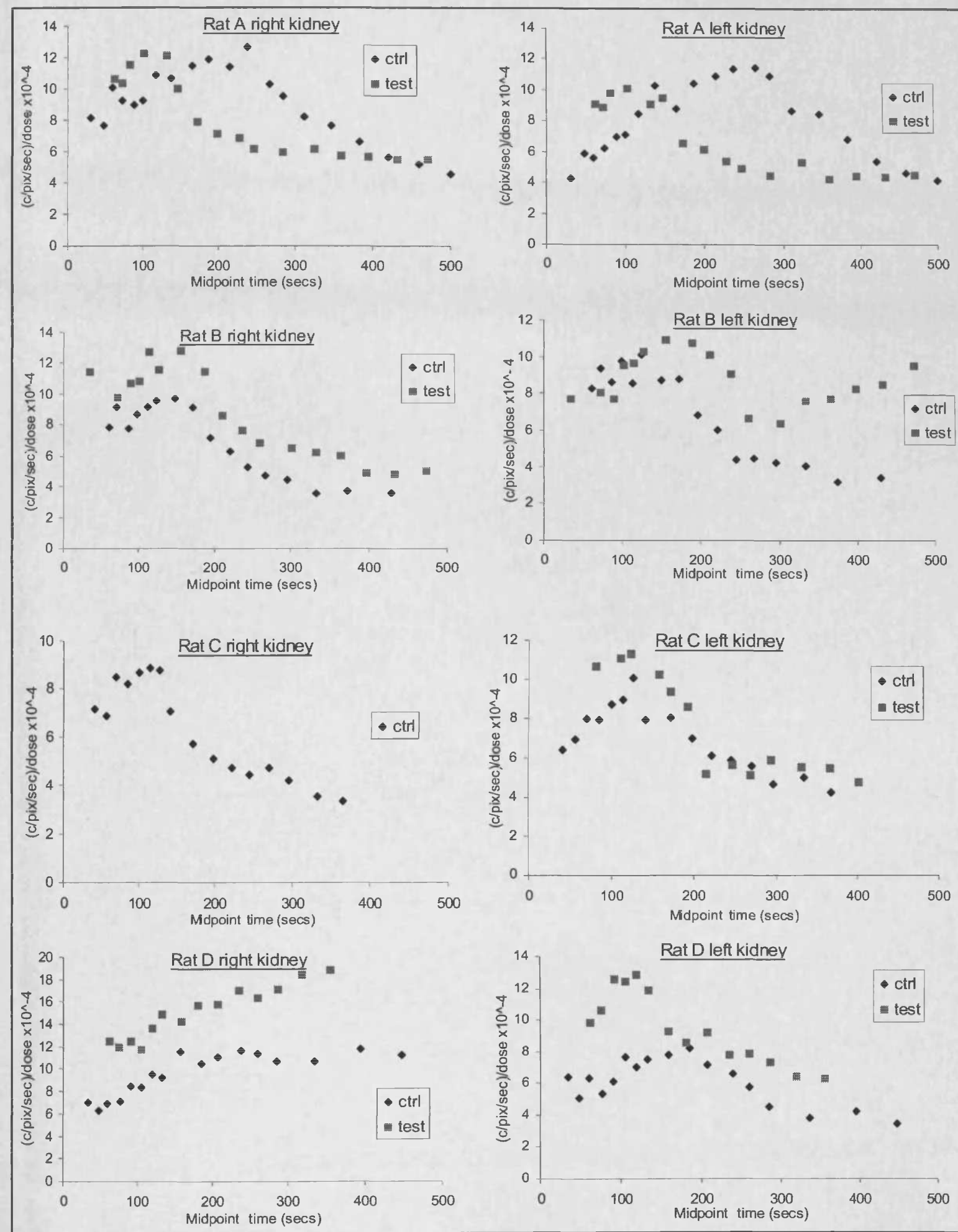

Fiqure 6.7: Effects of $393 \mathrm{mg} / \mathrm{kg}$ DMSO on dose-corrected count profile of ${ }^{99 \mathrm{~m}}$ Tc-DTPA in rat kidneys. 


\section{$6.3 .2829 \mathrm{mg} / \mathrm{kg} P G$}

A dose of $829 \mathrm{mg} / \mathrm{kg}$ PG had no significant effects on the dosecorrected kidney AUCs (Table 6.3). There were no discernible effects on the cumulative $A_{U} C_{0-420 s e c}$ curves. Kidney count profiles are shown in Figure 6.8.

Table 6.3: Effect of $829 \mathrm{mg} / \mathrm{kg}$ PG on parameters describing ${ }^{99 \mathrm{~m} T \mathrm{~T}-}$ DTPA uptake and elimination from the kidney.

\begin{tabular}{|c|c|c|c|c|}
\hline & \multicolumn{2}{|c|}{ AUC $_{30-90 \sec }(\%)$} & \multicolumn{2}{|c|}{ AUC $_{0-420 \sec }(\%)$} \\
\hline & Control & Test & Control & Test \\
\hline \multirow[t]{2}{*}{ Rat E } & 5.45 & 6.57 & 27.26 & 37.81 \\
\hline & 5.67 & 5.82 & 27.45 & 38.86 \\
\hline \multirow[t]{2}{*}{ Rat F } & 4.85 & 5.56 & 29.27 & 35.01 \\
\hline & 5.72 & 4.19 & 35.44 & 31.73 \\
\hline \multirow[t]{2}{*}{ Rat G } & 5.38 & 6.37 & 31.76 & 31.14 \\
\hline & 4.73 & & 29.16 & \\
\hline Rat H L & 4.79 & 4.03 & 32.53 & 24.34 \\
\hline Mean & 5.23 & 5.42 & 30.41 & 33.15 \\
\hline$( \pm$ SD) & $( \pm 0.43)$ & $( \pm 1.08)$ & $( \pm 2.98)$ & $( \pm 5.32)$ \\
\hline
\end{tabular}

$\mathrm{R}, \mathrm{L}=$ right or left kidney respectively.

No values for the right kidney of rat $\mathrm{H}$ are included in the table. In the control renogram, counts in this kidney started to rise again after 195 seconds, soon after the peak. This did not occur in the left kidney, and so appears to be due to events in this particular kidney rather than a whole body effect. The slope of the accumulation phase is comparable to the other renograms, suggesting that this 
was not due to renal impairment. In the test renograms for the right kidney of rat $\mathrm{H}$ and the left kidney of rat $\mathrm{G}$, there was a ROI swamping the kidney on the scintigraphic images, which prevented this kidney from being resolved (as described for rat $C$ in the DMSO group). In the control renogram for rat $\mathrm{H}$, imaging stopped at 280 seconds, as the rat awoke at this point, preventing further imaging. This means that the $A U C_{0-420 s e c}$ calculations for this rat include 140 seconds of prediction. Kidney counts had entered the elimination phase at this point, but had not entered the terminal phase. As $\mathrm{AUC}_{0-420 \mathrm{sec}}$ was predicted from the slope of the last elimination phase, this could cause the parameter to be underestimated. A case could therefore be made for excluding this data from the statistical analysis; however, this value does not appear to be an outlier, and no significant effect of PG treatment was apparent even after this data was removed from the analysis for this parameter. 


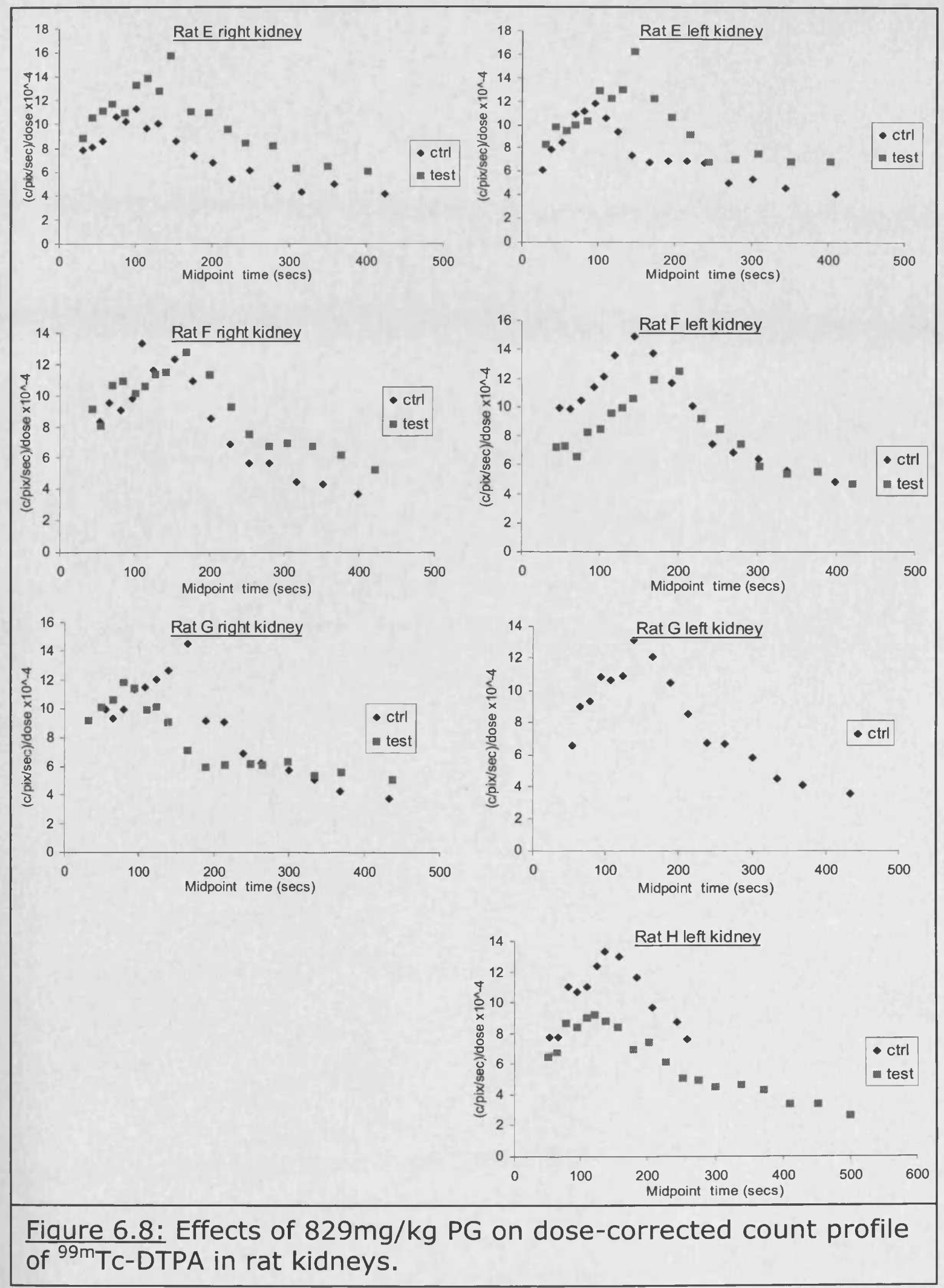




\section{$6.3 .3100 \mathrm{mg} / \mathrm{kg}$ Solutol HS15}

Pre-treatment with Solutol HS15 affected renal elimination of ${ }^{99 \mathrm{~m}} \mathrm{Tc}$ DTPA in two of the four rats in this treatment group (rats I and J). Dose-corrected $A \cup C_{0-420 s e c}$ was increased by $25 \%$ (Table 6.4 ). There were no significant changes in dose-corrected $A_{U} C_{30-90 s e c}$. Kidney count profiles are shown in Figure 6.10.

The cumulative $A \cup C_{0-420 s e c}$ curves in these rats were shifted to the right (Figure 6.9). This is likely to be due to the fact that the slope of the elimination phase in these rats became shallower after Solutol treatment (Figure 6.10), and dose-corrected $\mathrm{AUC}_{0-420 \mathrm{sec}}$ was increased. This means that corrected kidney counts at the later timepoints were higher, and made a greater percentage contribution to $\mathrm{AUC}_{0-420 \mathrm{sec}}$ than in the control renogram, thus causing the curves to shift to the right.

Difficulty in injecting rat $I$ in the Solutol group meant that there were two minor deviations from the experimental protocol in the test renogram for this rat. Solutol was injected 5 minutes before ${ }^{99 m}$ Tc-DTPA (rather than 2-3 minutes as in the other rats), and the ${ }^{99 m}$ Tc-DTPA was injected over a longer period ( 1 minute rather than 20-30 seconds in the other rats). This was corrected for by taking the midpoint of the injection time as time zero. 
Table 6.4: Effect of Solutol HS15 on parameters describing ${ }^{99 \mathrm{~m}} \mathrm{Tc}-$ DTPA uptake and elimination from the kidney.

\begin{tabular}{|c|c|c|c|c|c|}
\hline & \multicolumn{2}{|c|}{$A^{\prime} C_{30-90 \sec }(\%)$} & \multicolumn{2}{|c|}{$A U C_{0-420 \sec }(\%)$} \\
\hline & & Control & Test & Control & Test \\
\hline \multirow[t]{2}{*}{ Rat I } & $\overline{\mathbf{R}}$ & 5.27 & 6.10 & 27.58 & 41.29 \\
\hline & $\mathbf{L}$ & 3.94 & 4.78 & 21.97 & 36.54 \\
\hline \multirow[t]{2}{*}{ Rat J } & $\mathbf{R}$ & 5.41 & 5.36 & 26.45 & 39.62 \\
\hline & $\mathbf{L}$ & 5.09 & 4.18 & 26.73 & 35.17 \\
\hline \multirow[t]{2}{*}{ Rat $K$} & $\mathbf{R}$ & 4.62 & 5.86 & 33.14 & 32.91 \\
\hline & $\mathbf{L}$ & 3.69 & 5.98 & 33.67 & 35.84 \\
\hline \multirow[t]{2}{*}{ Rat L } & $\mathbf{R}$ & 4.22 & 4.37 & 24.89 & 25.95 \\
\hline & $\mathbf{L}$ & 4.02 & 4.46 & 23.55 & 26.19 \\
\hline \multirow{2}{*}{\multicolumn{2}{|c|}{$\begin{array}{l}\text { Mean } \\
( \pm \text { SD })\end{array}$}} & 4.53 & 5.14 & 27.25 & $34.19 *$ \\
\hline & & $( \pm 0.66)$ & $( \pm 0.78)$ & $( \pm 4.21)$ & $( \pm 5.64)$ \\
\hline
\end{tabular}

$*=$ significantly different from control $p<0.05 . R, L=$ right or left kidney respectively

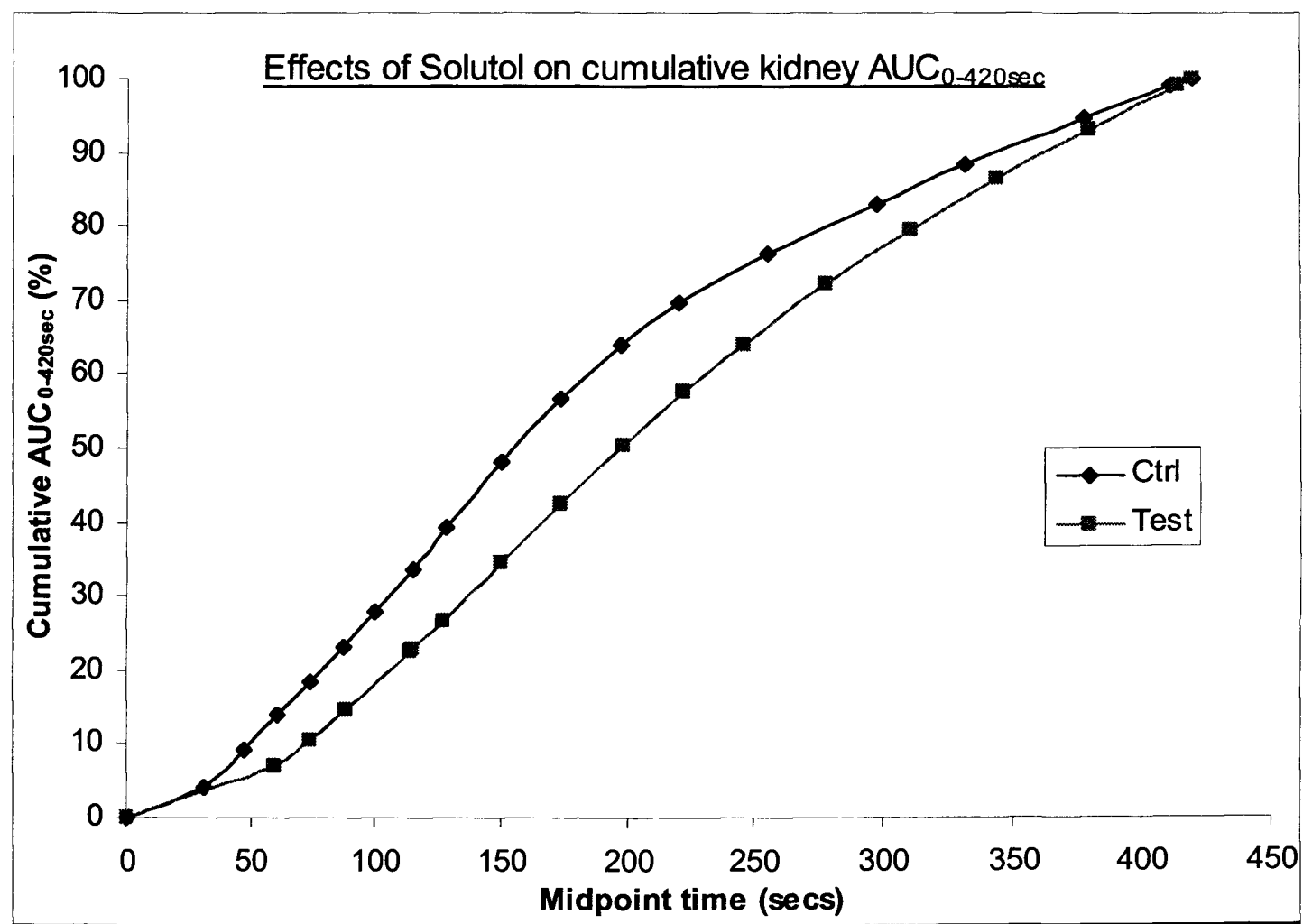

Figure 6.9: Effects of Solutol HS15 on cumulative ${ }^{99 m}$ Tc-DTPA AUC ${ }_{0-420}$ in right kidney of rat $\mathrm{B}$. 

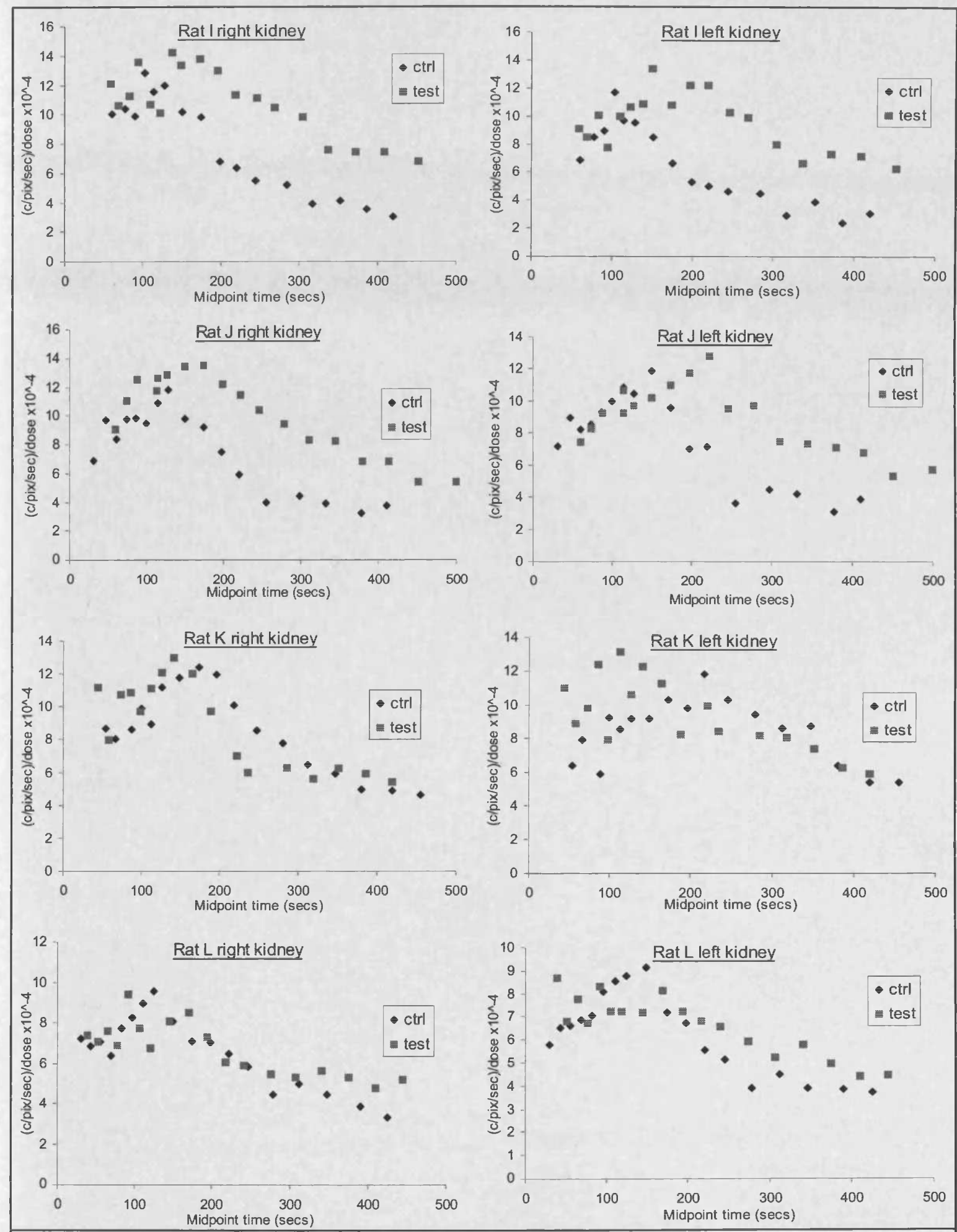

Fiqure 6.10: Effects of $100 \mathrm{mg} / \mathrm{kg}$ Solutol HS15 on dose-corrected count profile of ${ }^{99 \mathrm{~m}} \mathrm{Tc}$-DTPA in rat kidneys. 


\section{$\underline{6.3 .485 \mathrm{mg} / \mathrm{kg} \text { Tween } 80}$}

Pre-treatment with Tween $80(85 \mathrm{mg} / \mathrm{kg})$ caused a significant overall increase of $27 \%$ in dose-corrected kidney $\mathrm{AUC}_{0-420 \mathrm{sec}}$ (Table 6.5), due to changes in rats $\mathrm{M}, \mathrm{N}$ and $\mathrm{P}$ after excipient treatment. Kidney count profiles are shown in Figure 6.11. A small amount of the Tween dose was lost in rat $\mathrm{N}$, due to difficulty injecting.

However, unlike in the Solutol group, there were no apparent differences between the cumulative $A U C_{0-420 s e c}$ curves for control and test renograms. This may be due to the fact that the change in slope of the elimination phase and increase in $\mathrm{AUC}_{0-420 \mathrm{sec}}$ were more pronounced in the Solutol treated rats. This suggests that the partial AUCs are a more sensitive measure of changes in renal elimination than the cumulative $\mathrm{AUC}_{0-420 \mathrm{sec}}$ curves, as the former method was able to demonstrate an effect of both excipients on renal elimination. 
Table 6.5: Effect of Tween 80 on parameters describing ${ }^{99 \mathrm{~m}}$ Tc-DTPA uptake and elimination from the kidney.

\begin{tabular}{|c|c|c|c|c|}
\hline & \multicolumn{2}{|c|}{ AUC $_{30-90 \sec }(\%)$} & \multicolumn{2}{|c|}{ AUC $_{0-420 \sec }(\%)$} \\
\hline & Control & Test & Control & Test \\
\hline \multirow[t]{2}{*}{ Rat M } & 4.98 & 6.22 & 24.18 & 35.35 \\
\hline & 4.61 & 4.98 & 25.36 & 32.23 \\
\hline \multirow[t]{2}{*}{ Rat $\mathbf{N}$} & 4.93 & & 22.89 & \\
\hline & 4.47 & 5.14 & 22.66 & 30.34 \\
\hline \multirow[t]{2}{*}{ Rat $\mathbf{O}$} & 4.69 & 4.89 & 26.16 & 25.68 \\
\hline & 3.62 & 4.36 & 24.24 & 26.47 \\
\hline \multirow[t]{2}{*}{ Rat P } & 5.44 & 7.08 & 26.87 & 36.65 \\
\hline & 5.44 & 5.82 & 27.44 & 36.00 \\
\hline \multirow{2}{*}{$\begin{array}{l}\text { Mean } \\
( \pm \text { SD })\end{array}$} & 4.77 & 5.50 & 24.98 & $31.82^{*}$ \\
\hline & $( \pm 0.59)$ & $( \pm 0.93)$ & $( \pm 1.78)$ & $( \pm 4.51)$ \\
\hline
\end{tabular}

No parameters could be calculated for the right kidney of rat $\mathrm{N}$ after Tween treatment, as a large ROI appeared on the scintigraphic images which swamped the kidney, as described above. In the control renogram for rat $\mathrm{N}$, the rat awoke after 239 seconds, thus preventing further imaging. The $A \cup C_{0-420 s e c}$ calculations for this rat therefore include 181 seconds of prediction. A case could therefore be made for excluding this data. However, this value does not appear to be an outlier, and the increase in $A U C_{0-420 s e c}$ seen for the group remains significant when this rat's data are excluded from the analysis. 

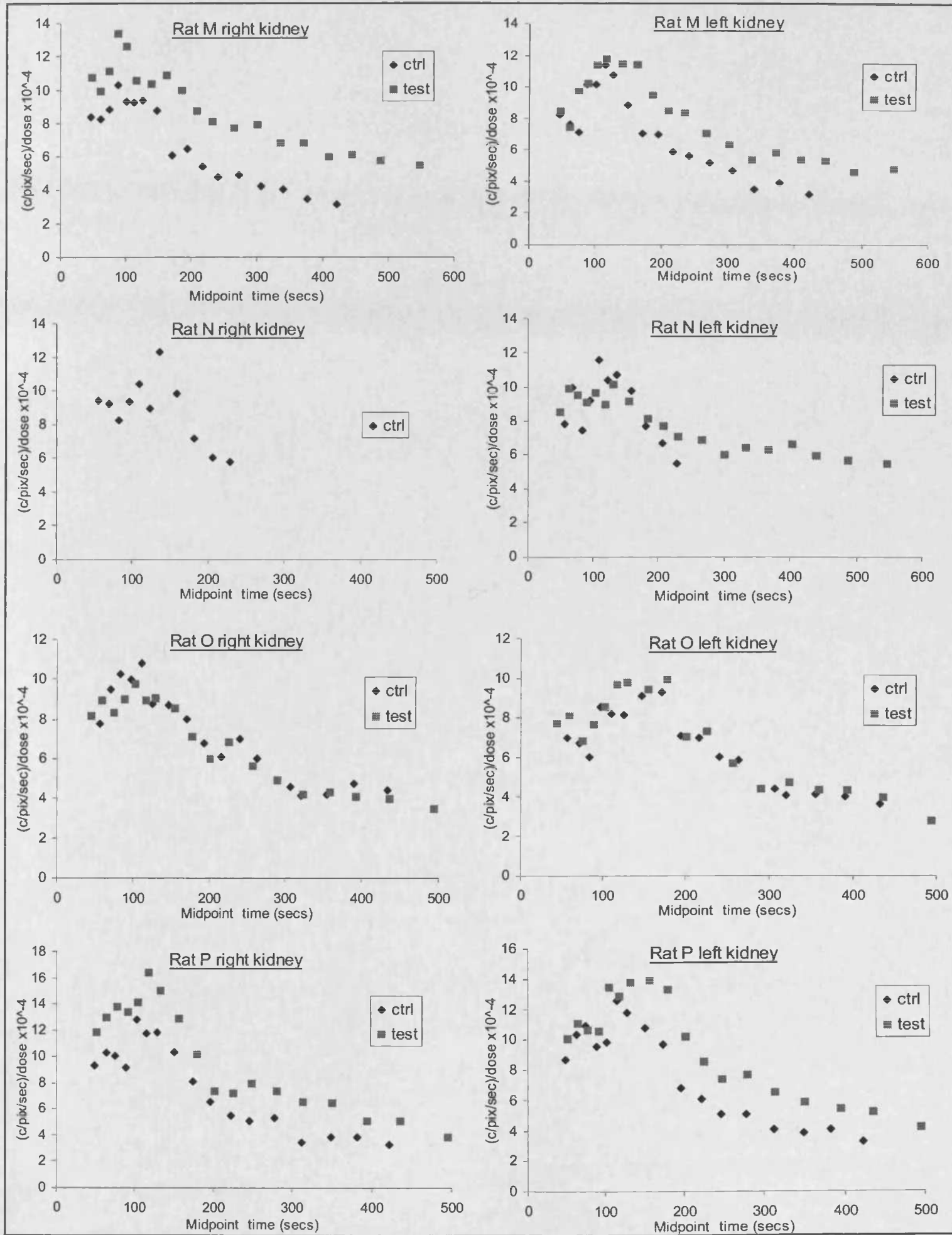

Fiqure 6.11: Effects of $85 \mathrm{mg} / \mathrm{kg}$ Tween 80 on dose-corrected count profile of ${ }^{99 \mathrm{~m}}$ Tc-DTPA in rat kidneys. 


\subsubsection{Positive control $-4400 \mathrm{mg} / \mathrm{kg}$ DMSO}

For comparative purposes, dose-corrected kidney AUCs were also calculated for the positive control experiment (Table 6.6). $\mathrm{AUC}_{30}$ $90 \mathrm{sec}$ was increased by $21 \%$ compared to the average control value, and $A \cup C_{0-420 \text { sec }}$ by $84 \%$. As no control renograms were carried out in these rats, no statistical analysis of these results was performed; however, it is worth noting that the $21 \%$ increase in $30-90$ sec was within the standard deviation for this parameter.

A direct comparison of cumulative $\mathrm{AUC}_{0-420 \mathrm{sec}}$ curves cannot be carried out as no control renograms were carried out in these animals; however, it appears that the curves for this group were flatter in shape than those from control animals in the other groups, and were generally shifted to the right. 
Table 6.6: Effect of $4400 \mathrm{mg} / \mathrm{kg}$ DMSO on parameters describing ${ }^{99 m}$ Tc-DTPA uptake and elimination from the kidney.

\begin{tabular}{|c|c|c|}
\hline & $\begin{array}{c}\text { AUC }_{30-90 s e c} \\
(\%)\end{array}$ & $\begin{array}{c}\text { AUC }_{0-420 s e c} \\
(\%)\end{array}$ \\
\hline $\begin{array}{ll}\text { Rat } 1 & R\end{array}$ & 6.41 & 52.74 \\
\hline $\mathbf{L}$ & 4.82 & 40.58 \\
\hline Rat $2 \mathbf{R}$ & 4.35 & 45.74 \\
\hline $\mathbf{L}$ & 4.67 & 46.09 \\
\hline Rat $3 \mathbf{R}$ & 7.39 & 62.74 \\
\hline $\mathbf{L}$ & 6.36 & 53.62 \\
\hline $\begin{array}{l}\text { Mean } \\
( \pm \text { SD })\end{array}$ & $\begin{array}{c}5.67 \\
( \pm 1.22)\end{array}$ & $\begin{array}{c}50.25 \\
( \pm 7.81)\end{array}$ \\
\hline
\end{tabular}

$\mathrm{R}, \mathrm{L}=$ right or left kidney respectively.

\subsubsection{Comparison of data from control renograms}

Table 6.7 shows the mean parameters describing ${ }^{99 m}$ Tc-DTPA uptake and elimination from the kidney for control renograms from all treatment groups. Standard deviations for the partial kidney AUCs from control renograms were $14 \%$ for $A U_{30-90 s e c}$ and $16 \%$

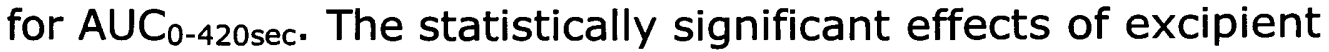
treatment reported above are unlikely to be due to variability between rats/renograms, as they are of a larger magnitude than this. 
Table 6.7: Comparison of parameters describing ${ }^{99 \mathrm{~m}}$ Tc-DTPA uptake and elimination from the kidney for control renograms from all treatment groups.

\begin{tabular}{|c|c|c|}
\hline Group & $\begin{array}{c}\text { AUC }_{30-90 s e c} \text { (\%) } \\
\text { (Mean } \pm \text { SD) }\end{array}$ & $\begin{array}{c}\text { AUC } \mathbf{O}_{\mathbf{4} 20 s e c} \text { (\%) } \\
(\text { Mean } \pm \text { SD) }\end{array}$ \\
\hline Rats A-D & $4.25( \pm 0.64)$ & $27.25( \pm 6.23)$ \\
Rats E-H & $5.23( \pm 0.43)$ & $30.41( \pm 2.98)$ \\
Rats I-L & $4.53( \pm 0.66)$ & $27.25( \pm 4.21)$ \\
Rats M-P & $4.77( \pm 0.59)$ & $24.98( \pm 1.78)$ \\
\hline Overall & $4.69( \pm 0.66)$ & $27.38( \pm 4.33)$ \\
\hline
\end{tabular}

\subsection{Discussion}

During this series of experiments a scintigraphic method has been successfully developed which enables us to investigate the effects of pharmaceutical excipients on the renal elimination of ${ }^{99 m}$ Tc-DTPA in rats. The combination of corrected count-time profiles and noncompartmental analysis enables better characterisation of the effects of the excipients than simply collecting urine at a single timepoint, as in other studies (Donadio et al., 1998), or summing uptake in early images (as in human studies, and the established veterinary method). The scintigraphic method developed here also has the advantage of being relatively non-invasive, compared to methods where repeated blood sampling is required. Although this method does not enable the calculation of an exact value for GFR, 
this is not necessary to perform comparisons between control and treatment groups. Additionally, validation against other methods would be required in order to do this with any certainty.

The substantial effects of $4400 \mathrm{mg} / \mathrm{kg}$ DMSO on the renal elimination of ${ }^{99 \mathrm{~m}} \mathrm{Tc}$-DTPA, seen in the positive control experiment, demonstrate that this dose of DMSO has the potential to substantially reduce or delay renal elimination of co-administered drugs. This has the potential to significantly affect their pharmacokinetics. The effects seen with this dose of DMSO also demonstrate that the method can detect changes in ${ }^{99 m}$ Tc-DTPA uptake into and elimination from the kidneys, and that these changes are reflected in our data analysis. Such a high dose of DMSO is unlikely to be used in pre-clinical studies. However, Watkins and Sherman (1992) used $2 \mathrm{ml} / \mathrm{kg}$ of DMSO (equivalent to $2200 \mathrm{mg} / \mathrm{kg}$ ) as a vehicle for digoxin in a pharmacokinetic study, demonstrating that high doses of this excipient are sometimes used as injection vehicles.

There are several potential explanations for the effects of this dose of DMSO. The kidney count profiles fit the description given by Kerl and Cook (2005) of those seen for poorly functioning kidneys, with counts in the kidney increasing with a very shallow slope, and no peak or elimination phase apparent. The excipient may therefore have had a toxic effect on the kidney, causing damage which 
impaired renal function. It is also possible that DMSO reduced renal blood flow. Kassell et al. (1983) reported that an iv dose of $6 \mathrm{~g} / \mathrm{kg}$ DMSO increased cerebral blood flow and reduced renal blood flow in dogs. This in turn would be expected to reduce GFR, and could be responsible for the changes described above, although the dose of DMSO used here $(4400 \mathrm{mg} / \mathrm{kg})$ is smaller. DMSO has been reported to cause osmotic diuresis in humans. Gunn and Acomb (1986) reported an increase in plasma osmolality following DMSO treatment for post-operative hemiplegia in humans, accompanied by diuresis. They noted that $50 \%$ of the dosed DMSO was excreted in the urine. Muther and Bennett (1980) also reported osmotic diuresis after DMSO administration to humans, at a dose of $1 \mathrm{~g} / \mathrm{kg}$ for 3 days. There were no changes in serum $\mathrm{Cr}$ or $\mathrm{CL}_{\mathrm{Cr}}$. However, diuresis should result in faster elimination of ${ }^{99 \mathrm{~m}} \mathrm{Tc}-\mathrm{DTPA}$, not the delayed elimination seen in our experiment. Ali and Mousa (2001) dosed Wistar rats with $0.25-1 \mathrm{ml} / \mathrm{kg}(275-1100 \mathrm{mg} / \mathrm{kg})$ DMSO ip for 10 days. They reported that the excipient did not significantly affect plasma creatinine levels, measured 24 hours after the last dose of DMSO. This suggests that the effects of DMSO are transient even after repeated dosing. Unfortunately they do not report any renal function parameters shortly after DMSO was administered, for comparison with the results of the present study. 
The significant $26 \%$ increase in kidney $A_{U} C_{30-90 s e c}$ caused by the lower dose of $393 \mathrm{mg} / \mathrm{kg}$ DMSO indicates that ${ }^{99 \mathrm{~m}} \mathrm{Tc}$-DTPA uptake into the kidneys was faster after DMSO treatment. This suggests an increase in GFR, as ${ }^{99 m}$ TC-DTPA only enters the kidney through glomerular filtration and is not subject to any active uptake processes (Kerl and Cook, 2005). The fact that there was no effect on $\mathrm{AUC}_{0-420 \mathrm{sec}}$ suggests that elimination from the kidney itself was not significantly affected.

DMSO is commonly used in pre-clinical studies at doses of $10-20 \%$ $2 \mathrm{ml} / \mathrm{kg}$, equivalent to $220-440 \mathrm{mg} / \mathrm{kg}$ (Marcel DeMatas, personal communication). We have therefore shown an effect of DMSO on renal elimination at a dose which is used in pre-clinical studies. This dose of DMSO has the potential to affect renal elimination of coadministered drugs by increasing GFR, and hence affect their pharmacokinetics. The extent to which a drugs pharmacokinetics are affected will depend on its disposition characteristics. Increasing GFR could increase the rate of renal elimination of the drug, hence reducing plasma $t_{1 / 2}$. Drugs which also undergo metabolism could show a shift in the proportion of drug cleared renally and hepatically, with an increase in $A_{e}$ and reduction in metabolism. Drugs which only undergo a small amount of renal elimination, or whose rate of renal elimination is determined by active transport 
rather than glomerular filtration, are less likely to be affected by an increase in GFR.

It is of interest that the effect seen at this low dose of DMSO is different from that seen at $4400 \mathrm{mg} / \mathrm{kg}$, rather than the same effect being seen to a lesser extent. The reported effects of DMSO which may impact upon renal elimination are described above. It may be that different mechanisms are responsible for the effects seen at the different doses. The effects seen after $393 \mathrm{mg} / / \mathrm{kg}$ DMSO do not appear to be explained by any of the reported renal effects of DMSO described above. A reduction in kidney blood flow would result in a reduction rather than an increase in GFR. The osmotic diuresis described by Gunn and Acomb (1986), and Muther and Bennett (1980), is likely to be mediated by changes in water reabsorption in the kidney rather than an increase in GFR.

PG, at a dose of $829 \mathrm{mg} / \mathrm{kg}$, does not appear to significantly affect the renal elimination of ${ }^{99 m}$ Tc-DTPA, and so is not likely to affect renal elimination of drugs by altering GFR. There are reports in the literature where PG has affected renal function. The excipient has been reported to cause nephrotoxicity in some patients (Hayman et al., 2003; Yaucher et al., 2003). Yaucher et al. (2003) reported that 8 out of 128 patients receiving iv infusions of lorazepam developed renal toxicity due to PG in the injection vehicle, demonstrated by 
elevated serum creatinine. The levels of PG and duration of dosing at which this effect occurred were variable; toxicity occurred after 3-60 days treatment with lorazepam/PG (median 9 days), at PG levels of $186-3450 \mu \mathrm{g} / \mathrm{ml}$ (mean $1103 \mu \mathrm{g} / \mathrm{ml}$ ). There are additional case reports of PG-mediated nephrotoxicity occurring in patients who had received intravenous doses of drugs formulated in PG (Hayman et al., 2003). The dose of PG used in the present study would give theoretical maximum blood PG levels of $1.30 \mathrm{mg} / \mathrm{ml}$, assuming distribution into total body water. This is within the range where renal effects of PG occurred in Yaucher et al. The patients in Yaucher et al.'s study were intensive care and burns unit patients, and so may be more succeptible to the effects of PG, as they were seriously ill and hence under greater physical stress than healthy individuals. Additionally, the effect only occurred in $6 \%$ of patients in the study, and after varying PG levels and durations of dosing. This demonstrates that the renal effects of PG are not predictable, and may not occur in all individuals. The fact that our subjects received PG by ip bolus rather than iv infusion (giving sustained plasma levels of PG) may be another reason why no effect was seen in our study. However, an in vitro study by Morshed et al. (1994) reported that the toxic effects of PG on human proximal tubule cells were evident after just 10 minutes of exposure to $2 \% \mathrm{PG}$, as demonstrated by increased lactate release. Membrane damage was evident after 15 minutes exposure (as shown by ${ }^{51} \mathrm{Cr}$ 
release). This suggests that toxicity could occur after the short exposure given by a bolus dose of PG, as well as after the sustained exposure given by an infusion of the excipient.

PG is used in pre-clinical studies at doses of up to $50 \% 2 \mathrm{ml} / \mathrm{kg}$ $(1036 \mathrm{mg} / \mathrm{kg})$, although lower levels are usually used (Marcel DeMatas, personal communication). The results of the present study show that PG does not affect renal elimination of ${ }^{99 \mathrm{~m}}$ Tc-DTPA at a dose of $829 \mathrm{mg} / \mathrm{kg}$, suggesting that renal effects of this excipient are unlikely to be seen at doses commonly used in pre-clinical studies.

The increase in dose-corrected kidney $\mathrm{AUC}_{0-420 \text { sec }}$ caused by $100 \mathrm{mg} / \mathrm{kg}$ Solutol HS15 suggests that this excipient delayed ${ }^{99 \mathrm{~m}} \mathrm{Tc}-$ DTPA elimination from the kidney. The lack of effect on $\mathrm{AUC}_{30-90 \mathrm{sec}}$ suggests that ${ }^{99 \mathrm{~m}}$ Tc-DTPA uptake into the kidney (ie. GFR) was not affected. The fact that Solutol treatment only affected ${ }^{99 \mathrm{~m}}$ Tc-DTPA elimination from the kidneys in two of the four rats is of interest, as no reason was apparent for this during the experiment. It therefore appears that the rats may have differed in their susceptibility to the effects of Solutol. The group size is too small to estimate the true incidence of this effect. This could cause problems in early pharmacokinetic studies where this dose of Solutol is used, as the effects of this excipient on renal elimination would not be predictable. 
In pre-clinical studies, Solutol is often used at a dose of $300 \mathrm{mg} / \mathrm{kg}$ (Marcel DeMatas, personal communication). The dose used in the present studies is one-third of this. A lower dose was used in the present study to enable comparison with the work of Bittner et al. (2003b) described in the introduction, which used a Solutol dose of $5 \% 2 \mathrm{ml} / \mathrm{kg}(100 \mathrm{mg} / \mathrm{kg})$. It is possible that an increase in the magnitude of the renal effects of Solutol would be seen at higher doses of the excipient.

As Solutol appeared to delay ${ }^{99 \mathrm{~m}}$ Tc-DTPA elimination from the kidney itself, it has the potential to affect the pharmacokinetics of drugs which undergo active reabsorption from the kidney. The amount of time these drugs spend in the kidney would be increased by the effects of Solutol, thus increasing the length of time they are available for reabsorption. If the reabsorption of these drugs from the kidneys is increased, this could mean an increase in plasma concentrations. However, for drugs which are not solely eliminated by the kidney, elimination by other routes (such as metabolism) may increase to compensate for any increase in reabsorption from the kidneys. Pharmacokinetic studies using such drugs would be necessary to determine whether this was the case, and would be hampered by the fact that the effect was not seen in all rats. However, even if there is no effect on drug plasma levels, it would 
still be desirable to avoid the renal effects of Solutol in studies designed to determine a drugs intrinsic pharmacokinetics, as renal and hepatic clearance could be affected.

Bittner et al. (2003b) reported that Solutol HS15 (5\%, 2ml/kg; $100 \mathrm{mg} / \mathrm{kg}$ ) increased urinary elimination of colchicine in rats, from $1 \%$ of the dose to $39 \%$ over 8 hours. They ascribed this to a toxic effect of Solutol at the kidney. Other effects on colchicine pharmacokinetics were also seen, as described in Chapters 1 and 5 . This increase in renal elimination appears to be in opposition to the effects seen in our study, where elimination of ${ }^{99 \mathrm{~m}}$ Tc-DTPA from the kidneys was delayed by Solutol. However, it is important to note that the effects reported in their study were net effects over 8 hours, whereas our study looked at initial effects on renal elimination, due to our short imaging period. In the work of Azmin et al. (1985) (described below), Tween caused an overall increase in the urinary excretion of methotrexate, but elimination over the first four hours was substantially reduced compared to controls. The overall increase was due to increased urinary elimination compared to the control at later timepoints. It is possible that this is also the case with Solutol; no earlier data from Bittner et al.'s study are reported to enable us to determine this. It is of interest that no such effects were seen when this group investigated the effects of the same dose of Solutol on midazolam pharmacokinetics (Bittner et al., 
2003a). The amount of midazolam eliminated in the urine was $0.003 \%$ in the control group, and was not significantly increased by Solutol treatment, which may have been expected if Solutol had a toxic effect on the kidney. However, midazolam has a high hepatic extraction ratio (Bittner et al., 2003a) and so is efficiently cleared by the liver, which may have prevented any renal effects of Solutol from affecting the pharmacokinetics of this drug.

The increase in $A \cup C_{0-420 s e c}$ seen after dosing with Tween 80 suggests that elimination of ${ }^{99 \mathrm{~m}} \mathrm{Tc}$-DTPA from the kidneys was delayed. The fact that $\mathrm{AUC}_{30-90 \mathrm{sec}}$ was not significantly affected suggests that uptake into the kidneys (i.e. GFR) was not affected by the excipient.

As seen for Solutol, not all of the rats were affected by treatment with Tween. Again, no reason was apparent for this, and it may be that rats differ in their susceptibility to the renal effects of Tween. Our group size is not sufficient to estimate the true incidence of these effects. The fact that renal elimination was not affected in all of the rats could be problematic in pharmacokinetic studies where this dose of Tween is used, as its effects on renal elimination would not be predictable. 
Azmin et al. (1985) reported the effects of $6 \% 5 \mathrm{ml} / \mathrm{kg}$ Tween 80 (equivalent to $319 \mathrm{mg} / \mathrm{kg}$ ) on the renal elimination of methotrexate in mice. The amount of methotrexate excreted unchanged in urine over 24 hours (calculated from their data, assuming mice were maximum weight) was increased from $55 \%$ to $89 \%$ by treatment with the excipient. However, the amount excreted unchanged during the first four hours after methotrexate and Tween dosing was reduced from approximately $31 \%$ to approximately $10 \%$. This initial reduction in the amount of methotrexate appearing in urine (ie. eliminated from the kidneys) fits in with the reduced elimination of ${ }^{99 \mathrm{~m}} \mathrm{Tc}$-DTPA from the kidneys seen in our experiment.

Cummings et al. (1986) reported that Tween increased the urinary excretion of adriamycin in humans. The average amount excreted in urine over 24 hours was increased by 2.2 -fold by Tween treatment. No earlier renal elimination data is reported; it may be that an effect analogous to that described by Azmin et al., with an initial decrease followed by an increase in excretion of unchanged drug into the urine, occurred here. It should be noted that only $1.4 \%$ on average was excreted unchanged into urine in the control group, demonstrating that this is not an important route of elimination for adriamycin. 
Tween 80 is commonly used in pre-clinical studies at doses of $5 \%$ $5 \mathrm{ml} / \mathrm{kg}$, equivalent to $266 \mathrm{mg} / \mathrm{kg}$ (Marcel DeMatas, personal communication). The dose of Tween 80 used in the present studies $(85 \mathrm{mg} / \mathrm{kg})$ is only $32 \%$ of this, and was limited by the volume which could be reliably injected into the penile vein; it was not possible to increase the excipient dose by increasing the concentration of the Tween solution, as the corresponding increase in solution viscosity made injection difficult. However, doses of less than $5 \% 5 \mathrm{ml} / \mathrm{kg}$ are likely to be used in pre-clinical studies if they enable production of a suitable dosing solution. It is possible that the effects seen here would be magnified at higher doses of Tween. The fact that a significant effect of Tween on renal elimination has been demonstrated at a dose which is lower than the maximum used in pre-clinical studies suggests that its potential renal effects should be taken into account when planning pharmacokinetic studies, and interpreting pharmacokinetic data where this excipient has been used.

As discussed for Solutol above, the delayed elimination from the kidney seen with Tween 80 has the potential to affect the pharmacokinetics of drugs which undergo active reabsorption from the kidney, by increasing the amount of time they are available for reabsorption, thus potentially increasing plasma concentrations. This has the potential to affect the renal clearance of drugs, and 
also their hepatic clearance if metabolism or biliary excretion increase to compensate for the reduced renal elimination. It is therefore desirable to avoid the renal effects of this excipient in pharmacokinetic studies.

The reason for the similar effects of Solutol and Tween on renal elimination of ${ }^{99 m}$ Tc-DTPA is unclear. As they are both non-ionic surfactants, and have shown similar effects, they may be acting through a common mechanism. The fact that the effects were seen during our short imaging period, and the excipients were administered only 2-3 minutes before ${ }^{99 \mathrm{~m}}$ TC-DTPA ( 5 minutes for rat A in the Solutol group), demonstrates that this effect occurs quickly. It seems unlikely that the effects seen are due to interaction between the ${ }^{99 \mathrm{~m}} \mathrm{Tc}-\mathrm{DTPA}$ and the excipients (e.g.micellar entrapment), as the excipients were administered separately from the ${ }^{99 m}$ Tc-DTPA, and at different times. After injection, the excipients will have been substantially diluted by the blood. Additionally, Tween 80 is rapidly metabolised by plasma esterases; plasma levels in mice fell to less than $0.05 \% \mathrm{v} / \mathrm{v}, 15$ minutes after administration of a $25 \% 3.3 \mathrm{ml} / \mathrm{kg}$ dose (van Tellingen et al., 1999). Interaction of ${ }^{99 \mathrm{~m}} \mathrm{Tc}$-DTPA with the excipients therefore seems unlikely to be the mechanism for the renal effects seen. It is possible that the excipients somehow increased water reabsorption from the kidneys, thus reducing urinary flow rate and increasing 
${ }^{99 m}$ Tc-DTPA retention in the kidneys. Alternatively, the excipients could be having a toxic effect on the kidneys, causing inflammation of the tubules and thus reducing urinary flow rate. However, these excipients did not increase levels of the acute phase reactant $\alpha_{1}$ AGP in our study at these doses (Chapter 3), demonstrating that they did not bring about sufficient inflammation to induce an acute phase response. Another possible mechanism for these effects is that the excipients, or their breakdown products, could be interacting with cell membrane components to increase the ability of ${ }^{99 \mathrm{~m}}$ Tc-DTPA to enter kidney tubule cells, thus increasing its retention in the kidney.

\subsubsection{Conclusions}

A scintigraphic method has been developed which is suitable for investigating the effects of excipients on the renal elimination of ${ }^{99 m}$ Tc-DTPA, an agent used to determine GFR. Using this method, it has been demonstrated that excipients can affect the renal elimination of ${ }^{99 \mathrm{~m}} \mathrm{Tc}-\mathrm{DTPA}$ at doses likely to be used in pre-clinical studies; significant effects were seen with DMSO (393mg/kg ip), Solutol HS15 (100mg/kg iv), and Tween 80 (85mg/kg iv). Their ability to do this should be taken into consideration when designing formulations for pre-clinical studies, and when interpreting 
pharmacokinetic data from studies where these excipients have been used.

\subsubsection{Further work}

It would be of interest to investigate the effects of excipients on the pharmacokinetics of drugs which have an active transport component to their renal elimination, to complement these studies. It would also be of interest to investigate the effects of the increase in GFR seen with $393 \mathrm{mg} / \mathrm{kg}$ DMSO on total and renal clearance for drugs which undergo both renal and hepatic elimination. Investigation of the impact of the effects seen with Solutol and Tween on the pharmacokinetics of drugs which are reabsorbed from the kidneys would also be of interest, but would be hampered by the fact that these effects did not occur in all rats. It would also be of interest to investigate how long after excipient administration the effects persist. 


\section{Chapter 7:}

Effects of excipients on the

pharmacokinetics of

benzylpenicillin and cimetidine. 


\section{Contents:}

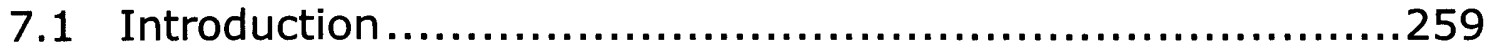

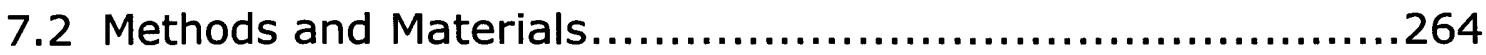

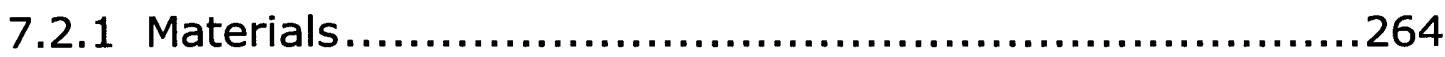

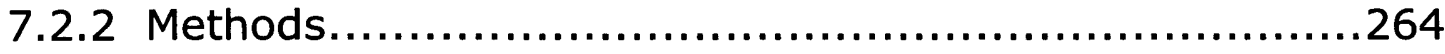

7.3 Results........................................................... 270

7.3.1 Benzylpenicillin ...........................................270

7.3.2 Cimetidine .................................................... 274

7.3.3 Effect of Solutol HS15 on LC/MS/MS analysis ..............281

7.4 Discussion .......................................................... 281

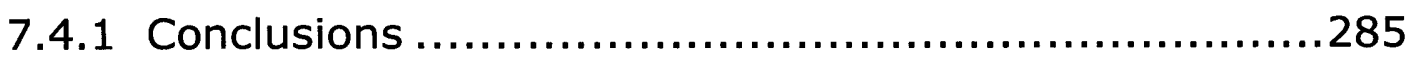

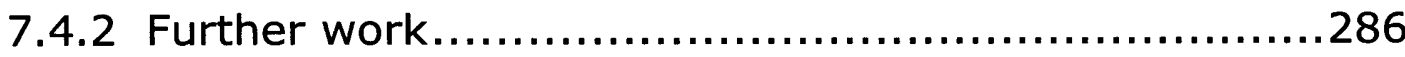




\subsection{Introduction}

As described in the previous chapter, drug elimination in the urine is determined by several processes, predominantly glomerular filtration, active secretion, and reabsorption. In the previous chapter, the potential effects of excipients on GFR were investigated. The experiments described in Chapters 4 and 5 considered the effects of excipients on transport by P-Gp and MRP, both of which are present in renal proximal tubule cells, and are involved in the active renal secretion of their substrates (Inui et al., 2000; Robertson and Rankin, 2006). However, this thesis has yet to consider two of the most important transporters involved in active renal secretion. Organic anion transporters (OATs) and organic cation transporters (OCTs) are located on the basolateral membrane of proximal tubule cells (Inui et al., 2000; Wright, 2005; Robertson and Rankin, 2006). These transporters facilitate the excretion of substrates into the urine, by transporting them from the blood into the proximal tubule cells.

OATs transport a variety of drug substrates, including $\beta$-lactam antibiotics, antivirals, NSAIDs, and methotrexate (Jariyawat et al., 1999; Inui et al., 2000; Robertson and Rankin, 2006). The importance of their role in renal elimination is demonstrated by the 
well-known interaction between penicillins and probenecid. Both drugs are OAT substrates, and compete for tubular secretion by OATs in the kidney (Tsuji et al., 1990). This reduces the renal clearance of penicillins, giving rise to increased plasma levels (Overbosch et al., 1988). This demonstrates that inhibition of active renal secretion can have a significant impact on drug pharmacokinetics.

No investigations into the effects of excipients on OATs are reported in the literature. This is therefore an important area to investigate. Determining the effects of excipients on the plasma pharmacokinetics of drugs which are substrates for OATs appears to be a useful method for such investigations, as demonstrated by the interaction described above. Significant inhibition of the transporters would be expected to reduce the active renal secretion of their substrates, and hence reduce their renal clearance and potentially total clearance.

Benzylpenicillin is transported by OATs, and has been shown to be a substrate for rOAT3 and rOAT1 (Jariyawat et al., 1999; Hasegawa et al., 2003), although rOAT3 is thought to be more important in its renal elimination (Hasegawa et al., 2003). The interaction with probenecid described above demonstrates that inhibition of OATmediated renal secretion significantly affects the pharmacokinetics 
of this compound. This suggests that benzylpenicillin is a useful substrate to investigate the effects of excipients on OATs in vivo, as substantial inhibition of the transporter by excipients could be expected to have a significant and detectable effect on the plasma concentration profile. The volume of blood that can be sampled in pharmacokinetic experiments is limited due to license restrictions and the need to maintain physiological normality. This effectively limits the number of plasma samples which can be taken, in order to ensure that each sample is of sufficient volume for analysis. An additional advantage of using benzylpenicillin is its simple pharmacokinetic profile, which should be possible to adequately characterise within our sampling limitations.

Substrates for OCTs include $\mathrm{H}_{2}$ receptor antagonists, procainamide, quinidine and tetraethylammonium (Inui et al., 2000; Tahara et al., 2005; Wright, 2005). As described above for OATS, it should be possible to investigate the effects of excipients on OCTs by determining their effects on plasma pharmacokinetics of an OCT substrate. Cimetidine is transported by both OATs and OCTs, having been shown to be a substrate for hOATs1-3, rOAT3, hOCT2 and rOCT1\&2 (Kusuhara et al., 1999; Cha et al., 2001; Motohashi et al., 2004; Tahara et al.,2005; Ueo et al.,2005). 
The importance of OCT2 in the renal clearance of cimetidine was demonstrated by Ji et al. (2002). They demonstrated that rOCT2 was downregulated in 5/6 nephrectomised rats (per mass of renal tissue), with no change in the expression of rOAT1, rOAT3 and rOCT1, and that cimetidine total and renal clearance was also reduced in these rats. Some of the reduction in cimetidine clearance seen in their study is likely to be due to the loss of renal tissue by nephrectomy rather than reduced rOCT2 expression. However, cimetidine renal secretory clearance per mass of tissue was also reduced in these rats, and increasing rOCT2 expression by testosterone treatment partially restored cimetidine clearance. This demonstrates that rOCT2 is important in the renal clearance of cimetidine, and suggests that the reduction in clearance seen in the nephrectomised rats was partially due to the downregulation of rOCT2, not just loss of renal tissue.

The fact that reduced active secretion of cimetidine, due to reduced rOCT2 expression, had a significant effect on its plasma pharmacokinetics in the study described above (Ji et al., 2002) suggests that significant inhibition of OCT2 could also have a discernible effect on the pharmacokinetic profile of cimetidine. Cimetidine therefore appears to be a useful substrate to investigate the effects of excipients on OCT2 in vivo. Additionally, the plasma 
concentration-time profile of cimetidine should be possible to accurately characterise within our sampling limitations.

Time and resource limitations mean that only one excipient can be investigated in this set of experiments. As Solutol HS15 showed significant effects on transport by MRP and possibly P-Gp in vivo. (as demonstrated in Chapter 5), it was decided that it would be of interest to investigate its in vivo effects on OATs and OCTs. This series of experiments therefore aims to investigate the effects of Solutol HS15 on OATs and OCTs in vivo, by determining its effects on the pharmacokinetics of benzylpenicillin and cimetidine. 


\subsection{Methods and Materials}

\subsubsection{Materials}

Cimetidine, benzylpenicillin sodium salt, ranitidine hydrochloride, phenoxymethylpenicillinic acid potassium salt and EDTA were obtained from Sigma, UK. $\mathrm{HCl}$ was obtained from Fisher, UK. Solutol HS15 was a kind gift from AstraZeneca, Alderley Park, UK. Acetonitrile, formic acid and blank rat plasma were obtained from AstraZeneca, Alderley Park, UK.

\section{Animals}

Male Sprague-Dawley rats (240-310g) (Harlan) were group housed on a 12 hour light/dark cycle, with access to water and standard laboratory rodent diet ad libitum.

\subsubsection{Methods}

Rats were initially sedated with $0.3 \mathrm{ml}$ Hypnorm (fentanyl citrate $0.315 \mathrm{mg} / \mathrm{ml}$ and fluanisone $10 \mathrm{mg} / \mathrm{mg}$, VetaPharma) i.p. Further doses of Hypnorm were administered as necessary. Intravenous 
injections were carried out under isoflurane. Isoflurane was also used to assist in obtaining venous samples where necessary.

Each control or treatment group consisted of four rats. A pre-dose venous sample (approx. $0.18 \mathrm{ml}$ ) was obtained after initiation of Hypnorm anaesthesia, but before administration of the study drugs or excipients. Benzylpenicillin $(20 \mathrm{mg} / \mathrm{kg}$ ) or cimetidine $(10 \mathrm{mg} / \mathrm{kg})$ were administered by i.v. injection $(0.8 \mathrm{ml} / \mathrm{kg}$ dose volume). Benzylpenicillin was dissolved in $0.9 \% \mathrm{NaCl}$. Cimetidine dosing solution was prepared by dissolving the drug in $0.1 \mathrm{M} \mathrm{HCl}(3 \mathrm{ml}$ per $62.5 \mathrm{mg}$ of drug), then diluting with $0.9 \% \mathrm{NaCl}$ to give an injection volume of $0.8 \mathrm{ml} / \mathrm{kg}$. Rats in the Solutol treatment groups also received $100 \mathrm{mg} / \mathrm{kg}$ Solutol HS15 i.v. (administered as a $12.5 \%$ solution, $0.8 \mathrm{ml} / \mathrm{kg}), 1.5-2$ minutes prior to injection of the dose. Venous samples (approx. $0.18 \mathrm{ml}$ ) were obtained and centrifuged (12000 rcf, 5 minutes) to separate plasma. A 5\% solution of EDTA (33-36 $\mu \mathrm{l}$ per $\mathrm{ml}$ ) was used as anticoagulant. Plasma samples and aliquots of dosing solution were frozen until analysis. The penile vein was used for dosing; blood was sampled from the tail vein.

\section{Sample analysis - LC/MS/MS}

\section{Sample preparation}

$50 \mu l$ of each sample (or $25 \mu l+25 \mu$ l blank plasma, for those expected to be of high concentration) were transferred into glass 
tubes. Internal standard was added (phenoxymethylpenicillinic acid potassium salt for benzylpenicillin $(50 \mu \mathrm{l}$ of a $0.5 \mu \mathrm{g} / \mathrm{ml}$ solution in of 50:50 ACN: $\left.\mathrm{H}_{2} \mathrm{O}\right)$, ranitidine $\mathrm{HCl}$ for cimetidine $(25 \mu \mathrm{l}$ of a $0.5 \mu \mathrm{g} / \mathrm{ml}$ solution in of 50:50 ACN: $\left.\mathrm{H}_{2} \mathrm{O}\right)$ ), and $50 \mu$ l of 50:50 ACN: $\mathrm{H}_{2} \mathrm{O}$ was added to each tube. $300 \mu$ l of ACN was added to precipitate proteins, and tubes were thoroughly vortex mixed. Tubes were centrifuged (MSE Falcon $6 / 300$ centrifuge, $4500 \mathrm{rpm}, 4^{\circ} \mathrm{C}$ for 10 mins; or Sanyo Mistral $3000 \mathrm{i}$ centrifuge, $3600 \mathrm{rcf}, 18^{\circ} \mathrm{c}$ for $10 \mathrm{mins}$ ) and an aliquot of the supernatant transferred to HPLC vials. Calibration standards were prepared using blank plasma in the range $0.1-10 \mu \mathrm{g} / \mathrm{ml}$ for benzylpenicillin and $0.05-10 \mu \mathrm{g} / \mathrm{ml}$ for cimetidine, and are shown in Figures 7.1 and 7.2. Some of the benzylpenicillin samples had concentrations outside the calibration range, and so were diluted with 50:50 ACN: $\mathrm{H}_{2} \mathrm{O}$ and re-analysed.

\section{Analysis}

The LC/MS/MS system consisted of a HP1100 Series HPLC and Applied Biosystems MDS SCIEX API4000 mass spectrometer, with CTC analytics HTC PAL autosampler and Analyst software (Applied Biosystems MDS SCIEX). An Atlantis $\mathrm{dC}_{18} 3 \mu \mathrm{m}, 4.5 \times 50 \mathrm{~mm}$ column was used, with $40 \% \mathrm{ACN} / 54 \% \mathrm{H}_{2} \mathrm{O} / 6 \%$ formic acid solution (2.5\%) mobile phase and $1.2 \mathrm{ml} / \mathrm{min}$ flow rate. The mass spectrometer was used in turbo ion spray mode; optimised working parameters for detection of benzylpenicillin and cimetidine are shown in Tables 7.1 
and 7.2. Ionisation modes and mass transitions for drugs and internal standards are shown in Table 7.3.

Table 7.1: Optimised working parameters for benzylpenicillin.

\begin{tabular}{|l|c|}
\hline \multicolumn{1}{|c|}{ Parameter } & Value \\
\hline Curtain gas (psi) & 12 \\
\hline Nebuliser gas (psi) & 55 \\
\hline Auxiliary gas (psi) & 50 \\
\hline lonisation voltage & -2500 \\
\hline Evaporation temperature $\left({ }^{\circ} \mathrm{c}\right)$ & 700 \\
\hline $\begin{array}{c}\text { Collision assisted dissociation } \\
\text { (psi) }\end{array}$ & 4 \\
\hline
\end{tabular}

Table 7.2: Optimised working parameters for cimetidine.

\begin{tabular}{|l|c|}
\hline \multicolumn{1}{|c|}{ Parameter } & Value \\
\hline Curtain gas (psi) & 14 \\
\hline Nebuliser gas (psi) & 60 \\
\hline Auxiliary gas (psi) & 60 \\
\hline lonisation voltage & 3500 \\
\hline Evaporation temperature $\left({ }^{\circ} \mathrm{C}\right)$ & 750 \\
\hline Collision assisted dissociation $(\mathrm{psi})$ & 6 \\
\hline
\end{tabular}

Table 7.3: Ionisation modes and mass transitions for drugs and internal standards (IS).

\begin{tabular}{|l|c|c|}
\cline { 2 - 3 } \multicolumn{1}{c|}{} & $\begin{array}{c}\text { Ionisation } \\
\text { mode }\end{array}$ & Mass transition \\
\hline Benzylpenicilin & Negative & $333.2 / 192.2$ \\
\hline Phenoxymethylpen. (IS) & Negative & $349.0 / 207.8$ \\
\hline \hline Cimetidine & Positive & $253.1 / 159.3$ \\
\hline Ranitidine (IS) & Positive & $315.0 / 175.9$ \\
\hline
\end{tabular}




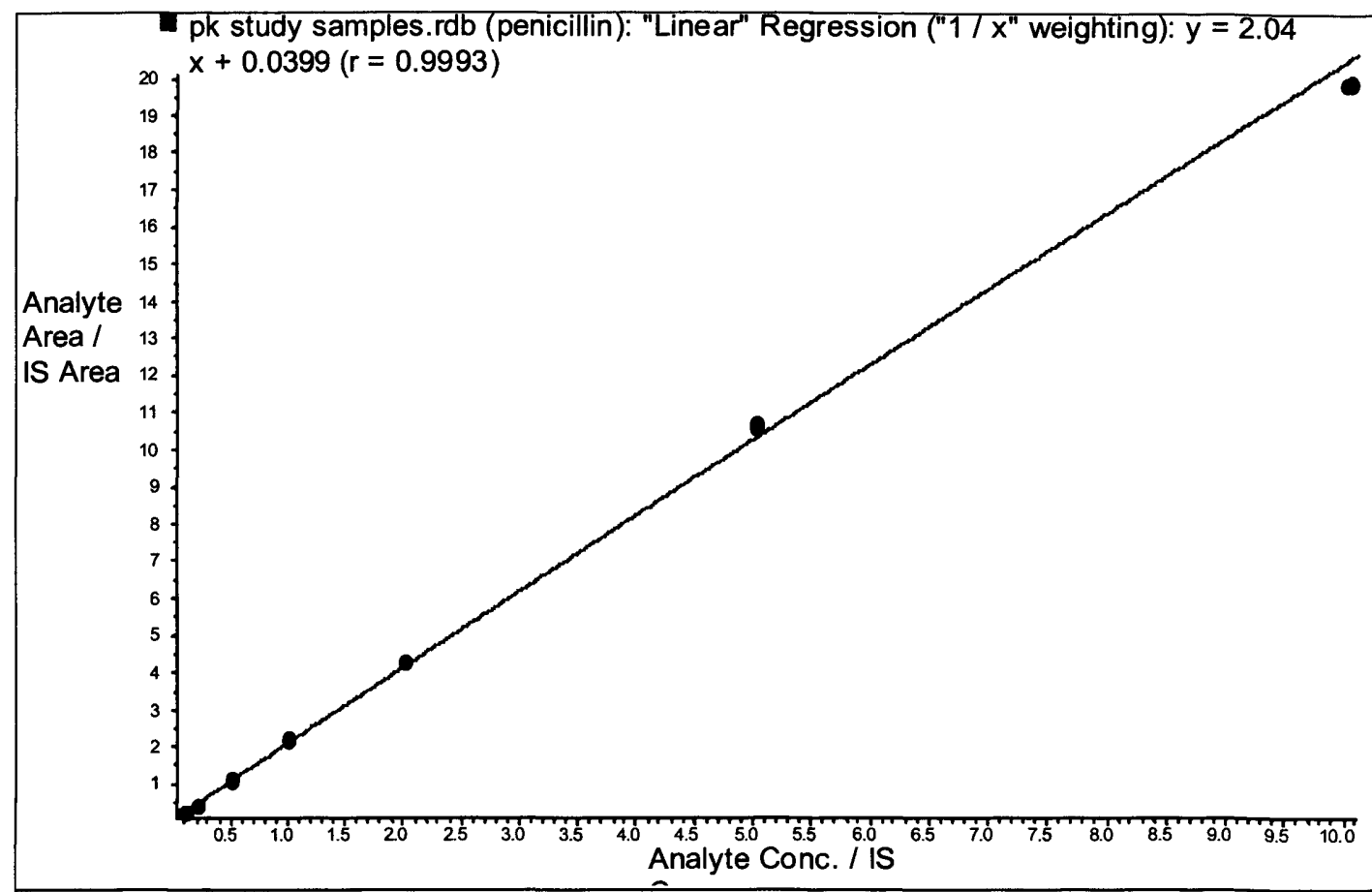

Figure 7.1: LC/MS/MS calibration line obtained for benzylpenicillin.

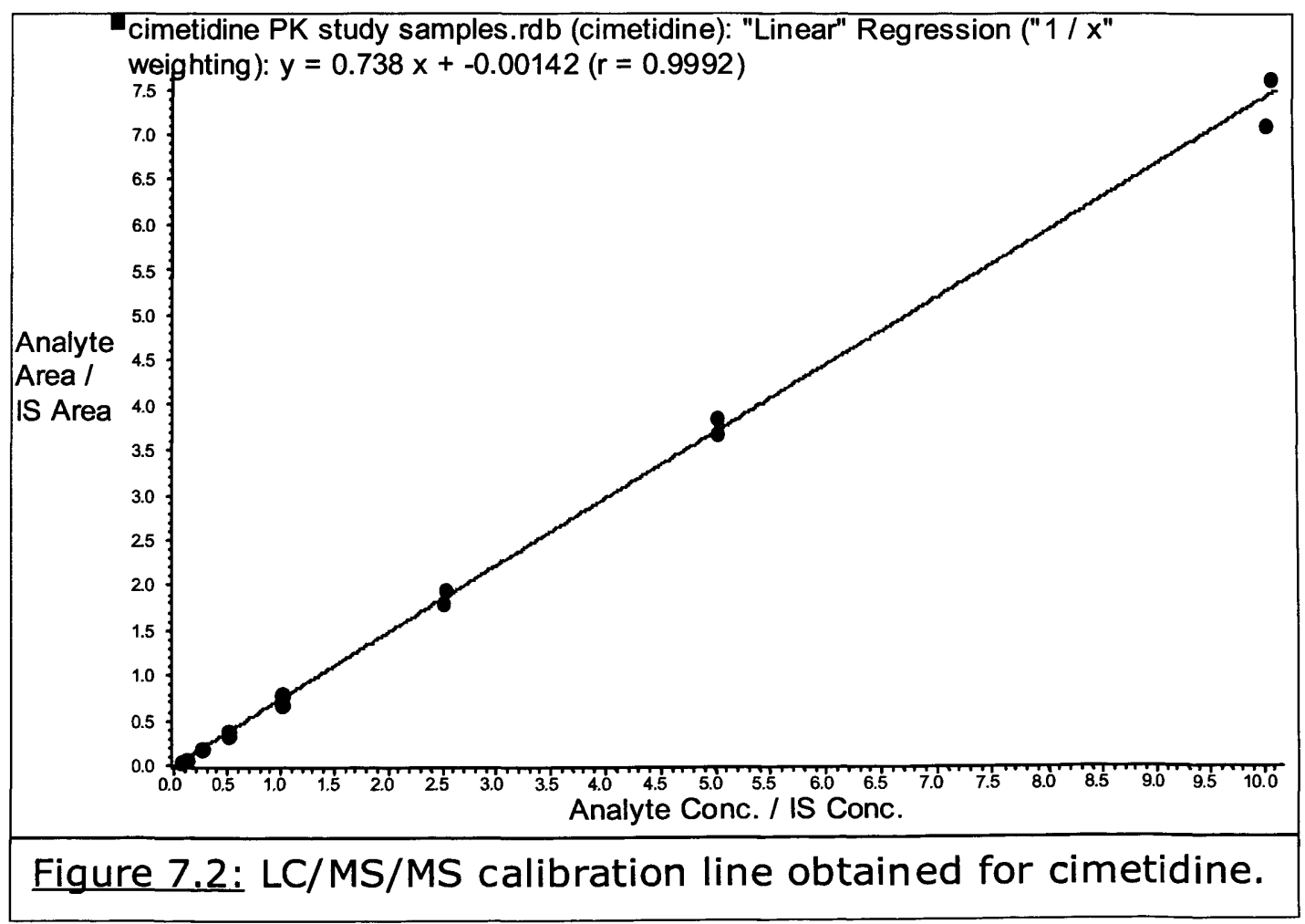


Sample chromatographs obtained from extracted plasma samples are shown in Figures 7.3 and 7.4.

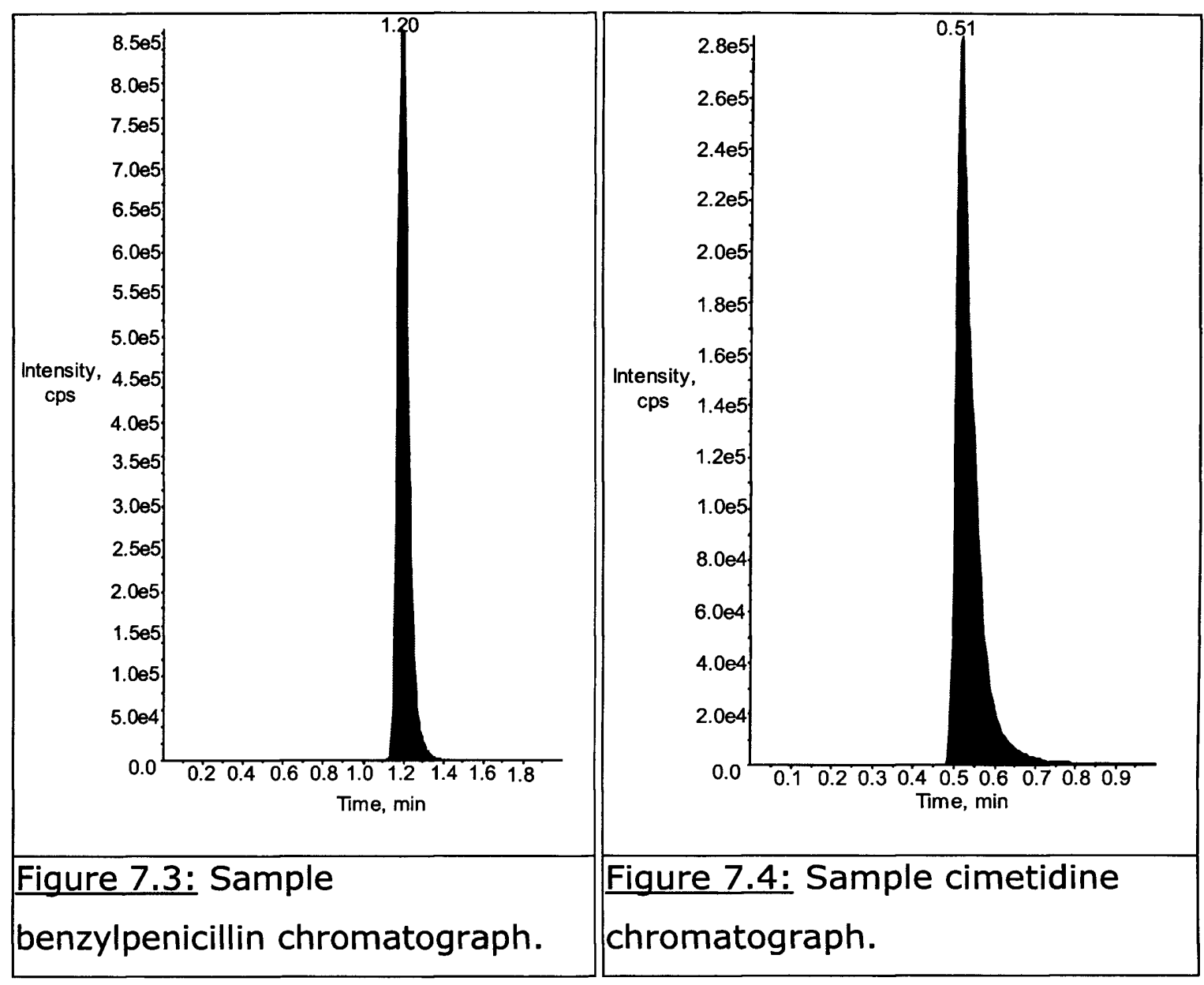

\section{Data analysis}

Plasma concentration data were modelled using WinNonLin (Pharsight). Data for each rat were fitted individually, using a 1compartment open model for benzylpenicillin $\left(C=C_{0} \cdot e^{-k t}\right)$, and a 2compartment open model for cimetidine $\left(C=A e^{-\alpha t}+B e^{-B t}\right)$, with $1 /$ predicted y weighting. Statistical significance was assessed using a one-way ANOVA, performed using SPSS (SPSS Inc.). 


\subsection{Results}

\subsubsection{Benzylpenicillin}

Figures 7.5 and 7.6 show the benzylpenicillin plasma concentration vs. time curves for control and Solutol treated rats. Pharmacokinetic parameters calculated for individual rats are shown in Table 7.4. There were no statistically significant differences between the control and Solutol treatment groups for any of the parameters.

A small amount of the benzylpenicillin dose was lost during injection of Rat $\mathrm{D}$ in the control group, and a small amount was retained at the injection site for Rat E in the Solutol group. As it was not possible to accurately quantify these amounts, these factors were not taken into account in calculation of the pharmacokinetic parameters. Although Rat D does not appear to be an outlier, the clearance obtained in Rat $\mathrm{E}$ is the highest in the treatment group. However, even if data from this rat are excluded from the statistical analysis, there are still no statistically significant differences between the control and Solutol treatment groups. Surprisingly, no clearance values for benzylpenicillin in the rat appear to be reported 
in the literature for comparison with the data obtained in the present study. 

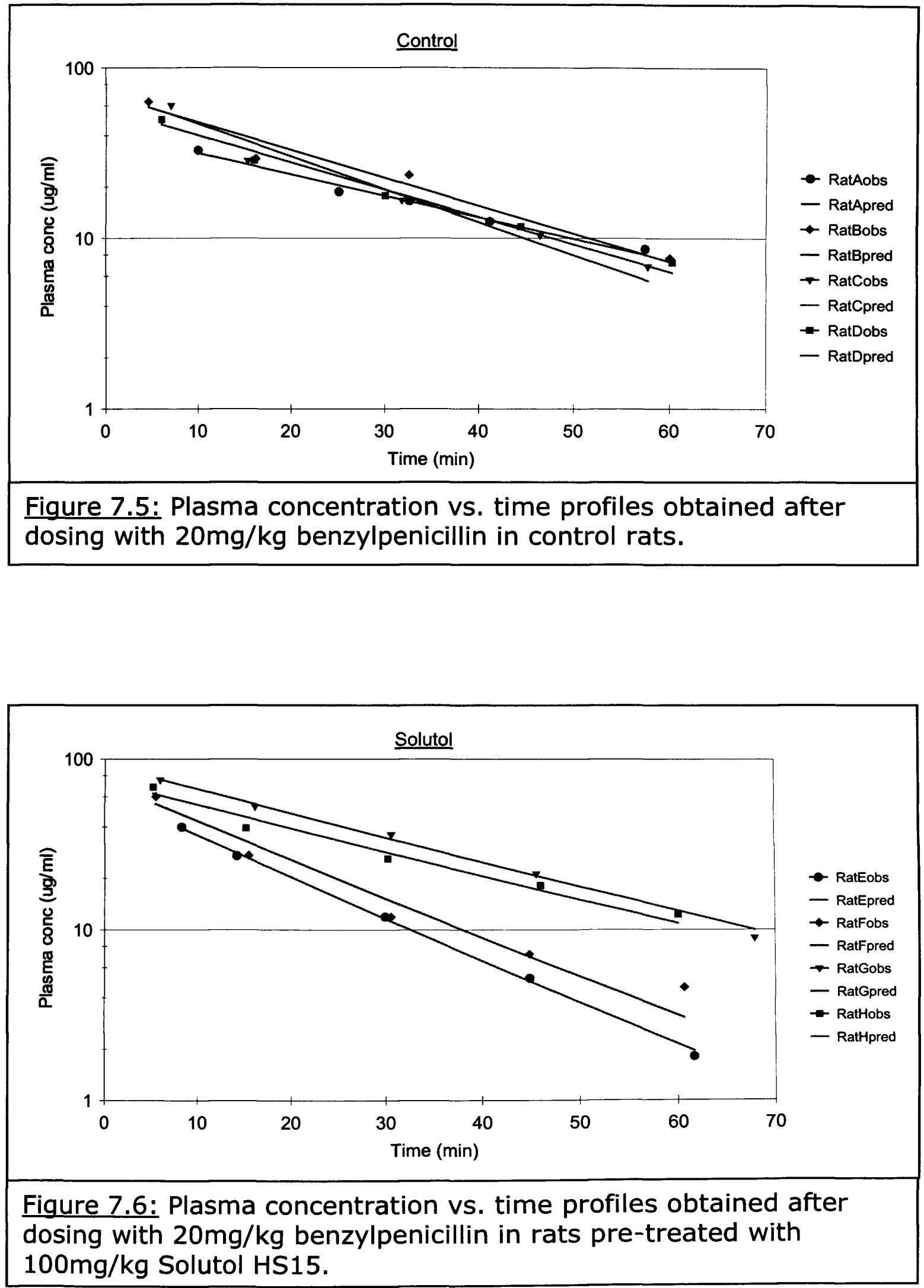


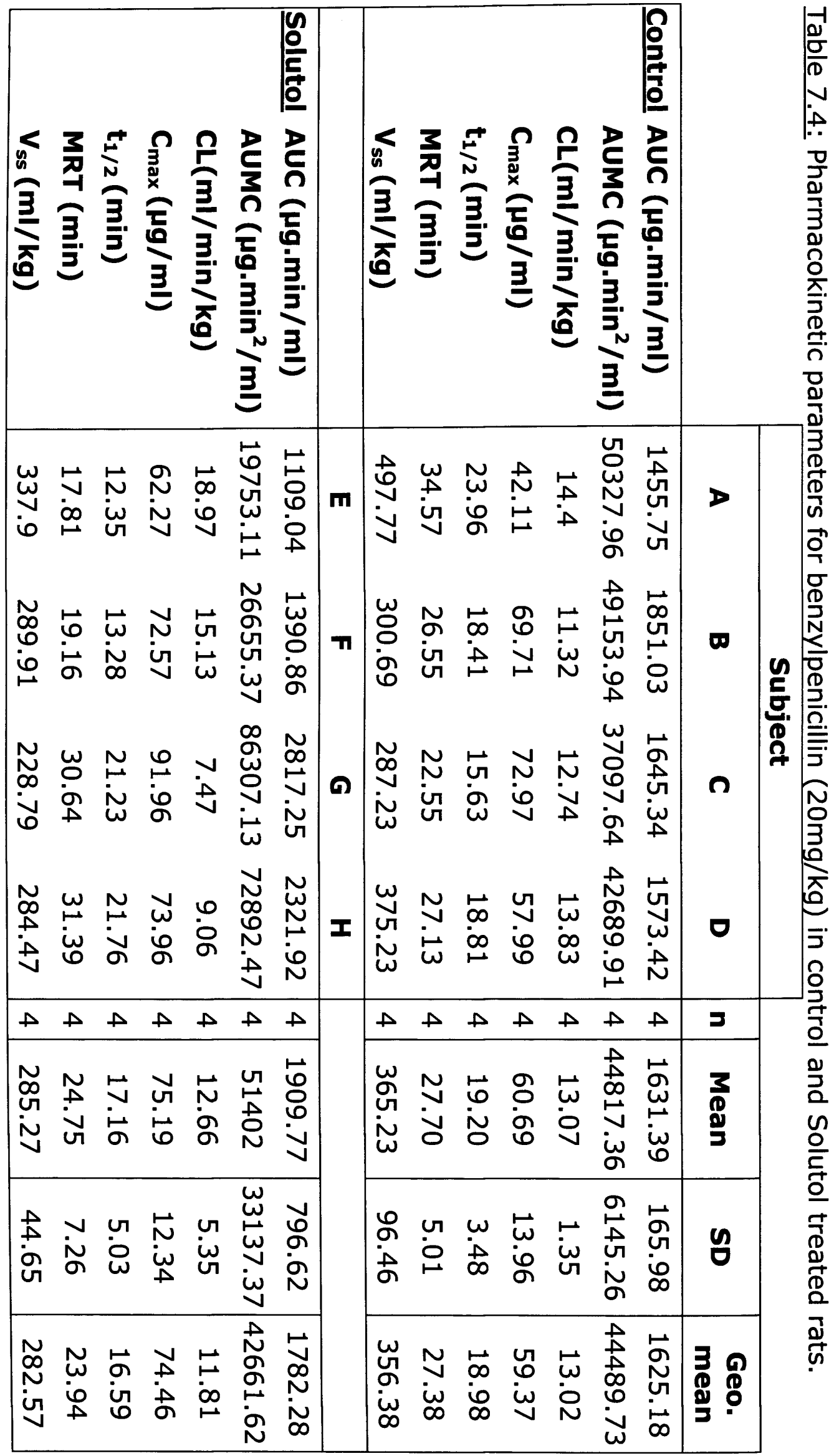




\subsubsection{Cimetidine}

Figures 7.7 and 7.8 show the cimetidine plasma concentration vs. time curves for control and Solutol treated rats. Pharmacokinetic parameters calculated for individual rats are shown in Table 7.5. There were no statistically significant differences between the control and Solutol treatment groups for any of the parameters. A small amount of the cimetidine dose was lost during injection of Rat $\mathrm{K}$ in the control group; as this could not be accurately quantified, it was not taken into account when calculating the pharmacokinetic parameters

The concentration of the cimetidine dosing solutions measured by LC/MS/MS was 5.4-5.9 times higher than expected - concentrations of 6.8 and $7.4 \mu \mathrm{g} / \mathrm{ml}$ were measured, instead of the $1.25 \mu \mathrm{g} / \mathrm{ml}$ which would be expected from a $1 / 10000$ dilution of the $12.5 \mathrm{mg} / \mathrm{ml}$ dosing solution. No reason could be discerned for this. It did not appear to be due to a problem with the detection method or equipment, as the concentrations of standard solutions were measured as expected. New dilutions of the dosing solutions were prepared and analysed, but gave essentially the same result. The pipettes and volumetric flasks used to perform the dilutions are unlikely to be the cause, as they were also used in the dilution of 
the benzylpenicillin dosing solutions, where no such problem occurred, and the pipettes had been recently calibrated. It is also unlikely to be due to an error in performing the dilutions, as the repeat dilutions were observed by another worker, and the calculations for this dilution were independently checked.

Nor does there appear to have been an error in the preparation of the dosing solutions. Balance readings were recorded during preparation of each solution, so the correct amount appears to have been weighed. Also, dosing solutions were freshly prepared each day, and it would be extremely unlikely to misread the balance in the same way on two separate occasions. The balance itself does not appear to have been in error, as it was also used in preparation of the benzylpenicillin dosing solutions, and was also in use by other workers who did not experience any problems. Nor does it appear possible that the wrong size volumetric flask was used to dissolve the cimetidine; use of a $2 \mathrm{ml}$ instead of a $5 \mathrm{ml}$ flask would not have provided sufficient volume for dosing and LCMSMS analysis. It also appears unlikely that the $\mathrm{HCl}$ used in preparation of the cimetidine dosing solution interfered with the analysis, due to the large dilutions used. It may be that the difference between calculated and measured dosing solution concentrations is due to a dilution effect. However this would not be expected, as two separate $1 / 100$ 
dilutions were performed. There is therefore no apparent explanation for this discrepancy. However, as it occurred in both the control and Solutol dosing experiments, it is still possible to compare results from the two groups to determine any effect of excipient treatment.

Comparison of the values for clearance obtained in the present study, using either the calculated or measured dosing solution concentration, with values from the literature may give an insight into which concentration is correct. Cimetidine has dose-dependent pharmacokinetics, and its clearance decreases with increasing dose (Adedoyin et al., 1987; McNamara et al., 1992). Adedoyin et al. (1987) reported clearances of $68.5,57.3$ and $36.8 \mathrm{ml} / \mathrm{min} / \mathrm{kg}$ at doses of 10,40 and $100 \mathrm{mg} / \mathrm{kg}$ in the rat. McNamara et al. (1992) reported clearances of $40.7,39.3$ and $26.6 \mathrm{ml} / \mathrm{min} / \mathrm{kg}$, during infusions of $0.4,2$ and $10 \mathrm{mg} / \mathrm{hr}$ in lactating rats (converted from $\mathrm{ml} / \mathrm{min}$ value using mid-point of weight range). Hendersen et al. (1988) reported a cimetidine clearance of $35.6 \mathrm{ml} / \mathrm{min} / \mathrm{kg}$, after a $10 \mathrm{mg} / \mathrm{kg}$ loading dose plus $40 \mu \mathrm{g} / \mathrm{min}$ infusion. Ji et al. (2002) reported a total clearance of $17.2 \mathrm{ml} / \mathrm{min} / \mathrm{kg}$ in sham-operated rats after a loading dose of $80 \mathrm{mg} / \mathrm{kg}$, plus $12 \mathrm{mg} / \mathrm{hr}$ infusion. Yeganeh and Ramzan (2002) reported a clearance of $26 \mathrm{ml} / \mathrm{min} / \mathrm{kg}$ in rats after a dose of $15 \mathrm{mg} / \mathrm{kg}$ cimetidine. All of the above clearance 
values were obtained in Sprague-Dawley rats, the same strain as used in the present study, with the exception of Ji et al. (2002), where Wistar rats were used. Use of the LC/MS/MS measured dosing solution concentrations (corresponding to a dose of $60 \mathrm{mg} / \mathrm{kg}$ ) gives clearances of 92.40 and $109.11 \mathrm{ml} / \mathrm{min} / \mathrm{kg}$ for the control and Solutol treatment groups in the present study, compared to clearances of 15.61 and $18.43 \mathrm{ml} / \mathrm{min} / \mathrm{kg}$ when the expected dose $(10 \mathrm{mg} / \mathrm{kg})$ is used. While the clearances obtained using the LC/MS/MS measured dosing solution concentration appear to be too high, the clearances obtained using the expected doses are lower than would be expected.

The volume of distribution is another useful a parameter to compare in order to determine whether the measured dosing solution concentration is correct, as the calculated volume will be affected by the dose value used. Adedoyin et al. (1987) reported volumes of distribution (V) ranging from $2.0-2.61 / \mathrm{kg}$, which did not differ significantly with dose, while Yeganeh and Ramzan (2002) reported a value of $2.61 / \mathrm{kg}$ for $\mathrm{V}_{\mathrm{ss}}$. Use of the LC/MS/MS measured dosing solution concentrations gives values of $7.8-8.21 / \mathrm{kg}$ for $V_{s s}$ for the control and Solutol treatment groups respectively, compared to 1.3$1.41 / \mathrm{kg}$ when the expected dose is used. For this parameter, the values obtained using the expected dose are clearly closer to the 
published values than those obtained when the dose measured by LC/MS/MS is used. It therefore appears that the expected dose is correct, and the unexpectedly high dosing solution concentrations measured by LC/MS/MS are an artefact, due to unidentified experimental or measurement error. The values obtained using the expected doses were therefore chosen for inclusion in Table 7.5. 

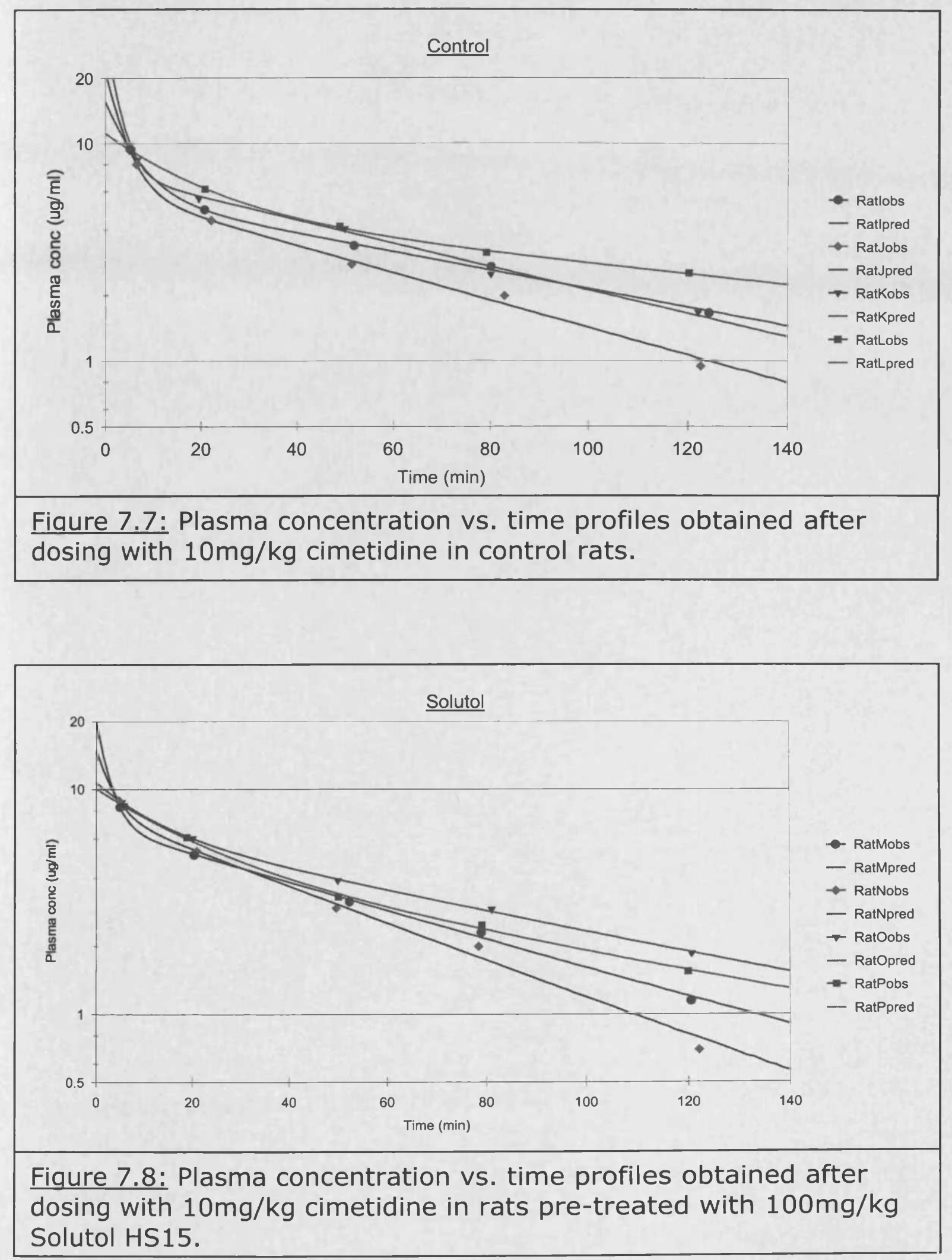


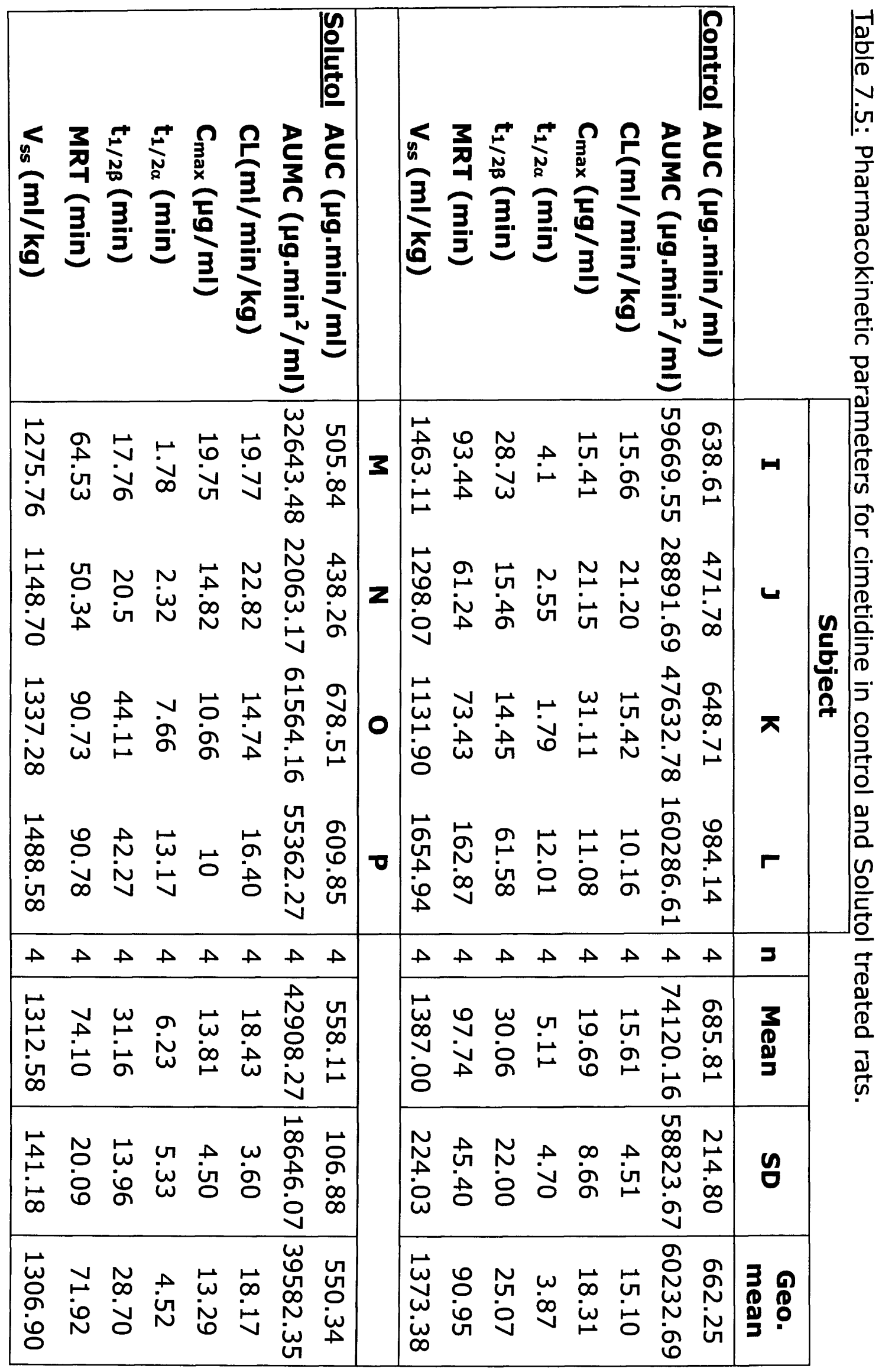




\subsubsection{Effect of Solutol HS15 on LC/MS/MS analysis}

A sample of plasma from a rat dosed with Solutol HS15 and Hypnorm but no drug was run on both LC/MS/MS methods. No peaks which would interfere with the detection of either of the compounds under investigation or the internal standards were detected.

\subsection{Discussion}

Treatment with $100 \mathrm{mg} / \mathrm{kg}$ Solutol HS15 did not significantly affect the plasma pharmacokinetics of either cimetidine or benzylpenicillin. This suggests that the excipient did not substantially affect their active renal secretion by either OATs or OCTs.

It is also possible, however, that an effect did occur, but was not detectable by the methods used in the present study. It is possible that the compounds chosen for the pharmacokinetic studies are unsuitable for investigating inhibition of OATs and OCTs in vivo. Neither of the compounds used in the present study is exclusively eliminated by the kidney. Benzylpenicillin has been reported to 
undergo $79 \%$ renal clearance in rats (Kortopolous et al., 1983). Adedoyin et al. (1987) reported that cimetidine underwent $37 \%$ renal clearance in rats at a dose of $10 \mathrm{mg} / \mathrm{kg}$, and $45 \%$ at 40 and $100 \mathrm{mg} / \mathrm{kg}$. Henderson et al. (1988) reported a higher value of $68 \%$ renal clearance for cimetidine (loading dose of $10 \mathrm{mg} / \mathrm{kg}$ plus $40 \mu \mathrm{g} / \mathrm{min}$ infusion), while Ji et al. (2002) reported a value of $81 \%$ (loading dose $80 \mathrm{mg} / \mathrm{kg}$ plus $12 \mathrm{mg} / \mathrm{hr}$ infusion) in sham-operated rats. The latter two values were obtained in rats under surgical anaesthesia, which may be the reason they are so much higher than those reported by Adedoyin et al. (1987). It is possible that any reduction in renal clearance in the present studies, caused by inhibition of active renal secretion, could be masked by a compensatory increase in hepatic clearance, thus preventing an effect from being detected when only plasma clearance is determined.

The compounds used in the present study, however, were chosen because reductions in active renal secretion have been shown to substantially affect their plasma pharmacokinetics. The well-known clinical interaction between penicillins and probenecid, mediated by competitive inhibition of OATs, demonstrates that inhibition of active renal secretion can significantly affect benzylpenicillin pharmacokinetics (Overbosch et al., 1988). This interaction appears to occur in rats as well as humans; increased plasma concentrations 
of ${ }^{14} \mathrm{C}$-benzylpenicilin after probenecid administration in rats were reported by Bergholz et al. (1980), although this was attributed to reduced liver uptake as well as kidney uptake. It would therefore be expected that significant inhibition of OATs by Solutol HS15 would have had a detectable effect on benzylpenicillin pharmacokinetics in the present study.

The work of Ji et al. (2002) demonstrates the importance of rOCT2 in the renal elimination of cimetidine, and suggests that inhibition of rOCT2 could be expected to affect the plasma pharmacokinetics of cimetidine. They demonstrated that rOCT2 was downregulated in 5/6 nephrectomised rats (per mass of renal tissue), whereas expression of rOAT1, rOAT3 and rOCT1 was unchanged. Total and renal clearance of cimetidine were also reduced in the nephrectomised rats, to $63 \%$ and $16 \%$ of the values in shamoperated controls. Some of this reduction in cimetidine clearance is likely to be due to loss of renal mass by $5 / 6$ nephrectomy, rather than just downregulation of rOCT2. However renal secretory clearance per mass of tissue was also reduced, to $14 \%$ of the control value. The authors hypothesised that the selective downregulation of rOCT2 was due to lower levels of testosterone in the nephrectomised rats, and proceeded to determine the effects of exogenous testosterone on rOCT2 expression and cimetidine clearance. Nephrectomised rats dosed with exogenous testosterone 
control value, while total clearance increased from $48 \%$ to $60 \%$.

Renal secretory clearance (per mass of tissue) increased from $5 \%$ to $62 \%$ of the control value. However, testosterone treatment had no effect on cimetidine pharmacokinetics in sham operated rats. Thus, increasing rOCT2 expression in the nephrectomised rats partially reinstated cimetidine clearance compared to non-treated nephrectomised rats. This demonstrates that rOCT2 is important in the renal clearance of cimetidine, and suggests that the reduction in clearance seen in the nephrectomised rats compared to controls was partially due to the downregulation of rOCT2, not just loss of renal tissue. Although the 3 -fold increase in renal clearance after testosterone treatment only translated to a $25 \%$ increase in total clearance, this was still significant, and does not necessarily represent the maximum possible change in clearance caused by rOCT2 inhibition in intact rats. The results of the work of Ji et al. (2002) therefore suggest that substantial inhibition of rOCT2 by Solutol should have had a detectable effect on cimetidine plasma pharmacokinetics in the present study. 
Collection of urine would have enabled the renal clearance of the compounds to be calculated, which may have enabled any effects on renal clearance which were not substantial enough to affect overall plasma clearance to be detected. The small volumes of urine produced by the rat mean that only one sample of sufficient volume is likely to be obtained during the 1-2 hour experimental period. This would only enable overall changes in urinary elimination to be determined, rather than changes in the time course of urinary elimination. Collection of urine in conscious rats requires use of a metabolism cage-type apparatus; however, these are associated with large losses of compound. Bladder catheterisation enables complete collection of urine, but requires surgical anaesthesia, which may affect drug pharmacokinetics. For these reasons, urine collection was not attempted in the present experiments. However, it may be worth considering adding this to the design of future studies in this area.

\subsubsection{Conclusions}

Solutol HS15, at a dose of $100 \mathrm{mg} / \mathrm{kg}$, did not significantly affect the pharmacokinetics of benzylpenicillin or cimetidine. This suggests that it does not inhibit OATs or OCTs in vivo, to an extent which can significantly affect the disposition of these drugs. 


\subsubsection{Further work}

The effects of other excipients on OATs and OCTs could be investigated in cell lines over-expressing these transporters. This would enable the identification of excipients which had significant effects in vitro, and would be suitable for further investigation in in vivo pharmacokinetic studies. Inclusion of Solutol HS15 in such experiments would confirm whether this excipient does not affect these transporters, or whether a small effect may be present which the methods used in the present study did not detect. Design of the in vivo experiments could then be modified if this were the case, for example by the addition of urine collection. 
Chapter 8:

Discussion 
There is a lack of in vivo data in the literature on the effects of excipients on drug disposition, using doses commonly used in early pharmacokinetic studies. The present studies were therefore undertaken to further investigate this area. The data obtained in this thesis demonstrate that excipients can significantly affect drug disposition, at doses commonly used in early pharmacokinetic studies. This highlights the importance of considering potential effects of excipients on pharmacokinetics when designing formulations for use in pre-clinical studies, and when interpreting results from studies where these excipients have been used. The information obtained on which excipients affected specific aspects of drug disposition will be useful in the rational design of formulations, enabling the excipients least likely to affect the pharmacokinetics of the drug under investigation to be selected for use. Table 8.1 summarises which excipients affected the various aspects of drug disposition investigated in each of the chapters. 
Table 8.1: Effects of excipients on drug disposition in each chapter.

\begin{tabular}{|l|c|c|c|c|c|c|}
\cline { 2 - 7 } \multicolumn{1}{l|}{} & DMSO & PG & PEG & $\begin{array}{c}\text { HP- } \beta- \\
\text { CD }\end{array}$ & $\begin{array}{c}\text { Solutol } \\
\text { Breath tests }\end{array}$ & $\begin{array}{c}\text { Tween } \\
80\end{array}$ \\
\hline $\begin{array}{l}\text { Chapter 3: } \alpha_{1}{ }^{-} \\
\text {AGP levels }\end{array}$ & $\mathrm{Y}$ & $\mathrm{Y}$ & - & - & $\mathrm{N}$ & - \\
\hline $\begin{array}{l}\text { Chapter 4: In } \\
\text { vitro P-Gp }\end{array}$ & $\mathrm{N}$ & $\mathrm{Y}$ & $\mathrm{Y}$ & - & $\mathrm{Y}$ & $\mathrm{Y}$ \\
\hline $\begin{array}{l}\text { Chapter 5: In } \\
\text { vivo MIBI/HIDA }\end{array}$ & - & - & - & - & $\mathrm{Y}$ & $\mathrm{Y}$ \\
\hline $\begin{array}{l}\text { Chapter 6: Renal } \\
\text { elimination }\end{array}$ & $\mathrm{Y}$ & $\mathrm{N}$ & - & - & $\mathrm{Y}$ & $\mathrm{Y}$ \\
\hline $\begin{array}{l}\text { Chapter 7: PK of } \\
\text { benzylpenicillin } \\
\text { and cimetidine }\end{array}$ & - & - & - & - & $\mathrm{N}$ & - \\
\hline
\end{tabular}

$\mathrm{Y}=$ effect seen, $\mathrm{N}=$ no effect seen, $-=$ not investigated.

DMSO showed significant inhibitory effects on $t_{1 / 2}$ in all of the breath tests at the positive control dose $(3520 \mathrm{mg} / \mathrm{kg})$, suggesting inhibition of metabolism by CYP3A, CYP2E1, and the variety of enzymes that metabolise aminopyrine (CYP 2C11, 2C12, 2B1 and 2B2). The positive control dose of DMSO in the renal experiments $\left(4400 \mathrm{mg} / \mathrm{kg}\right.$ ) severely delayed ${ }^{99 \mathrm{~m}}$ Tc-DTPA elimination from the kidneys, suggesting that it could affect the renal elimination of drugs. Additionally, dosing with $2200 \mathrm{mg} / \mathrm{kg}$ DMSO for 2 days substantially increased plasma $\alpha_{1}$-AGP levels, which could potentially affect the pharmacokinetics of drugs which bind to this 
protein. These results demonstrate the profound effects on drug disposition that high doses of DMSO can have, and demonstrate that use of such high doses of this excipient should clearly be avoided.

The differences between the effects seen with high dose DMSO and the lower doses commonly used in pre-clinical studies highlight the importance of considering the dose of an excipient when considering the potential pharmacokinetic effects of a formulation. In contrast to high dose DMSO, no effects on metabolism were apparent at the lower doses of DMSO commonly used in pre-clinical studies (220 and $440 \mathrm{mg} / \mathrm{kg}$ ), suggesting that such doses would not affect the pharmacokinetics of drugs which are predominantly eliminated by metabolism. However it is important to bear in mind that these results were obtained after a single acute dose of DMSO; it is possible that multiple administrations of these low doses could have significant effects on metabolism. The results of the ${ }^{99 \mathrm{~m}}$ Tc-DTPA studies in Chapter 6 suggest that $393 \mathrm{mg} / \mathrm{kg}$ DMSO can increase GFR. This suggests that even low doses of DMSO are unsuitable for use in formulations for compounds which undergo a substantial proportion of renal elimination. The lack of effect of DMSO on P-Gp in the in vitro studies described in Chapter 4 suggests that this excipient would be suitable for use in formulations for $P-G p$ substrates, as it would be unlikely to affect their pharmacokinetics 
by P-Gp inhibition. Nor did low dose DMSO $(440 \mathrm{mg} / \mathrm{kg}$ ) have any effect on plasma levels of $\alpha_{1}$-AGP, suggesting that low doses of this co-solvent would not increase protein binding due to increased levels of this protein.

PG, at a dose commonly used in pre-clinical studies $(829 \mathrm{mg} / \mathrm{kg})$, significantly inhibited NDMA clearance in the breath tests, but had no effects on aminopyrine or erythromycin clearance, suggesting specific inhibition of CYP2E1. It therefore appears prudent to avoid use of PG in formulations for drugs which are metabolised by this isoform of CYP450. However, the same dose of PG showed no significant effects on renal elimination in the ${ }^{99 \mathrm{~m}}$ Tc-DTPA studies, suggesting that it is suitable for use in formulating drugs which mainly undergo renal elimination. In the $\alpha_{1}$-AGP experiments, $829 \mathrm{mg} / \mathrm{kg}$ PG only increased plasma $\alpha_{1}$-AGP levels in one out of nine animals. It appears that this increase may not have been an effect of PG, unless the incidence of this effect is very low, and suggests that the co-solvent is unlikely to affect drug pharmacokinetics by increasing levels of this protein. PG had a small but significant inhibitory effect on P-Gp in vitro (Chapter 4), but this was not reproducible, and appeared to correspond to a small but non-significant increase in general permeability of the cell monolayer. It therefore appears that PG would not significantly 
affect the pharmacokinetics of drugs which are P-Gp substrates, suggesting that it is suitable for use in their formulation.

Solutol HS15 inhibited P-Gp in vitro (Chapter 4), and also inhibited biliary elimination by MRP2 and probably also P-Gp in the in vivo ${ }^{99 \mathrm{~m}} \mathrm{Tc}-\mathrm{MIBI}$ and ${ }^{99 \mathrm{~m}} \mathrm{Tc}-\mathrm{HIDA}$ experiments. It therefore seems that Solutol has the potential to substantially affect drug pharmacokinetics by inhibition of P-Gp and MRP, and so should be avoided in formulations for drugs which are substrates for either of these transporters. However, this excipient did not significantly affect active renal secretion due to OATs or OCTs, as demonstrated by its lack of effect on the pharmacokinetic profiles of benzylpenicillin and cimetidine. Solutol therefore appears unlikely to affect renal elimination by this mechanism. In contrast, Solutol did significantly affect the renal elimination of ${ }^{99 \mathrm{~m}} \mathrm{Tc}$-DTPA in some rats, delaying elimination of ${ }^{99 \mathrm{~m}} \mathrm{Tc}$-DTPA from the kidney without affecting its uptake (i.e. GFR). While the manner in which this would impact upon drug pharmacokinetics is unclear, it does highlight the potential of Solutol to interfere with renal elimination, suggesting that it may be unsuitable for use in formulating drugs which undergo substantial elimination by this route. Solutol did not significantly affect the clearance of erythromycin or aminopyrine in the breath tests, suggesting that it did not inhibit metabolism by CYP3A or the variety of enzymes that metabolise aminopyrine (CYP 
$2 \mathrm{C} 11,2 \mathrm{C} 12,2 \mathrm{~B} 1$ and $2 \mathrm{~B} 2$ ). It therefore appears that this excipient may be suitable for use in formulations for compounds predominantly eliminated by these enzymes. Other authors have reported inhibition of the clearance of CYP3A substrates by Solutol in vitro (Bravo Gonzalez et al., 2001; Bittner et al., 2003; Bravo Gonzalez et al., 2004b). Inhibition of metabolism by CYP3A was proposed as the mechanism by which the excipient altered colchicine pharmacokinetics in vivo (Bittner et al., 2003). However, as discussed in Chapter 5, inhibition of P-Gp has similar effects on colchicine pharmacokinetics to those reported by Bittner et al. (2003) (Desrayaud et al., 1997), and may be at least partially responsible for the effects seen with Solutol in their study. It may therefore be wise to use Solutol with caution in formulations for CYP3A substrates until this issue has been clarified. Two $100 \mathrm{mg} / \mathrm{kg}$ doses of Solutol did not affect plasma levels of $\alpha_{1}$-AGP, suggesting that increased protein binding due to increased levels of this protein is not likely to be a mechanism by which Solutol alters drug disposition.

Tween 80 significantly inhibited P-Gp in vitro, and significantly inhibited biliary elimination due to $\mathrm{P}-\mathrm{Gp}$, but not $\mathrm{MRP}$, in the in vivo ${ }^{99 \mathrm{~m}} \mathrm{Tc}-\mathrm{MIBI}$ and ${ }^{99 \mathrm{~m}} \mathrm{Tc}-\mathrm{HIDA}$ studies. This suggests that Tween is unsuitable for use in formulating P-Gp substrates, as it has the potential to affect their disposition by inhibition of P-Gp. 
In the renal studies, Tween showed similar effects to Solutol, impairing elimination of ${ }^{99 \mathrm{~m}}$ Tc-DTPA from the kidneys without affecting GFR. Again, the potential impact of this effect on drug pharmacokinetics is unclear, but it does demonstrate the potential of this excipient to affect renal elimination, suggesting that its use should be avoided in formulations for drugs mainly eliminated by the kidneys. Effects of Tween 80 on metabolism were not investigated in the present studies due to time and resource limitations. However, the literature does contain in vitro evidence of enzyme inhibition by Tween. Mountfield et al. (2000) reported that Tween 80 inhibited human CDNA expressed CYP3A4 at concentrations of $0.005 \%$ and above. Bravo Gonzales et al. (2004b) reported that Tween 80 reduced the clearance of the CYP3A substrate midazolam in rat liver microsomes at concentrations of $0.003 \%$ and above, and in hepatocytes at $0.03 \%$ and above. It would therefore be advisable to avoid the use of Tween 80 in formulations for CYP3A substrates, until it can be determined whether Tween can also inhibit this enzyme in vivo. Two $85 \mathrm{mg} / \mathrm{kg}$ doses of Tween 80 did not increase plasma $\alpha_{1}$-AGP levels, suggesting that the excipient is unlikely to affect drug protein binding by increasing levels of this protein.

Time limitations prevented all of the excipients of interest from being studied in each of the chapters. Thus, PEG and HP- $\beta-C D$ were 
each studied in only one chapter, where literature evidence suggested that they might be likely to show effects. It is not possible to make detailed recommendations for minimising the likelihood of these excipients affecting drug pharmacokinetics based on the present studies. PEG 400 had a small inhibitory effect on PGp at $1 \%$ in the in vitro studies, however this was lost at $0.1 \%$. Although this excipient could therefore theoretically affect the disposition of P-Gp substrates, this seems unlikely to occur at concentrations which would be found after in vivo dosing, although it would be preferable to use an excipient which has no reported effects on P-Gp for formulating P-Gp substrates if possible. Two doses of $360 \mathrm{mg} / \mathrm{kg} \mathrm{HP-} \beta-C D$ did not increase plasma $\alpha_{1}$-AGP levels, suggesting that this excipient is unlikely to affect drug protein binding by increasing levels of this protein.

The present study focussed on effects seen after intravenous and intraperitoneal administration of co-solvents and excipients, to reflect current practice when carrying out early pharmacokinetic studies. However, it is interesting to note that Solutol has been reported to alter the pharmacokinetics of drugs dosed intravenously, after oral dosing of the excipient. Bittner et al. (2005) reported that orally administered Solutol increased $\mathrm{AUC}_{0-\infty}$ $C_{\max }$, and $A_{e}$, and reduced clearance of intravenously administered colchicine in rats. These changes parallel those seen after 
intravenous dosing of Solutol and colchicine, but are smaller in magnitude, and suggest that Solutol or one of its metabolites was absorbed orally in order to exert these effects (Bittner et al., 2005). This highlights the need to consider excipients administered by all routes when considering potential effects on pharmacokinetics, not just those co-formulated with the drug. However, this does not appear to be the case for Tween 80. Zhang et al. (2003) reported that orally administered Tween did not affect the pharmacokinetics of intravenously administered digoxin, although digoxin clearance was reduced and AUC increased when both drug and excipient were dosed intravenously.

As well as affecting the pharmacokinetic profile, the effects of excipients on drug disposition described above also have the potential to affect the toxicological profile of drugs. Inhibition of metabolism by a particular isoform of CYP450, as demonstrated in the breath tests, could have several possible effects on a drug's toxicological profile. Inhibition of the isoform of CYP450 responsible for the major route of metabolism of the drug could increase the metabolism of the drug by other enzymes, thus potentially increasing production of a toxic metabolite. Conversely, an excipient could also reduce production of a toxic metabolite by inhibiting the enzyme that produces it, thus improving the toxicological profile of the drug. This was demonstrated in vitro for PG by Snawder et al. 
(1993), who reported that PG inhibited acetaminophen metabolism by CYP2E1 but not CYP1A2 in mouse subcellular liver fractions, and suggested that this was the mechanism by which PG protects against acetaminophen hepatotoxicity. Inhibition of drug metabolism by an excipient could also potentially lead to a compensatory increase in renal elimination of the drug, increasing exposure of kidneys to the drug and potentially increasing any renal toxicity. The impaired ${ }^{99 \mathrm{~m}}$ Tc-DTPA elimination from the kidneys seen with $4400 \mathrm{mg} / \mathrm{kg}$ DMSO, and also in some rats after dosing with Solutol and Tween, could also potentially increase renal toxicity of co-dosed drugs, by increasing their residence time in the kidneys. Altered drug disposition due to inhibition of P-Gp or MRP, as seen in the ${ }^{99 \mathrm{~m}}$ Tc-MIBI and ${ }^{99 \mathrm{~m}}$ Tc-HIDA studies, also has the potential to alter a drug's toxicological profile, by altering the exposure of various organs to the drug. The potential impact of excipientinduced alterations in pharmacokinetics on the toxicological profile of drugs is therefore an important area to consider, and represents an interesting area for further study.

The results described in this thesis and in the literature clearly indicate that excipients can significantly affect drug pharmacokinetics. Although it is important to minimise the occurrence of this in studies where such interference is undesirable, such as studies designed to determine a drugs intrinsic 
pharmacokinetics, there is potential to use these effects to purposely alter drug disposition. For example, if the dose of excipient which would completely inhibit a particular transporter or enzyme could be established and safely administered, this technique could be used in pre-clinical studies in a similar way to knockout mice, to investigate the impact of a particular factor on a drug's pharmacokinetic or toxicological profile. However, deliberate alteration of a drug's pharmacokinetic profile with excipients is unlikely to be a suitable strategy for the clinic, where patients may be receiving other drugs, the pharmacokinetics of which may also be altered by the excipient.

\subsection{Further work}

In addition to the specific further work described at the end of each chapter, there are several other areas of interest for further study. The most obvious avenue for future investigation is to determine the effects of a greater number of excipients on various aspects of pharmacokinetics, such that the potential effects of all of the cosolvents or excipients commonly used in pre-clinical studies are known. This would enable formulations for such studies to be designed based on the minimum likelihood of altering drug pharmacokinetics. It would also be useful to clarify the dose or 
exposure thresholds at which the various pharmacokinetic effects of excipients occur, so that this can be taken into account in formulation design. The present studies investigated the effects of excipients after a single acute dose. Investigation of the effects of chronic excipient dosing on drug disposition is another important area for further study, as it is possible that different effects could occur after chronic dosing of excipients, such as enzyme induction. Although investigation into the mechanisms behind excipient effects on pharmacokinetics was beyond the scope of this thesis, such investigations would be useful to perform, to enable these effects to be better understood. As discussed above, the potential impact of excipient-induced alterations in pharmacokinetics on the toxicological profile of drugs would also be an interesting area for further study. 


\section{References}

Aasmundstad, T. A., Lillekjendlie, B., and Morland, J. (1996). Ethanol interference with morphine metabolism in isolated guinea pig hepatocytes. Pharmacol Toxicol 79, 114-119.

Adachi, M., Sampath, J., Lan, L.-B., Sun, D., Hargrove, P., Flatley, R., Tatum, A., Edwards, M. Z., Wezeman, M., Matherly, L., Drake, R., and Schuetz, J. (2002). Expression of MRP4 confers resistance to ganciclovir and compromises bystander cell killing. J Biol Chem 277, 38998-39004.

Adedoyin, A., Aarons, L., Houston, J.B. (1987). Dose-dependent pharmacokinetics of cimetidine in the rat. Xenobiotica 17, 595-604.

Ali, B. H., and Mousa, H. M. (2001). Effect of dimethyl sulfoxide on gentamicin-induced nephrotoxicity in rats. Hum Exp Toxicol 20, 199-203.

Alyautdin, R. N., Tezikov, E. B., Ramge, P., Kharkevich, D. A., Begley, D. J., and Kreuter, J. (1998). Significant entry of tubocurarine into the brain of rats by adsorption to polysorbate 80 -coated polybutylcyanoacrylate nanoparticles: an in situ brain perfusion study. J Microencapsul 15, 67-74.

Arimori, K., and Uekama, K. (1987). Effects of beta- and gammacyclodextrins on the pharmacokinetic behavior of prednisolone after intravenous and intramuscular administrations to rabbits. J Pharmacobiodyn 10, 390-395.

Arnaud, M. J. (1976). Identification, kinetic and quantitative study of [2-14C] and [1-Me-14C] caffeine metabolites in rats urine by chromatographic separations. Biochem Med 16, 67-76.

Arnold, F. J., and Meyerson, L. R. (1990). Radial immunodiffusion assay for rat alpha 1-acid glycoprotein. Pharmacol Biochem Behav 37, 485-491.

Ayrton, A., and Morgan, P. (2001). Role of transport proteins in drug absorption, distribution and excretion. Xenobiotica 31, 469-497.

Azmin, M. N., Stuart, J. F. B., and Florence, A. T. (1985). The distribution and elimination of methotrexate in mouse blood and brain after concurrent administration of polysorbate 80 . Cancer Chemoth Pharm 14, 238-242.

Badary, O. A., Al-Shabanah, O. A., Al-Gharably, N. M., and Elmazar, M. M. A. (1998). Effect of Cremophor EL on the pharmacokinetics, antitumor activity and toxicity of doxorubicin in mice. Anti-Cancer Drug 9, 809-815.

Barbarics, E., Kronauge, J. F., Cohen, D., Davison, A., Jones, A. G., and Croop, J. M. (1998). Characterization of P-glycoprotein transport and inhibition in vivo. Cancer Res 58, 276-282. 
Bardelmeijer, H. A., Ouwehand, M., Malingre, M. M., Schellens, J. H., Beijnen, J. H., and van Tellingen, O. (2002). Entrapment by Cremophor EL decreases the absorption of paclitaxel from the gut. Cancer Chemoth Pharm 49, 119-125.

Bar-Meir, S., Baron, J., Seligson, U., Gottesfeld, F., Levy, R., and Gilat, T. (1982). 99mTc-HIDA cholescintigraphy in DubinJohnson and Rotor syndromes. Radiology 142, 743-746.

BASF (2004). Cremophor grades. www.BASF-pharma.com.

Basit, A. W., Podczeck, F., Newton, J. M., Waddington, W. A., Ell, P. J., and Lacey, L. F. (2002). Influence of polyethylene glycol 400 on the gastrointestinal absorption of ranitidine. Pharm Res 19, 1368-1374.

Bastien, M. C., and Villeneuve, J. P. (1998). Characterization of cytochrome P450 2E1 activity by the [14C]nitrosodimethylamine breath test. Can J Physiol Pharmacol 76, 756-763.

Bastien, M. C., Leblond, F., Pichette, V., and Villeneuve, J. P. (2000). Differential alteration of cytochrome P450 isoenzymes in two experimental models of cirrhosis. Can J Physiol Pharmacol 78, 912-919.

Baumann, P., Tinguely, D., and Schopf, J. (1982). Increase of alpha 1-acid glycoprotein after treatment with amitriptyline. $\mathrm{Br} \mathrm{J}$ Clin Pharmacol 14, 102-103.

Bergholz, H., Erttmann, R.R., Damm, K.H. (1980). Effects of probenecid on plasma/tissue distribution of 14Cbenzylpenicillin in rats. Experientia 36, 333-334.

Besarab, A., Jarrell, B. E., Hirsch, S., Carabasi, R. A., Cressman, M. D., and Green, P. (1987). Use of the isolated perfused kidney model to assess the acute pharmacologic effects of cyclosporine and its vehicle, cremophor EL. Transplantation 44, 195-201.

Bettschart-Wolfensberger, R., Semder, A., Alibhai, H., Demuth, D., Aliabadi, F. S., and Clarke, K. W. (2000). Cardiopulmonary side-effects and pharmacokinetics of an emulsion of propofol (Disoprivan (R)) in comparison to propofol solved in polysorbate 80 in goats. $J$ Vet Med A 47, 341-350.

Bittner, B., Bravo Gonzalez, R. C., Walter, I., and Huwyler, J. (2005). Impact of Oral Administration of the Surface-Active Excipient Solutol HS 15 on the Pharmacokinetics of Intravenously Administered Colchicine. Lett Drug Des Discov 2, 193-195.

Bittner, B., Bravo Gonzalez, R. C., Isel, H., and Flament, C. (2003a). Impact of Solutol HS 15 on the pharmacokinetic behaviour of midazolam upon intravenous administration to male Wistar rats. Eur J Pharm Biopharm 56, 143-146.

Bittner, B., Gonzalez, R. C., Walter, I., Kapps, M., and Huwyler, J. (2003b). Impact of Solutol HS 15 on the pharmacokinetic 
behaviour of colchicine upon intravenous administration to male Wistar rats. Biopharm Drug Dispos 24, 173-181.

Bittner, B., Guenzi, A., Fullhardt, P., Zuercher, G., Gonzalez, R. C., and Mountfield, R. J. (2002). Improvement of the bioavailability of colchicine in rats by co-administration of $D$ alpha-tocopherol polyethylene glycol 1000 succinate and a polyethoxylated derivative of 12 -hydroxy-stearic acid. ArzneiForschung 52, 684-688.

Bittner, B., and Mountfield, R. J. (2002). Intravenous administration of poorly soluble new drug entities in early drug discovery: The potential impact of formulation on pharmacokinetic parameters. Curr Opin Drug Di De 5, 59-71.

Bodd, E., Olsen, H., Gulliksen, M., and Mørland, J. (1985). Ethanol does not increase lethality due to propoxyphene in rats. Alcohol 2, 293-296.

Bogman, K., Erne-Brand, F., Alsenz, J., and Drewe, J. (2003). The role of surfactants in the reversal of active transport mediated by multidrug resistance proteins. J Pharm Sci 92, 1250-1261.

Borst, P., Evers, R., Kool, M., and Wijnholds, J. (1999). The multidrug resistance protein family. . Biochim Biophys Acta 1461, 347-357.

Brandt, I., Brittebo, E. B., Larsson, Y., Lindqvist, N. G., and Ullberg, S. (1982). Selective affinity of dimethyl sulphoxide (DMSO) and 2-amino-4-phenylsulphonylbenzenesulphonamide (NSD 3004) for the large intestinal mucosa of mice. Acta Pharmacol Toxicol (Copenh) 51, 173-176.

Brasseur, N., Ouellet, R., Lewis, K., Potter, W. R., and van Lier, J. E. (1995). Photodynamic activities and skin photosensitivity of the bis(dimethylthexylsiloxy)silicon 2,3-naphthalocyanine in mice. Photochem Photobiol 62, 1058-1065.

Bravo Gonzalez, R. C., Boess, F., Durr, E., Schaub, N., and Bittner, B. (2004a). In vitro investigation on the impact of Solutol HS 15 on the uptake of colchicine into rat hepatocytes. Int $J$ Pharm 279, 27-31.

Bravo Gonzalez, R. C., Huwyler, J., Boess, F., Walter, I., and Bittner, B. (2004b). In vitro investigation on the impact of the surface-active excipients Cremophor EL, Tween 80 and Solutol HS 15 on the metabolism of midazolam. Biopharm Drug Dispos 25, 37-49.

Bravo Gonzalez, R. C., Huwyler, J., Walter, I., Mountfield, R., and Bittner, B. (2002). Improved oral bioavailability of cyclosporin A in male Wistar rats. Comparison of a Solutol HS15 containing self-dispersing formulation and a microsuspension. Int J Pharm 245, 143-151.

Bravo Gonzalez, R. C., Mountfield, R. J., Walter, I., Delobel, F., and Bittner, B. (2001). Impact of Solutol HS 15 on the clearance of midazolam in rat hepatocytes. Drug Met Rev 33, 364. 
Brime, B., Frutos, P., Bringas, P., Nieto, A., Ballesteros, M. P., and Frutos, G. (2003). Comparative pharmacokinetics and safety of a novel lyophilized amphotericin B lecithin-based oil-water microemulsion and amphotericin B deoxycholate in animal models. J Antimicrob Chemother 52, 103-109.

Brink, J. J., and Stein, D. G. (1967). Pemoline levels in brain: enhancement by dimethyl sulfoxide. Science 158, 1479-1480. Burak, Z., Moretti, J. L., Ersoy, O., Sanli, U., Kantar, M., Tamgac, F., and Basdemir, G. (2003). 99mTc-MIBI imaging as a predictor of therapy response in osteosarcoma compared with multidrug resistance-associated protein and P-glycoprotein expression. J NuCl Med 44, 1394-1401.

Campbell, L., Abulrob, A. N., Kandalaft, L. E., Plummer, S., Hollins, A. J., Gibbs, A., and Gumbleton, M. (2003). Constitutive expression of $\mathrm{p}$-glycoprotein in normal lung alveolar epithelium and functionality in primary alveolar epithelial cultures. J Pharmacol Exp Ther 304, 441-452.

Cha, S.H., Sekine, T., Fukushima, J.I., Kanai, Y., Kobayashi, Y., Goya, T., Endou, H. (2001). Identification and characterization of human organic anion transporter 3 expressing predominantly in the kidney. Mol Pharmacol 59, 1277-11286.

Chang, C. S., Huang, W. T., Yang, S. S., Yeh, H. Z., Kao, C. H., and Chen, G. H. (2003). Effect of P-glycoprotein and multidrug resistance associated protein gene expression on Tc-99m MIBI imaging in hepatocellular carcinoma. Nucl Med Biol 30, 111-117.

Chauret, N., Gauthier, A., and Nicoll-Griffith, D. A. (1998). Effect of common organic solvents on in vitro cytochrome P450mediated metabolic activities in human liver microsomes. Drug Metab Dispos 26, 1-4.

Choo, E. F., Leake, B., Wandel, C., Imamura, H., Wood, A. J., Wilkinson, G. R., and Kim, R. B. (2000). Pharmacological inhibition of P-glycoprotein transport enhances the distribution of HIV-1 protease inhibitors into brain and testes. Drug Metab Dispos 28, 655-660.

Christensen, J. M., Suvanakadt, U., Ayres, J. W., and Tavipatana, W. (1985). Ethyl lactate - ethanol - water cosolvent for intravenous theophylline. Res Commun Chem Pathol Pharmacol 50, 147-150.

Cisternino, S., Mercier, C., Bourasset, F., Roux, F., and Scherrmann, J. M. (2004). Expression, up-regulation, and transport activity of the multidrug-resistance protein ABCG2 at the mouse blood-brain barrier. Cancer Res 64, 3296-3301. Colombo, T., Parisi, I., Zucchetti, M., Sessa, C., Goldhirsch, A., and D'Incalci, M. (1999). Pharmacokinetic interactions of 
paclitaxel, docetaxel and their vehicles with doxorubicin. Ann Oncol 10, 391-395.

Coon, J. S., Knudson, W., Clodfelter, K., Lu, B., and Weinstein, R. S. (1991). Solutol HS 15, nontoxic polyoxyethylene esters of 12hydroxystearic acid, reverses multidrug resistance. Cancer Res 51, 897-902.

Cornaire, G., Woodley, J. F., Hermann, P., Cloarec, A., and Houin, G. (2004). Impact of excipients on the absorption of Pglycoprotein substrates in vitro and in vivo. Int J Pharm 278, 119-131.

Cornaire, G., Woodley, J. F., Saivin, S., Legendre, J. Y., Decourt, S., Cloarec, A., and Houin, G. (2000). Effect of polyoxyl 35 castor oil and Polysorbate 80 on the intestinal absorption of digoxin in vitro. Arznei-Forschung 50, 576-579.

Cox, E. H., Knibbe, C. A. J., Koster, V. S., Langemeijer, M. W. E., Tukker, E. E., Lange, R., Kuks, P. F. M., Langemeijer, H. J. M., Lie-A-Huen, L., and Danhof, M. (1998). Influence of different fat emulsion-based intravenous formulations on the pharmacokinetics and pharmacodynamics of propofol. Pharm Res 15, 442-448.

Cummings, J., Forrest, G. J., Cunningham, D., Gilchrist, N. L., and Soukop, M. (1986). Influence of polysorbate-80 (Tween80)and etoposide (VP-16-213) on the pharmacokinetics and urinary excretion of adriamycin and its metabolites in cancer patients. Cancer Chemoth Pharm 17, 80-84.

Dattani, R. G., Harry, F., Hutchings, A. D., and Routledge, P. A. (1999). The effects of acute ethanol intake on isoniazid pharmacokinetics. Brit J Clin Pharmacol 47, 591-592.

De Groof, R. C. (1979). Effect of solvents on the in vitro uptake and distribution of diazepam. Proc West Pharmacol Soc 22, 351354.

Dean, M. E., and Stock, H. (1974). Propylene glycol as a drug solvent in the study of hepatic microsomal enzyme metabolism in the rat. Toxicol Appl Pharmacol 28, 44-52.

Desrayaud, S., Guntz, P., Scherrmann, J. M., and Lemaire, M. (1997). Effect of the P-glycoprotein inhibitor, SDZ PSC 833, on the blood and brain pharmacokinetics of colchicine. Life Sci 61, 153-163.

Dimitrijevic, D., Whitton, P. S., Domin, M., Welham, K., and Florence, A. T. (2001). Increased vigabatrin entry into the brain by polysorbate 80 and sodium caprate. J Pharm Pharmacol 53, 149-154.

Doenicke, A. W., Roizen, M. F., Rau, J., O'Connor, M., Kugler, J., Klotz, U., and Babl, J. (1997). Pharmacokinetics and pharmacodynamics of propofol in a new solvent. Anesth Analg 85, 1399-1403. 
Doenicke, A., Roizen, M. F., Nebauer, A. E., Kugler, A., Hoernecke, R., and Beger-Hintzen, H. (1994). A comparison of two formulations for etomidate, 2-hydroxypropyl-betacyclodextrin (HPCD) and propylene glycol. Anesth Analg 79, 933-939.

Donadio, C., Tramonti, G., Lucchesi, A., Auner, I., and Bianchi, C. (1998). Early glomerular effects of contrast media in rats: evaluation with a simple method. Ren Fail 20, 703-706.

Dutta, S., and Ebling, W. F. (1998a). Formulation-dependent pharmacokinetics and pharmacodynamics of propofol in rats. $J$ Pharm Pharmacol 50, 37-42.

Dutta, S., and Ebling, W. F. (1998b). Formulation-dependent brain and lung distribution kinetics of propofol in rats. Anesthesiology 89, 678-685.

Dutta, S., Matsumoto, Y., and Ebling, W. F. (1997). Propofol pharmacokinetics and pharmacodynamics assessed from a Cremophor EL formulation. J Pharm Sci 86, 967-969.

Dyszlewski, M., Blake, H. M., Dahlheimer, J. L., Pica, C. M., and Piwnica-Worms, D. (2002). Characterization of a novel 99mTc-carbonyl complex as a functional probe of MDR1 Pglycoprotein transport activity. Mol Imaging 1, 24-35.

Effect of ethanol on the pharmacokinetics of penicillin in the rat. Acta Pharmacol Toxicol (Copenh) 53, 200-204.

Egan, T. D., Kern, S. E., Johnson, K. B., and Pace, N. L. (2003). The pharmacokinetics and pharmacodynamics of propofol in a modified cyclodextrin formulation (Captisol) versus propofol in a lipid formulation (Diprivan): an electroencephalographic and hemodynamic study in a porcine model. Anesth Analg 97, 7279.

Ellis, A. G., and Webster, L. K. (1999). Inhibition of paclitaxel elimination in the isolated perfused rat liver by Cremophor EL. Cancer Chemoth Pharm 43, 13-18.

Ellis, A. G., Crinis, N. A., and Webster, L. K. (1996). Inhibition of etoposide elimination in the isolated perfused rat liver by Cremophor EL and Tween 80. Cancer Chemoth Pharm 38, 8187.

Fetterly, G. J., and Straubinger, R. M. (2003). Pharmacokinetics of paclitaxel-containing liposomes in rats. AAPS Pharm Sci 5, article 32.

Flecknell, P., and Orr, H. (2002). Laboratory Animal Anaesthesia, Surgery and Perioperative Care: Interactive course notes. University of Newcastle upon Tyne, Newcastle upon Tyne.

Frijlink, H. W., Franssen, E. J. F., Eissens, A. C., Oosting, R., Lerk, C. F., and Meijer, D. K. F. (1991). The effects of cyclodextrins on the disposition of intravenously injected drugs in the rat. Pharm Res 8, 380-384. 
García, J. J., Bolás, F., and Torrado, J. J. (2003). Bioavailability and efficacy characteristics of two different oral liquid formulations of albendazole. Int J Pharm 250, 351-358.

Gelderblom, H., Verweij, J., Nooter, K., and Sparreboom, A. (2001). Cremophor EL: the drawbacks and advantages of vehicle selection for drug formulation. Eur J Cancer 37, 1590-1598.

Gelderblom, H., Verweij, J., van Zomeren, D. M., Buijs, D., Ouwens, L., Nooter, K., Stoter, G., and Sparreboom, A. (2002). Influence of Cremophor El on the bioavailability of intraperitoneal paclitaxel. Clin Cancer Res 8, 1237-1241. Gerasimov, M. R., Logan, J., Ferrieri, R. A., Muller, R. D., Alexoff, D., and Dewey, S. L. (2002). Effect of vehicle on brain uptake of [11C]toluene. NuCl Med Biol 29, 607-612.

Gole, D. J., and Nagwekar, J. B. (1991). Effects of chronic ethanol ingestion on pharmacokinetics of procainamide in rats. $J$ Pharm Sci 80, 232-238.

Gould, S., and Scott, R. C. (2005). 2-Hydroxypropyl-B-cyclodextrin (HP-B-CD): A toxicology review. Food Chem Toxicol 43, 14511459.

Grauer, G. F., Hull Thrall, M. A., Henre, B. A., and Hjelle, J. J. (1987). Comparison of the effects of ethanol and 4methylpyrazole on the pharmacokinetics and toxicity of ethylene glycol in the dog. Toxicol Lettl 35, 307-314.

Gross, S. M., Reddy, R. V., and Reddy, C. S. (1993). Alteration in the tissue retention of [14C]-caffeine in pregnant mice by dimethylsulfoxide. Nat Toxins 1, 376-380.

Grosse, P. Y., Bressolle, F., Rouanet, P., Joulia, J. M., and Pinguet, F. (1999). Methyl--cyclodextrin and doxorubicin pharmacokinetics and tissue concentrations following bolus injection of these drugs alone or together in the rabbit. Int $J$ Pharm 180, 215-223.

Gruys, E., Toussaint, M. J., Niewold, T. A., and Koopmans, S. J. (2005). Acute phase reaction and acute phase proteins. $J$ Zhejiang Univ Sci B 6, 1045-1056.

Gulyaev, A. E., Gelperina, S. E., Skidan, I. N., Antropov, A. S., Kivman, G. Y., and Kreuter, J. (1999). Significant transport of doxorubicin into the brain with polysorbate 80 -coated nanoparticles. Pharm Res 16, 1564-1569.

Gumbleton, M., and Audus, K. L. (2001). Progress and limitations in the use of in vitro cell cultures to serve as a permeability screen for the blood-brain barrier. J Pharm Sci 90, 16811698.

Gumbleton, M., Nicholls, P. J., and Taylor, G. (1990). Differential influence of laboratory anaesthetic regimens upon renal and hepatosplanchnic haemodynamics in the rat. $J$ Pharm Pharmacol 42, 693-697. 
Gunn, I. R., and Acomb, C. (1986). High plasma osmolality following intravenous dimethylsulfoxide in the treatment of postoperative hemiplegia. J Neurol Neurosur Ps 49, 961-962.

Guo, J. X., Ping, Q. N., and Chen, Y. (2001). Pharmacokinetic behavior of cyclosporin $A$ in rabbits by oral administration of lecithin vesicle and sandimmun neoral. Int J Pharm 216, 1721.

Harrison, S. D., Cusic, A. M., and McAfee, S. M. (1981). Tween 80 increases plasma adriamycin concentrations in mice by a apparent reduction of plasma volume. Eur J Cancer 17, 387389.

Hasegawa, M., Kusuhara, H., Endou, H., and Sugiyama, Y. (2003). Contribution of organic anion transporters to the renal uptake of anionic compounds and nucleoside derivatives in rat. $J$ Pharmacol Exp Ther 305, 1087-1097.

Hayman, M., Seidl, E. C., Ali, M., and Malik, K. (2003). Acute tubular necrosis associated with propylene glycol from concomitant administration of intravenous lorazepam and trimethoprim-sulfamethoxazole. Pharmacotherapy 23, 11901194.

He, Z. G., Li, Y. S., Zhang, T. H., Tang, X., Zhao, C., and Zhang, R. $H$. (2004). Effects of 2-hydroxypropyl-beta-cyclodextrin on pharmacokinetics of digoxin in rabbits and humans.

Pharmazie 59, 200-202.

Henderson, G.I, Speeg, K.V.Jr. , Roberts, R.K., Perez, A., Schenker, S. (1988). Effect of aging on hepatic elimination of cimetidine and subsequent interaction of aging and cimetidine on aminopyrine metabolism. Biochem Pharmacol 37, 2667-2673.

Hendrikse, N. H., Franssen, E. J., van der Graaf, W. T., Meijer, C., Piers, D. A., Vaalburg, W., and de Vries, E. G. (1998). 99mTcsestamibi is a substrate for P-glycoprotein and the multidrug resistance-associated protein. $\mathrm{Br} J$ Cancer 77, 353-358.

Hendrikse, N. H., Kuipers, F., Meijer, C., Havinga, R., Bijleveld, C. M., van der Graaf, W. T., Vaalburg, W., and de Vries, E. G. (2004). In vivo imaging of hepatobiliary transport function mediated by multidrug resistance associated protein and $\mathrm{P}$ glycoprotein. Cancer Chemother Pharmacol. 54, 131-138.

Herve, F., d'Athis, P., Tremblay, D., Tillement, J. P., and Barre, J. (2003). Glycosylation study of the major genetic variants of human alpha1-acid glycoprotein and of their pharmacokinetics in the rat. J Chromatogr B Analyt Technol Biomed Life Sci 798, 283-294.

Hosokawa, T., Yamauchi, M., Yamamoto, Y., Iwata, K., Mochizuki, H., and Kato, Y. (2002). Role of the lipid emulsion on an injectable formulation of lipophilic KW-3902, a newly synthesized adenosine A1-receptor antagonist. Biol Pharm Bull 25, 492-498. 
Hoyo-Vadillo, C., Mandema, J. W., and Danhof , M. (1995). Pharmacodynamic interaction between midazolam and a low dose of ethanol in vivo. Life Sci 57, 325-333.

Hugger, E. D., Novak, B. L., Burton, P. S., Audus, K. L., and Borchardt, R. T. (2002). A comparison of commonly used polyethoxylated pharmaceutical excipients on their ability to inhibit P-glycoprotein activity in vitro. J Pharm Sci 91, 19912002.

Imamura, H., Maruyama, T., Okabe, H., Shimada, H., and Otagiri, M. (1994). A Simple and Rapid Fluorometric Determination Method of Alpha 1-Acid Glycoprotein in Serum Using Quinaldine Red. Pharmaceut Res 11, 566-570.

Imaoka, S., Inoue, K., and Funae, Y. (1988). Aminopyrine metabolism by multiple forms of cytochrome P-450 from rat liver microsomes: simultaneous quantitation of four aminopyrine metabolites by high-performance liquid chromatography. Arch Biochem Biophys 265, 159-170.

Inui, K. I., Masuda, S., and Saito, H. (2000). Cellular and molecular aspects of drug transport in the kidney. Kidney Int 58, 944958.

Ishikawa, M., Yoshii, H., and Furuta, T. (2005). Interaction of modified cyclodextrins with cytochrome P-450. Biosci Biotechnol Biochem 69, 246-248.

Israili, Z. H., and Dayton, P. G. (2001). Human alpha-1glycoprotein and its interactions with drugs Drug Metab Rev 33, 161-235.

Issar, M., Singh, S. K., Mishra, B., and Gupta, R. C. (2003). Pharmacokinetics, in-situ absorption and protein binding studies of a new neuroleptic agent centbutindole in rats. Eur J Pharm Sci 19, 105-113.

Ivanovic Matic, S., Dinic, S., Mihailovic, M., Grigorov, I., Bogojevic, D., and Poznanovic, G. (2004). Acute-phase protein expression in DMSO-intoxicated rats. Toxicol Lett 147, 153159.

Iwen, P. C., and Miller, N. G. (1986). Enhancement of ketoconazole penetration across the blood-brain barrier of mice by dimethyl sulfoxide. Antimicrob Agents Chemother 30, 617-618.

Jariyawat, S., Sekine, T., Takeda, M., Apiwattanakul, N., Kanai, Y., Sophasan, S., and Endou, H. (1999). The interaction and transport of beta-lactam antibiotics with the cloned rat renal organic anion transporter 1. J Pharmacol Exp Ther 290, 672677.

Ji, L., Masuda, S., Saito, H., Inui, K. (2002). Down-regulation of rat organic cation transporter rOCT2 by $5 / 6$ nephrectomy. Kidney Int 62, 514-524.

Jin, M., Shimada, T., Yokogawa, K., Nomura, M., Mizuhara, Y., Furukawa, Ishizaki, J., and Miyamoto, K. I. (2005). 
Cremophor EL releases cyclosporin A adsorbed on blood cells and blood vessels, and increases apparent plasma concentration of cyclosporin A. Int J Pharm 293, 137-144.

Johnson, B. M., Charman, W. N., and Porter, C. J. H. (2002). An in vitro examination of the impact of polyethylene glycol 400, Pluronic P85, and vitamin E d-a-tocopheryl polyethylene glycol 1000 succinate on P-glycoprotein efflux and enterocyte-based metabolism in excised rat intestine. AAPS Pharm Sci 4, 40.

Kamath, A. V., Chang, M., Lee, F. Y., Zhang, Y., and Marathe, P. H. (2005). Preclinical pharmacokinetics and oral bioavailability of BMS-310705, a novel epothilone B analog. Cancer Chemoth Pharm 56, 145-153.

Kambam, J. R., Franks, J. J., Janicki, P. K., Mets, B., vd Watt, M., and Hickman, R. (1994). Alcohol pretreatment alters the metabolic pattern and accelerates cocaine metabolism in pigs. Drug Alcohol Depend 36, 9-13.

Karyekar, C. S., Eddington, N. D., Garimella, T. S., Gubbins, P. O., and Dowling, T. C. (2003). Evaluation of P-glycoproteinmediated renal drug interactions in an MDR1-MDCK model. Pharmacotherapy 23, 436-442.

Kassell, N. F., Sprowell, J. A., Boarini, D. J., and Olin, J. J. (1983). Effect of dimethyl sulfoxide on the cerebral and systemic circulations of the dog. Neurosurgery 12, 24-28.

Kawalec, J. C., and Andrews, A. W. (1980). The effect of solvents on drug metabolism in vitro. Drug Metab Dispos 8, 380-384.

Kemper, E. M., van Zandbergen, A. E., Cleypool, C., Mos, H. A., Boogerd, W., Beijnen, J. H., and van Tellingen, O. (2003). Increased penetration of paclitaxel into the brain by inhibition of P-Glycoprotein. Clin Cancer Res 9, 2849-2855.

Kerl, M. E., and Cook, C. R. (2005). Glomerular filtration rate and renal scintigraphy. Clin Tech Small An P 20, 31-38.

Kim, S. C., Kim, D. W., Shim, Y. H., Bang, J. S., Oh, H. S., Kim, S. W., and Seo, M. H. (2001). In vivo evaluation of polymeric micellar paclitaxel formulation: toxicity and efficacy. $J$ Control Release 72, 191-202.

Kim, S. J., Choi, H. K., and Lee, Y. B. (2002). Pharmacokinetic and pharmacodynamic evaluation of cyclosporin A O/W-emulsion in rats. Int J Pharm 249, 149-156.

Kim, Y. K., Lee, S. H., Goldinger, J. M., and Hong, S. K. (1986). Effect of ethanol on organic ion transport in rabbit kidney. Toxicol Appl Pharmacol 86, 411-420.

Kim, Y. S., Cho, S. W., Kim, I. H., Wang, H. I., Jin, Y. M., and Park, C. $H$. (1998). Clinical validation of the influence of $P$ glycoprotein on $99 \mathrm{mTc}$-MIBI uptake in $99 \mathrm{mTC}$-MIBI SPECT in hepatocellular carcinoma. J Hepatol 28, 89.

Knibbe, C. A. J., Aarts, L. P. H. J., Kuks, P. F. M., Voortman, H. J., Lie-A-Huen, L., Bras, L. J., and Danhof, M. (2000). 
Pharmacokinetics and pharmacodynamics of propofol $6 \%$ SAZN versus propofol $1 \%$ SAZN and Diprivan-10 for shortterm sedation following coronary artery bypass surgery. Eur $J$ Clin Pharmacol 56, 89-95.

Kontir, D. M., Glance, C. A., Clolby, H. D., and Miles, P. R. (1986). Effects of organic-solvent vehicles on benzo(a)pyrene metabolism in rabbit lung microsomes. Biochem Pharmacol 35, 2569-2575.

Kopecky, R. T., McAfee, J. G., Thomas, F. D., Anderson, G. H. J., Hellwig, B., Roskopf, M., and Patchin, D. (1990). Enalaprilatenhanced renography in a rat model of renovascular hypertension. $3 \mathrm{NuCl}$ Med 31, 501-507.

Kotake, A. N., Schreider, B. D., and Latts, J. R. (1982). The in vivo measurement of expired $14 \mathrm{CO} 2$ derived from the $\mathrm{N}$ demethylation of aminopyrine as a reflection of the in vitro hepatic cytochrome P-450 drug-metabolism activity in rats. Drug Metab Dispos 10, 251-258.

Kourtopoulos, H., Bolander, H., Holm, S. E., and Wahlstrom, G. (1983). Effect of ethanol on the pharmacokinetics of penicillin in the rat. Acta Pharmacol Toxicol (Copenh) 53, 200-204.

Kourtopoulos, H., Bolander, H., Holm, S.E., Wahlstrom, G. (1983).

Kremer, J. M. H., Wilting, J., and Janssen, L. H. M. (1988). Drug binding to human alpha-1-acid glycoprotein in health and disease. Pharmacol Rev 40, 1-47.

Kreuter, J. (2004). Influence of the surface properties on nanoparticle-mediated transport of drugs to the brain. $J$ Nanosci Nanotechnol 4, 484-488.

Kreuter, J., Alyautdin, R. N., Kharkevich, D. A., and Ivanov, A. A. (1995). Passage of peptides through the blood-brain barrier with colloidal polymer particles (nanoparticles). Brain Res 674, 171-174.

Kunishio, K., Morisaki, K., Matsumoto, Y., Nagao, S., and Nishiyama, Y. (2003). Technetium-99m sestamibi single photon emission computed tomography findings correlated with P-glycoprotein expression, encoded by the multidrug resistance gene-1 messenger ribonucleic acid, in intracranial meningiomas. Neurol Med Chir (Tokyo). 43, 573-580.

Kurihara, A., Shibayama, Y., Mizota, A., Yasuno, A., Ikeda, M., and Hisaoka, M. (1996). Pharmacokinetics of highly lipophilic antitumor agent palmitoyl rhizoxin incorporated in lipid emulsions in rats. Biol Pharm Bull 19, 252-258.

Kurlansky, P. A., Sadeghi, A. M., Michler, R. E., Coppey, L. J., Re, L. P., Thomas, W. G., Smith, C. R., Reemtsma, K., and Rose, E. A. (1986). Role of the carrier solution in cyclosporine pharmacokinetics in the baboon. $J$ Heart Transplant 5, 312316. 
Kurnik, D., Wood, A. J., and Wilkinson, G. R. (2006). The erythromycin breath test reflects $\mathrm{P}$-glycoprotein function independently of cytochrome P450 3A activity. Clin Pharmacol Ther 80, 228-234.

Kusuhara, H., Sekine, T., Utsunomiya-Tate, N., Tsuda, M., Kojima, R., Cha, S.H., Sugiyama, Y., Kanai, Y., Endou, H. (1999). Molecular cloning and characterization of a new multispecific organic anion transporter from rat brain. J Biol Chem 274, $13675-13680$.

Laine, G. A., Hossain, S. M., Solis, R. T., and Adams, S. C. (1995). Polyethylene glycol nephrotoxicity secondary to prolonged high-dose intravenous lorazepam. Ann Pharmacother 29, 1110-1114.

Leblond, F. A., Giroux, L., Villeneuve, J. P., and Pichette, V. (2000). Decreased in vivo metabolism of drugs in chronic renal failure. Drug Metab Dispos 28, 1317-1320.

Lee, D. W., Lim, H. B., Moon, J. Y., and Park, K. H. (1998). In vitro enhancement of microsomal cytochrome P450-dependent monooxygenases by organic solvents in rat liver. $J$ Biochem Mol Biol 31, 391-398.

Lee, P.S., Han, J.Y., Song, T.W., Sung, J.H., Kwon, O.S., Song, S., Chung, Y.B. (2006). Physicochemical characteristics and bioavailability of a novel intestinal metabolite of ginseng saponin (IH901) complexed with beta-cyclodextrin. Int J Pharm 316, 29-36.

Leyssac, P. P., and Christensen, P. (1995). [51Cr]EDTA for measuring total and single nephron glomerular filtration rate in the rat. Acta Physiol Scand 153, 271-277.

Lin, J. H. (2003). Drug-drug interaction mediated by inhibition and induction of P-glycoprotein. Adv Drug Deliv Rev 55, 53-81.

Litman, T., Brangi, M., Hudson, E., Fetsch, P., Abati, A., Ross, D. D., Miyake, K., Resau, J. H., and Bates, S. E. (2000). The multidrug-resistant phenotype associated with overexpression of the new ABC half-transporter, MXR (ABCG2). J Cell SCi 113, 2011-2021.

Liu, J., Qiu, L., Gao, J., and Jin, Y. (2006). Preparation, characterization and in vivo evaluation of formulation of baicalein with hydroxypropyl-beta-cyclodextrin. Int J Pharm 312, 137-143.

Loos, W. J., Baker, S. D., Verweij, J., Boonstra, J. G., and Sparreboom, A. (2003). Clinical pharmacokinetics of unbound docetaxel: role of polysorbate 80 and serum proteins. Clin Pharmacol Ther 74, 364-371.

Loos, W. J., Szebeni, J., ten Tije, A. J., Verweij, J., van Zomeren, D. M., Chung, K. N., Nooter, K., Stoter, G., and Sparreboom, A. (2002). Preclinical evaluation of alternative pharmaceutical 
delivery vehicles for paclitaxel. Anti-Cancer Drug 13, 767775.

Loscher, W., Honack, D., Richter, A., Schulz, H. U., Schurer, M., Dusing, R., and Brewster, M. E. (1995). New injectable aqueous carbamazepine solution through complexing with 2hydroxypropyl-beta-cyclodextrin - tolerability and pharmacolkinetics after intravenous injection in comparison to a glycofurol-based formulation. Epilepsia 36, 255-261.

Lote, C. J. (2000a). The Proximal Tubule. In Principles of Renal Physiology, pp. 53-69. Kluwer Academic, Dordrecht.

Lote, C. J. (2000b). Renal Blood Flow and Glomerular Filtration Rate. In Principles of Renal Physiology, pp. 86-95. Kluwer Academic, Dordrecht.

Malingre, M. M., Schellens, J. H., van Tellingen, O., Ouwehand, M., Bardelmeijer, H. A., Rosing, H., Koopman, F. J., Schot, M. E., Ten Bokkel Huinink, W. W., and Beijnen, J. H. (2001). The cosolvent Cremophor EL limits absorption of orally administered paclitaxel in cancer patients. $\mathrm{Br} J$ Cancer 85, 1472-1477.

Martin-Facklam, M., Burhenne, J., Ding, R., Fricker, R., Mikus, G., Walter-Sack, I., and Haefeli, W. E. (2002). Dose-dependent increase of saquinavir bioavailability by the pharmaceutic aid cremophor EL. Br J Clin Pharmacol 53, 576-581.

Matsushima, H., Watanabe, T., and Higuchi, S. (2000). Effect of alpha(1)-acid glycoprotein on the pharmacokinetics of tamsulosin in rats treated with turpentine oil. J Pharm Sci 89, 490-498.

Mayer, U., Wagenaar, E., Dorobek, B., Beijnen, J. H., Borst, P., and Schinkel, A. H. (1997). Full blockade of intestinal Pglycoprotein and extensive inhibition of blood-brain barrier Pglycoprotein by oral treatment of mice with PSC833. J Clin Invest 100, 2430-2436.

McNamara, P.J., Burgio, D., Yoo, S.D. (1992). Pharmacokinetics of cimetidine during lactation: species differences in cimetidine transport into rat and rabbit milk. J Pharmacol Exp Ther 261, 918-923.

Menicagli, S., Longo, V., Mazzaccaro, A., and Gervasi, P. G. (1994). Microsomal metabolism of $\mathrm{N}, \mathrm{N}$-diethylacetamide and $\mathrm{N}, \mathrm{N}-$ dimethylacetamide and their effects on drug-metabolizing enzymes of rat liver. Biochem Pharmacol 48, 717-726.

Mets, B., Kamban, J. R., Janicki, P. K., Franks, J., Neveling, U., and Hickman, R. (1996). Alcohol pretreatment does not affect bupivacaine pharmacokinetics in the pig. Pharmacol Res 33, 291-295.

Mico, B. A., Swagzdis, J. E., Hu, H. S., Keefer, L. K., Oldfield, N. F., and Garland, W. A. (1985). Low-dose in vivo pharmacokinetic and deuterium isotope effect studies of $\mathrm{N}$ -

nitrosodimethylamine in rats. Cancer Res 45, 6280-6285. 
Millward, M. J., Webster, L. K., Rischin, D., Ellis, A. G., Linahan, B. M., Maisano, R., Toner, G. C., and Stokes, K. H. (1998). Randomised, cross-over trial to determine the effect of Cremophor on the pharmacokinetics and pharmacodynamics of carboplatin-based therapy. Ann Oncol 9, 499.

Morshed, K. M., Desjeux, J. F., Nagpaul, J. P., Majumdar, S., and Amma, M. K. P. (1991). The effect of propane-diols on the intestinal uptake of nutrients and brush-border membrane enzymes in the rat. Biochem Med Metab B 45, 161-170.

Morshed, K. M., Jain, S. K., and McMartin, K. E. (1994). Acute toxicity of propylene glycol: an assessment using cultured proximal tubule cells of human origin. Fundam Appl Toxicol 23, 38-43.

Motohashi, H., Uwai,Y., Hiramoto', K., Okuda, M., Inui, K. (2004). Different transport properties between famotidine and cimetidine by human renal organic ion transporters (SLC22A) Eur J Pharmacol 503, 25-30.

Mountfield, R. J., Senepin, S., Schleimer, M., Walter, I., and Bittner, B. (2000). Potential inhibitory effects of formulation ingredients on intestinal cytochrome P450. Int J Pharm 211, 89-92.

Muller, B. W., and Albers, E. (1991). Effect of hydrotropic substances on the complexation of sparingly soluble drugs with cyclodextrin derivatives and the influence of cyclodextrin complexation on the pharmacokinetics of the drugs. J Pharm Sci 80, 599-604.

Murai-Kuyisha, M., Okada, S., Kimura, T., and Hasegawa, R. (1993). Effects of turpentine oil pretreatment on beta-blocker pharmacokinetic parameters in rats. J Pharm Pharmacol 45, 836-838.

Muther, R. S., and Bennett, W. M. (1980). Effects of dimethyl sulfoxide on renal function in man. JAMA 244, 2081-2083.

Neef, M., Ledermann, M., Saegesser, H., Schneider, V., and Reichen, J. (2006). Low-dose oral rapamycin treatment reduces fibrogenesis, improves liver function, and prolongs survival in rats with established liver cirrhosis. $]$ Hepatol 45, 786-796.

Nema, S., Washkuhn, R. J., and Brendel, R. J. (1997). Excipients and their use in injectable products. J Pharm Sci Technol 51, $166-171$.

Oguma, T., and Levy, G. (1981). Acute effect of ethanol on hepatic first-pass elimination of propoxyphene in rats. $J$ Pharmacol Exp Ther 219, 7-13.

Olsen, H., Aune, H., Lilleasen, P., Gulliksen, M., Bodd, E., and Morland, J. (1983). The effect of ethanol intake on propoxyphene absorption and biotransformation in dogs. Acta Pharmacol Toxicol 53, 21. 
Oshiro, T. T., Texeira, C. F. P., and Oga, S. (1990). Propylene glycol enhances anti-inflammatory effects of phenylbutazone. Gen Pharmacol 21, 131-134.

Overbosch, D., Van Gulpen, C., Hermans, J., and Mattie, H. (1988). The effect of probenecid on the renal tubular excretion of benzylpenicillin. $\mathrm{Br} J$ Clin Pharmacol 25, 51-58.

Park, K. M., and Kim, C. K. (1999). Preparation and evaluation of flurbiprofen-loaded microemulsion for parenteral delivery. Int J Pharm 181, 173-179.

Park, K. M., Lee, M. K., Hwang, K. J., and Kim, C. K. (1999). Phospholipid-based microemulsions of flurbiprofen by the spontaneous emulsification process. Int J Pharm 183, 145154.

Parker, R. B., Gades, N., Mandrell; T., and Laizure, C. (2002). Effect of ethanol on oral cocaine pharmacokinetics.

Pharmacotherapy 22, 246.

Passananti, G. T., Shively, C. A., and Vesell, E. S. (1975). Orally administered dimethyl sulfoxide and its effects on blood concentrations of salicylic acid, sulfanilamide and warfarin. Ann NY Acad Sci 243.

Paul, M., Dueck, M., Kampe, S., Fruendt, H., and Kasper, S. M. (2003). Pharmacological characteristics and side effects of a new galenic formulation of propofol without soyabean oil. Anaesthesia 58, 1056-1062.

Peng, R., Tu, Y. Y., and Yang, C. S. (1982). The induction and competitive inhibition of a high affinity microsomal nitrosodimethylamine demethylase by ethanol. Carcinogenesis 3, 1457-1461.

Pestel, S., Martin, H.J., Maier, G.M., Guth, B. (2006). Effect of commonly used vehicles on gastrointestinal, renal, and liver function in rats. $J$ Pharmacol Toxicol Methods 54, 200-14.

Peters, A. M. (1991). Quantification of renal haemodynamics with radionuclides. Eur J Nucl Med 18, 274-286.

Petersen, J. S., Shalmi, M., Christensen, S., Haugan, K., and Lomholt, N. (1996). Comparison of the renal effects of six sedating agents in rats. Physiol Behav 60, 759-765.

Piafsky, K. M., Borga, O., Odar-Cederlof, I., Johansson, C., and Sjoqvist, F. (1978). Increased plasma protein binding of propranolol and chlorpromazine mediated by disease-induced elevations of plasma alpha1 acid glycoprotein. $N$ Engl J Med 299, 1435-1439.

Piel, G., Evrard, B., Van Hees, T., and Delattre, L. (1999). Comparison of the IV pharmacokinetics in sheep of miconazole-cyclodextrin solutions and a micellar solution. Int J Pharm 180, 41-45.

Piette, M., Evrard, B., Frankenne, F., Chiap, P., Bertholet, P., Castagne, D., Foidart, J.M., Delattre, L., Piel, G. (2006). 
Pharmacokinetic study of a new synthetic MMP inhibitor (Ro 28-2653) after IV and oral administration of cyclodextrin solutions. Eur J Pharm Sci 28, 189-195.

Pitha, J., Gerloczy, A., and Olivi, A. (1994). Parenteral hydroxypropyl cyclodextrins: intravenous and intracerebral administration of lipophiles. J Pharm Sci 83, 833-837.

Piwnica-Worms, D., Chiu, M. L., Budding, M., Kronauge, J. F., Kramer, R. A., and Croop, J. M. (1993). Functional imaging of multidrug-resistant P-glycoprotein with an organotechnetium complex. Cancer Res 53, 977-984.

Preheim, L. C., Olsen, K. M., Yue, M., Snitily, M. U., and Gentry, M. J. (1999). Ethanol feeding does not affect the efficacy or pharmacokinetics of azithromycin, trovafloxacin, or ceftriaxone in a rat model of pneumococcal pneumonia. Alcohol Clin Exp Res 23, 842-849.

Projean, D., Dautrey, S., Vu, H. K., Groblewski, T., Brazier, J. L., and Ducharme, J. (2005). Selective downregulation of hepatic cytochrome P450 expression and activity in a rat model of inflammatory pain. Pharmaceut Res 22, 62-70.

Provencher, S. J., Demers, C., Bastien, M. C., Villeneuve, J. P., and Gascon-Barre, M. (1999). Effect of cyclosporine A on cytochrome P-450-mediated drug metabolism in the partially hepatectomized rat. Drug Metab Dispos 27, 449-455.

Provoost, A. P., and van Aken, M. (1985). Renal adaptation to additional nephrons: a functional study in the three-kidney rat. Renal Physiol 8, 129-135.

Ramzan, I., Yeganeh, M.H. (2002). Pharmacokinetics of H2antagonists during propofol or thiopentone anaesthesia in rats. Ind J Pharm 34, 56-58

Ratnayake, H. J., Hanna, P. E., Anders, M. W., and Duggan, D. E. (1981). In vitro reduction of sulindac by rat hepatic cytosolic enzymes. Drug Metab Dispos 9, 85-87.

Rendic, S., and Di Carlo, F. J. (1997). Human cytochrome P450 enzymes: a status report summarizing their reactions, substrates, inducers, and inhibitors. Drug Metab Rev 29, 413580.

Robertson, E. E., and Rankin, G. O. (2006). Human renal organic anion transporters: characteristics and contributions to drug and drug metabolite excretion. Pharmacol Ther 109, 399-412.

Rubinstein, E., and Lev-El, A. (1980). The effect of dimethyl sulfoxide on tissue distribution of gentamicin. Experientia 36, 92-93.

Sakaeda, T., and Hirano, K. (1995). O/W lipid emulsions for parenteral drug delivery. II. Effect of composition on pharmacokinetics of incorporated drug. J Drug Target 3, 221230. 
Schaad, H. J., Renner, E. L., Wietholtz, H., Arnaud, M. J., and Preisig, R. (1995). Caffeine demethylation measured by breath analysis in experimental liver injury in the rat. $J$ Hepatol 22, 82-87.

Schinkel, A. H., and Jonker, J. W. (2003). Mammalian drug efflux transporters of the ATP binding cassette $(A B C)$ family: an overview. Adv Drug Deliv Rev 55, 3-29.

Schulze, J. D., Peters, E. E., Vickers, A. W., Staton, J. S., Coffin, M. D., Parsons, G. E., and Basit, A. W. (2005). Excipient effects on gastrointestinal transit and drug absorption in beagle dogs. Int J Pharm 300, 67-75.

Schulze, J. D., Waddington, W. A., Eli, P. J., Parsons, G. E., Coffin, M. D., and Basit, A. W. (2003). Concentration-dependent effects of polyethylene glycol 400 on gastrointestinal transit and drug absorption. Pharm Res 20, 1984-1988.

Shono, Y., Nishihara, H., Matsuda, Y., Furukawa, S., Okada, N., Fujita, T., and Yamamoto, A. (2004). Modulation of intestinal P-glycoprotein function by cremophor EL and other surfactants by an in vitro diffusion chamber method using the isolated rat intestinal membranes. J Pharm Sci 93, 877-885.

Snawder, J. E., Benson, R. W., Leakey, J. E., and Roberts, D. W. (1993). The effect of propylene glycol on the P450-dependent metabolism of acetaminophen and other chemicals in subcellular fractions of mouse liver. Life Sci 52, 183-189.

Sokal, R. R., and Rohlf, F. J. (1995). Biometry. W H Freeman and company, New York.

Sparreboom, A., van Tellingen, O., Nooijen, W. J., and Beijnen, J. $\mathrm{H}$. (1996). Nonlinear pharmacokinetics of paclitaxel in mice results from the pharmaceutical vehicle Cremophor EL. Cancer Res 56, 2112-2115.

Sparreboom, A., van Zuylen, L., Brouwer, E., Loos, W. J., de Bruijn, P., Gelderblom, H., Pillay, M., Nooter, K., Stoter, G., and Verweij, J. (1999). Cremophor EL-mediated Alteration of Paclitaxel Distribution in Human Blood: Clinical

Pharmacokinetic Implications. Cancer Res 59, 1454-1457. Sparreboom, A., Verweij, J., van der Burg, M. E., Loos, W. J., Brouwer, E., Vigano, L., Locatelli, A., de Vos, A. I., Nooter, K., Stoter, G., and Gianni, L. (1998). Disposition of Cremophor EL in humans limits the potential for modulation of the multidrug resistance phenotype in vivo. Clin Cancer Res 4, 1937-1942.

Speeg, K. V., Maldonado, A. L., Liaci, J., and Muirhead, D. (1992). Effect of cyclosporine on colchicine secretion by the kidney multidrug transporter studied in vivo. J Pharmacol Exp Ther 261, 50-55.

Stella, V. J., Lee, H. K., and Thompson, D. O. (1995a). The effect of SBE4- $\beta-C D$ on i.m. prednisolone pharmacokinetics and tissue 
damage in rabbits: Comparison to a co-solvent solution and a water-soluble prodrug Int J Pharm 120, 197-204

Stella, V. J., Lee, H. K., and Thompson, D. O. (1995b). The effect of SBE4- $\beta-C D$ on i.v. methylprednisolone pharmacokinetics in rats: Comparison to a co-solvent solution and two watersoluble prodrugs Int J Pharm 120, 189-195

Sugihara, N., Furino, K., Kita, N., Murakami, T., and Yata, N. (1993). The influence of increased plasma-protein binding on the disposition of quinidine in turpentine-treated rats. Biol Pharm Bull 16, 63-67.

Sun, H., Dai, H., Shaik, N., and Elmquist, W. F. (2003). Drug efflux transporters in the CNS. Adv Drug Deliv Rev 55, 83-105.

Sun, J., He, Z. G., Cheng, G., Wang, S. J., Hao, X. H., and Zou, M. J. (2004). Multidrug resistance P-glycoprotein: crucial significance in drug disposition and interaction. Med Sci Monit 10, RA5-14.

Sun, S. S., Hsieh, J. F., Tsai, S. C., Ho, Y. J., K, L. J., and Kao, C. H. (2000). Expression of mediated P-glycoprotein multidrug resistance related to Tc-99m MIBI scintimammography results. Cancer Lett 153, 95-100.

Svelander, L., Erlandsson Harris, H., Lorentzen, J. C., Trollmo, C., Klareskog, L., and Bucht, A. (2004). Oligodeoxynucleotides containing $\mathrm{CpG}$ motifs can induce T cell-dependent arthritis in rats. Arthritis Rheum. 50, 297-304.

Swanson, B. N., Bappana, V. K., Vlasses, P. H., Rotmensch, H. H., and Ferguson, R. K. (1983a). Dimethylsulfoxide inhibits bioactivation of sulindac. J Lab Clin Med 102, 95-101.

Swanson, B. N., Mojaverian, P., and Boppana, V. K. (1983b). Inhibition of sulindac metabolism by dimethylsulfoxide in the rat. J Toxicol Env Health 12, 213-222.

Swanson, B. N., Mojaverian, P., Boppana, V. K., and Dudash, M. R. (1981). Effect of dimethylsulfoxide on sulindac disposition in rats. Drug Metab Dispos 9, 499-502.

Sykes, E., Woodburn, K., Decker, D., and Kessel, D. (1994). Effects of Cremophor EL on distribution of Taxol to serum lipoproteins. $\mathrm{Br} J$ Cancer 70, 401-404.

Tahara, H., Kusuhara, H., Endou, H., Koepsell, H., Imaoka, T., Fuse, E., Sugiyama, Y. (2005). A species difference in the transport activities of $\mathrm{H} 2$ receptor antagonists by rat and human renal organic anion and cation transporters. J Pharmacol Exp Ther 315, 337-345.

Tanaka, E., and Breimer, D. D. (1997). In vivo function tests of hepatic drug-oxidizing capacity in patients with liver disease. $J$ Clin Pharm Ther 22, 237-249.

Tayrouz, Y., Ding, R., Burhenne, J., Riedel, K. D., Weiss, J., HoppeTichy, T., Haefeli, W. E., and Mikus, G. (2003).

Pharmacokinetic and pharmaceutic interaction between 
digoxin and Cremophor RH40. Clin Pharmacol Ther 73, 397405.

ten Tije, A. J., Verweij, J., Loos, W. J., and Sparreboom, A. (2003). Pharmacological effects of formulation vehicles - Implications for cancer chemotherapy. Clin Pharmacokinet 42, 665-685.

Terao, N., and Shen, D. D. (1983). Alterations in serum protein binding and pharmacokinetics of I-propranolol in the rat elicited by the presence of an indwelling venous catheter. $J$ Pharmacol Exp Ther 227, 369-375.

Tibell, A., Larsson, M., and Alvestrand, A. (1993). Dissolving intravenous cyclosporin $A$ in a fat emulsion carrier prevents acute renal side effects in the rat. Transpl Int 6, 69-72.

Tibell, A., Lindholm, A., Sawe, J., Chen, G., and Norrlind, B. (1995). Cyclosporin $A$ in fat emulsion carriers: experimental studies on pharmacokinetics and tissue distribution. Pharmacol Toxicol 76, 115-121.

Tolando, R., Zanovello, A., Ferrara, R., Iley, J. N., and Manno, M. (2001). Inactivation of rat liver cytochrome P450 (P450) by $\mathrm{N}, \mathrm{N}$-dimethylformamide and $\mathrm{N}, \mathrm{N}$-dimethylacetamide. Toxicol Lett 124, 101-111.

Torres-Molina, F., Aristorena, J. C., Garcia-Carbonell, C., Granero, L., Chesa-Jimenez, J., Pla-Delfina, J., and Peris-Ribera, J. E. (1992). Influence of permanent cannulation of the jugular vein on pharmacokinetics of amoxycillin and antipyrine in the rat. Pharm Res 9, 1587-1591.

Tsuji, A., Terasaki, T., Tamai, I., and Takeda, K. (1990). In vivo evidence for carrier-mediated uptake of beta-lactam antibiotics through organic anion transport systems in rat kidney and liver. J Pharmacol Exp Ther 253, 315-320.

Ueo, H., Motohashi, H., Katsura, T., Inui, K. (2005). Human organic anion transporter hOAT3 is a potent transporter of cephalosporin antibiotics, in comparison with hOAT1. Biochem Pharmacol 70, 1104-1113.

Utsunomiya, K., Ballinger, J. R., Piquette-Miller, M., Rauth, A. M., Tang, W., Su, Z. F., and Ichise, M. (2000). Comparison of the accumulation and efflux kinetics of technetium-99m sestamibi and technetium-99m tetrofosmin in an MRP-expressing tumour cell line. Eur J NuCl Med 27, 1786-1792.

Van de Casteele, M., Roskams, T., Van der Elst, I., van Pelt, J. F., Fevery, J., and Nevens, F. (2004). Halofuginone can worsen liver fibrosis in bile duct obstructed rats. Liver Int 24, 502509.

van Tellingen, O., Beijnen, J. H., Verweij, J., Scherrenburg, E. J., Nooijen, W. J., and Sparreboom, A. (1999). Rapid esterasesensitive breakdown of polysorbate 80 and its impact on the plasma pharmacokinetics of docetaxel and metabolites in mice. Clin Cancer Res 5, 2918-2924. 
van Tellingen, O., Huizing, M. T., Nannan Panday, V. R., Schellens, V. H. M., Nooijen, W. J., and Beijnen, J. H. (2000).

Cremophor EL causes (pseudo-) non-linear pharmacokinetics of paclitaxel in patients. $\mathrm{Br} J$ Cancer 81, 330-335.

VandeWiele, C., Everaert, K., VanderEecken, H., VanHaelst P, Simons, M., and Dierckx, R. A. (1997). Differential renal function measured by Tc-99(m)-DTPA and Tc-99(m)-DMSA in a complete unilateral renal obstruction rat model. Nucl Med Commun 18, 1036-1039.

Vora, J., Wu, Z., Montague, M., Penn, M., and Erow, K. (1999). Influence of dosing vehicles on the preclinical pharmacokinetics of phenolic antioxidants. Res Commun Mol Pathol 104, 93-106.

Voulgari, F., Cummins, P., Gardecki, T. I., Beeching, N. J., Stone, P. C., and Stuart, J. (1982). Serum levels of acute phase and cardiac proteins after myocardial infarction, surgery, and infection. Br Heart J 48, 352-356.

Walters, K. M., Mason, W. D., and Badr, M. Z. (1993). Effect of propylene glycol on the disposition of Dramamine in the rabbit. Drug Metab Dispos 21, 305-308.

Walton, K., Dorne, J. L., and Renwick, A. G. (2001). Uncertainty factors for chemical risk assessment: interspecies differences in the in vivo pharmacokinetics and metabolism of human CYP1A2 substrates. Food Chem Toxicol 93, 667-680.

Wandel, C., Kim, R. B., and Stein, C. M. (2003). "Inactive" excipients such as Cremophor can affect in vivo drug disposition. Clin Pharmacol Ther 73, 394-396.

Wang, P. Y., Kaneko, T., Tsukada, H., Nakano, M., and Sato, A. (1997). Dose- and route-dependent alterations in metabolism and toxicity of chemical compounds in ethanol-treated rats: Difference between highly (Chloroform) and poorly (Carbon tetrachloride) metabolized hepatotoxic compounds. Toxicol Appl Pharm 142, 13-21.

Wang, Q., Rager, J. D., Weinstein, K., Kardos, P. S., Dobson, G. L., Li, J., and Hidalgo, I. J. (2005). Evaluation of the MDR-MDCK cell line as a permeability screen for the blood-brain barrier. Int J Pharm 288, 349-359.

Ward, D. S., Norton, J. R., Guivarc'h, P. H., Litman, R. S., and Bailey, P. L. (2002). Pharmacodynamics and pharmacokinetics of propofol in a medium-chain triglyceride emulsion. Anesthesiology 97, 1401-1408.

Watkins, J. B. r., and Sherman, S. E. (1992). Long-term diabetes alters the hepatobiliary clearance of acetaminophen, bilirubin and digoxin. J Pharmacol Exp Ther 260, 1337-1343.

Webster, L. K., Cosson, E. J., Stokes, K. H., and Millward, M. J. (1996). Effect of the paclitaxel vehicle, Cremophor EL, on the 
pharmacokinetics of doxorubicin and doxorubicinol in mice.

Brit J Cancer 73, 522-524.

Westerberg, G., and Wiklund, L. (2005). Beta-cyclodextrin reduces bioavailability of orally administered [3H]benzo[a]pyrene in the rat. J Pharm Sci 94, 114-119.

Woodburn, K., Chang, C. K., Lee, S., Henderson, B., and Kessel, D. (1994). Biodistribution and PDT efficacy of a ketochlorin photosensitizer as a function of the delivery vehicle.

Photochem Photobiol 60, 154-159.

Woodburn, K., Sykes, E., and Kessel, D. (1995). Interactions of

Solutol HS 15 and Cremophor EL with plasma lipoproteins. Int

J Biochem Cell Biol 27, 693-699.

Woodcock, D. M., Linsenmeyer, M. E., Chojnowski, G., Kriegler, A.

B., Nink, V., Webster, L. K., and Sawyer, W. H. (1992).

Reversal of multidrug resistance by surfactants. $\mathrm{Br} J$ Cancer

66, 62-68.

Woolfrey, S. G., Palin, K. J., and Davis, S. S. (1989). The effect of

Miglycol 812 oil on the oral absorption of propranolol in the

rat. J Pharm Pharmacol 41, 579-581.

Wright, S. H. (2005). Role of organic cation transporters in the renal handling of therapeutic agents and xenobiotics. Toxicol Appl Pharmacol 204, 309-319.

Yamaguchi, S., Yachiku, S., Hashimoto, H., Kaneko, S., Nishihara, M., Niibori, D., Shuke, N., and Aburano, T. (2002). Relation between technetium $99 \mathrm{~m}$-methoxyisobutylisonitrile accumulation and multidrug resistance protein in the parathyroid glands. World J Surg 26, 29-34.

Yasaka, W. J., Sasame, H. A., Saul, W., Maling, H. M., and Gilette, J. R. (1978). Mechanisms in potentiation and inhibition of pharmacological actions of hexobarbital and zoxazolamine by glycofurol. Biochem Pharmacol 27, 2851-2858.

Yaucher, N. E., Fish, J. T., Smith, H. W., and Wells, J. A. (2003). Propylene glycol-associated renal toxicity from lorazepam infusion. Pharmacotherapy 23, 1094-1099.

Yokogawa, K., Jin, M., Furui, N., Yamazaki, M., Yoshihara, H., Nomura, M., Furukawa, H., Ishizaki, J., Fushida, S., Miwa, K., and Miyamoto, K. I. (2004). Disposition kinetics of taxanes after intraperitoneal administration in rats and influence of surfactant vehicles. J Pharm Pharmacol 56, 629-634.

Yoo, J. S., Cheung, R. J., Patten, C. J., Wade, D., and Yang, C. S. (1987). Nature of $\mathrm{N}$-nitrosodimethylamine demethylase and its inhibitors. Cancer Res 47, 3378-3383.

Zhang, H., Yao, M., Morrison, R. A., and Chong, S. (2003).

Commonly used surfactant, Tween 80 , improves absorption of P-glycoprotein substrate, digoxin, in rats. Arch Pharm Res 26, 768-772. 
Zhang, Y., Bachmeier, C., and Miller, D. W. (2003). In vitro and in vivo models for assessing drug efflux transporter activity. Adv Drug Deliv Rev 55, 31-51.

Zitting, A., Vainiotalo, S., and Elovaara, E. (1983).

Dimethylsulfoxide induced activation of renal microsomal ethoxycoumarin O-deethylation. In Extrahepatic Drug Metabolism and Chemical Carcinogenesis (J. Rydstrom, J. Montelius, and M. Bengtsson, Eds.), pp. 241-242. Elsevier Science Publishers, The Netherlands. 


\section{Appendix 1:}

\section{Tables of reported excipient effects on pharmacokinetics}




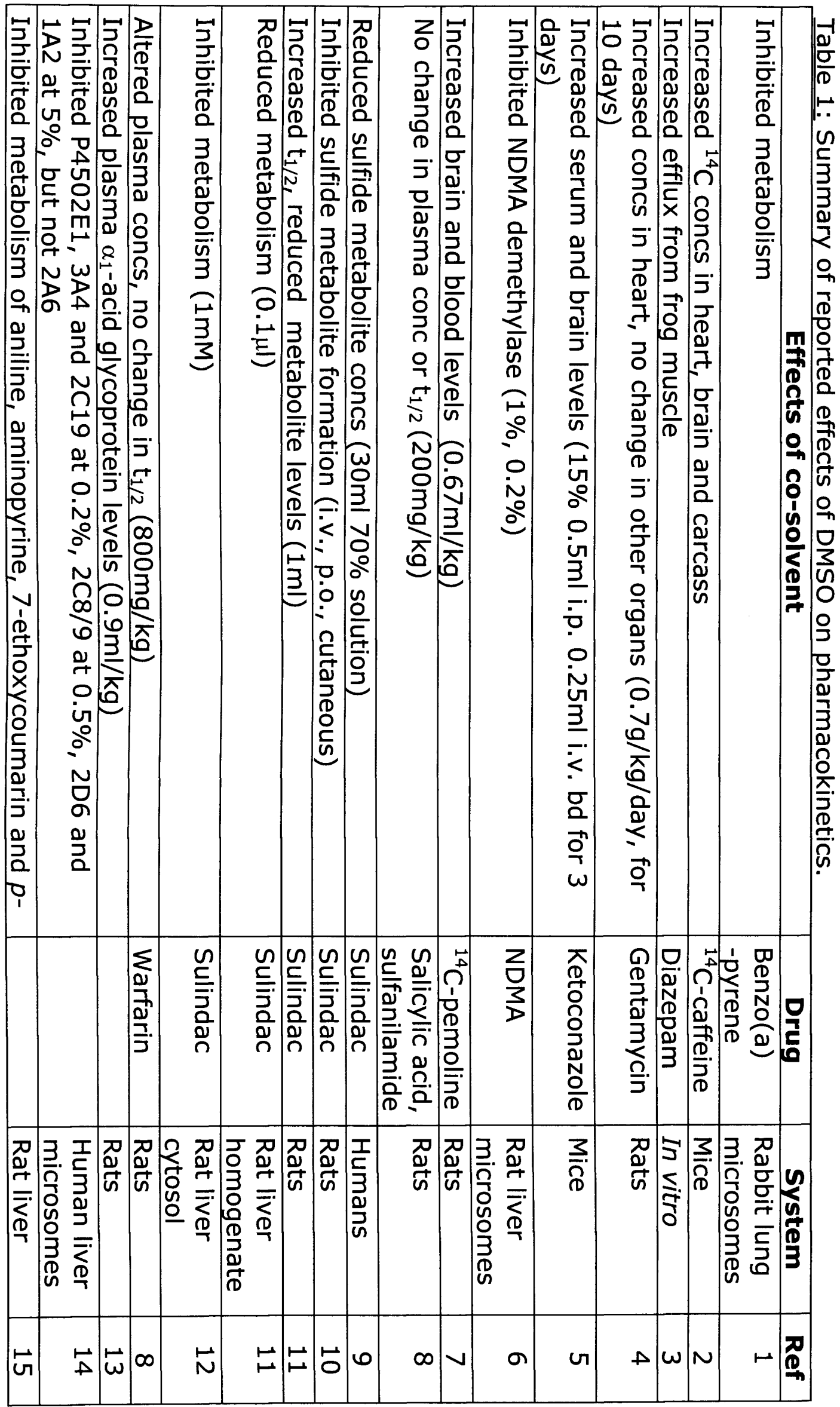




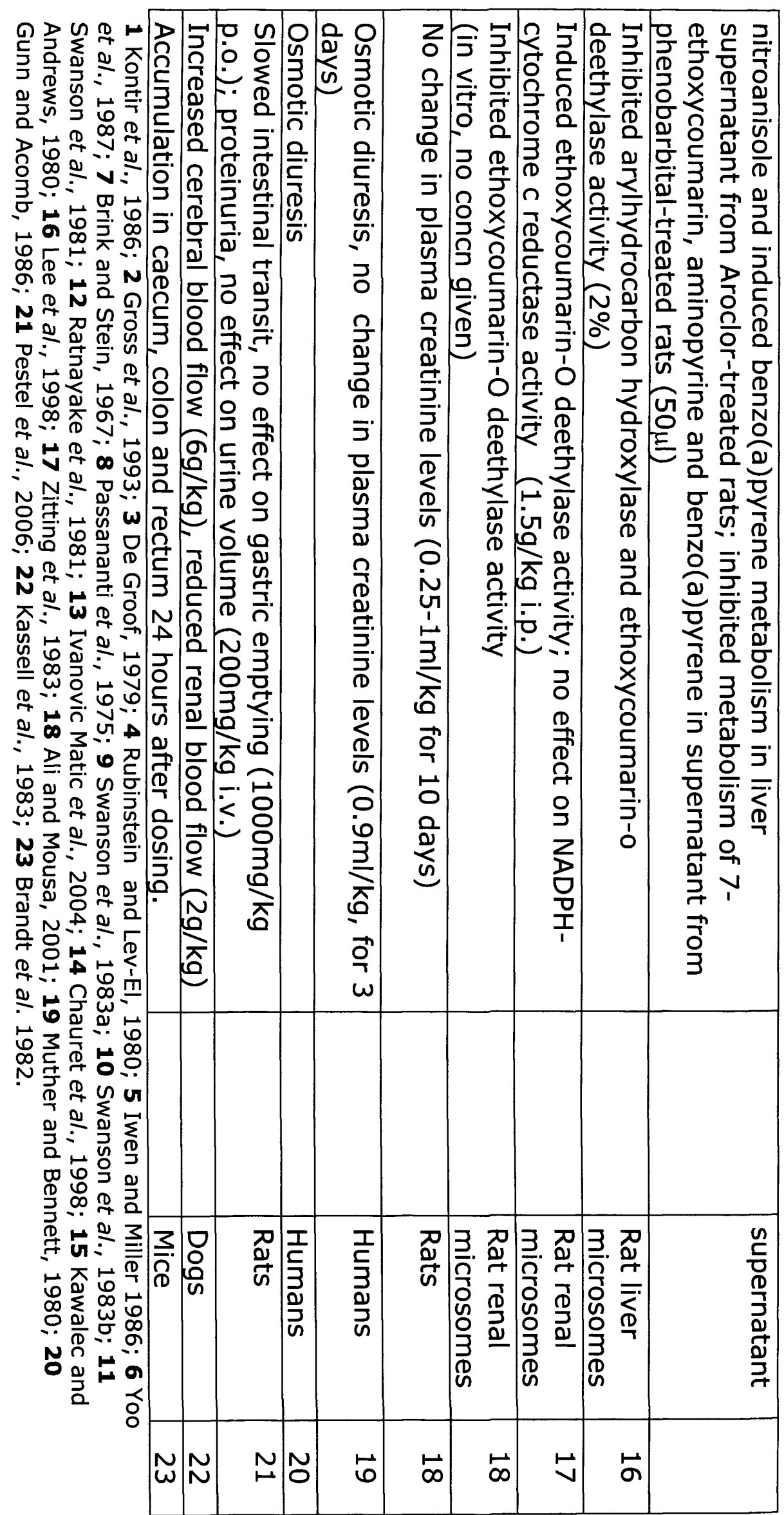




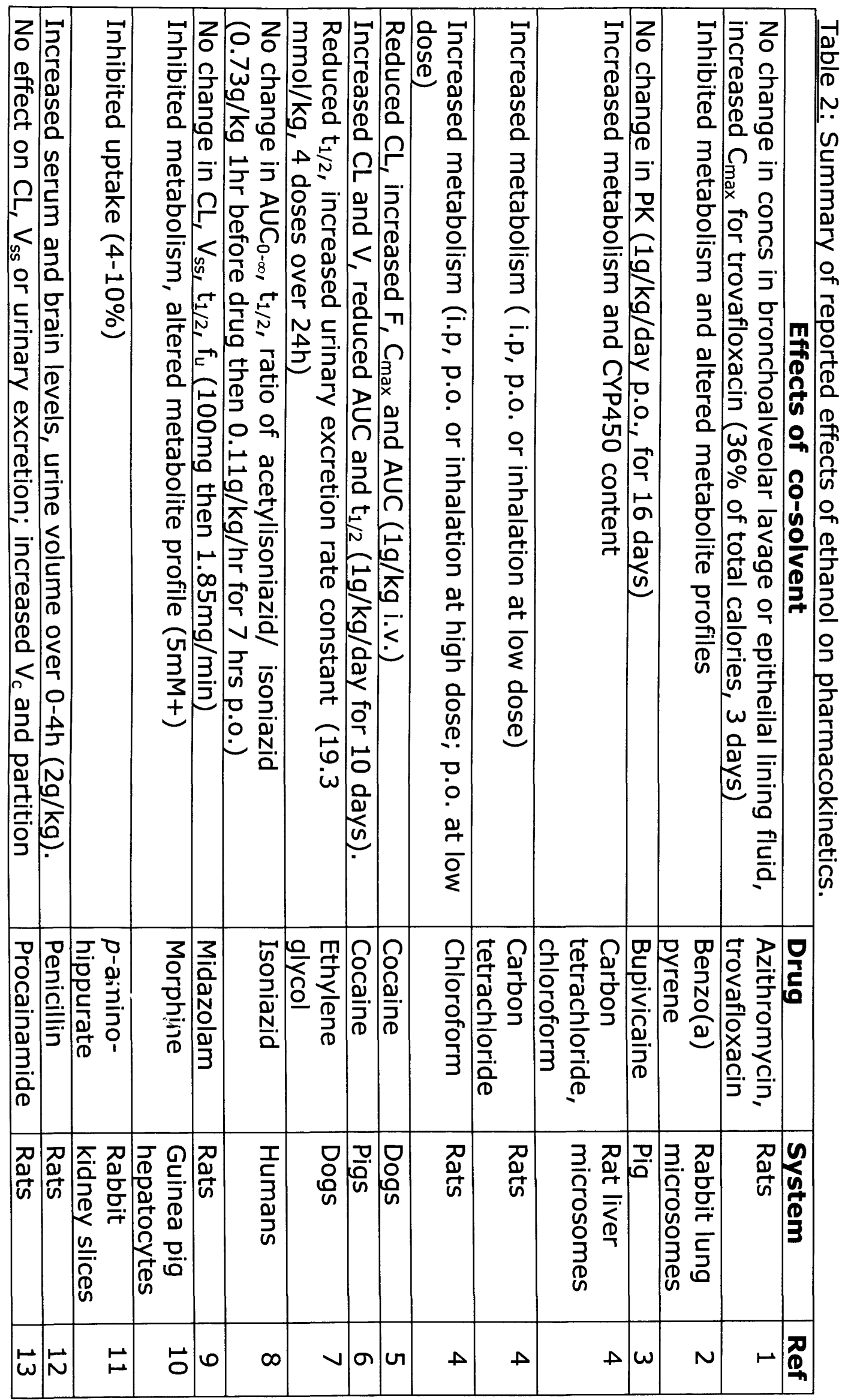




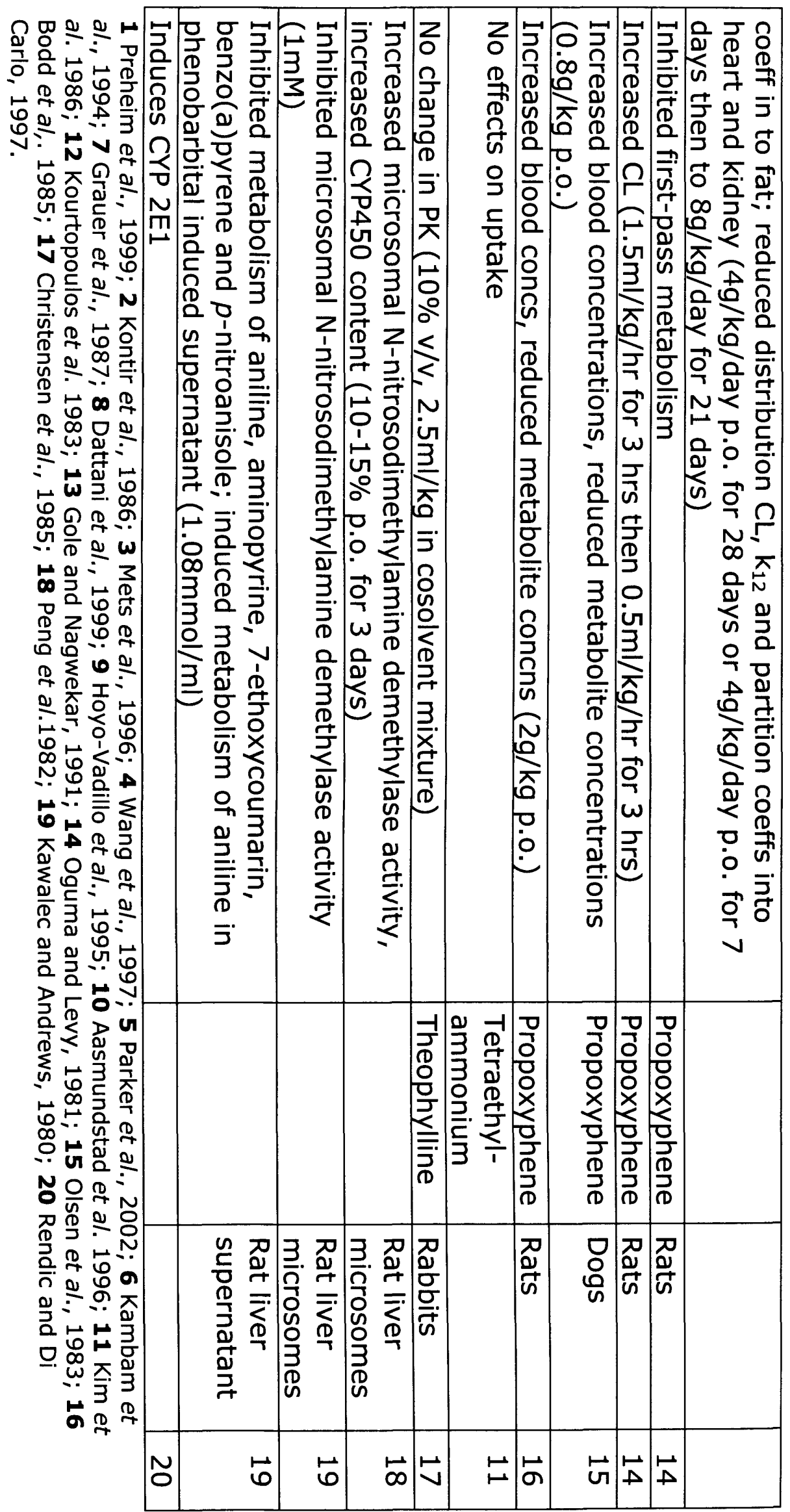




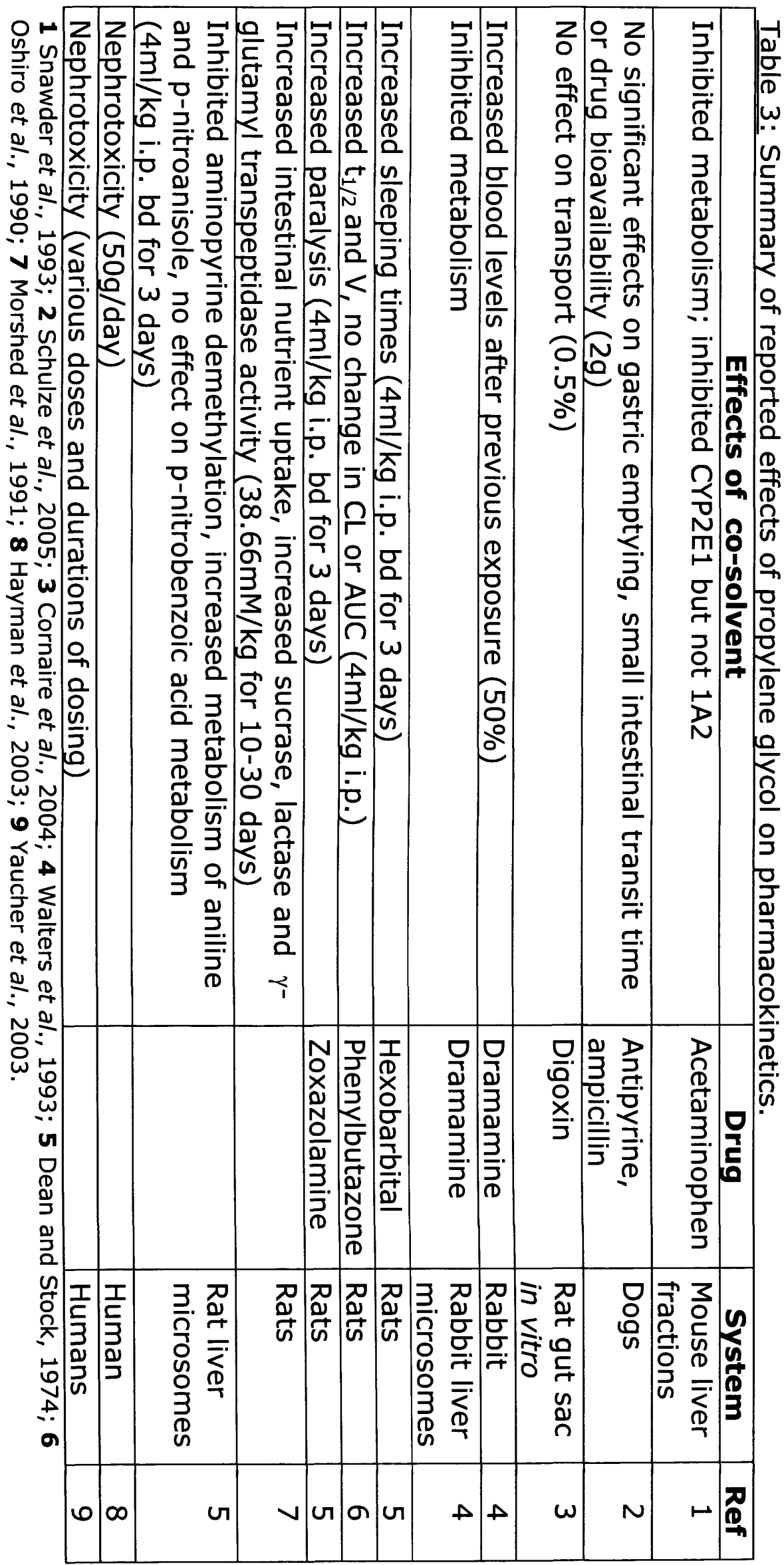




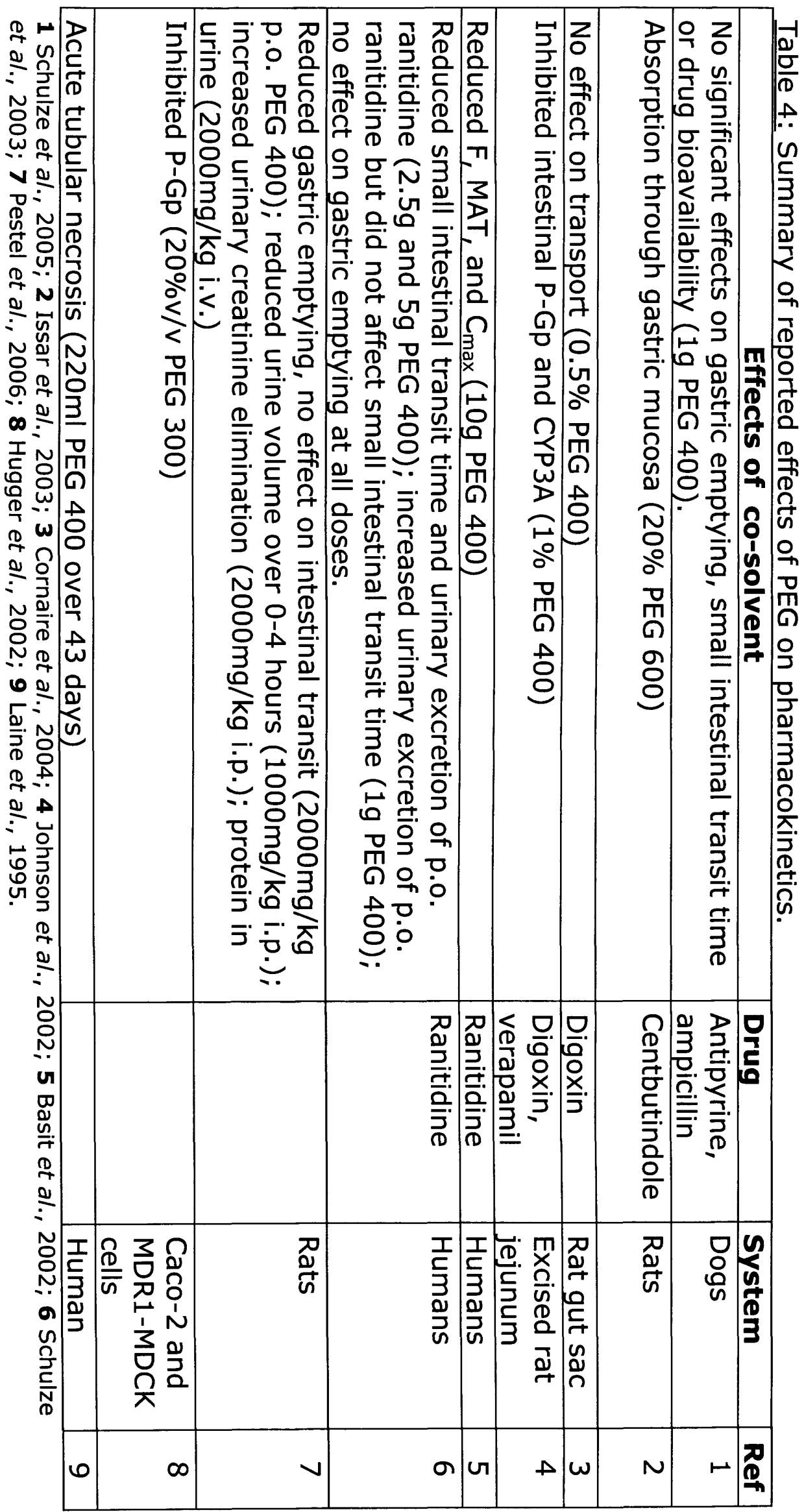



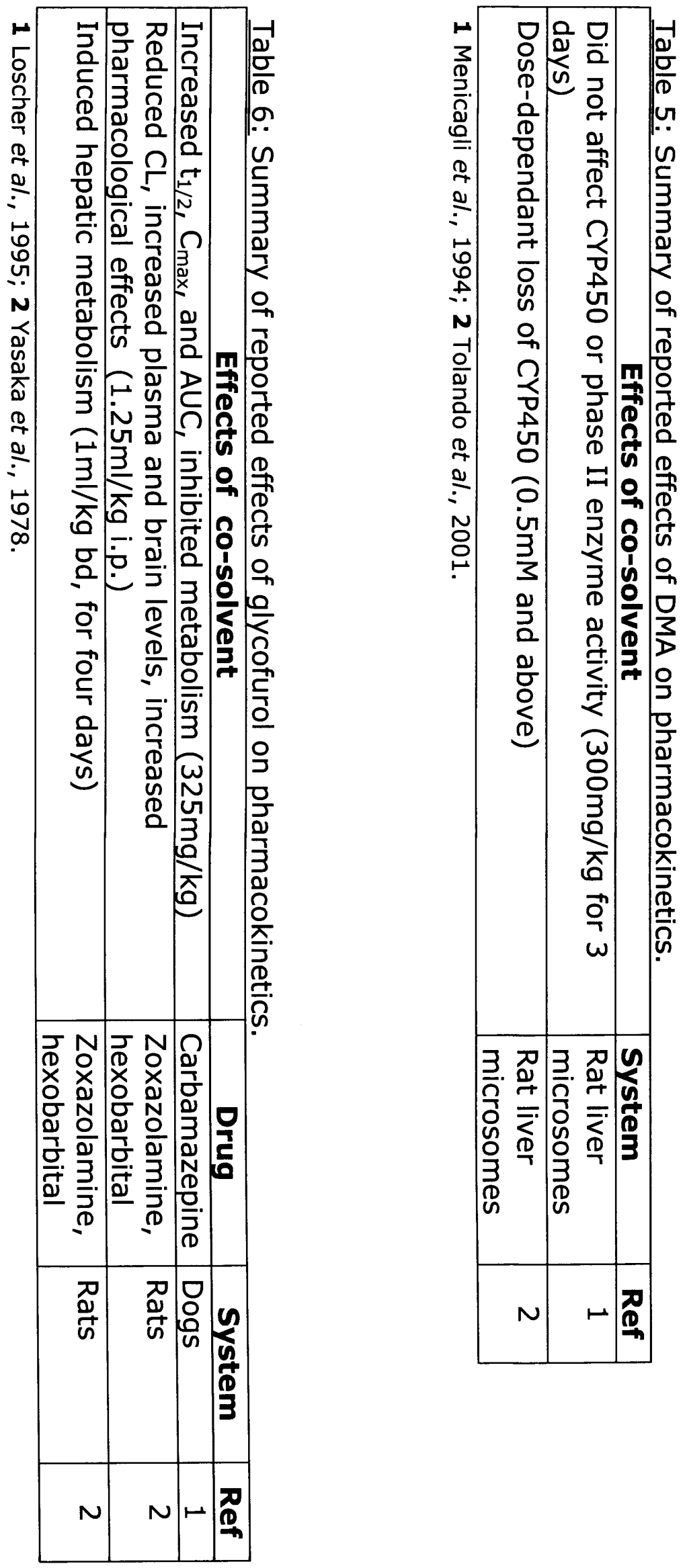


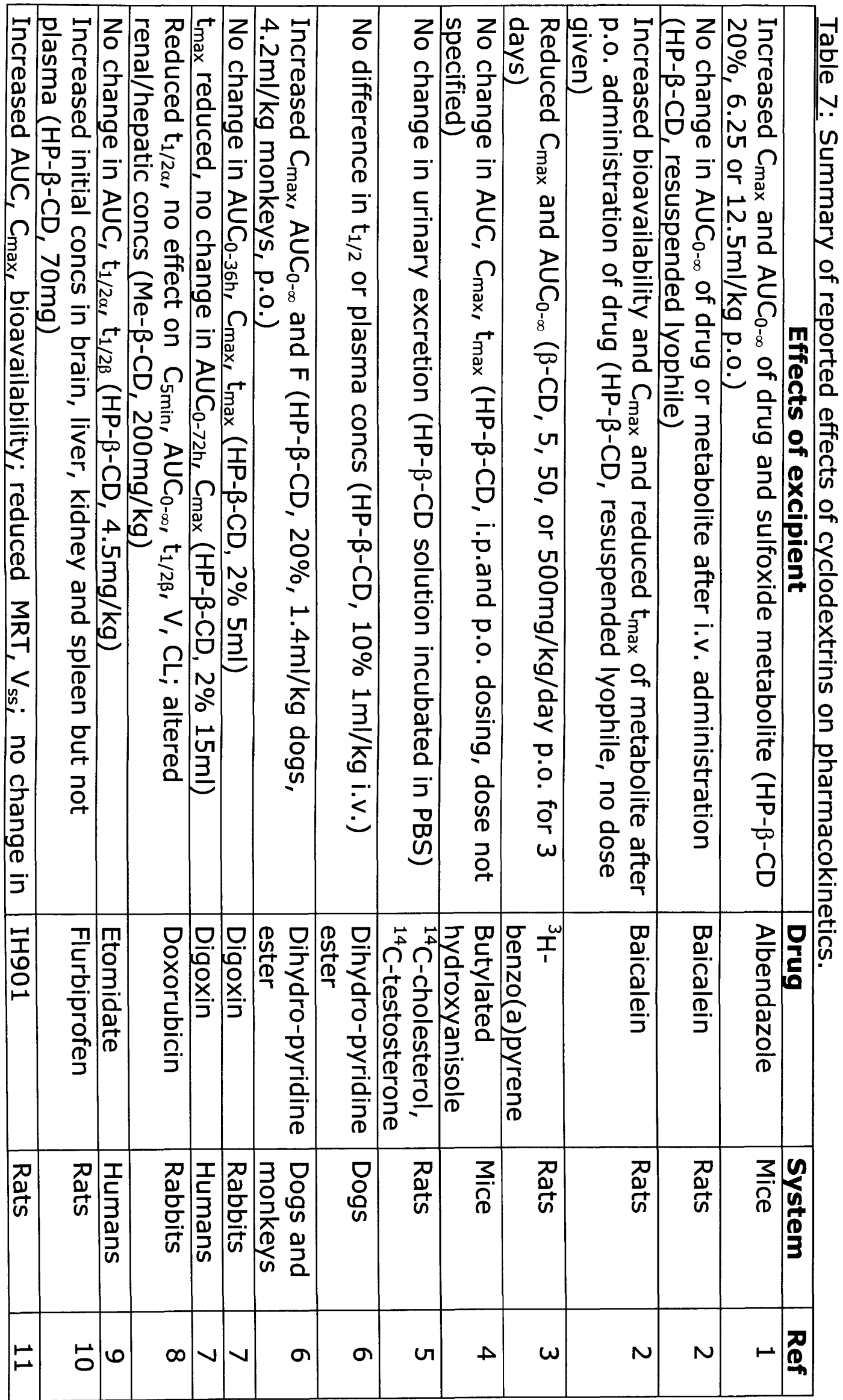


구일 の

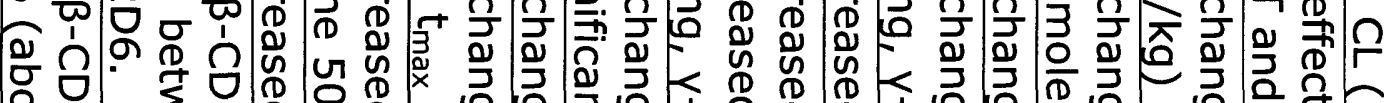
证

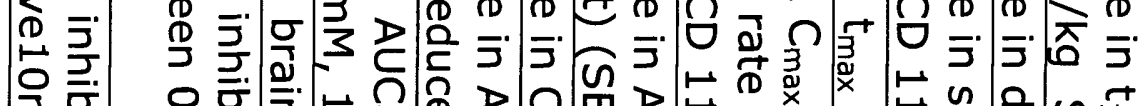

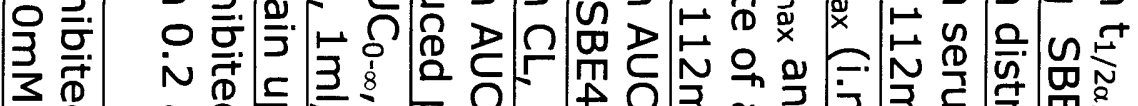

३荠 N

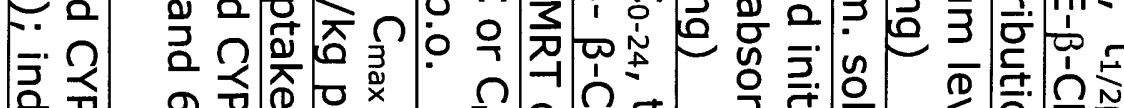

든

吕

了 w

ภำ

음 豆:

ำ ำ

古证

ชิ

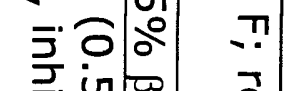

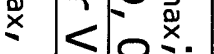

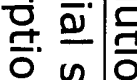

市高约商

亏.

吕

气

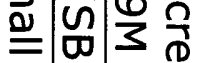

$\sum_{10}^{0}$

\$

ณ 30

들

亏.

กุ่

옹

(2)

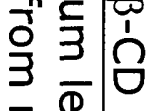

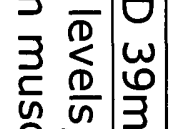

岕

党

원

궁

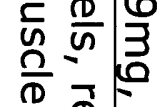

少 $\frac{3}{3}$

穴 금

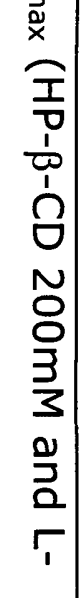

$\overline{3}$

号

용

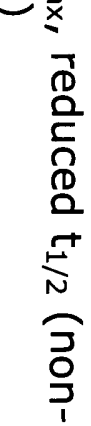

วัำ

$\frac{D}{n}-$

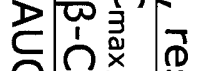

$\infty \bar{\jmath}$

30

当

盯 品

宁

䯧

号

$\therefore$ 잉

ํํำ

趋

号

윽

占号

\begin{tabular}{|c|c|c|c|c|c|c|c|c|c|c|c|c|c|}
\hline & & 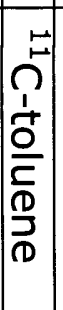 & $\begin{array}{l}\not 0 \\
0 \\
1 \\
\infty \\
1 \\
\text { م్ } \\
\text { जు }\end{array}$ & 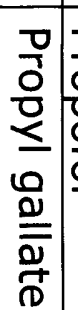 & 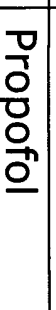 & 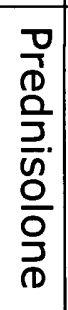 & $\begin{array}{l}\frac{0}{1} \\
\frac{0}{2} \\
\frac{\partial}{n} . \\
\frac{0}{0} \\
\frac{\partial}{0}\end{array}$ & 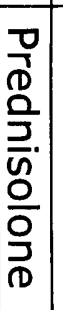 & 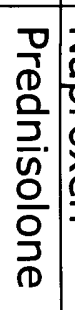 & 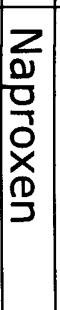 & 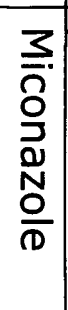 & 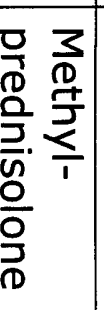 & 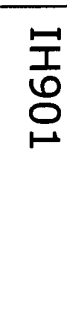 \\
\hline $\begin{array}{l}5 \\
\vdots \\
5 \\
\delta\end{array}$ & $\begin{array}{l}5 \\
5 \\
5 \\
5\end{array}$ & 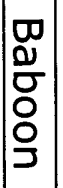 & $\begin{array}{l}\frac{n}{7} \\
\mathbb{\Phi} \\
\frac{\mathbb{D}}{0}\end{array}$ & $\frac{3}{\bar{D}}$ & $\frac{0}{n}$ & $\begin{array}{l}\not{D} \\
\frac{\sigma}{\sigma} \\
\frac{\sigma}{\vec{\sigma}} \\
\text { (1) }\end{array}$ & 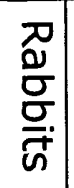 & $\begin{array}{l}\underline{0} \\
\underline{\sigma} \\
\sigma \\
\frac{\sigma}{\sigma} \\
\vec{\sigma}\end{array}$ & $\begin{array}{l}\overline{0} \\
\bar{\sigma} \\
\frac{\sigma}{\sigma} \\
\dot{v}\end{array}$ & $\mid \begin{array}{l}\not{D} \\
\stackrel{\sim}{\sim} \\
\tilde{\omega}\end{array}$ & $\begin{array}{l}\frac{n}{7} \\
\mathbb{D} \\
\mathbb{D} \\
\mathbb{D}\end{array}$ & 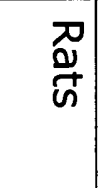 & $\begin{array}{l}\text { Do } \\
\text { W } \\
\text { un }\end{array}$ \\
\hline 깅 & 이 & $\vec{\omega}$ & $\vec{\infty}$ & 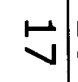 & $\ddot{\sigma}$ & $\vec{v}$ & $\vec{\Delta}$ & $\vec{\Delta}$ & $\vec{\Delta}$ & 10 & $\vec{\omega}$ & $\stackrel{\sim}{N}$ & $\mapsto$ \\
\hline
\end{tabular}




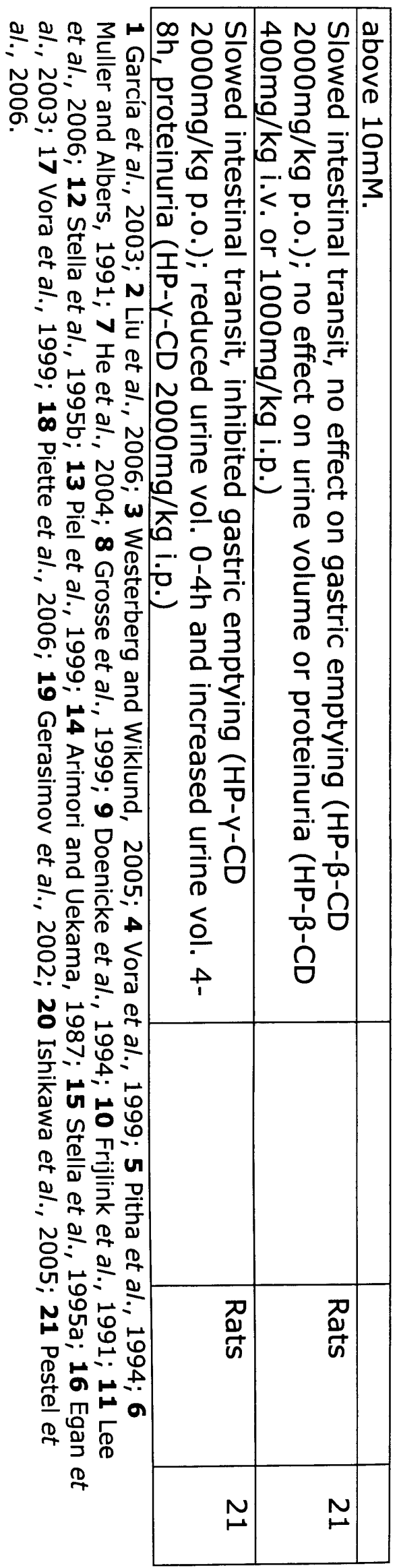




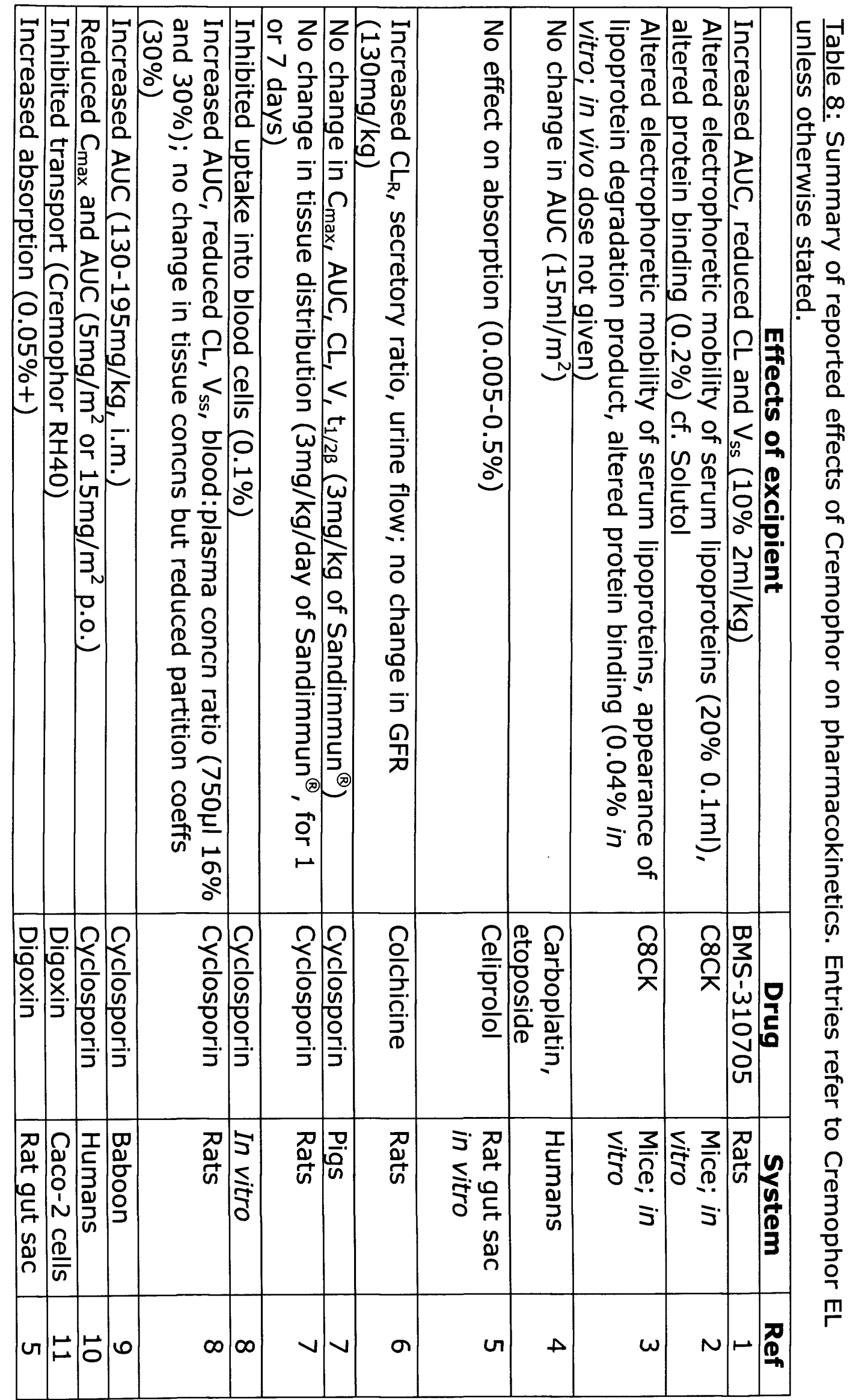




\begin{tabular}{|c|c|c|c|c|c|c|c|c|c|c|c|c|c|}
\hline 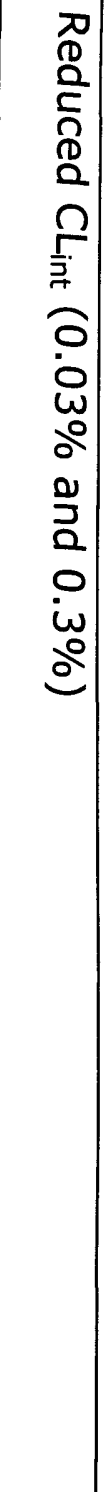 & 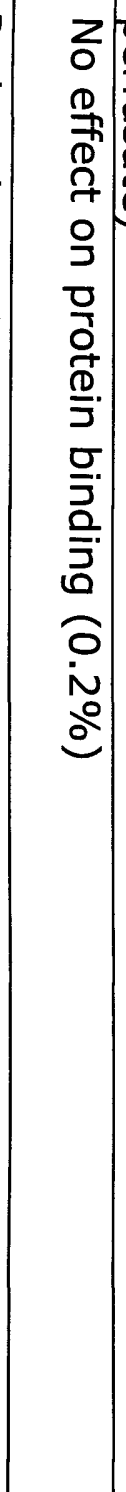 & 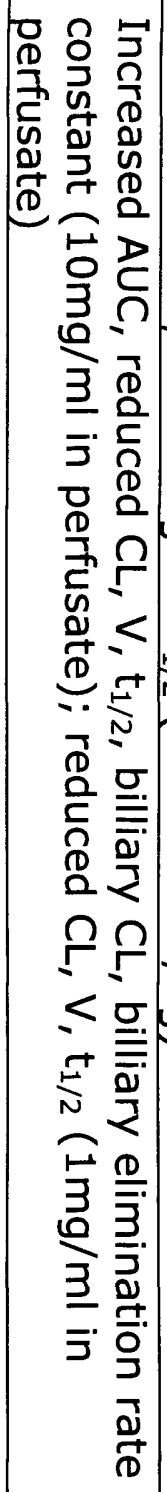 & 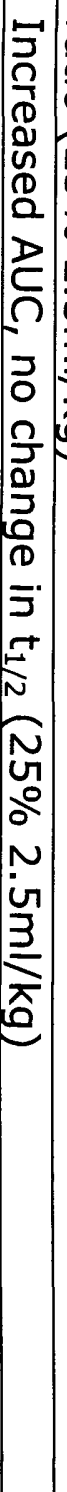 & 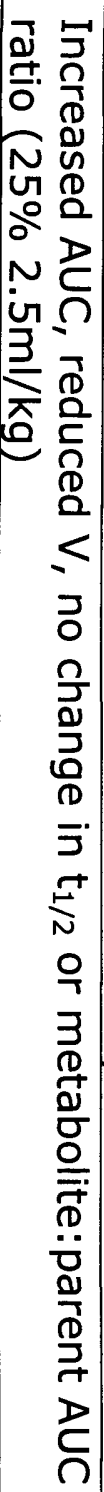 & 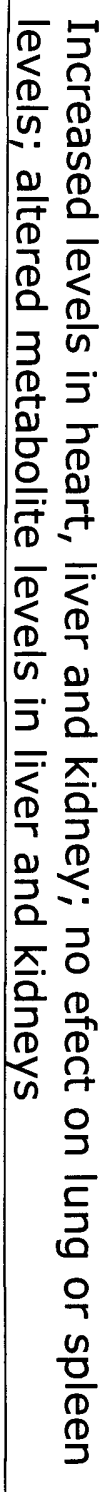 & 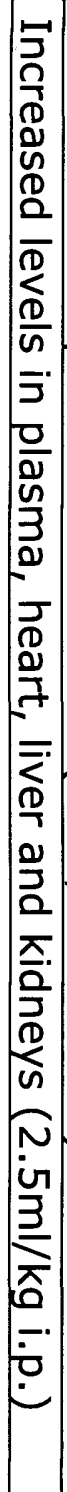 & 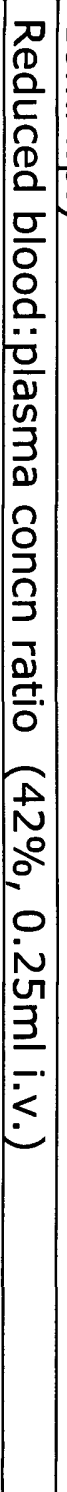 & 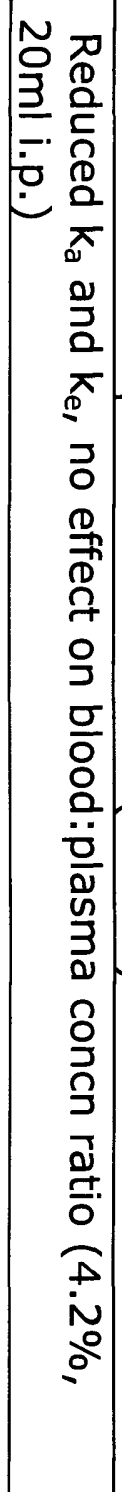 & 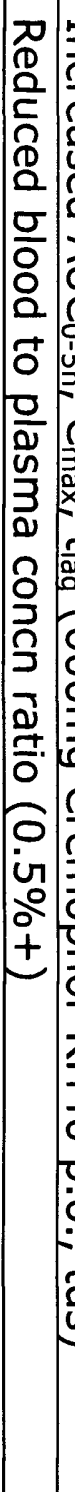 & 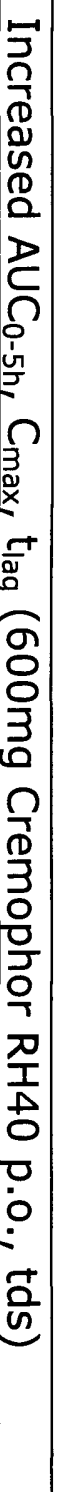 & 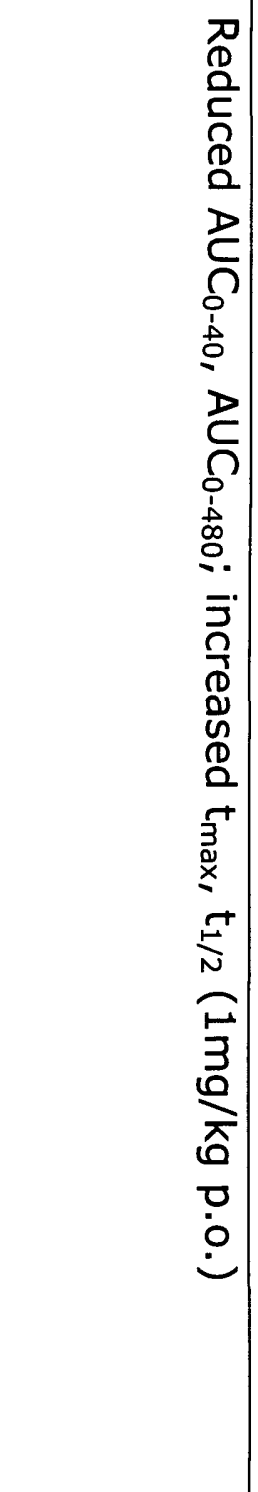 & 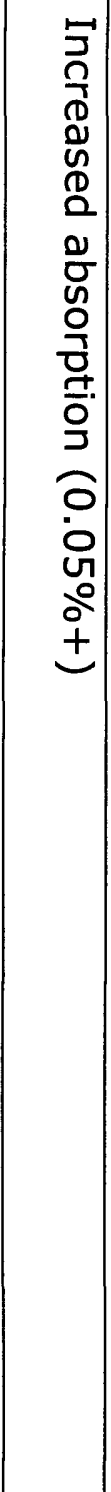 & \\
\hline $\begin{array}{l}3 \\
\frac{3}{2} \\
N \\
\frac{0}{2} \\
3\end{array}$ & 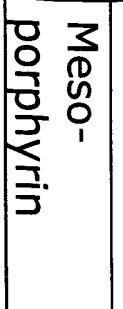 & $\begin{array}{l}\frac{\pi}{+} \\
\frac{0}{0} \\
\frac{0}{D} \\
\frac{0}{D}\end{array}$ & 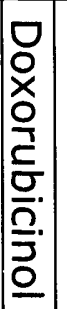 & 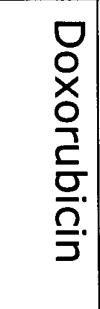 & 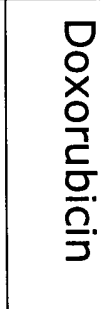 & 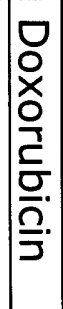 & 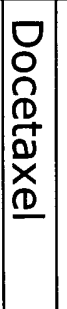 & 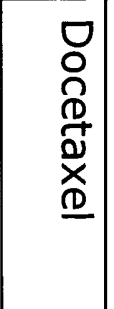 & 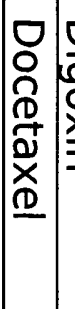 & 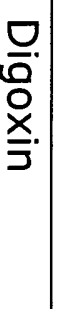 & 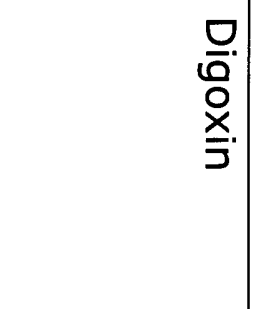 & $\begin{array}{l}\text { 뭄 } \\
\text { 을. } \\
\text { ․ }\end{array}$ & \\
\hline 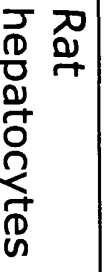 & $\begin{array}{l}5 \\
5 \\
\vdots \\
\vdots \\
\vdots\end{array}$ & 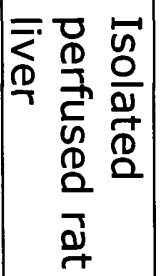 & & $\frac{3}{\grave{D}}$ & $\frac{3}{\bar{n}}$ & $\frac{3}{\bar{\alpha}}$ & $\begin{array}{l}\not{D} \\
\stackrel{\sim}{\sim} \\
\tilde{G}\end{array}$ & $\begin{array}{l}\text { Do } \\
\stackrel{\sim}{+} \\
\vec{v}\end{array}$ & $\mid \begin{array}{l}5 \\
\overline{5} \\
\bar{\sigma}\end{array}$ & 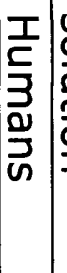 & 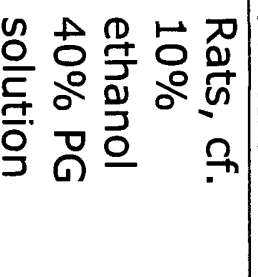 & 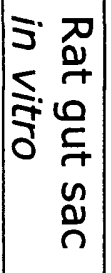 & $\begin{array}{l}5 \\
\vdots \\
\vdots \\
\vdots\end{array}$ \\
\hline $\overrightarrow{0}$ & $N$ & $\vec{\infty}$ & $\stackrel{v}{v}$ & V & ڤே & $\vec{v}$ & $\vec{A}$ & $\vec{\Delta}$ & $\vec{\Delta}$ & $\mid \vec{\omega}$ & 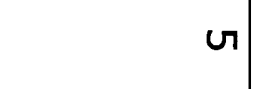 & $\stackrel{\sim}{N}$ & \\
\hline
\end{tabular}




\begin{tabular}{|c|c|c|c|c|c|c|c|c|c|c|c|c|c|c|c|}
\hline 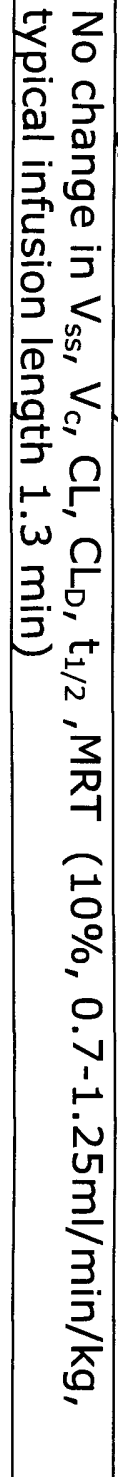 & 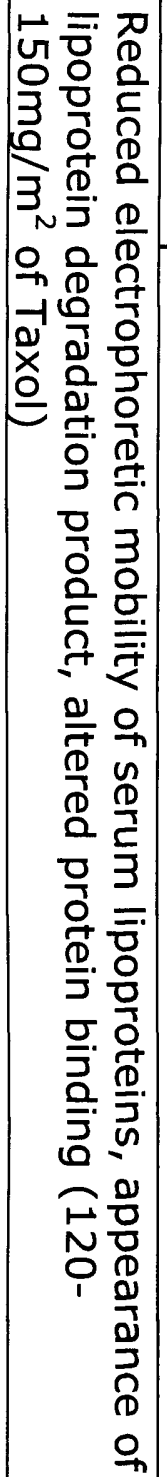 & 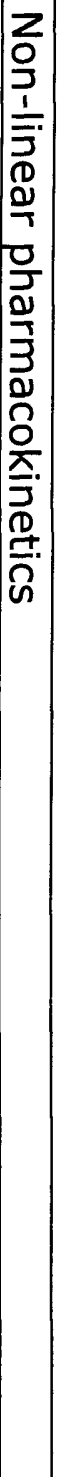 & 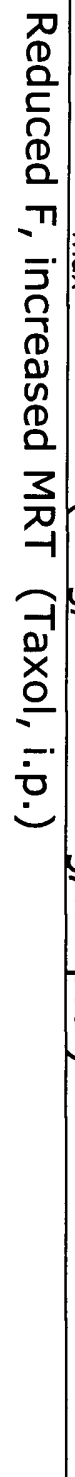 & 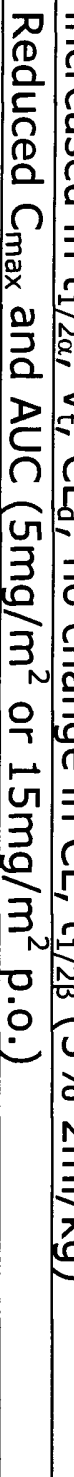 & 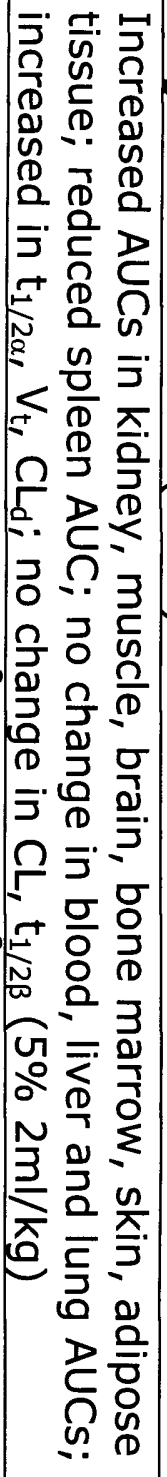 & 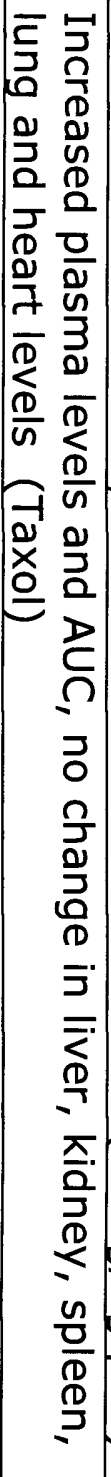 & 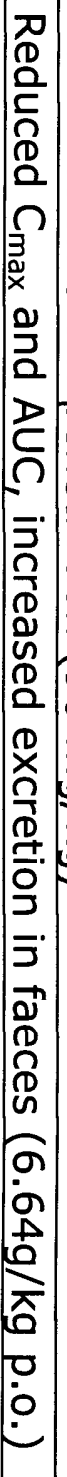 & 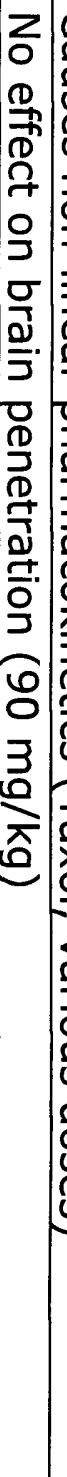 & 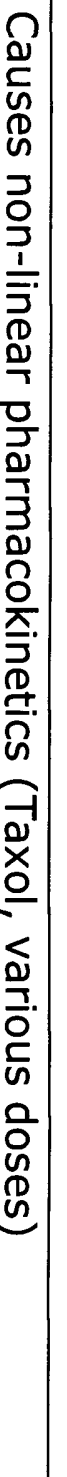 & 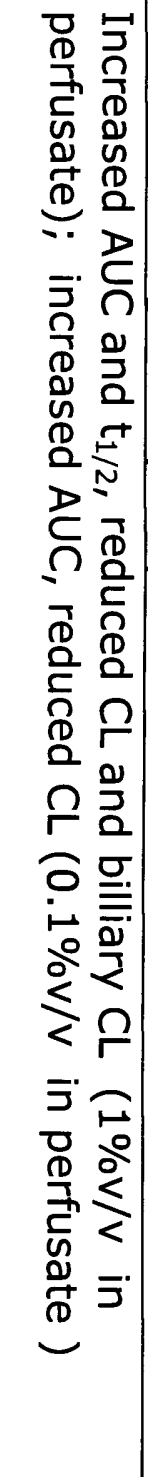 & 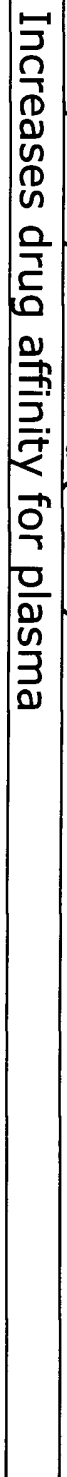 & 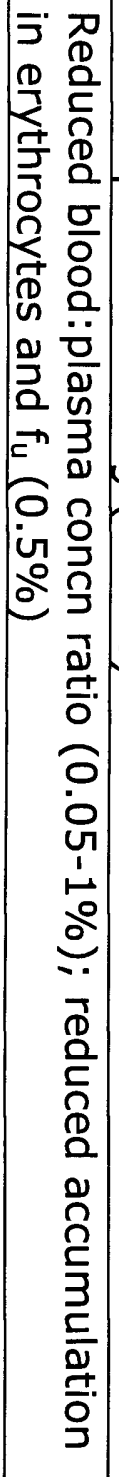 & 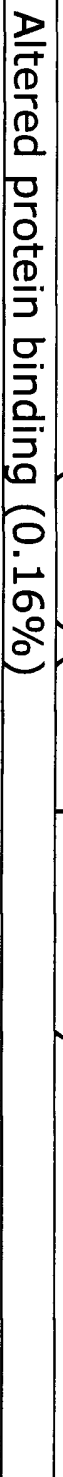 & 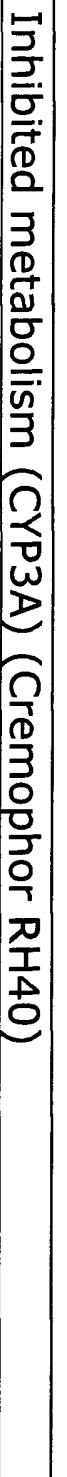 & 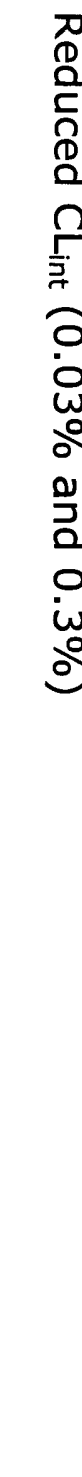 \\
\hline $\begin{array}{l}\text { 무 } \\
\text { 음 } \\
\text { 움 } \\
\text { 으 }\end{array}$ & 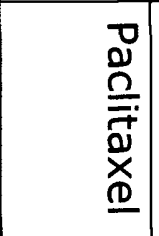 & 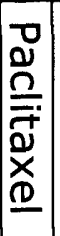 & 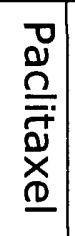 & 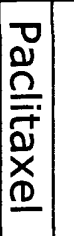 & 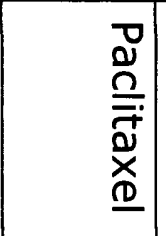 & 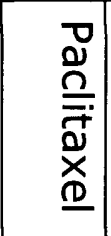 & 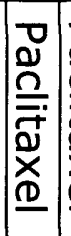 & 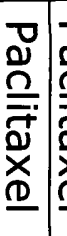 & 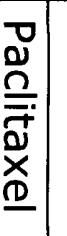 & 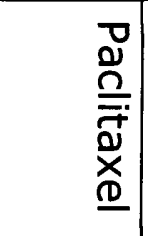 & 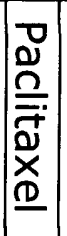 & 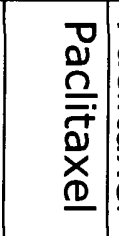 & 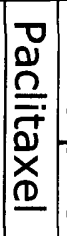 & 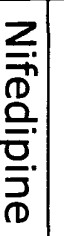 & $\begin{array}{l}3 \\
\frac{3}{0} \\
N \\
0 \\
\frac{0}{3}\end{array}$ \\
\hline $\begin{array}{l}\not{D} \\
\stackrel{\sim}{\sim} \\
\dot{\omega}\end{array}$ & $\begin{array}{l}\frac{1}{5} \\
\frac{3}{3} \\
\stackrel{2}{د} \\
\backsim\end{array}$ & 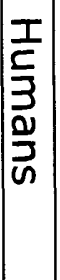 & 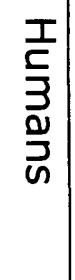 & 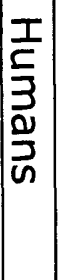 & $\begin{array}{l}\mathbb{D} \\
\stackrel{d}{\omega} \\
\vec{v}\end{array}$ & $\frac{3}{\bar{D}}$ & $\frac{3}{\overline{\bar{\lambda}}}$ & $\overline{\bar{n}}$ & $\overline{3}$ & 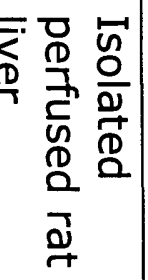 & $\begin{array}{l}5 \\
\mathbf{5} \\
\mathbf{5} \\
\mathbf{5}\end{array}$ & $\begin{array}{l}5 \\
\vdots \\
\vdots \\
\end{array}$ & $\left|\begin{array}{c}- \\
\vdots \\
\vdots \\
\vdots \\
\vdots \\
\vdots\end{array}\right|$ & 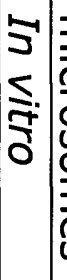 & 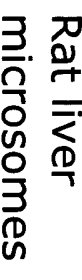 \\
\hline$\underset{N}{\omega}$ & $\underset{\omega}{\omega}$ & $\underline{\omega}$ & $\underline{\omega}$ & 10 & 6 & $\stackrel{\infty}{\infty}$ & $\stackrel{N}{V}$ & a & v & $\stackrel{N}{+}$ & $\tilde{\omega}$ & $N$ & $\stackrel{N}{\sim}$ & '̊ & 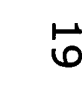 \\
\hline
\end{tabular}




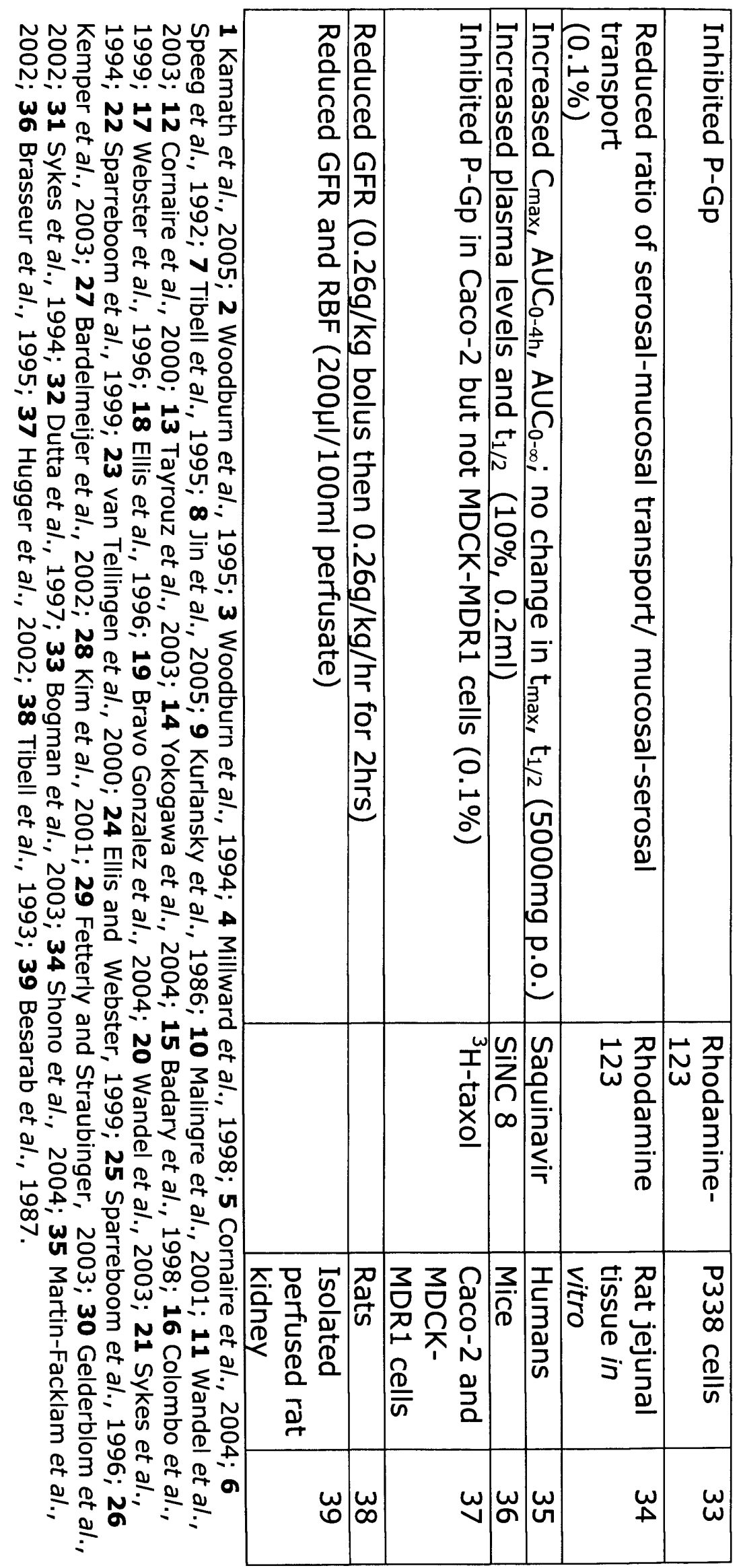




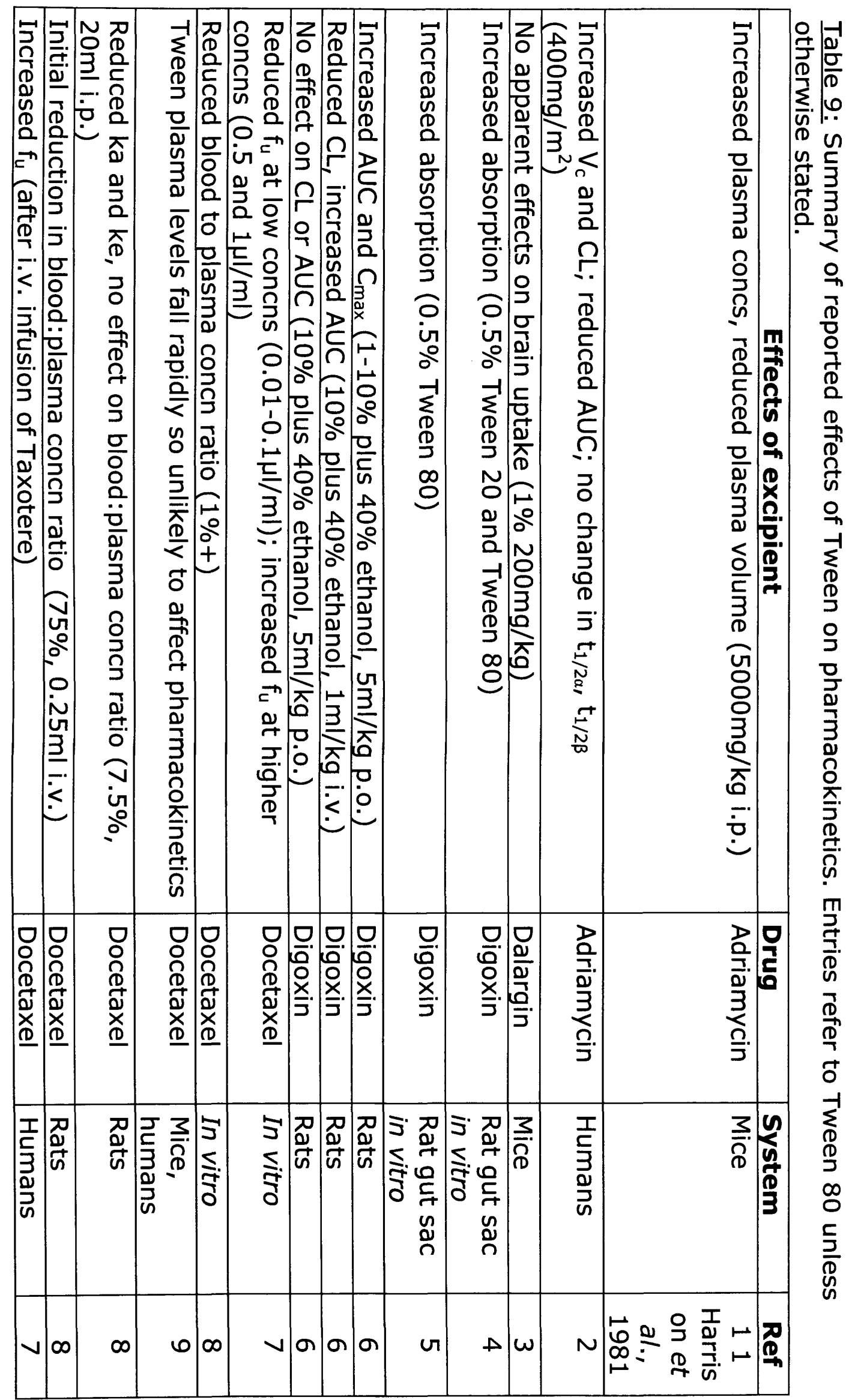




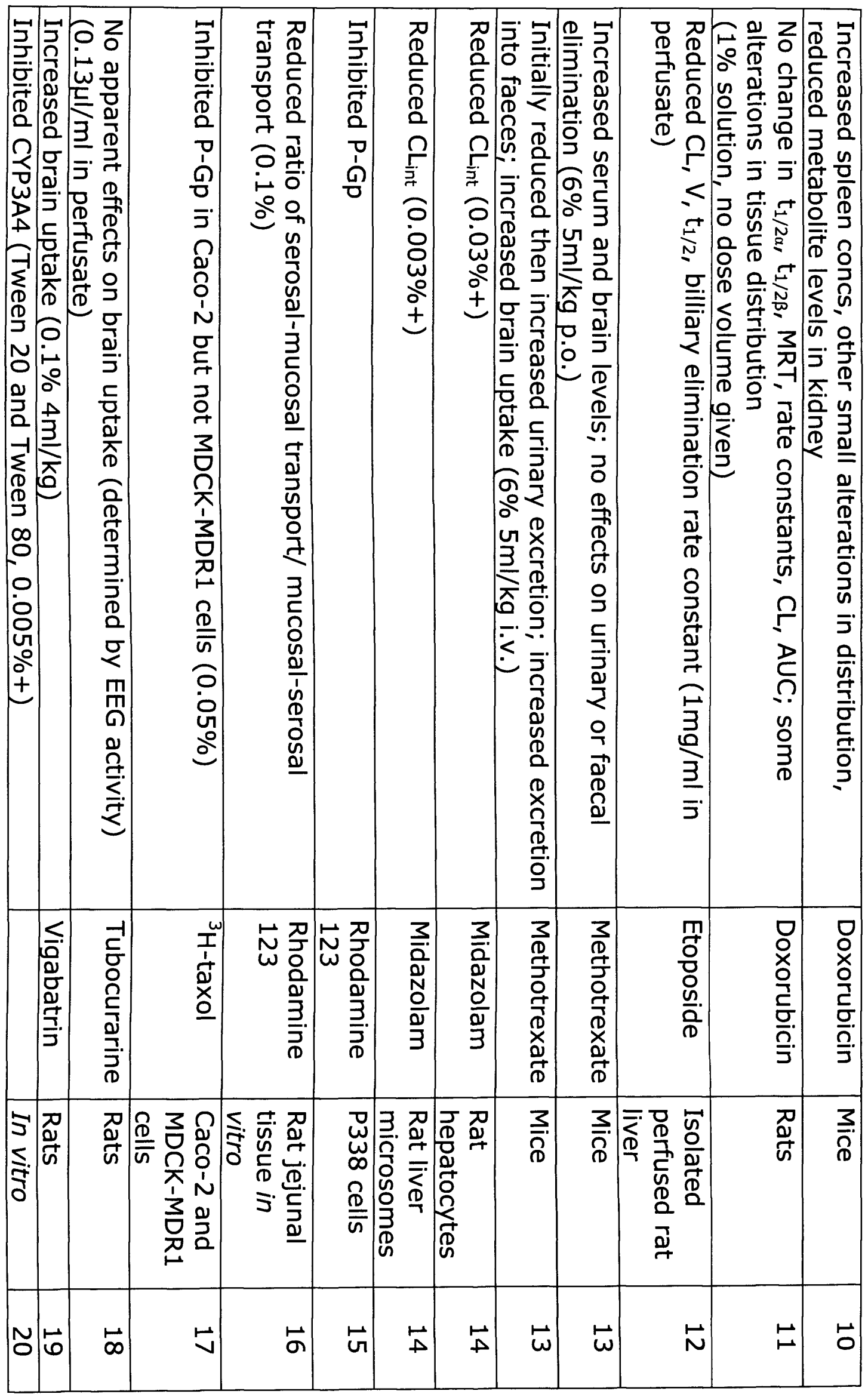




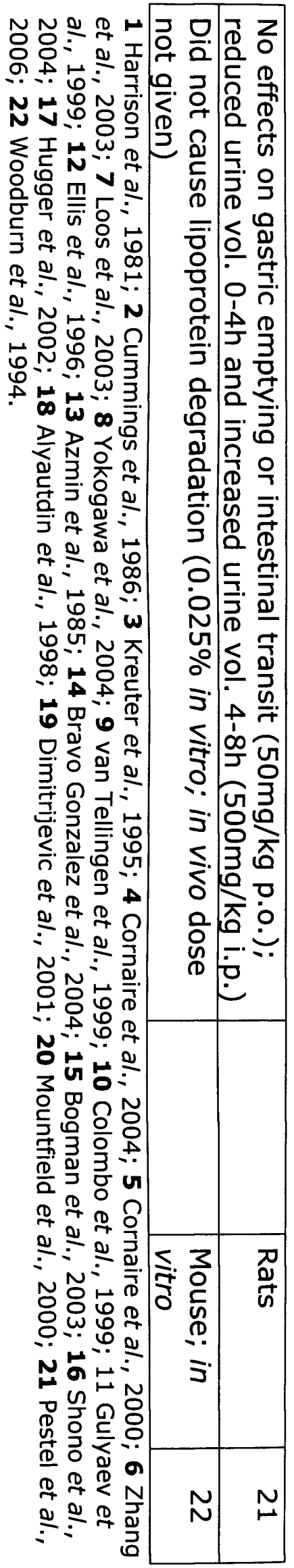




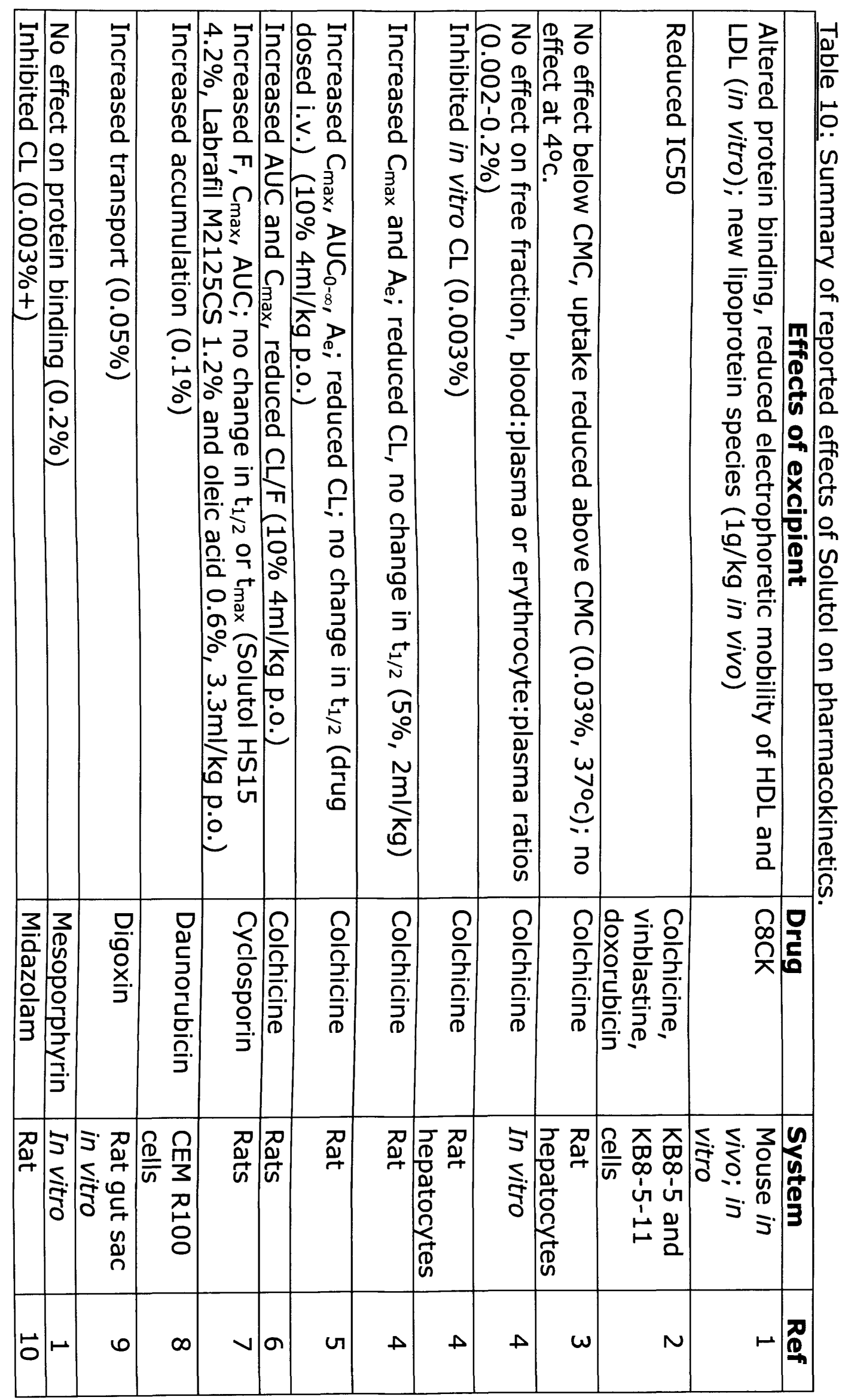




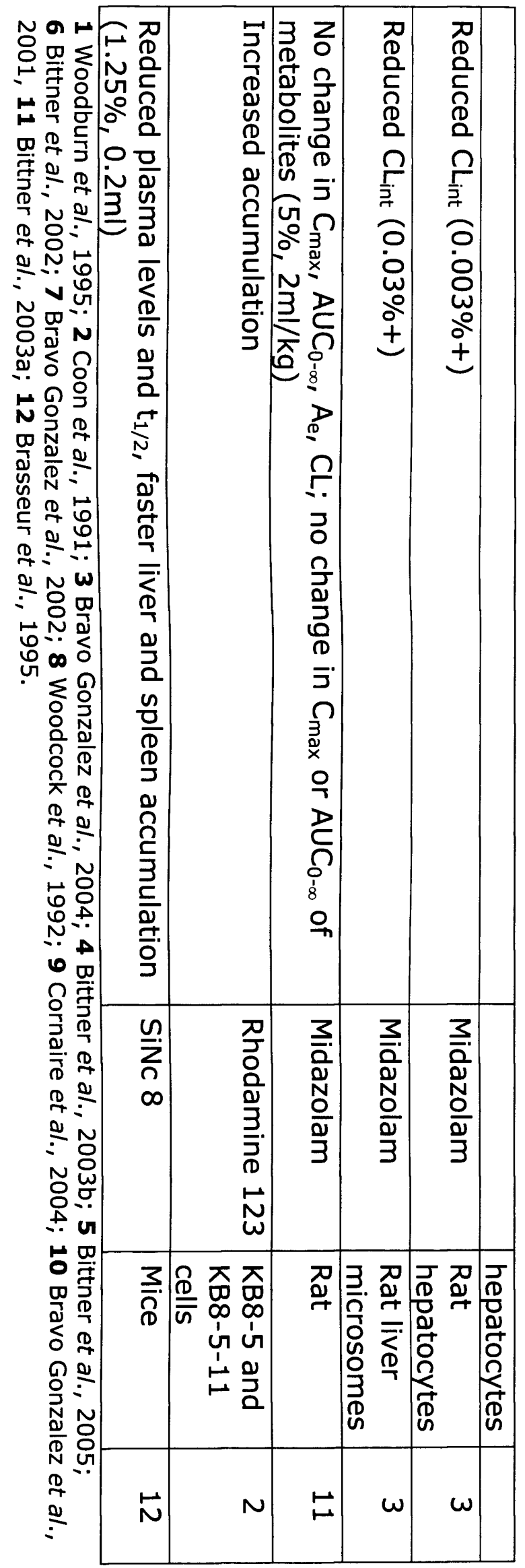




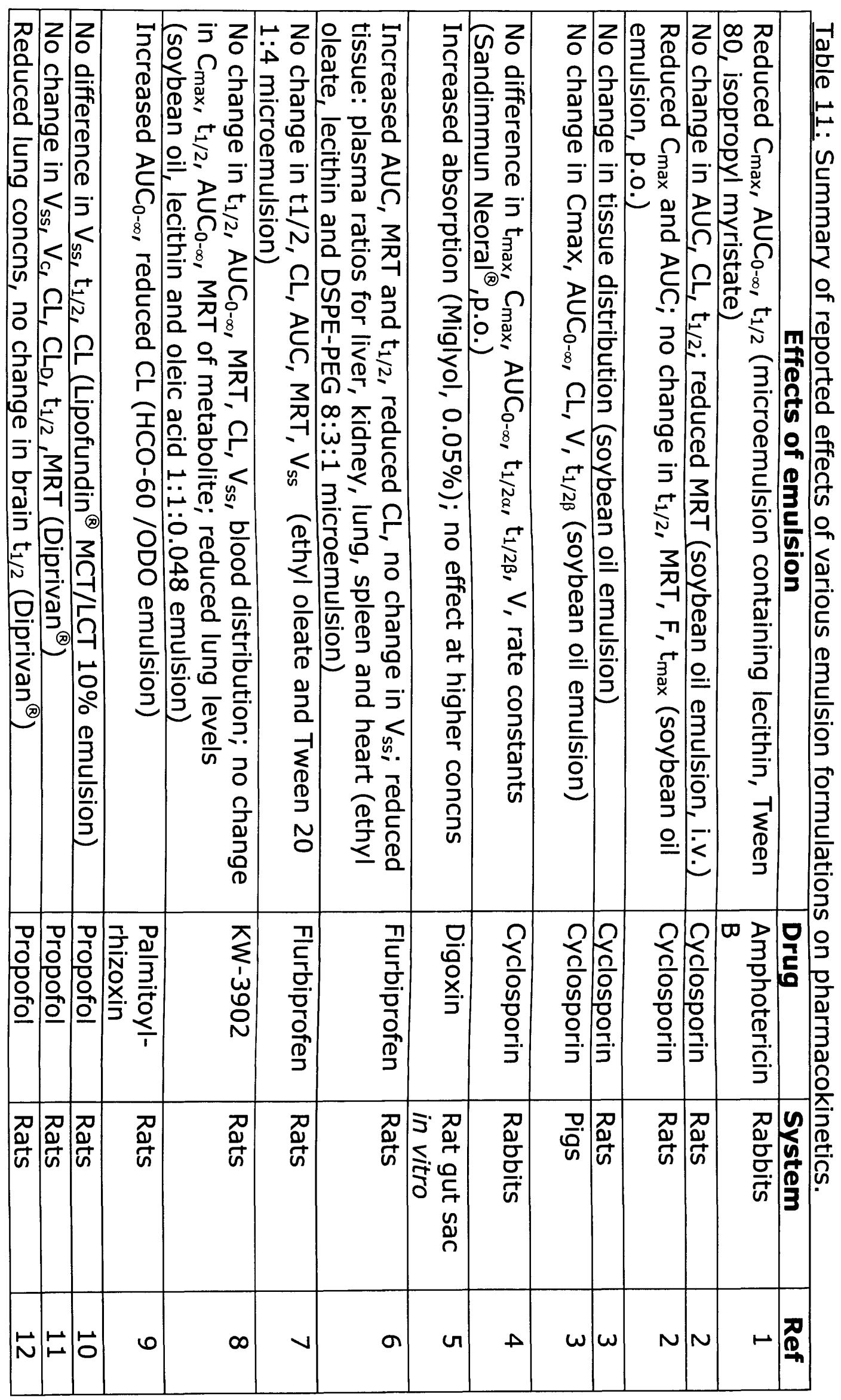




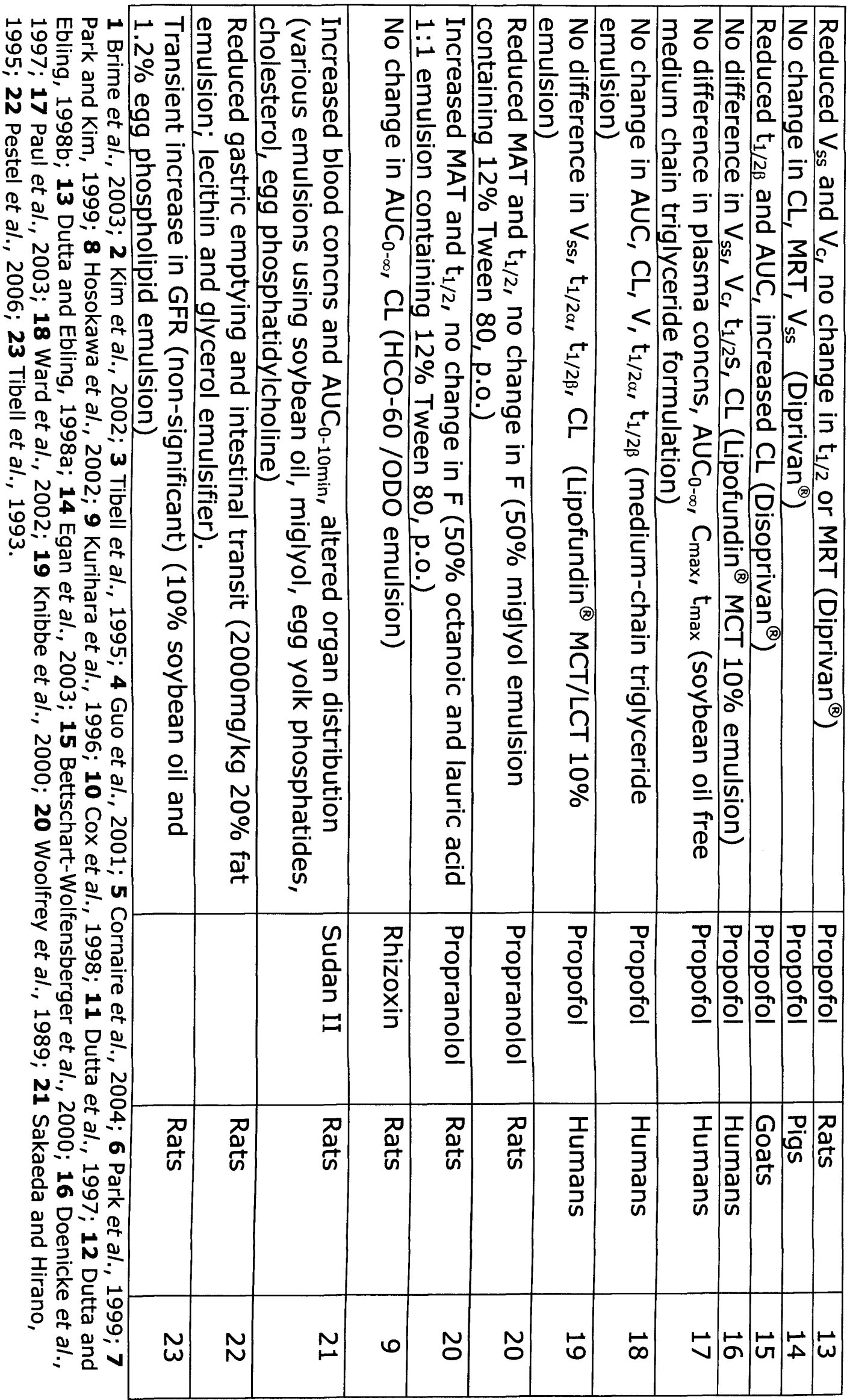




\section{Appendix 2: Structures of compounds used}<smiles>Cc1c(N(C)C)c(=O)n(-c2ccccc2)n1C</smiles>

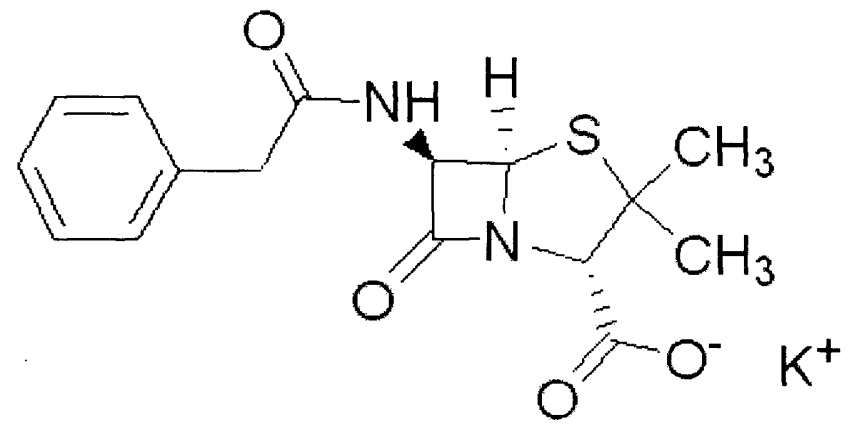<smiles>Cn1c(=O)c2c(ncn2C)n(C)c1=O</smiles>

Benzylpenicilin potassium salt<smiles>CNC(=NCCSCc1nc[nH]c1C)NC#N</smiles><smiles>O=C(O)CN(CCN(CC(=O)O)CC(=O)O)CC(=O)O</smiles>

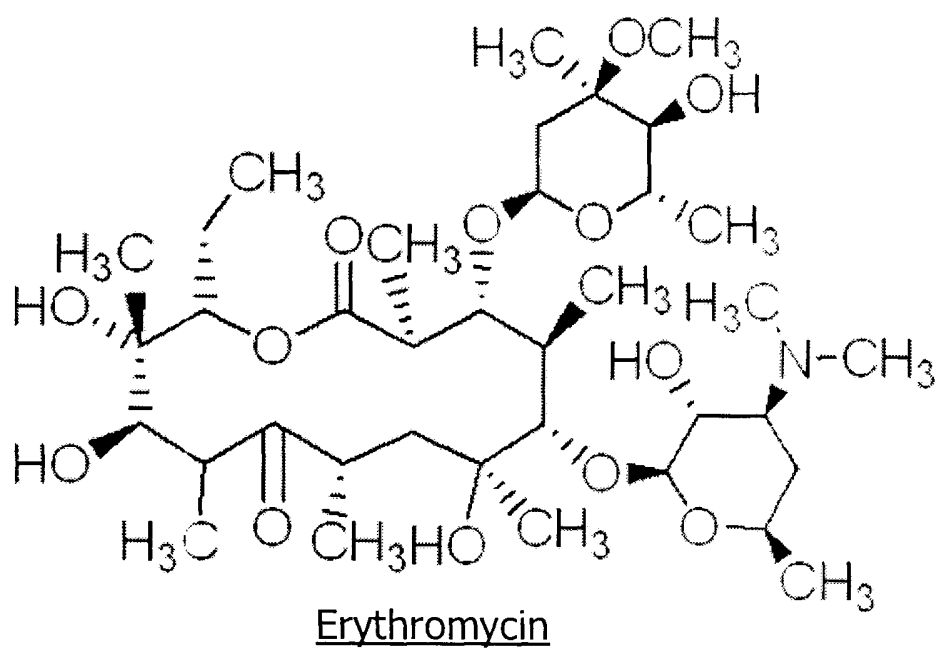<smiles>Cc1cccc(C)c1NC(=O)CNCC(=O)O</smiles> 


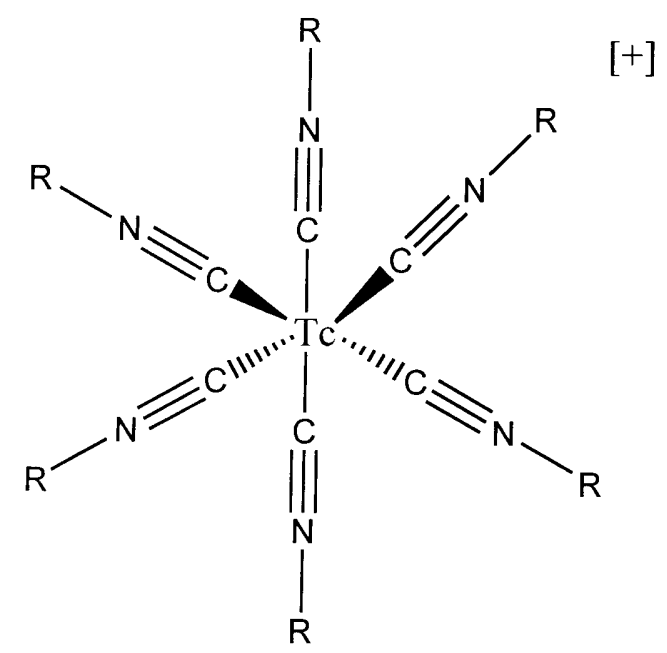

$\left(\mathrm{R}=\mathrm{CH}_{2} \mathrm{C9m} \mathrm{Tc}\left(\mathrm{CH}_{3}\right)_{2} \mathrm{OCH}_{3}\right)$
$\mathrm{CH}_{3}$<smiles>CN(C)N=O</smiles>

NDMA

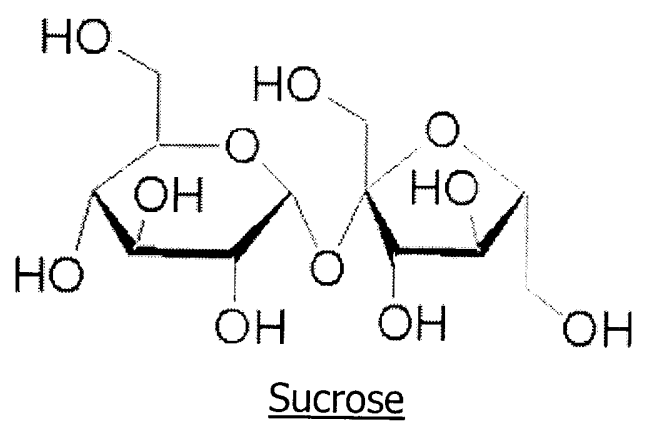

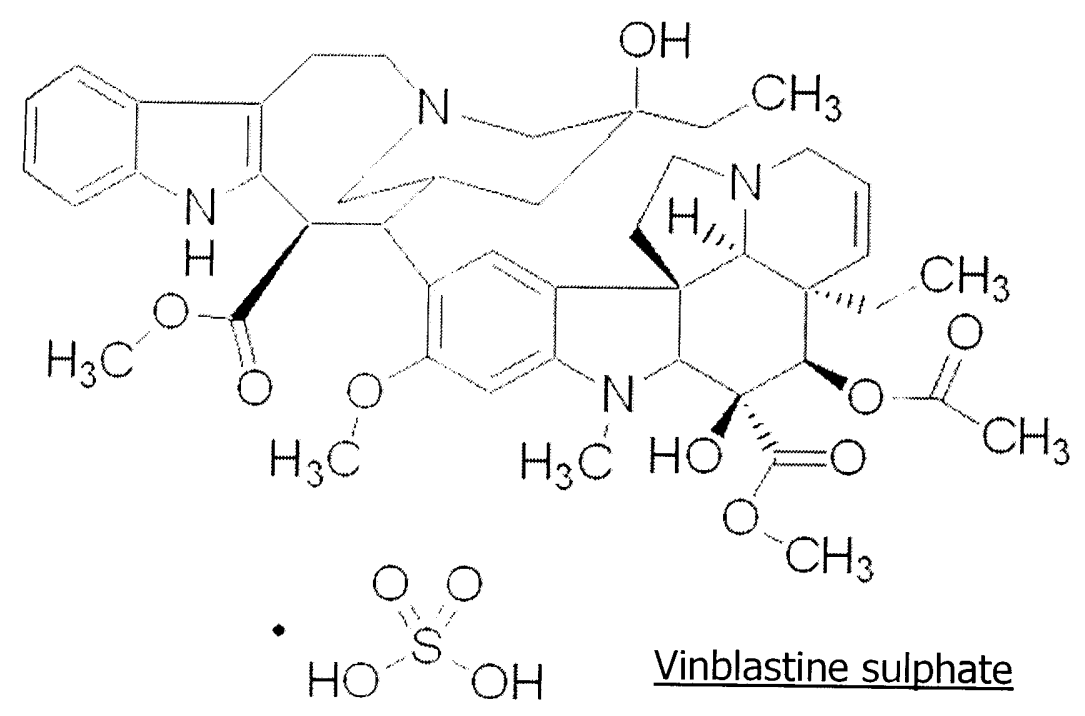

